\title{
Interdisziplinärer Kongress | Ultraschall 2018
}

42. Dreiländertreffen SGUM | DEGUM | ÖGUM

Datum/Ort:

14.-16. November 2018, Congress Center, Basel
Kongresspräsidentin: Prof. Dr. med. Sevgi Tercanli, Basel

SGUM Präsidentin: Corina Canova

DEGUM Präsident: Kai-Sven Heling

ÖGUM Präsident: Hermann Kathrein

\section{S12 Wissenschaftliche Vortragssitzungen}

S12 Wi-Vo 01 MSK

Do. 15.11. 08:30 - 10:00 Shanghai 3

S16 Wi-Vo 02 Gynäkologie/Geburtshilfe I Do. 15.11. 08:30 - 10:00 Boston 3

S18 Wi-Vo 03 Gynäkologie/Geburtshilfe II Do. 15.11. 10:30 - 12:00 Shanghai 3

S20 Wi-Vo 04 Technik/Qualität/Sicherheit Do. 15.11. 10:30 - 12:00 Boston 3

S22 Wi-Vo 05 Kopf/Hals/Thorax Do. 15.11. 14:00 - 15:30 Shanghai 1

S25 Wi-Vo 06 Gynäkologie/Geburtshilfe III Do. 15.11. 14:00 - 15:30 Boston 3

S27 Wi-Vo 07 Kontrastmittel Fr. 16.11. 08:30 - 10:00 Shanghai 2

S30 Wi-Vo 08 Gynäkologie/Geburtshilfe IV Fr. 16.11. 08:30 - 10:00 Shanghai 3

S33 Wi-Vo 09 Pädiatrie/Urologie/Nephrologie Fr. 16.11. 08:30 - 10:00 Lima

S35 Wi-Vo 10 Abdomen Fr. 16.11. 10:30 - 12:00 Shanghai 2

S37 Wi-Vo 11 Gefäßdiagnostik Fr. 16.11. 10:20 - 12:00 Shanghai 3

S40 Wi-Vo 12 Gynäkologie/Geburtshilfe V Fr. 16.11. 13:00 - 14:30 Shanghai 2

S42 Wi-Vo 13 Mamma

Fr. 16.11. 13:00 - 14:30 Shanghai 3

\section{Posterpräsentationen}

\author{
P 01 Interdisziplinär \\ Do. 15.11. 12:00 - 13:30
}

\section{P 01.01 Generierung von Normwerten für die Acoustic Radiation Force Impulse (ARFI) Shear-Wave-Elastografie an der Achillessehne}

Autoren Wakker J ${ }^{1}$, Kratzer $W^{2}$, Gräter $T^{3}$, Schmidberger $]^{2}$

Institut 1 Klinik für Viszeral-, Thorax- und Gefäßchirurgie Stadtspital Triemli, Zürich; 2 Klinik für Innere Medizin I; 3 Klinik für diagnostische und interventionelle Radiologie/2 - 3 Universitätsklinikum Ulm DOI 10.1055/s-0038-1670364

Einführung Ziel der Studie war die Erhebung und Ermittlung von Normwerten für die Acoustic Radiation Force Impulse (ARFI) Shear-Wave-Elastografie für die Achillessehne.

Material und Methode Mit der VTIQ-Technik des Acuson S3000 TM (Siemens Healthcare, Erlangen, Germany) wurden an 182 Probanden die Scherwellengeschwindigkeit (SWV) in $\mathrm{m} / \mathrm{s}$ und der Durchmesser in $\mathrm{mm}$ bilateral an der Achillessehne erhoben. Die Darstellung der Sehne erfolgte in einem sagittalen Schallfenster, bei entspannter Sehne. Die erhobenen Parameter wurden hinsichtlich einer Korrelation zu den anthropometrischen Daten der Probanden, zwischen den Geschlechtern und verschiedenen Altersstufen, sowie auf anamnestisch erhobene Angaben wie Rauchen oder sportliche Aktivität untersucht. Resultate Wir ermittelten im sagittalen Schallfenster eine durchschnittliche SWV von $9,09 \pm 0,71 \mathrm{~m} / \mathrm{s}$ an der linken und $9,17 \pm 0,61 \mathrm{~m} / \mathrm{s}$ rechten Achillessehne $(P<0,05)$. Der Durchmesser lag links bei $4,7 \pm 0,9 \mathrm{~mm}$. Für die rechte Seite 4,8 $\pm 0,9 \mathrm{~mm}$. Im Gegensatz zu den gemessenen SWV wurden beim Durchmesser keine signifikanten Ergebnisse erhoben $(P>0,05)$. Es wurde für beide erhobenen Parameter keine Korrelation zwischen den Geschlechtern oder in Abhängigkeit des Alters festgestellt $(P>0,05)$.

Schlussfolgerungen Alter, Geschlecht und der BMI beeinflussen weder die Elastizität noch den Durchmesser der Achillessehne.

\section{P 01.02 Untersuchungen zur Ultraschalltransparenz von Schädeldachplastiken}

Autoren Mursch $\mathrm{K}^{1}$, Behnke-Mursch J ${ }^{1}$

Institut 1 Neurochirurgische Klinik, Zentralklinik Bad Berka DOI 10.1055/s-0038-1670365

Es wird im Allgemeinen angenommen, dass der menschliche Schädelknochen und auch Ersatzmaterialien nicht permeabel für diagnostischen Ultraschall sind.

Wir konnten an 2 Patienten beobachten, dass computerassistiert angefertigte frontoparietale PEEK (Polyetheretherketon) Schädeldachplastiken eine akzeptable Bildgebung intrakranieller parenchymatöser und vaskulärer Strukturen 
zuliessen. Es konnten sogar höhere Frequenzen als die für die transkranielle Sonografie gemeinhin verwendeten $2 \mathrm{MHZ}$ verwendet werden.

Weitere Untersuchungen am Phantom belegten die Ultraschalltransparenz von bis zu $4 \mathrm{~mm}$ starken PEEK-Plastiken, während beispielsweise Bioverit oder Palacos keine Bildgebung zuliessen.

Das Wissen um diese Möglichkeiten der Bildgebung kann in speziellen klinischen Situation sehr hilfreich sein. Sollte nach einer neurochirurgischen Operation eine computerassistiert angefertigte Plastik indiziert sein, könnte Entscheidung für das Material PEEK Vorteile haben.

\section{P 01.03 Rolle der Sonografie bei Darstellung von Menetekeln: Vermeidbarkeit klinischer Pyrrhussiege}

Autoren Seitz $\mathrm{KH}^{1}$

Institut 1 Klinik für Nephrologie, Pneumonologie, Rheumatologie, allgemeine Innere Medizin, I. Med. Klinik, Städtisches Klinikum Karlsruhe

DOI 10.1055/s-0038-1670366

Anhand dreier Patienten, bei denen nach komplexer Intervention (elektrophysiologische Ablation bei Vorhofflimmern, 2x TAVI-Anlage bei Aortenklappenstenose) sonografisch eine Lebermetastasierung aufgedeckt wurde, wird die Problematik organspezifischer Medizin ohne präinterventionelle Würdigung des Gesamtorganismus diskutiert, welche sich zwar im DRG-System rechnen mag, bzgl. der Gesamtprognose aber als nicht zielführend erweist (alle drei Patienten kamen in kurzem zeitlichen Verlauf ad exitum).

Schlussfolgerung Kompetente präinterventionelle sonografische Diagnostik wäre zur Vermeidung kostenintensiver, nicht prognoseverbessernder medizinischer Pyrrhussiege nahezulegen: Basale Bildgebung ist zu fordern und zu fördern.

\section{P 01.04 Schmerzhaftes Aneurysma der Arteria carotis externa}

Autoren Krishnabhakdi $S^{1}$

Institut 1 Gefäßchirurgie, Klinikum Herford

DOI 10.1055/s-0038-1670367

Patient und Methode Eine 74-jährige Patientin wurde notfallmäßig in unserer gefäßchirurgischen Klinik vorgestellt. Seit Monaten war der Patientin eine pulsierende Schwellung an der Halsseite aufgefallen, die an Größe zugenommen hatte. Seit wenigen Tagen verspürte sie Schmerzen und wurde vom Hausarzt noch am gleichen Tag zugewiesen. Klinisch fiel eine pulsierende, druckschmerzhafte Raumforderung auf. Triplex-Sonografisch konnte diese als teilthrombosiertes Aneurysma der Arteria carotis externa ohne Dissektion oder Zeichen einer Ruptur identifiziert werden. Das Aneurysma wurde operativ entfernt und die Strombahn durch Direktnaht rekonstruiert. Die Rekonstruktion war auch nach 6 Monaten offen, die Anastomose ohne Stenose oder Dilatation.

Schlussfolgerung Raumforderungen im Halsbereich sind häufig, pulsierende schmerzhafte dagegen sehr selten. Der seltene Fall eines schmerzhaften Aneurysma der Arteria carotis externa wird präsentiert. Die Sonografie als klinisch apparatives Verfahren der ersten Wahl bewies den klinischen Verdacht. Ohne weitere Diagnostik konnte festgelegt werden: 1. Die therapeutische Strategie (konservativ, konventionell operativ oder endovaskulär); 2. Der Zeitpunkt der Therapie. Informationen durch weitere Diagnostik und wichtige Differentialdiagnosen werden diskutiert.

\section{P 01.05 Multiple Komplikationen nach endovaskulärer Versorgung eines Poplitealaneurysmas}

Autoren Herzig $N^{1}$, Lorenz $\mathrm{P}^{2}$, Staub $\mathrm{D}^{2}$

Institut 1 Praxis für Gefäßkrankheiten, Rheinfelden; 2 Angiologische Klinik des Universitätsspital Basel

DOI 10.1055/s-0038-1670368

Einleitung Poplitealaneurysmen sind mit Abstand die häufigsten peripheren Aneurysmen. Endovaskuläre Therapieformen ersetzen auch bei den Poplitealaneurysmen zunehmend die offene Operation. Wir beschreiben einen Patienten mit ungünstigem Verlauf nach endovaskulärer Versorgung eines Poplitealaneurysmas.

Fallbeschreibung Bei dem 71-jährigen Patienten wurde 2013 ein femoro-popliteales Aneurysma links mittels Viabahnprothese versorgt. Einen Monat nach Anlage kam es zu einem akuten arteriellen Verschluss der Prothese, der distalen A. poplitea und Unterschenkelarterien. Mit einer lokalen Lysetherapie konnte der Verschluss rekanalisiert werden. Nach der Lysebehandlung kam es zu einer intrazerebralen Massenblutung, welche eine Kraniotomie mit Hämatomausräumung notwendig machte. Seither persistieren eine spastische Hemiparese, eine Hemianopsie sowie auch neuropsychologische Defizite. Zudem erlitt der Patient im Verlauf parazentrale Lungenembolien als Folge der Immobilisation, welche eine dauerhafte orale Antikoagulation notwendig machten. In den duplexsonografischen Verlaufskontrollen zeigt sich, trotz Endoprothese, eine kontinuierliche Größenprogredienz des Aneurysmasackes von initial 2,2 auf aktuell $4,2 \mathrm{~cm}$, ohne dass hierfür initial eine Ursache nachweisbar war. In der letzten Duplexsonografie konnte ein kleiner Seitenast als Endoleak dargestellt werden, der durch die Größenprogredienz zu einer Dislokation der distalen Endoprothese geführt hat. Geplant ist wegen der raschen Größenprogredienz eine operative Ausschaltung des Aneurysmas und Anlage eines Veneninterponates.

Kommentar Die endovaskuläre Behandlung von Poplitealaneurysmen wird als sichere und dauerhafte Therapieoption mit geringen Komplikationsraten und kürzerer Hospitalisationsdauer im Vergleich zur offenen Operation propagiert. Unser Fall zeigt, wie wichtig es ist, alle Vorteile und mögliche Komplikationen der einzelnen Therapieoptionen abzuwägen und mit den Patienten zu besprechen. Auch beim endovaskulär versorgten Poplitealaneurysma kann es zu einem Wachstum des Aneurysmasackes über Seitenäste (Analog Endoleak Typ 2 nach EVAR) kommen, sodass in diesem Fall gezielt danach gesucht werden sollte. Die Duplexsonografie, ggf. ergänzt durch eine KM-Gabe (CEUS) ist für die Diagnosestellung und postinterventionelle bzw. postoperative Verlaufskontrolle das Mittel der Wahl.

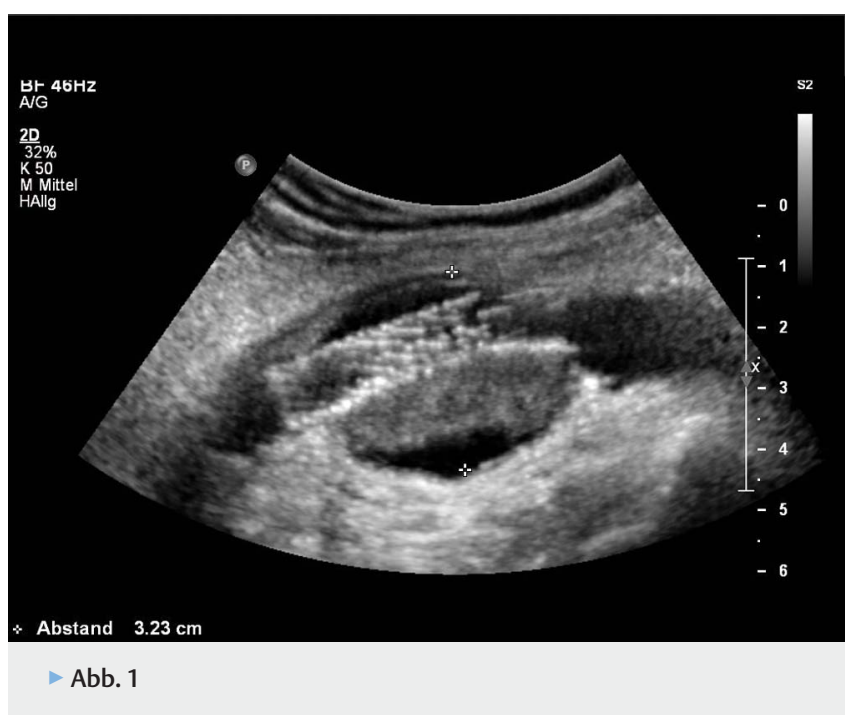




\section{P 01.06 Arrhythmie und akute Beinischämie - Diagnosefindung mittels FKDS}

Autoren Asbach $\mathrm{JC}^{1}$, Moussa $\mathrm{A}^{1}$, Bürger $\mathrm{T}^{2}$, Stegemann $\mathrm{E}^{1}$

Institut 1 Klinik für Allgemeine Innere Medizin und Angiologie; 2 Klinik für Gefäßchirurgie/ 1 - 2 Agaplesion Diakonie Kliniken Kassel

DOI 10.1055/s-0038-1670369

Einführung Bei Vorhofarrhythmien kann es als schwere Komplikation zum Auftreten einer akuten Extremitätenischämie kommen. Aufgrund der dringlichen Notwendigkeit einer raschen Reperfusion wird nicht immer eine Sonografie der Angiografie vorgeschaltet und so manchmal die „richtige“ Diagnose übersehen.

Fallbeschreibung Einweisung einer 66-jährigen Patientin mit akut aufgetretenem Schmerz des linken Beines sowie Arrhythmie. Motorik und Sensibilität sind noch erhalten, die Dopplersignale der Arteria (A.) poplitea nicht mehr, $\operatorname{der}$ A. tibialis anterior nur noch als kontinuierliches Signal ableitbar.

Diagnostik/Therapie Aufgrund der Akuität der Situation und der sofortigen Verfügbarkeit der Angiografieanlage erfolgt unmittelbar eine intraarterielle Angiografie. Dort „spontan rekanalisierte Embolie“ der A. poplitea mit Restthromben, Planung einer Intervention am Folgetag, da kein Schmerz mehr besteht und nicht über Nacht lysiert werden soll. Am Folgetag dann Angioplastie des linken Beines mit ausgezeichnetem Resultat, während der Intervention plötzlich vagale Reaktion und Schmerz der Leiste. In der Computertomografie Nachweis eines retroperitonealen Hämatoms, kein Transfusionsbedarf. Eine Woche nach Entlassung dann Wiedervorstellung wegen erneuter progredienter Schmerzen des linken Beines, Gehstrecke ca $50 \mathrm{~m}$. Sonografisch Nachweis einer Riesenzellarteriitis der AFS bds, der Aorta, der Beckenarterien sowie der A. axillaris rechts.

Diskussion Die sofortige Reperfusion des Beines stand dermaßen im Vordergrund, dass sämtliche Hinweise auf das Vorliegen einer anderen Erkrankung als der eines embolischen Verschlusses übersehen wurden. Retrospektiv haben mehrere Kontrollmechanismen versagt und die Befundung des vorliegenden Bildmaterials war nur auf eine Fragestellung fokussiert erfolgt. Wegweisend für die tatsächliche Diagnose war letztendlich die Sonografie, welche auch zur Verlaufskontrolle der Erkrankung herangezogen wird.

\section{P 01.07 Der rettende Würgegriff}

\section{Autoren Pitarch D}

Institut 1 Cabinet de médecine générale Demetrio Pitarch, Boncourt DOI 10.1055/s-0038-1670370

Ein 22-jähriger junger Mann stellt sich 3 Wochen nachdem er beim Raitiaitiai (Karneval der Jugend im Kanton Jura) von einem angetrunkenen Kumpel gewürgt wurde wegen peristierenden Schluckbeschwerden vor. Klinisch findet sich kein Hämatom und kein palpabler Befund im gewürgten Bereich. Das Röntgen zeigt keine ossäre Läsion im Bereich des Zungenbeins. Im Hals-US normale Verhältnisse bis auf eine $1,55 \mathrm{~cm} \times 0,83 \mathrm{~cm} \times 1,95 \mathrm{~cm}$ große, von einem Halo umgebene Läsion in der rechten ansonst normalen Schilddrüse. Der Patient wird informiert, dass es ich um ein residuelles Hämatom handeln könnte und wird ein Monat später zur Kontrolle einbestellt. Der bei Folgeuntersuchung asymptomatische Patient präsentiert einen sonografisch unveränderten Befund. Die bei der beigezogenen Endokrinologin erfolgte Feinnadelpunktion ergibt die histologische Verdachts-Diagnose eiunes pilären Karzinoms, weswegen nun für den 24.5.2018 eine totale Thyroidektomie geplant ist.

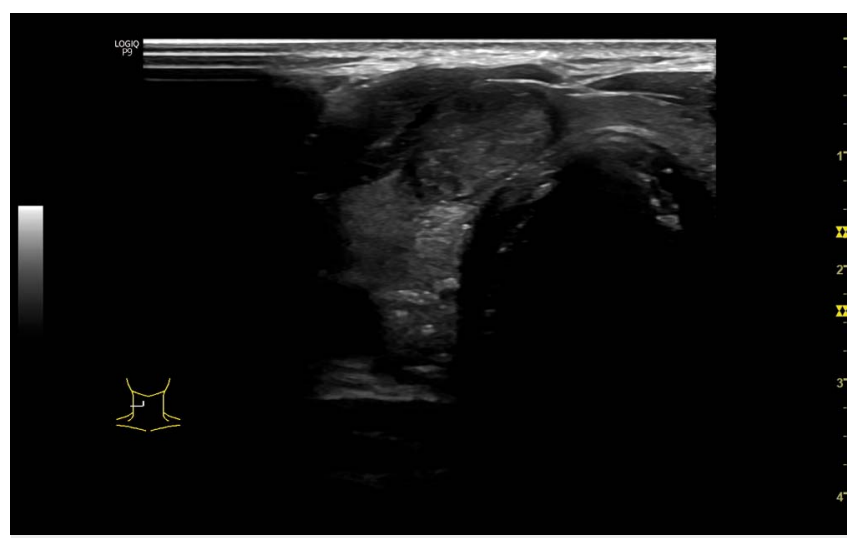

Abb. 1

\section{P 01.08 Sonografie einer einseitigen Recurrensparese bei papillärem Schilddrüsenkarzinom}

Autoren Wowra $\mathrm{T}^{1}$, Debatin $\mathrm{KM}^{1}$

Institut 1 Klinik für Kinder- und Jugendmedizin, Universitätsklinikum Ulm DOI 10.1055/s-0038-1670371

Patient und Methode Ein sieben Jahre altes Mädchen mit gesicherter Diagnose eines papillären Schilddrüsenkarzinoms und bekannten Lymphknoten- und Lungenmetastasen wird in unserer Klinik zur weiteren Therapie vorgestellt. Neben einer Dyspnoe mit Sauerstoffbedarf bis $8 \mathrm{l} / \mathrm{min}$ über eine Maske bei ausgeprägter Struma, fällt vor allem eine starke Kachexie auf. Es liegen zum Zeitpunkt der Aufnahme keine sonograpischen Vorbefunde vor. Im Ultraschall der Halspartie stellt sich die Schilddrüse vergrößert mit stellenweise unscharfer Randbegrenzung dar. Ubiquitär findet sich beidseits eine gesprenkelte sehr echoreiche Grundtextur, normales Schilddrüsengewebe ist kaum noch abgrenzbar. Parathyroidal fallen ebenfalls beidseitig Lymphknoten von ähnlicher echoreicher Sonomorphe, Metastasen entsprechend, auf. Das Tracheallumen imponiert eingeengt (Bild 1). Die rechte Vena jugularis interna ist komprimiert, der Blutfluss ensprechend einer beginnenden Thrombosierung (Bild 2). Eine Therapie mit Lenvatinib (Multibler Kinase Inhibitor) reduziert den Sauerstoffbedarf innerhalb weniger Tage drastisch und sorgt für eine deutliche klinische Verbesserung. In einer Kontrollcomputertomografie etwa einen Monat nach Therapiebeginn sind die Metastasen rückläufig. Sonografisch zeigt sich der oben beschriebene Schilddrüsenlokalbefund bis zu diesem Zeitpunkt unverändert. Da das Mädchen jedoch über Heiserkeit klagt wird der Larynx in die folgende Sonografie eine Woche nach Aufnahme miteinbezogen und zeigt nur linksseitig eine Bewegung des Stimmlippenapparates. Damit konnte die Diagnose einer rechtsseitigen Recurrensparaese gestellt werden.

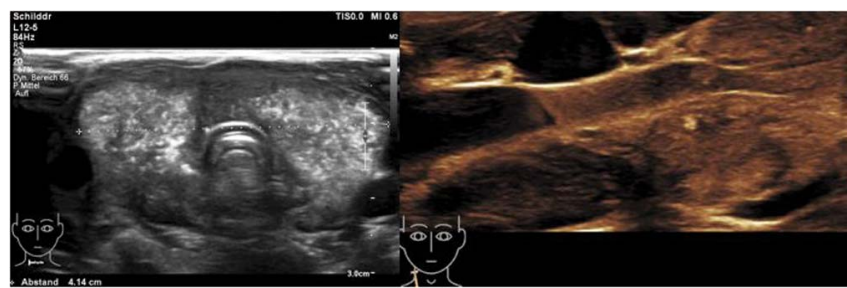

Abb. 1

Schlussfolgerung Die nicht seltene Beteiligung des Nervus vagus je nach Stadium eines papillären Schilddrüsenkarzinoms ist bekannt. Die direkte und indirekte Laryngoskopie und videostroboskopische Untersuchungen des Kehlkopfes entsprechen dabei dem Goldstandard. Insbesondere bei agitierten Kindern ist die Untersuchung oft nicht ohne Sedierung durchführbar. Dieser Fall zeigt die sonografische Diagnose einer rechtseitigen Recurrensparese. 


\section{P 01.09 Primäre Frakturdiagnostik mittels Ultraschall in der Notaufnahme}

Autoren Schöll $\mathrm{E}^{1}$, Bollinger $\mathrm{N}^{1}$

Institut 1 ORTHO-NOTFALL, Merian Iselin Klinik für Orthopädie und Chirurgie DOI 10.1055/s-0038-1670372

Einführung Ultraschalluntersuchung zur primären Frakturdiagnostik wird seit 2002 in der Handchirurgie verwendet und seit 2005 auch in anderen traumatologischen Disziplinen praktiziert. Während die Methode für Läsionen der Weichteile zum Goldstandard geworden ist, spielt sie in der Frakturdiagnostik eher noch eine untergeordnete Rolle, obwohl neuere Publikationen eine vergleichbare Sensitivität mit konventioneller Röntgendiagnostik sogar für tiefer gelegene Knochen zeigen. Der Point-of-care-Ultraschall entwickelt sich in jüngerer Vergangenheit zu einem universellen Hilfsmittel, welches bereits beim Transport von Patienten oder in der Notaufnahme eine akkurate Diagnostik ermöglicht.

Patienten und Methode Anhand von drei Fallbeispielen wird die Erstdiagnostik von Frakturen in unserer Notaufnahme mittels einer Linearsonde (14 MHz, MINDRAY TE7) im Vergleich zum später durchgeführten konventionellen Röntgen als primäre Frakturdiagnostik beschrieben.
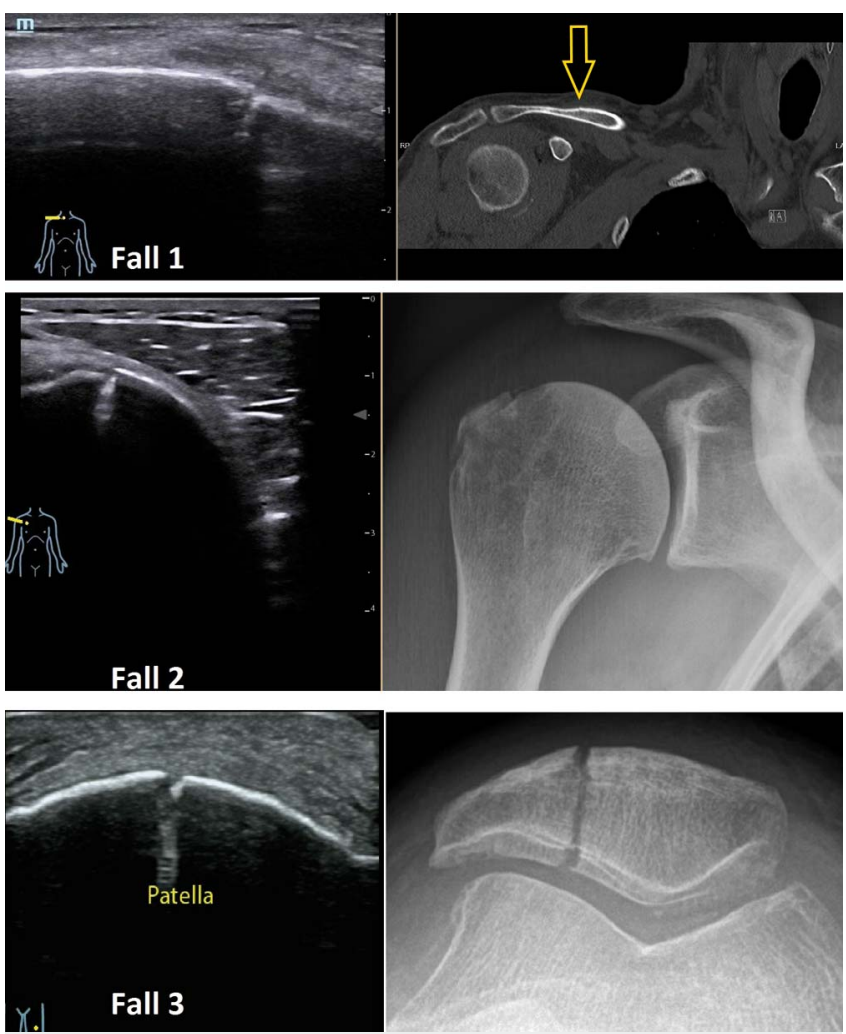

Abb. 1

Fall 1 50-jähriger Patient nach Sturz vor einer Woche auf die rechte Seite. Das initiale Thorax-Röntgen hatte keine Frakturen und keinen Pneumothorax gezeigt. Wegen unveränderter Schmerzen im Bereich der Clavicula sowie im rechten Thorax wurde er uns zugewiesen. Die Ultraschalldiagnostik zeigte eine undislozierte Claviculafraktur (Abb. 1) sowie Rippenfrakturen 5 und 6 rechts in der Medioclavicularlinie, welche später erst in der CT verifiziert werden konnten.

Fall 2 48-jähriger Patient, welcher 9 Tage zuvor ausgerutscht war und sich mit dem rechten Arm abgefangen hatte, seither Schmerzen im Bereich der langen Bizepssehne und bei Aussenrotation. Die Ultraschalldiagnostik konnte die gering dislozierte Fraktur des Tuberculum majus klar zeigen (Abb. 2), im Röntgenbild wurde die Diagnose einer Tendinosis calcarea ohne Frakturverdacht gestellt.
Fall 3 75-jährige Patientin, welche gestolpert und direkt auf das rechte Knie gefallen war. Die primäre Sonografie und Röntgendiagnostik kamen zum gleichen Ergebnis der Patellalängsfraktur (Abb. 3).

Schlussfolgerung Muskuloskelettaler Ultraschall als primäre Bildgebung in der Frakturdiagnostik ist ein suffizientes Werkzeug zur korrekten Diagnosestellung in der Notfallaufnahme. Jegliche oberflächennahe Knochen, wie die in dieser Publikation dargestellten Clavicula- und Patellafrakturen, sind mit hochauflösenden Schallköpfen wesentlich einfacher darstellbar als tiefere. Unabdingbar für die Interpretation des Bildes ist die Kenntnis der Knochenanatomie sowie des umgebenden Bindegewebes.

\section{P 01.10 Intraoperative Sonografische Lavagekontrolle - ein Fallbericht bei schwerer therapierefraktärer Alveolarproteinose}

Autoren Rüdiger $]^{1}$, Schmid $\mathrm{P}^{2}$, Tamm $\mathrm{M}^{1}$

Institut 1 Pneumologie; 2 Anästhesie/1-2 Universitätsspital Basel

DOI 10.1055/s-0038-1670373

Die Alveolarproteinose ist eine schwere Krankheit durch einen Defekt in der GM-CSF Wirkung. Es kommt zum Auskleiden der Alveolen und Atemwege mit Protein und in der Folge zu einer Gasaustauschstörung der Lungen. Ultima Ratio ist die zweitzeitige Ein-Lungenlavage über Stunden in Vollnarkose mit 30 - 40 Liter $\mathrm{NaCl}$ 0,9\%. Hier berichten wir über die Anwendung der transthorakalen Sonografie zur Überprüfung der Lavageausbreitung.

Methoden Es erfolgte eine Intubationsanästhesie mit totaler intravenöser Anästhesie und Relaxation. Die Intubation erfolgte mit einem Doppellumentubus links (DLT Viva Sight 39), wodurch sowohl eine videoassistierte DLT-Einlage als auch eine kontinuierliche Überwachung der Tubuslage ermöglicht wurde. Die Lungenlavage wurde in einer Einlungenventilation durchgefürt, wobei die nicht belüftete Lunge mit 30-40 Liter $\mathrm{NaCl} 09 \%$ (gewärmt) schrittweise gefüllt und entleert wurde.

Ergebnisse Im Fall einer schlechten Sättigung unter Einlungenventilation wurde intra-operativ sonografisch ein ventraler Pneumothorax beidseits ausgeschlossen. Im Verlauf zeigte sich eine persistierende Hypoxämie bei im seitenvergleich abgeschwächtem Atemgeräusch links, so dass die Sonografie wiederholt wurde. Es zeigte sich links weiterhin ein normales Pleuragleiten und als Ursache für das verstärkte Atemgeräusch rechts einen wassergefüllten Oberlappen. Die Sonografie wurde auf Grund der Lungendarstellung zur Verlaufsbeurteilung der Lavage herangezogen. Bei der ersten Lavage zeigte die Sonografie eine fasst vollständig gewässerte Lunge rechts. In der zweiten Lavage (7,5 Monate später) mit deutlicher Progredienz konnte der Unterlappen sonografisch nur teilweise und der Mittellappen fast nicht mit Wasser gefüllt werden. Es wurde zwischenzeitlich eine Seitenlagerung durchgeführt, wodurch der Unterlappen der Lavage besser zugänglich wurde.

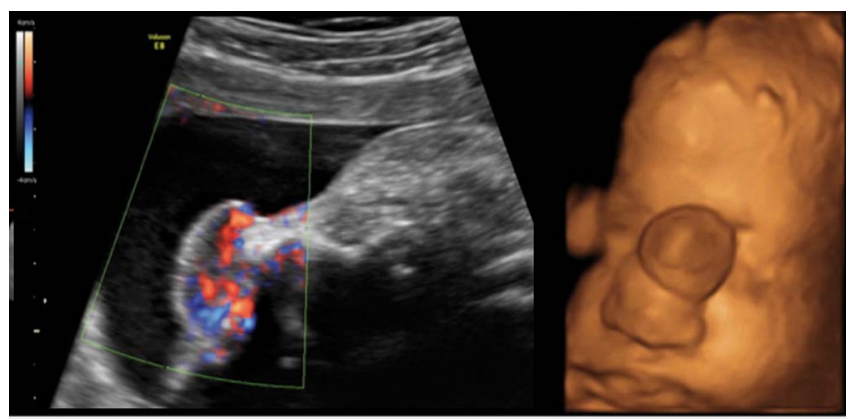

Abb. 1

Schlussfolgerung Die Sonografie ist eine einfache und sichere Beurteilungsmöglichkeit, um bei intubierten Patienten therapeutische pulmonale Flüssigkeitsveränderungen darzustellen. Wir schlagen vor, die Sonografie zur Verlaufsbeurteilung bei Totaler Lungenlavage und anderen Eingriffen mit Spülung der Lunge heranzuziehen. 


\section{P 01.11 Point-of-care-Ultrasound (POCUS) - Möglichkeiten des mobilen Ultraschalls bei Hausbesuchen in ländlichen Gebieten - Eine Machbarkeitsstudie}

Autoren Frauendorf $\mathrm{V}^{1}$, Thierock $\mathrm{M}^{2}$, Parthe $\mathrm{H}^{3}$, Schmailzl $\mathrm{KJ}{ }^{1}$, Nuernberg D Institut 1 Ruppiger Kliniken, Hochschulklinikum der Medizinischen Hochschule Brandenburg; 2 Medizinisches Versorgungszentrum Außenstelle Wildberg, OGD Neuruppin; 3 Gemeinschafstpraxis H. Parthe \& E. Jahnke Groß Pankow

DOI 10.1055/s-0038-1670374

Einleitung Aufgrund des demografischen Wandels, der Urbanisierung und des Ärztemangels droht insbesondere in ländlichen Gebieten Deutschlands eine Unterversorgung der alternden Bevölkerung. Durch die Weiterentwicklung portabler Ultraschallgeräte können diagnostische und therapeutische Möglichkeiten bei Hausbesuchen besser ausgenutzt werden. Schon heute kann der Einsatz von POCUS in der außerklinischen Ersteinschätzung von Traumata wie auch in Bereichen der Palliativmedizin durch Therapiemaßnahmen wie z.B. Ergusspunktionen zu einer verbesserten Patientenversorgung beitragen (Nuernberg D. et al 2015 Z Gastroenterol; Nuernberg D. et al 2017 Z Gastroenterol).

Materialen und Methoden Im Vordergrund stand die Testung eines Schulungsprogrammes auch für ultraschallunerfahrene Ärzte. Nach einem 3 stündigen Workshop, welcher eine Studien- und Gerätevorstellung, das Erkennen einfacher sonografischer Befunde und Punktionsübungen am Phantom beinhaltete, folgte eine zweiwöchige Lernphase mit individuell angeleiteten Patientenuntersuchungen mit einem portablen Ultraschallgerät der Firma GE (Vscan Extend). Danach nutzten die geschulten Ärzte POCUS in ihrer prästationären allgemein- wie palliativmedizinischen Versorgung über 6 Wochen. Leitsymptome, Untersuchungsergebnisse und resultierende Behandlungsänderungen (z. B. Akutinterventionen oder Medikamentenanpassungen) wurden in Form eines einheitlichen Erhebungsbogens dokumentiert.

Resultate Von 96 in Nordwestbrandenburg angeschriebenen Haus- und/oder Palliativärzten beteiligten sich 13 an dem zuvor beschriebenen Schulungsprogramm. Für eine Studienteilnahme mit Anwendung in der ärztlichen Praxis konnten insgesamt 10 Ärzte gewonnen werden. Hiervon wiesen 7 Teilnehmer ausreichende Ultraschallkenntnisse auf. Für 3 ultraschallunerfahrene Ärzte wurde ein intensiverer Support durch Supervision ambulanter Untersuchungen angeboten. Während einer durchschnittlichen Nutzungsdauer von 6 Wochen wurden bisher 35 Patienten bei Hausbesuchen mittels Ultraschall untersucht.

Schlussfolgerungen Es konnte gezeigt werden, dass ein individuell angepasstes Schulungsprogramm geeignet ist auch ultraschallunerfahrenen Ärzten eine sichere Handhabung in POCUS zur Beantwortung einfacher klinischer Fragegestellungen zu ermöglichen. Durch die hohen Nutzungsbereitschaft der teilnehmenden Ärzte erhoffen wir uns durch Auswertung der Erhebungsbögen auch einen messbaren Einfluss auf die Patientenversorgung in ländlichen Gebieten zeigen zu können.

\section{P 01.12 Lebervenenvarixknoten bei Rechtsherzbelastung}

Autoren Seitz $\mathrm{KH}^{1}$

Institut 1 Abteilung für Nephrologie, Pneumonologie,Rheumatologie, allgemeine Innere Medizin, I. Medizinische Klinik, Städtisches Klinikum Karlsruhe

DOI 10.1055/s-0038-1670375

Eine 67-jährige Patientin mit ausgeprägter Rechtsherzbelastung nach Mitralund Aortenklappenersatz wird zur operativen Sanierung einer $5 \mathrm{~cm}$ messenden eingebluteten Lebercyste/DD vaskulären Malformation zufolge externer Voruntersuchung zugewiesen.

Farbduplexsonografisch zeigt sich eine nicht parenchymgedeckte cystoide Formation mit „Waschmaschinen-Peristaltik“ wie bei Aneurysma spurium bei
Pendelfluss einer bis in die Peripherie erweiterten kommunizierenden Lebervene. In externer und interner CT wurde die Läsion als KM-aufnehmende Raumforderung, DD eingebluteter Tumor, gedeutet. Kontrastmittelsonografisch, im CT und angiografisch kein Nachweis eines arteriellen zuführenden Gefäßes (MRT aufgrund PM nicht durchführbar). Bei im Operationssitus wie eingeblutete Cyste wirkendem Befund weist die Histologie nach Zystenwandresektion und Unterbindung eines kräftigen Gefäßes eine Phlebektasie nach.

Schlussfolgerung FKDS und CEUS legten präoperativ den Verdacht auf einen Lebervenen- varixknoten bei nicht konklusiver KM-CT-Studie nahe. Ein ähnlich gelagerter Präzedenzfall ist der Literatur nicht zu entnehmen.

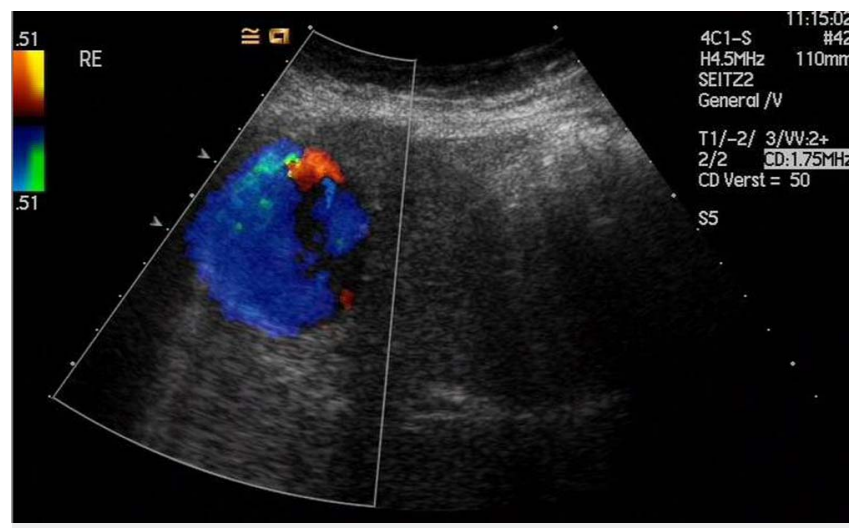

Abb. 1

\section{P 01.13 Angiosarkom des Fußrückens}

Autoren Mertens J $]^{1}$, Krishnabhakdi S

Institut 1 Gefäßchirurgie, Klinikum Herford

DOI 10.1055/s-0038-1670376

Patient und Methode Ein 57-jähriger männlicher Patient stellt sich wegen einer Raumforderung im Bereich des Fußrückens vor. Wiederholte Sklerosierungen wegen symptomatischer AV-Fisteln waren auswärts ohne Erfolg durchgeführt worden. Die etwa $10 \mathrm{~cm}$ große, derbe, nicht druckschmerzhafte Schwellung auf dem Fußrücken war sonografisch inhomogen mit echofreien Inseln und soliden Gewebsanteilen in Bezug auf die Dignität suspekt. In der farbkodierten Duplex- und pw-Doppler-Sonografie wurde ein Konglomerat aus AV-Fisteln identifiziert und der dringende Verdacht auf ein Angiosarkom gestellt. Weitere Schnittbildverfahren erhärteten den Verdacht, der bioptisch gesichert wurde.

Schlussfolgerung Tumorbefunde des Fußrückens sind selten und fallen klinisch auf. Ein ungewöhnlicher Tumor am Fußrücken wurde sonografisch als dringend malignitätsverdächtig identifiziert. Die Sonografie als leicht verfügbares klinisch-apparatives Instrument ermöglichte eine zeitnahe Diagnosestellung und Einleitung der stadiengerechten Therapie. Die Literatur wird diskutiert.

\section{P 01.14 Case report - detection of simultaneous liver lesions: multiples hemangiomata and amoebiasis}

Authors Todorov $\mathrm{S}^{1}$, Firmann $\mathrm{M}^{1}$, Burnand $\mathrm{J}^{1}$, Erard $\mathrm{V}^{2}$, Schiemann $\mathrm{U}^{1}$ Institute 1 Department of Internal Medicine, HFR-Riaz; 2 Department of infectious diseases HFR-Fribourg DOI 10.1055/s-0038-1670377

Amoebiasis remains a significant cause of morbidity and mortality (up to 100,000 deaths/y worldwide). Amoebic liver abscess is the most common extra-intestinal manifestation.

We report a case of a 51-year-old male patient with a history of abdominal discomfort and abdominal bloating, weight lost and fever up to $39^{\circ} \mathrm{C}$. He has travelled in India for one week 4 months ago. Laboratory tests show increased 
CRP (221 mg/l) and leukocytosis (15.0 G/l) without eosinophilia. Liver and pancreatic enzymes were normal. Urinary, stool and blood cultures were negative for bacterial origines. Abdominal ultrasound showed several hyperechogenic hemangiomata (liver segments V, VI and VII) and a hypoechogenic lesion (liver segment 6), compatible with neoplasia, atypique hemangioma or abscess (Fig. 1). An abdominal MRI confirms the diagnosis of hemangiomata and suggested an liver abscess of $4,5 \mathrm{~cm}$. Two serology tests (ELISA, IFAT) confirmed the diagnosis of amoebiasis. Treatment of metronidazole and paromomycine was initiated and the patient clinically recovered. Radiological control six months later showed a complete regression of the liver abscess.

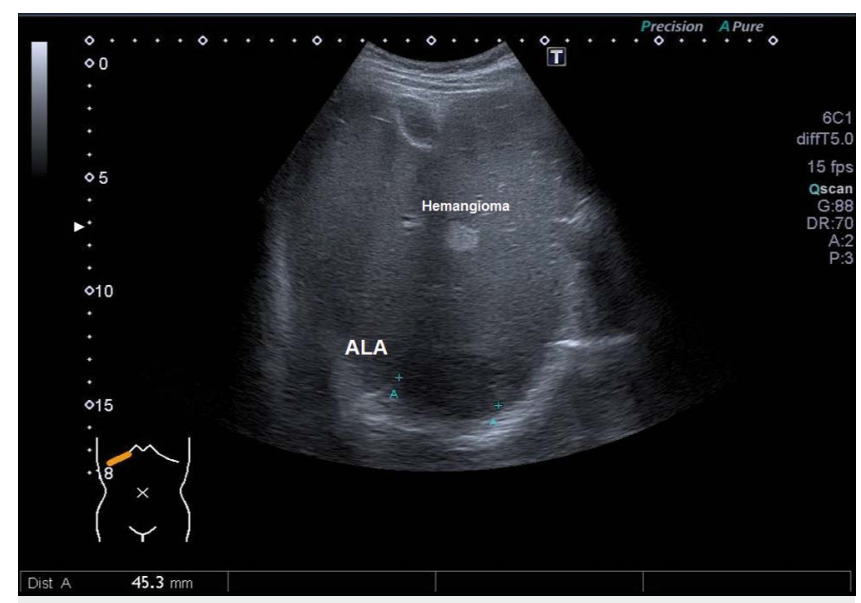

Pig. 1

Conclusion Amoebiatic liver abscess is a potential complication of infection by entamoeba histolytica. The presence of simultaneous liver hemangiomata in our case was tricky, potentially leading to misunderstand the life-threatening diagnosis. Abdominal ultrasound and the travel history represent a key moment of the diagnostic process.

\section{P 01.15 Der transrektale Ultaschall (TRUS) in der urologischen Praxis}

Autoren Enzmann $T^{1}$

Institut 1 Klinik für Urologie, Medizinische Hochschule Brandenburg -

Campus Städtisches Klinikum Brandenburg

DOI 10.1055/s-0038-1670378

Der transrektale Ultraschall bleibt trotz moderner Bildgebungsverfahren wie z.B. die multiparametrische MRT oder die PSMA-PET-CT ein fester Bestandteil in der urologischen Versorgungspraxis. Dies wird illustriert an Hand der Indikationen des TRUS:

- Blasenwanddickenmessung bei der Obstruktion,

- Bewertung des prostataspezifischen Antigen,

- Staging beim Prostatakarzinom,

- entzündlichen Prozesse der Prostata,

- Anastomosenkontrolle nach radikaler Prostatektomie und damit Ersatz des Cystogramms.
P 02 Gynäkologie/Geburtshilfe

Fr. 16.11. 11:00 - 12:30

P 02.01 Einführung des Ultraschalls in die Schwangerschaftsvorsorge in der Kilimanjaro Region, Tansania

Autoren Neuenschwander D ${ }^{1}$, Gysel $W^{1}$

Institut $1 \mathrm{SmW}$ Stiftung für medizinischen Wissenstransfer

DOI 10.1055/s-0038-1670379

Die Stiftung für medizinischen Wissenstransfer SmW bietet seit 2010 regelmässig Ultraschallkurse in Geburtshilfe, Abdomen- und Notfallsonografie in Ostafrika (Kenia und Tansania) an. Falls notwendig werden den Spitälern unserer Kursteilnehmenden unter geringer Kostenbeteiligung und gemäss MoU gute gebrauchte Ultraschall-Geräte vermittelt.

Seit 2014 wurden in der Region Kilimanjaro, Tansania, spezielle geburtshilfliche Kurse in Zusammenarbeit mit den lokalen Instanzen durchgeführt: Einführungskurs über vier Tage, nach einigen Monaten viertägiger Abschlusskurs mit Prüfung zum Erlangen des „Certificate of Completion in Pregnancy Screening Scan Week 20-24“, dazwischen supervidierte Untersuchungen in den lokalen Institutionen und Gruppentrainings. Das Screening ist fokussiert auf die Festlegung von Geburtstermin, Mehrlingsschwangerschaften, Plazentalokalisation und Fruchtwasserbeurteilung. Durchgeführt wird das Screening im Rahmen der in Tansania konstenfreien Schwangerschafsvorsorge. möglichst im 2. Trimenon vor der 24. Woche. Auf weitergehende morphologische Beurteilung wird gezielt verzichtet.

Zentral war die Gewinnung möglichst gut ausgebildeter lokaler Health Professionals zur Mitarbeit, die Unterstützung durch lokale Gesundheitsinstitutionen und durch die Behörden. Aktuell sind alle 7 Distrikte der Region Kilimanjaro mit 25 Screening Centers am Programm beteiligt, 19 davon mit einem durch SmW vermittelten Ultraschall-Gerät.

Monatlich wird SmW durch den regionalen Radiographer über die durchgeführten Untersuchungen in den 25 Zentren informiert.

\section{P 02.02 Prädiktive Wertigkeit fetaler Überwachungsparameter bei früher Intrauteriner Wachstumsrestriktion (fIUGR) - retrospektive Kohortenanalyse}

Autoren Schneider $U^{1}$, Trinks $L^{1}$, Dawczynski $K^{2}$, Proquittè $H^{2}$, Schleußner $E^{1}$ Institut 1 Klinik für Geburtsmedizin, Universitätsklinikum Jena (UKJ); 2 Klinik für Kinder- und Jugendmedizin, Sektion Neonatologie und Pädiatrische Intensivtherapie, UK]

DOI 10.1055/s-0038-1670380

Fragestellung fIUGR < 34 SSW definiert ein spezielles Risikokollektiv in dem die Entscheidung hinsichtlich Prolongation vs. Entbindung das Risiko der Frühgeburt gegen das der intrauterinen Schädigung abwägt. Im eigenen Kollektiv sollen retrospektiv Parameter der fetalen Überwachung hinsichtlich ihrer Prädiktivität für perinatale Morbidität und Mortalität bewertet werden.

Methode Aus der Geburtskohorte der Jahre 2006-2014 der Universitätsfrauenklinik Jena (gesamt 12496 Geb.) wurden anhand der Perinatalstatistik alle Fälle mit einem Geburtsgewicht < 10. Gewichtsperzentile (1145 Lebendgeburten oder Totgeburten $>500 \mathrm{~g}$ ) ermittelt. 84 Kinder wurden vor $34+0$ SSW geboren (0,67\%). Es erfolgte die Erhebung von Basisdaten aus der Perinatalerhebung, des letzten Ultraschallbefundes vor Geburt und eine retrospektive CTG-Auswertung des letzten präpartalen CTGs. Perinatales und neonatales Outcome wurden anhand kombinierter Endpunkte (Mortalität: IUFT und neonatales Versterben $<8$ Lebenswochen; ,Azidose': APGAR5<7/ pHNA<7,1/BE-10, neonatale Reanimationsmaßnahmen, Verlegung NICU; neonatale Komplikationen: IVH/NEC/BPD/PTX/PVL/PDA) zusammengefasst. Resultate 80 Kinder wurden lebend geboren, von diesen verstarben im weiteren 18 , die Gesamtmortalität betrug $27,5 \%$, die neonatale Mortalität 22,5\%. 
Die Sectiorate betrug 95\%. 36,9\% zeigten Auffälligkeiten im Parameter ,Azidose‘, Reanimationsmaßnahmen erfolgten bei 66,7\%, alle Lebendgeborenen Kinder wurden auf NICU verlegt. Die Prävalenz auffälliger sonografischer Überwachungsparameter (Biometrie und Doppler) lag bis auf wenige Ausnahmen bei $>75 \%$, dementsprechend hohe Sensitivitäten negativen Outcomes bei geringer Spezifität waren zu erfassen. Die beste Vorhersagekraft besaß ein PVIV DV> 95Perc. (20/59 Fälle; Sens./Spez./ppV/npV/OR[CI], Angaben gerundet \%): ,Azidose‘: 47/73/45/74/2,4 [0,8-7,4]; neonatale Komplikationen 54/72/20/85/3 [0,8-10,5]; Mortalität 75/77/45/92/9,8 [2,3-42,7]. Vergleichbar hohe Spezifität und npV zeigte nur der Parameter ,suspektes CTG، bei geringerem ppV. Ein fetales Schätzgewicht <2,5Perc. hatte mit $26 \%$ den höchsten ppV neonataler Komplikationen.

Diskussion Wie aus den Ergebnissen der TRUFFLE Studie zu erwarten war, lassen auch in unserem retrospektiven Kollektiv biometrische und arterielle Dopplerparameter keine Prädikation negativen Outcomes bei früher IUGR zu. Es findet sich zudem die erwartete Gestationsalterabhängigkeit fetaler Morbidität und Mortalität sowie eine Assoziation zu besonders niedrigem Geburtsgewicht.

\section{P 02.03 Intrauteriner Fruchttod - retrospektive 7-Jahre Analyse mit dem Fokus auf maternal anamnestische Risikofaktoren}

\section{Autoren Vogeler $\mathrm{F}^{1}$, Guttmann $\mathrm{M}^{2}$, Dombrowski $\mathrm{F}^{3}$, Ruhland $\mathrm{F}^{1}$ Institut 1 Gsund Kompetenzzentrum Stralsund; 2 Geburtshilfe Hanseklinikum Stralsund; 3 Institut für Pathologie Universität Greifswald DOI 10.1055/s-0038-1670381}

Einführung Definition der Totgeburt n. Bundesdeutschen Recht: Geburtsgewicht von mindestens $500 \mathrm{~g}$ ohne Lebenszeichen/Pulsieren der Nabelschnur. Derzeit liegen noch nicht genügend Daten zur Ursachenanalyse vor. Auf der Basis dieses Kollektivs sollen weiterführende Erkenntnisse hinsichtlich prädiktiver Faktoren mit dem Fokus auf maternal anamnestische Risikofaktoren ermittelt werden.

Material und Methoden In einem 7-jährigen Zeitraum von 01.01.201031.12.2017 wurden bei 6912 Entbindungen im Hanseklinikum Stralsund (perinataler Schwerpunkt Level III) per Geburtendokumentationssystem sowie Geburtenbücher alle IUFT eruiert. Fälle nach medizinisch indiziertem Schwangerschaftsabbruch wurden nicht einbegzogen.

Resultate 26 IUFT (Einlingsschwangerschaft: 25/Geminigravidität:1) bei 6912 Entbindungen; korrigierte Totgeburtenrate 3,7/1000 Geburten Schwangerschaftswoche:

$65 \%(n=17)>30$. SSW - hiervon $47 \%(n=8)$ reife Totgeburten

Terminüberschreitungen $12 \%(n=3)$ der Fälle

$8 \%(n=2)<25$. SSW

Geburtsgewicht: Median 1863,3 g; Spannweite $510 \mathrm{~g}$ bis $5140 \mathrm{~g}$, SGA-Feten >32. SSW in $39 \%(n=10)$

Geschlechtverhältnis: 51\% männlich zu 49\% weiblichen Feten. Keine Neigung zur Totgeburt nach Geschlecht

Todesursache: Festlegung der Haupttodesursache nach Obduktions-Plazentabefunde/klinischen Befunden war in 50\% $(n=13)$ der Fälle möglich.

Plazentainsuffizienz 38\% ( $n=5)$, vorzeitige Plazentalösung 23\% ( $n=3)$, Nabelschnurkomplikationen 23\% ( $n=3)$, fetale Fehlbildung 15\% ( $n=2)$ Hypothetisch: nicht diagnostizierte late IUGR bei den SGA-Feten nach der 32. SSW $(n=10)$

II. analytische Statistik -maternale anamnestische Risikofaktoren

Alter: Median: 28,5 Jahre; Spannweite 19 bis 44 Jahre; $12 \%(n=3) \geq 35$ Jahre Parität: $58 \%(n=15)$ Nullipara, Parität $>4$ in $8 \%(n=2)$

BMI vor der Schwangerschaft: Median 27,9 Jahren; Spannweite 20,1 bis 41,5; $65 \%(n=17)$ der Fälle BMI > 25; $59 \%(n=10)$ der adipösen Schwangeren waren Nullipara.

Schlussfolgerung Die Assoziation maternales Alter/IUFD ist etabliert. Andersen et al. beschreiben diese als J-förmigen Kurvenverlauf; erhöht bei Teenager- schwangerschaften sowie ab dem 35 Lebensalter. In dieser Analyse jedoch traten $88 \%$ Fälle in der "low-risk“ Gruppe des maternalen Alters auf. Wie von Eschler beschrieben sprechen auch diese Daten für ein erhöhtes Risiko bei Nullipara. Analog zur „matrnal and fetal risk fators for stillbirth:population based study" stellt Adipositas eineiner der maternalen Hauptrisikofaktoren dar.

\section{P 02.04 Polyhydramnion mit unauffälligen Fruchtwasserelektrolyten mi postnataler Polyurie und Elektrolytstörung}

Autoren Weichert $A^{1}$, Henrich $W^{1}$, Bührer $C^{2}$, Garten $L^{2}$, Mühlbacher $T^{2}$

Institut 1 Klinik für Geburtsmedizin; 2 Klinik für Neonatologie/ 1 - 2 Charité Universitätsmedizin Berlin

DOI 10.1055/s-0038-1670382

Hintergrund Das Bartter-Syndrom umfasst eine Gruppe hereditärer Tubulopathien mit Chloridverlust, Hypokaliämie, Hyponatriämie und Polyurie. Gemeinsame pränatale Präsentation ist ein u. U. massives Polyhydramnion, das häufig zur Frühgeburtlichkeit führt. Besonders ausgeprägt tritt dieses bei dem X-chromosomal rezessivem Bartter Syndrom Typ V (MAGED 2 Genmuatationen) auf.

Fallbericht Wir berichten von einer 29-jährigen I-Gravida, 0-Para, die sich in 26 SSW mit einem massiven Polyhyhdramnion vorstellte. Bei deutlichen materialen Beschwerden erfolgte eine Amniondrainage, wobei die Elektrolyte des Fruchtwassers analysiert und eine molekulargenetische Analyse veranlasst wurden. Die Elektrolyte ergaben einen unauffälligen Befund. Bei therapieresistenten vorzeitigen Wehen wurde ein männlicher Neonat in 27 SSW. entbunden. Postnatal zeigte sich mit transientem Verlauf eine Polyurie und mit schwerem Salzverlustsyndrom. Die Behandlung mit Indomethacin resultierte in einer Normalisierung der Diurese und des Volumenbedarfs bei langfristiger Elektrolyt-Substitution. Die Indomethacin-Therapie wurde 3 Wochen vor dem errechneten Termin beendet, ohne dass es zu einem Rezidiv kam. Die molekulargenetische Untersuchung der klassischen Bartter-Gene inklusive des Typ $\checkmark$ verursachenden MAGED2 (melanoma-associated antigen D2) war unauffällig, jedoch konnte eine hemizygote Mutation der Splice-Donor-Site des Introns 10 im MAGED 2 Gen nachgewiesen werden, die zu einer reduzierten MAGED 2-Expression führt.

Diskussion Das Bartter-Syndrom gehört zu den Differentialdiagnosen des Polyhydramnions. Während bei den meisten Formen (Typ I-IV) eine lebenslange Indomethacingabe erforderlich ist, kommt es beim MAGED 2-assoziierten Typ $\mathrm{V}$ (mit besonders ausgeprägtem Polyhydramnion) nach 1-2 Monaten zu einer Spontanheilung.

Schlussfolgerung Die molekulargenetische Diagnostik sollte nicht nur auf die reine Exon-Analyse der Bartter-Gene abzielen, sondern auch die Betrachtung der relevanten Spleiß-Regionen einschließen.

\section{P 02.05 Fetus in Fetu: Sacrococcygealer Fetus in Fetu mit Steißbeinteratom}

Autoren Froehlich $\mathrm{C}^{1}$, Woeckel $\mathrm{A}^{1}$, Rehn $\mathrm{M}^{1}$, Meyer $\mathrm{T}^{1}$

Institut 1 Geburtshilfe, Universitätsklinik Würzburg

DOI 10.1055/s-0038-1670383

Einleitung Fetus in Fetu ist ein sehr seltenes Bild eines embryonalen Tumors. Die Inzidenz beträgt 1/500.000 Lebendgeburten. Definitionsgemäß ist ein Fetus in Fetu von einem Teratom abzugrenzen durch das Vorhandensein eines Achsenskeletts und zeichnet sich durch eine hohen Grad an Differenzierung/ Organentwicklung aus.

Methodik Die Erstvorstellung der Patientin erfolgte in der 24. SSW. Sonomorphologisch zeigte sich ein rießiger sacrococcygealer Tumor $12 \times 10 \times 9 \mathrm{~cm}$ Größe mit erkennbaren knöchernen Strukturen, Schädelkalotte und Achsenskelett ohne Herzaktion, entsprechend einem Fetus in Fetu. Es erfolgten regelmäßige sonografische Verlaufskontrollen. In der $34+6$ SSW kam es bei vorzeitigem Blasensprung zur Entbindung per $\|^{\circ}$ Sectio. 
Resultate Das postnatale MRT zeigte deutliche Anlagen von Wirbelsäule, Schädel, Thoraxhöhle und Extremitäten mit Muskulatur sowie weit verzweigte intratumorale Gefäße mit direktem Anschluss an die iliaca Strombahn rechts. Am 7. Lebenstag erfolgte die Resektion des Tumors mit Sphinkter- und Beckenbodenrekonstruktion. Der postoperative Verlauf zeigte sich komplikationslos. Histologisch aufgearbeitet wurde ein $1078 \mathrm{~g}$ schweres Präparat. Dabei konnten mehrere Extremitäten mit Fingern, ein Achsenskelett, Rippen sowie eine Schädelkalotte mit einem differenzierten Gehirn dargestellt werden. Als Besonderheit neben der Fetus in Fetu Morphologie zeigten sich auch Teile eine kleinzystischen Teratoms. Der Tumor wurde R0 reseziert und zeigte keinen Anhalt für Malignität.

Schlussfolgerung Bei einem Fetus in Fetu handelt es sich um eine äußerst seltene Entität eines embryonalen Tumors. Hauptprädilektionsstelle ist das Abdomen, aber auch intrakranielle, intrapulmonale oder Tumoren im Steißbeinbereich sind in der Literatur beschrieben. Die Pathogenese ist unklar. Als mögliche Pathophysiologie wird eine abnormale Embryogenese mit einem monochorialen parasitären Zwilling diskutiert. Eine weitere Theorie beschreibt den Fetus in Fetu als "fetiformes" hochorganisiertes, differenziertes, reifes Teratom. Beide Theorien werden in der Literatur diskutiert, die Theorie einer Zwillingsanlage gilt als wahrscheinlicher. In diesem Fall konnten neben dem Fetus in Fetu auch Teile eines kleinzystischen reifen Teratoms nachgewiesen werden.

\section{P 02.06 Nichtimmunologischer Hydrops fetalis bei EPHB4-Mutation}

Autoren Becker $\mathrm{WH}^{1}$, Hempel $\mathrm{M}^{2}$, vd Wense $\mathrm{A}^{3}$

Institut 1 Zentrum für Pränatalmedizin an der Elbe, Hamburg; 2 Institut für Humangenetik, Universitätsklinikum Hamburg Eppendorf; 3 Neonatologie und Pädiatrische Intensivmedizin, Altonaer Kinderkrankenhaus Hamburg DOI 10.1055/s-0038-1670384

Patienten und Methode Wir berichten über eine 37-jährige II. gravida I. para mit unauffälliger Eigen- und Familienanamnese. Die erste Schwangerschaft aus einer anderen Partnerschaft verlief unauffällig. Die Blutgruppe ist $A B$ rh neg. Bei SSW $13+0$ erfolgte die Erstvorstellung mit unauffälliger Ersttrimesteruntersuchung: NT 2,00 mm, adjustiertes Risiko für Trisomie 21 1: 2862, Trisomie 18 1: 7306, Trisomie 13 1: 22827 (Ultraschall mit Zusatzmarkern und maternaler Serumbiochemie (beta hCG, PAPP-A, PIGF), FMF UK Algorithmus 2012). Risiko für Präeklampsie < SSW 34+0 1: 5731. Bei SSW 19+0 erfolgte die Zweittrimesteruntersuchung. Hier zeigte sich sonografisch ein milder Pleuraerguß re., ansonsten unauffälliger Scan. Eine Woche später war dieser rückläufig. TORCH-Serologie und Antikörper-Suchtest waren unauffällig. Eine Amniocentese ergab folgende Ergebnisse: 46,XX. Mutationsanalyse Noonan negativ (PTPN11, BRAF, CBL, KRAS, NRAS, SHOC2, SOS1, RAAF1, RIT1, RRAS). SMN1 neg. Mutationsanalyse Thalassämie neg. (HBA1, HBA2, HBB). RHD neg. Array-CGH unauffällig arr $(1-22, X) \times 2$. Bei SSW $28+6$ zeigten sich ein moderater Pleuraerguß re., Polyhydramnion und ein fetales Schätzgewicht auf der 95er Perzentile. Ein oGTT war unauffällig.

Bei SSW 30+4 sahen wir einen ausgeprägten generalisierten Hydrops fetalis mit Zwerchfellinversion beidseits und stark alterierten Dopplerindizes. Wir führten eine prompte Fetalpunktion mit Pleurashunting re. durch. Postinterventionell kam es zu einer anhaltenden Bradycardie. Bei V. a. Dekompensation sofortige Klinikeinweisung zur Notsectio. Die Kindsdaten: Gewicht $3000 \mathrm{~g}$, APGAR 4/7/7, pH 7,27, base excess $-4,1 \mathrm{mmol} / \mathrm{l}$. Nach 60 Tagen neonatologischer Intensivtherapie verstarb das Kind. Das Whole Exom Sequencing zeigte eine EPHB4-Thyrosinkinase-Inaktivator-Mutation (HiSeq2500 system, Illumina, San Diego, CA, USA).

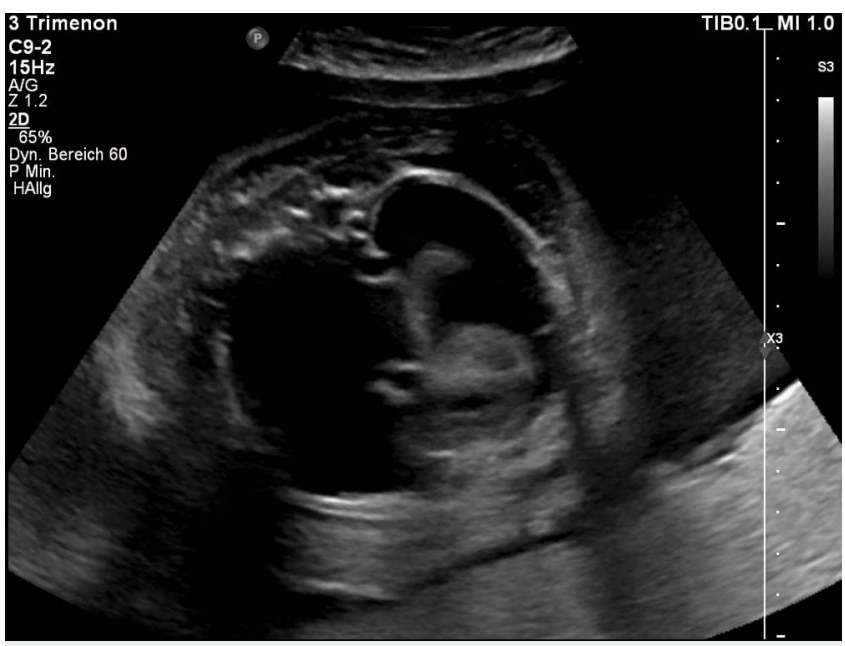

Abb. 1

Schlussfolgerung Der nichtimmunologische Hydrops fetalis ist eine schwerwiegende Erkrankung unterschiedlicher Genese. Eine Klärung der Ursache ist oft aufwendig und gelingt nicht immer. Sie sollte aber immer angestrebt werden, da sie sich gegebenenfalls positiv auf einen Trauerprozess auswirkt und bei der Planung einer Folgeschwangerschaft helfen kann.

Die hier gefundene EPHB4-Mutation stellt eine seltene Primär Lymphatische Dysplasie mit autosomal dominantem Erbgang dar. Die Ursache des Hydrops fetalis kann somit als geklärt gelten. In interdisziplinärer Zusammenarbeit konnten wir einen Indexpatienten identifizieren.

\section{P 02.07 Veränderung im ANXA5 Promotor-Gen mit rezidivierenden massiven Retardierungen in Schwangerschaften und mögliche Therapieoptionen}

Autoren Delius $\mathrm{M}^{1}$, Rogenhofer $\mathrm{N}^{1}$, Kleinhempel $\mathrm{A}^{2}$, Hasbargen $\mathrm{U}^{1}$, Mahner $S^{1}$, Hübener $C^{1}$

Institut 1 Klinik und Poliklinik für Frauenheilkunde und Geburtshilfe;

2 Institut für Laboratoriumsmedizin/ 1 - 2 Klinikum der Universität München, LMU München

DOI 10.1055/s-0038-1670385

Einführung Ein erhöhtes Risiko für rezidivierende Spontanaborte ist bei maternaler oder paternaler Trägerschaft des M2/ANXA Haplotyps beschrieben. Studienergebnisse weisen darauf hin, dass in diesen Fällen eine Therapie mit niedermolakularen Heparinen vorteilhaft ist (Rogenhofer et al. 2016).

Patienten und Methode Vorgestellt wird eine 32-j. afghanische, nicht konsanguine Patientin nach 3 Schwangerschaften. Die erste Schwangerschaft hatte die Patientin 2014 mit dem IUFT eines retardierten Feten in der 21. SSW verloren. Die zweite Schwangerschaft endete 2016 mit einem IUFT bei 27+1 SSW bei einem ebenfalls schwer retardiertem Feten ( $480 \mathrm{~g})$. Die Patientin stellte sich erstmalig in der 23. SSW mit bereits deutlicher Retardierung und pathologischem Doppler A. umbilicalis vor. Nach ausführlichen interdisziplinären Gesprächen mit dem Ehepaar und den Kollegen der Neonatologie wurde ein exspektatives Vorgehen mit ambulanten Kontrollen vereinbart; bei 26+6 SSW zeigte sich eine negative A-Welle im Ductus venosus, bei geschätzten $470 \mathrm{~g}$ des Kindes. Eine RDS-Prophylaxe wurde begonnen, die Entbindung für den nächsten Tag geplant. Am folgenden Tag wurde der IUFT des Feten festgestellt. In keiner der Schwangerschaften hatte die Patientin frühzeitig ASS erhalten.

In und nach der 2. Schwangerschaft erfolgte eine erweiterte Abklärung: TORCH neg., Pathologie: hypotropher Fet, keine Fehlbildungen, ausgedehnte Plazentainfarkte (>70\%); Abklärung RSA Labor: Gerinnung unauffällig, kein Lupus, Schilddrüse unauffällig, MTHFR und Prothrombin: keine Mutation, ANXA5-Promotor Haplotyp: heterozygote Trägerin des M1-Haplotyps im Promotorbereich des ANXA5-Gens sowie heterozygoter Trägerin des 19G>A- 
Austauschs; Bei dem Ehemann wurde exakt die gleiche Konstellation des ANXA5-Promotor Haplotyps gefunden. In der 3. komplikationslosen Schwangerschaft wurde die Patientin 2017 per pimärer Sectio 39+0 SSW bei BEL von einem männlichen Feten mit $2840 \mathrm{~g}$ entbunden. Die Pat. hatte ASS $100 \mathrm{mg}$ und Clexane 40 ab positiver Herzaktion sowie Pentaeritrithyltetranitrat (PETN) als individuellen Heilversuch ab 22+2 SSW $50 \mathrm{mg}$ tgl. bis 36+6 SSW erhalten. Schlussfolgerung Die hier bei beiden Eltern vorliegende Konstellation des ANXA5-Promotor Haplotyps ist so bisher nicht in der Literatur beschrieben worden. Die vorgestellte Kasuistik lässt jedoch vermuten, dass es sich hier um eine besondere Mutation mit pathologischer Bedeutung und therapeutischer Konsequenz handelt.

\section{P 02.08 Abdominelle Fibromatose vom Desmoid-Typ in graviditate - watch and wait}

Autoren Hübener $C^{1}$, Lindner $L^{2}$, Rjosk-Dendorfer $D^{3}$, Gießelmann $B^{4}$, Mahner $\mathrm{S}^{1}$, Hasbargen $\mathrm{U}^{1}$, Delius $\mathrm{M}^{1}$

Institut 1 Klinik und Poliklinik für Frauenheilkunde und Geburtshilfe;

2 Medizinische Klinik und Poliklinik III; 3 Klinik und Poliklinik für Radiologie;

4 Gynäkologie am Stachus, München/1 - 3 Klinikum der Universität München,

Ludwig-Maximilians-Universität München

DOI 10.1055/s-0038-1670386

Patienten und Methode Eine 36-jährige G3 P1 mit Diabetes mellitus Typ I bemerkte im Verlauf der Schwangerschaft eine rundliche Raumforderung im Bereich der Bauchdecke mit deutlichem Größenwachstum und wurde mit der Verdachtsdiagnose Hämatom DD Myom zur weiteren Diagnostik und Therapie überwiesen. In der Sonografie zeigte sich eine solide echoarme, inhomogene, glatt begrenzte Raumforderung der Bauchdecke von $10 \times 6 \times 8 \mathrm{~cm}$ mit zentral echoreichem Septum mit zuführendem Gefäß. Der Tumor war vom Uterus abzugrenzen und extraperitoneal gelegen. Eine MRT-Untersuchung bestätigte den sonografischen Befund. Bei V.a. Desmoidtumor wurde zunächst im interdisziplinären Konsens unter engmaschiger Kontrolle zugewartet. In der 26. Schwangerschaftswoche (SSW) betrug das Volumen $270 \mathrm{ml}$, in der 36. SSW $390 \mathrm{ml}$. Nach einer unauffälligen Spontangeburt erfolgte eine Histologiesicherung per Stanzbiospie, welche eine abdominelle Fibromatose vom Desmoid-Typ ergab. Man entschied sich für die Fortsetzung des exspektativen Vorgehens, das Tumorvolumen nahm im Verlauf sukzessive ab (nach sechs Monaten $250 \mathrm{ml}$, nach 12 Monaten $145 \mathrm{ml}$, nach 18 Monaten $70 \mathrm{ml}$, nach 24 Monaten $20 \mathrm{ml}$ und nach 4 Jahren $15 \mathrm{ml}$ ). Eine Resektion wurde bei Beschwerdefreiheit von der Patientin nicht gewünscht.

Schlussfolgerung Desmoidtumore oder desmoide Fibromatosen sind seltene monoklonale Weichteiltumoren fibroblastären Ursprungs mit großem lokalinfiltrativen Wachstumspotential ohne Metastasierungrisiko. Sie treten entweder sporadisch mit einer Frequenz von 1-5 auf eine Million pro Jahr oder assoziiert mit der FAP (Familiäre adenomatöse Polyposis) auf. Insbesondere in der Schwangerschaft ist ein rasches Wachstum beschrieben, Trauma und Hyperöstrogenämie scheinen eine Rolle in der Pathogenese zu spielen. Nach der Schwangerschaft tritt in einem relevanten Anteil der Fälle eine Spontanregression auf. Insbesondere bei einer neu auftretenden Raumforderung der Abdominalwand während einer Schwangerschaft mit gut abgrenzbaren Tumorgrenzen muss an die seltene Diagnose eines Desmoidtumors gedacht und vor chirurgischer Intervention bei Beschwerdefreiheit die mögliche Spontanregression nach Schwangerschaft und Wochenbett abgewartet werden. Während früher die chirurgische Sanierung mit ausgedehnter Resektion als Primärtherapie gesehen wurde, ist nun nach europäischem Consensus ein exspektatives Vorgehen nach stanzbioptischer Histologiegewinnung der primäre Ansatz (watchful waiting über initial 1 - 2 Jahre).

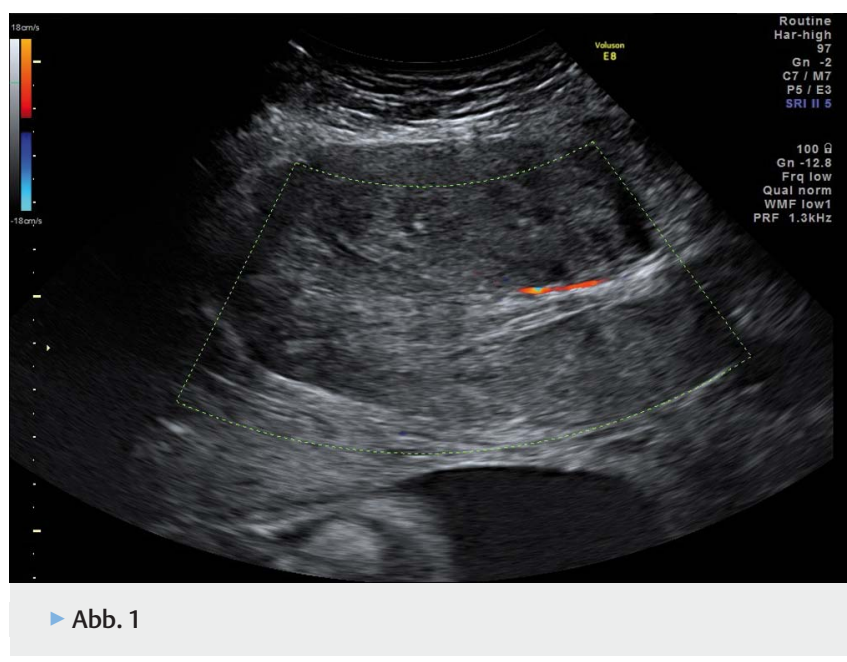

\section{P 02.09 Cell free fetal DNA fraction in the first trimester: a sign for abnormally invasive placenta?}

Authors Wertaschnigg $\mathrm{D}^{1}$, Lucovnik $\mathrm{M}^{2}$, Klieser $\mathrm{E}^{3}$, Huber-Katamay J', Moertl $\mathrm{MG}^{4}$

Institute 1 Department of Obstetrics and Gynecology; 2 Department of Perinatology, University Medical Centre Ljubljana; 3 Department of Pathology; 4 Department of Obstetrics and Gynecology, Terry care center for

Perinatology, Medical Center, Klagenfurt/1,3 Salzburger Landeskrankenhaus, Paracelsus Medical University

DOI 10.1055/s-0038-1670387

We report about a pregnancy complicated by an abnormally invasive placenta (AIP) treated with a planned caesarean-hysterectomy at 34 weeks GA (Gestational Age). At 12+5 weeks GA noninvasive prenatal testing (NIPT) showed a low risk for all trisomies, but cell free-fetal (cff) DNA fraction was highly elevated with $35.3 \%$. We repeated NIPT at $26+6$ weeks GA. At that time the cff DNA fraction was still very increased with $26.1 \%$.

Median cff DNA fraction at 10+6 GA is reported with $10.2 \%$, between 10 and 21 weeks gestation, fetal fraction (ff) increases at $0.10 \%$ per week. After the first half of pregnancy the ff increases at a rate of $1 \%$ per week. So far there have been published inconsistent data about increased level of cff DNA as a biomarker for AIP during the third trimester. No data exist about the first trimester. Our case supports the theory that cff DNA fraction as biomarker for AIP maybe better in the first trimester than later in pregnancy. Our patient with AIP had a very elevated level of cff DNA fraction of $35.3 \%$ at the end of the first trimester. According to the published data by Wang E. et al. the median level of cff DNA fraction in normal pregnancies at this GA is only around $10.4 \%$. Probably due to increased apoptosis of placental cells in the second half of pregnancy, median cff DNA-fraction increases about $1 \%$ per week and is around $25 \%$ at 34 wks GA. These results of our case support and Samuel's theory that cff DNA fraction as biomarker for AIP maybe better in the first trimester than later in pregnancy. Our case report and previous data from Sekizawa et al. should, therefore, prompt further studies on diagnostic value of cff DNA fraction in early pregnancy. Abnormally invasive Placenta (AIP) is one of the most dangerous conditions associated with pregnancy, because hemorrhage may result in severe acute maternal morbidity. Cff DNA as a possible marker of subsequent AIP could help to improve accuracy of prenatal diagnosis of morbidly invasive placenta. 


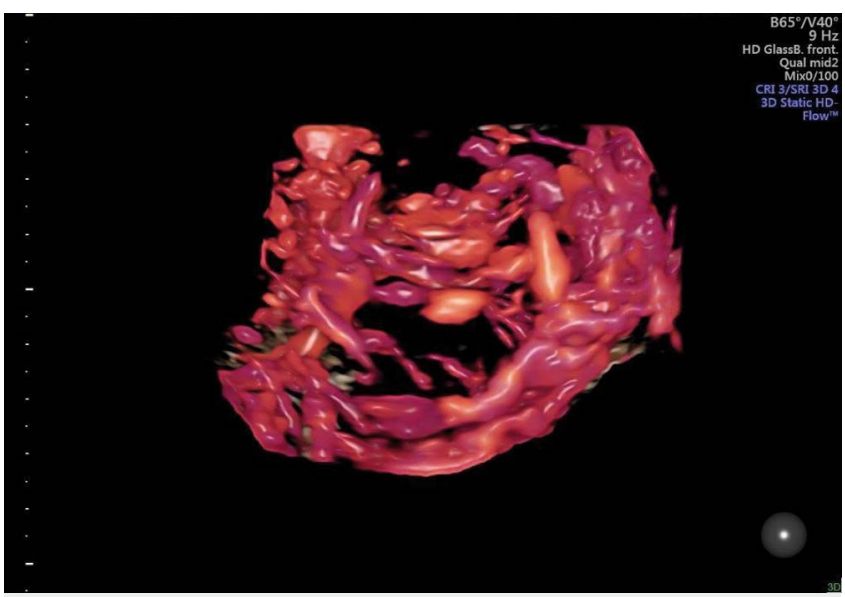

Fig. 1 3D-Ultrasound-Color-Doppler (HDlive technology) and Glass Body rendering shows intraplacental hypervascularity: complex, irregular arrangement of numerous placental vessels, exhibiting tortuous courses and varying calibers 11.

\section{P 02.10 Die Differentialdiagnose eines Gesichtstumors und die Diagnostik mittels Ultraschall und MRT}

Autoren Al Naimi $\mathrm{A}^{1}$, Spahn $\mathrm{S}^{1}$, Bahlmann $\mathrm{F}^{1}$

Institut 1 Frauenklinik, Bürgerhospital Frankfurt

DOI 10.1055/s-0038-1670388

Patienten und Methode 30-jährige GII P0 Patientin wurde uns in der 33+5 SSW beim Verdacht auf Gesichtstumor eingewiesen. Sowohl NIPD als auch Organscreening ergaben in dieser Schwangerschaft unauffällige Befunde. Die orientierte Ultraschalluntersuchung bestätigte einen $1,6 \mathrm{~cm}$ großen rundlichen Befund zwischen Nasenwurzel und innerer Augenwinkel links, welcher durchblutet war. Die Differentialdiagnose an Hand des Ultraschallbefundes beinhaltete: frontale Enzephalocele, Hämangiom (wahrscheinlich), Proboscis lateralis, Hautanhängsel.

MRT Unauffällige Hirnstruktur kann eine Enzephalocele 100\% ausschließen. Linksseitg paramedian an der Nase befindet sich eine Struktur ohne Kontakt zum Neurocranum im Bereich des Gesichtsschädels, die mit einer Proboscis lateralis vereinbar ist. Fruchtwasser ist darstellbar nur im rechten Nasengang was eventuell für Fehlbildung der linken Nasengang hindeutet.

Ergebnis Aufgrund der Uneinstimmung zwischen den sonografischen und tomografischen Diagnosen (Hämangiom VS Proboscis lateralis) wurde zur Optimierung der peripartalen Versorgung eine primäre Sectio geplant. Das geborene Mädchen hat einen $2 \mathrm{~cm}$ großen rundlicher Tumor der Nasenwurzel (weich, blass-livide, himbeerartige Oberfläche) mit freien Nasengängen. Die postpartale Diagnose eines Hämangioms wurde sowohl klinisch als auch mit MRT bestätigt, und die weitere konservative Behandlung mit Propranolol wurde angeleitet.

Schlussfolgerung Die pränatale Darstellung eines Tumors am fetalen Gesicht mittels Ultraschall ist leicht. Eine genaue Bestimmung der Diagnose pränatal ist trotzdem kompliziert, da die sonografischen und tomografischen Befunde unterschiedliche Aussagen bezüglich der Differentialdiagnose ergeben können. Die Abgrenzung der möglichen Diagnosen ist notwendig für die Optimierung des perinatalen Managements.

\section{P 02.11 Angebornenes progeroides Syndrom aka Wiedemann-Rautenstrauch-Syndrom (WRS) - Eine seltene Ursache einer fetalen Wachstumsrestriktion}

Autoren Hartge $D^{1}$, Gembicki $M^{1}$, Weichert J

Institut 1 Abteilung für Pränatalmedizin, Klinik für Frauenheilkunde und Geburtshilfe, Universitätsklinikum Schleswig-Holstein, Campus Lübeck DOI 10.1055/s-0038-1670389

Wir beschreiben die pränatale Diagnostik eines Falles mit Wiedemann-Rautenstrauch-Syndrom (WRS): Eine 34 IIGOP wurde uns bei fetaler Wachstumsrestriktion in der $32+1$ SSW ins Zentrum zugewiesen. Es zeigte sich ein dystropher männlicher Fet mit einem SG von $1043 \mathrm{~g}$ (<3. Perz.) bei unauffälligen fetalen und maternalen Dopplerparametern. Die Schädelsuturen und Fontanellen waren deutlich erweitert, eine okzipitale Enzephalozele konnte nicht ausgeschlossen werden. Es zeigten sich eine Mikrognathie verbunden mit einer Mittelgesichtshypoplasie, eine prominente Nase, prominente Unterlider, sowie ein tiefer Ohransatz bds. Das Unterhautfettgewebe erschien wenig ausgebildet. Es erfolgte die invasive Diagnostik, die eine Kombination der bereits von den (gesunden) Eltern bekannten Veränderung zeigte (Dupl15q3.3 („maternal“) Del22q11.22q11.23 („paternal“)).

Das Paar entschied sich die Schwangerschaft nicht fortzuführen und es wurde nach entsprechenden Maßnahmen der Fetozid durchgeführt und das Kind in der 33. SSW geboren. Postparatal wurden die erhobenen Befunde und die Diagnose eines WRS bestätigt.

Das hier berichtete WRS ist ein seit 1977 bekanntes seltenes Syndrom mit bislang 18 bestätigten Fällen, die zumeist neonatal beschrieben wurden. Feten mit diesem angeborenen autosomal-rezessiv vererbten progeroiden Syndrom können sonografisch typischerweise mit einer fetalen Wachstumsrestriktion, einer generalisierten Lipodystrophie, tiefliegenden Augäpfeln bei fehlendem Orbitalfettkissen mit konsekutiv prominenten Unterlidern, tiefem Ohransatz, einer Mikrogathie, einer hakenförmige Nase und erweiterten Schädelnähten auffallen. Betroffene Kinder zeigen neben neurologischen und anderen Beeinträchtigungen eine deutliche Wachstumseinschränkung und eine verkürzte Lebenserwartung mit einem hohen Risiko des Versterbens innerhalb der ersten Lebensjahre.

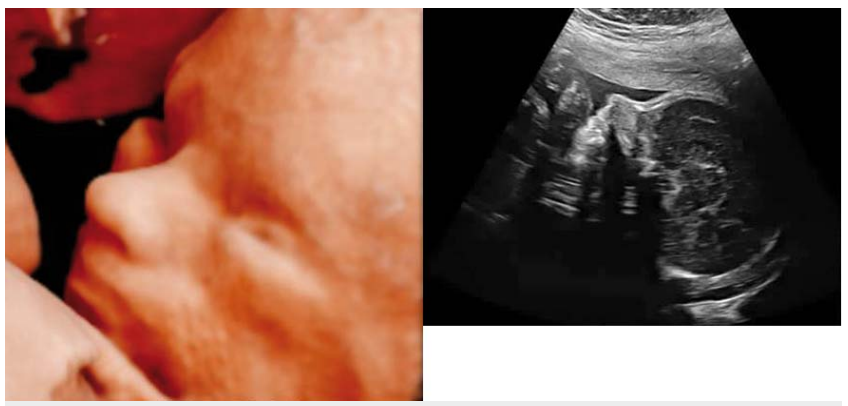

Abb. 1

\section{P 02.12 Interdisziplinäres prä- und postnatales Management bei komplexer urogenitaler Malformation}

Autoren Voigt $C^{1}$, Schneider $U^{1}$, Dawczynski $K^{2}$, Proquitte $H^{2}$, Eckoldt $F^{3}$, Mentzel $\mathrm{HJ}^{4}$, Schleußner $\mathrm{E}^{1}$

Institut 1 Klinik für Geburtsmedizin; 2 Klinik für Kinder- und Jugendmedizin, Sektion Neonatologie; 3 Klinik für Kinderchirurgie; 4 Institut für

Diagnostische und Interventionelle Radiologie, Sektion Kinderradiologie/ 1 - 4 Universitätsklinikum Jena

DOI 10.1055/s-0038-1670390

Kasuistik 29-jährige Patientin, G I P 0, stellte sich in der $29+0$. SSW erstmals mit neu aufgetretenem Hydrops fetalis mit ausgeprägtem Aszites, Hydrotho- 
rax und generalisiertem Hautödem vor. Sonografisch zeigte sich eine zystische Struktur im kleinen Becken, die am ehesten dem dilatiertem fetalem Enddarm zuzuordnen war. Die fetale Echokardiografie war unauffällig, das Kind bot keine Herzrhythmusstörungen. Ein immunologisches, infektiologisches oder anderweitig fetal-anämisches Korrelat wurden ausgeschlossen. Die Zytogenetik war unauffällig. Zur Diagnostik und symptomatischen Besserung der oberen Einflussstauung erfolgte eine Entlastungspunktion des fetalen Aszites $(320 \mathrm{ml})$. Eine ergänzende MRT-Untersuchung bestätigte den V.a. einen Hydrops fetalis auf der Basis einer Kloakenfehlbildung mit Sinus urogenitalis und retrograd austretendem Uro-Aszites über die Tubae uterinae. Während einer Kontrolluntersuchung kam es zur akuten fetalen Dekompensation. Das kleine Mädchen wurde in der $31+6$. SSW per Sectio geboren. Bei initial schwierigen Belüftungsverhältnissen erfolgte die Punktion des „septierten Aszites“ $(180 \mathrm{ml})$, nachfolgend gelang die Stabilisation des Neugeborenen. In der Bildgebung stellte sich der Situs als Sinus urogenitalis mit sekundärer Hydrokolpos - durch einen sehr weit distal gelegenen Fistelgang zwischen Urethra und Vagina und einer Analfistel - dar. Es folgten die Anlage eines Zystostomas und die Entlastung der Hydrokolpos durch eine Pigtail-Drainage. Der postnatale Verlauf wurde durch rezidivierende Pyelonephritiden erschwert. Im weiteren Verlauf zeigte das Neugeborene eine nicht altersgerechte motorische Grundaktivität mit insgesamt wenig Spontanmotorik. Die Entwicklung einer hypertonen zentralen Koordinationsstörung ist wahrscheinlich. Es besteht der Verdacht auf Seh- und Hörstörungen. Im Alter von 8 Wochen konnte das kleine Mädchen sondiert nach Hause entlassen werden. Eine Korrektur-Operation ist mit einem Gewicht von 5-7 Kilogramm Körpergewicht geplant.

Schlussfolgerung Komplexe urogenitale Fehlbildungen stellen prä- sowie postnatal eine große Herausforderung an die unterschiedlichsten medizinischen Disziplinen dar. Eine enge Kooperation und die Entbindung und Versorgung an einem Zentrum ist für das per se schwierige Management zur optimalen Betreuung betroffener Kinder unumgänglich.

\section{P 02.13 Das Kagami-Ogata Syndrom - eine wichtige Differentialdiagnose zum Beckwith-Wiedemann Syndrom. Ein Fallbericht}

Autoren Altmann $J^{1}$, Henrich $W^{1}$, Horn $D^{2}$, Korinth $D^{3}$, Verlohren $S^{1}$ Institut 1 Klinik für Geburtsmedizin; 2 Institut für Medizinische Genetik und Humangenetik; 3 Praxis für Humangenetik, Berlin/1 - 2 Charité -

Universitätsmedizin, Berlin

DOI 10.1055/s-0038-1670391

Patientin und Methode Eine 30-jährige I. Gravida wurde in 22 SSW mit Makrosomie, Omphalocele und Polyhydramnion, überwiesen. Es zeigte sich zusätzlich ein auffälliges Gesichtsprofil mit präfrontalem Ödem, eine geringe Magenfüllung sowie einer im Verlauf zunehmenden Organomegalie. Eine Makroglossie wurde nicht festgestellt. Bei V.a. auf Beckwith-Wiedemann-Syndrom wurde eine Amniozentese durchgeführt, die einen unauffälligen weiblichen Chromosomensatz ohne Mutation in der chromosomalen Region 11p15 ergab. Eine Mutation im CDKN1C-Gen wurde zudem ausgeschlossen. Im Verlauf wurden bei progredientem Polyhydramnion mehrere Entlastungspunktionen durchgeführt. In 35+5 SSW wurde die Sectio caesarea aufgrund von vorzeitigen Wehen durchgeführt und ein Mädchen mit $3660 \mathrm{~g}$ (>97. Perzentile) geboren. Postnatal wurde das Neugeborene mit einer respiratorischen Insuffizienz auf die Neonatologie aufgenommen. Es erhielt zunächst eine Atemunterstützung mit CPAP und im Anschluss eine Intubation mit einem Larynxtubus. In der Folge stabilisierte sich der Neonat respiratorisch.

Aufgrund der klinischen Charakteristika und dem bereits erfolgten Ausschluss eines Beckwith-Wiedemann Syndroms wurde der V.a. auf ein Kagami-Ogata Syndrom gestellt, welches durch eine uniparenterale paternale Disomie auf Chromosom 14 verursacht wird. Der genetische Befund zeigte untypischerweise eine Deletion von 336 kb der MEG3-Region auf Chromosom 14q32 des maternalen Allels. Bisher wurden wenige Fälle von Epimutationen und Mikro- deletionen auf dem maternalen Allel 14q32 veröffentlicht, welche zu einem Kagami-Ogata Syndrom führen.

Schlussfolgerung Bei Ultraschallzeichen eines Beckwith-Wiedemann Syndroms sollte differentialdiagnostisch ein Kagami-Ogata Syndrom in Betracht gezogen werden. Aufgrund der häufig vorkommenden respiratorischen Insuffizienz bei Kagami-Ogata Syndrom sollte die Geburt im Perinatalzentrum erfolgen.

\section{P 02.14 Case Report: Fetaler Mediastinaltumor}

Autoren Oelmeier de Murcia $\mathrm{K}^{1}$, Hammer K${ }^{1}$, Möllers $\mathrm{M}^{1}$, Braun J', Klockenbusch $\mathrm{W}^{1}$, Schmitz $\mathrm{R}^{1}$

Institut 1 Geburtshilfe, Universitätsklinik Münster

DOI 10.1055/s-0038-1670392

Zielsetzung Schilderung des Verlaufs bei fetalem Mediastinaltumor anhand eines Case Reports.

Methoden Aufnahme einer 22-jährigen G2P1 in der 35+1 SSW bei extern neu diagnostiziertem fetalem Mediastinaltumor $(4,5 \times 5,5 \times 5 \mathrm{~cm}$ im oberen vorderen Mediastinum gelegen) mit Verlagerung der Herzachse und des Ausflusstraktes/der Trachea sowie begleitendem Perikarderguss. Darüber hinaus unauffälliger, zeitgerecht entwickelter Fet.

Ergebnisse Im Verlauf Zunahme des fetalen Perikardergusses sowie neu aufgetretener Trikuspidalklappeninsuffizienz und deutlich dilatiertem Magen, so dass in der 36+1 SSW die Indikation zur primären Sectio gestellt wurde. Diese konnte komplikationslos erfolgen. Eine RDS-Prophylaxe war bereits in der 34 . SSW erfolgt. Postpartal Übergabe des Kindes an die Pädiater. $3000 \mathrm{~g}$, APGAR $6 / 8 / 8, \mathrm{pH} 7,32$. In der postpartalen Diagnostik konnte der $5 \mathrm{~cm}$ große zystische Mediastinaltumor bestätigt werden. Histopathologisch handelte es sich um ein reifes Teratom, das am dritten Lebenstag operativ entfernt werden konnte. Zwei Wochen nach Geburt konnte das Kind in gutem Allgemeinzustand in die ambulante Weiterbetreuung entlassen werden.

Zusammenfassung Ein optimales peripartales Management sowie die multidisziplinäre Zusammenarbeit zwischen Geburtshelfern, Neonatologen und Kinderkardiochirurgen sind entscheidend für die Versorgung von Feten mit Mediastinaltumor.

\section{P 02.15 Pränatale Darstellung einer Agenesie des Septum pellucidum als Hinweis auf eine „closed-lip“ Schizencephalie}

Autoren Bosselmann $S^{1}$, Kalle T von ${ }^{2}$, Mielke $G^{1}$

Institut 1 Pränatalzentrum Stuttgart; 2 Radiologisches Institut Olgahospital Klinikum Stuttgart

DOI 10.1055/s-0038-1670393

Patienten und Methode Eine 18-jährige IG/OP stellt sich mit 28+5 SSW zur weiterführenden Ultraschalldiagnostik bei Konsanguinität (Cousine - Cousin) vor. Eigen- und Familienanamnese sind unauffällig. Sonografisch ist das Septum pellucidum nicht darstellbar, die Länge des Corpus callosum liegt deutlich unter der 5. Perzentile, die Weite der Seitenventrikelhinterhörner ist grenzwertig (9,5 und 10,4 mm) und kardial fällt eine holosystolische Insuffizienz beider AV-Klappen bei milder Kardiomegalie auf. Die Nabelschur ist zystisch aufgetrieben und die Biometrie liegt im unteren Normbereich. Bei einer sonografischen Verlaufskontrolle mit 34+2 SSW ist ein kleiner subaortaler VSD nachweisbar. Eine MRT mit 29+6 SSW bestätigt die vorbeschriebenen ZNS-Befunde. Zusätzlich ist rechts-frontal ein Parenchymdefekt im Sinne einer „closedlip“ Schizencephalie darstellbar. Sonografisch lässt sich diese auch in Kenntnis des MRT-Befundes nicht abgrenzen (Verlaufskontrolle 34+2 SSW). Die pränatal erhobenen Befunde werden postnatal bestätigt.

Schlussfolgerung In der pränatalen Sonografie können Auffälligkeiten im Bereich der Mittellinie des ZNS als Hinweis auf schwere Hirnentwicklungsstörungen vorliegen. Das Cavum Septi Pellucidi (CSP) gehört zu diesen Strukturen und wird als Leitstruktur zur Einstellung der korrekten Ebene für die Kopfbiometrie dargestellt. Zu den häufigeren Auffälligkeiten in diesem Bereich gehört 
das Fehlen des CSP als möglicher Hinweis auf eine Corpus-Callosum-Agenesie (ACC). Ein dilatiertes CSP ist als Marker für Chromosomenstörungen zu werten (z. B. Trisomie 18). Das Fehlen der beiden Blätter des Septum pellucidum als laterale Begrenzung des CSP ist mit einer Inzidenz 2-3/100 000 in der Gesamtbevölkerung ein sehr seltener Befund. Differentialdiagnostisch sollte hierbei vor allem an eine Holoprosencephalie, an eine Septo-Optische-Dysplasie oder eine ACC gedacht werden. Seltener kommen eine Schizencephalie (1/70 000 Lebendgeburten), eine Ruptur des Septum pellucidum bei Hydrocephalie oder die isolierte Agenesie des Septum pellucidum in Betracht. Die Diagnose insbesondere einer „closed-lip“ - Schizencephalie wird pränatal äußerst selten gestellt. Der Fall demonstriert einerseits die Bedeutung von Anomalien des CSP als einfach darzustellender Hinweis auf Hirnfehlbildungen. Zusätzlich zeigt dieser Fall die ergänzenden Möglichkeiten der MRT in der pränatalen Abklärung von ZNS-Auffälligkeiten.

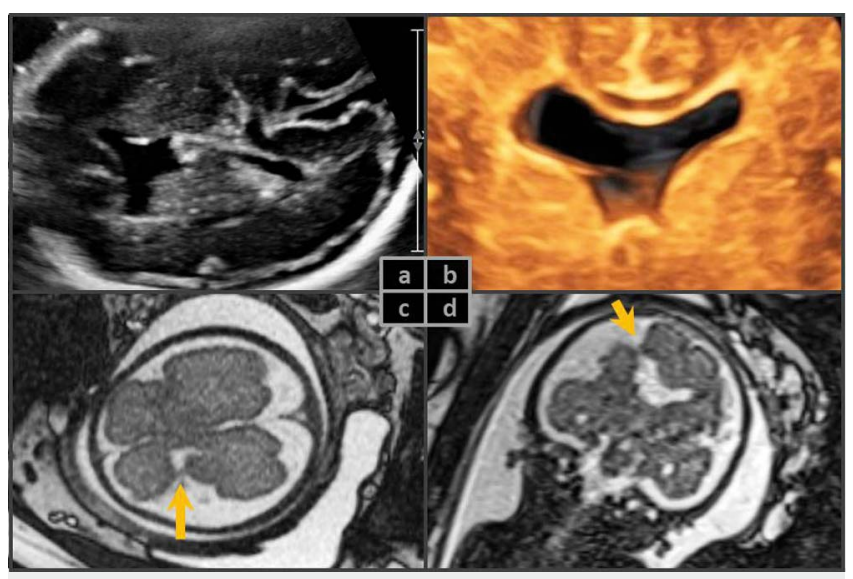

Abb. 1

\section{P 02.16 Postnatale Diagnose eines familiären Nijmegen-Breakage-Syndrom - pränatale Mikrozephalie/Ventrikulomegalie als Ausdruck einer syndromalen Erkrankung mit schwieriger Beratungssituation}

Autoren Beyer $J^{1}$, Voigt $C^{1}$, Fröber $R^{2}$, Schreyer $I^{3}$, Weise $A^{4}$, Schleußner $E^{1}$, Schneider $\mathrm{U}^{1}$

Institut 1 Klinik für Geburtsmedizin; 2 Institut für Anatomie/Institut für Rechtsmedizin; 3 Institut für Praxis für Humangenetik (ZAM); 4 Institut für Humangenetik/ 1 - 4 Universitätsklinikum Jena

DOI 10.1055/s-0038-1670394

Einführung Die Beratung von Paaren bei isolierten Hirnfehlbildungen ist individuell schwierig aufgrund der unklaren Prognose. Die Fortschritte in der Humangenetik tragen inzwischen zur Klärung der Ursachen vieler fetaler Fehlbildungen bei, die noch vor wenigen Jahren nicht diagnostiziert werden konnten. Die schwierige Beratungssituation wird anhand eines komplexen Falles demonstriert.

Kasuistik 32-jährige III Gravida, I Para. 2008 induzierter Spätabort bei fetaler Schizenzephalie „open lips“, damalige genetische Diagnostik ohne auffälliges Ergebnis. 2009 Geburt einer gesunden Tochter. 3. Schwangerschaft, 18+4 SSW: fetale Mikrozephalie, Ventrikulomegalie von 15 mm bds., Retrognathie. Ammniocentese: unauffälliges Karyogramm. Bei persistierender Mikrozephalie Abortinduktion bei 20+6 SSW. wegen dringendem V.a. autosomal rezessive syndromale Erkrankung mit schlechter Prognose. Bei unklarer endgültiger Diagnose extrem schwierige Beratungssituation und hohe Belastung für das Paar. Nach 4 Wochen schließlich Diagnosestellung eines Nijmegen-BreagakeSyndroms (NBS) mittels PANEL-Diagnostik. Bei den Eltern wird der Überträgerstatus nachgewiesen, an asservierter DNA des ersten Kindes kann auch ein NBS belegt werden.
Nijmegen-Breakage-Syndrom (NBS) Das NBS ist ein ChromosomenbruchSyndrom (Ataxia-teleangiektasie-Variante) mit unterschiedlichen Hirn- \& Gesichtsfehlbildungen, geistiger Retardierung, Wachstumsverzögerung, Immundefizit und besonders hoher Malignomneigung mit sehr schlechter Prognose. Es tritt mit einer Häufigkeit von etwa 1:100000 auf, Betroffene erreichen nur selten das Erwachsenenalter. Eine extrem hohe Strahlensensibiltät ist beschrieben, auch für heterozygote Mutationsträger ist ein erhöhtes Karzinomrisiko, besonders nach Strahlenbelastung beschrieben. Das Wiederholungsrisiko beträgt 25\% für Folgeschwangerschaften.

Schlussfolgerung Die Beratung bei isolierter fetaler Hirnfehlbildung bleibt aufgrund der oft unklaren Prognose eine Herausforderung. Durch die Fortschritte in der humangenetischen Diagnostik können inzwischen die Ursachen von Fehlbildungen auch noch nach mehreren Jahren geklärt werden. Die Diagnose des NBS ist auch für die Überträger relevant, eine unnötige Strahlenbelastung sollte vermieden werden, ein Monitoring für Karzinome kann angeboten werden.

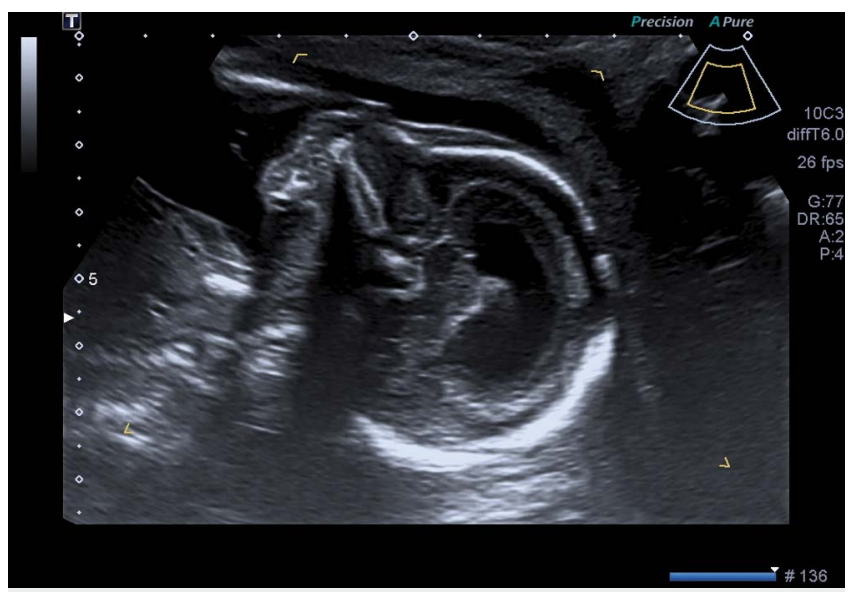

Abb. 1

\section{Wissenschaftliche Vortragssitzungen}

Wi-Vo 01 MSK
Do. 15.11. 08:30 - 10:00 Shanghai 3

\section{01.01 Präoperativer kontrastverstärkter Ultraschall (CEUS) als Prädiktor für funktionelles Ergebnis und Reruptur bei Patienten mit Supraspinatussehnenruptur}

Autoren Fischer $\mathrm{C}^{1}$, Mick $\mathrm{P}^{1}$, Weber $\mathrm{MA}^{2}$, Zeifang $\mathrm{F}^{3}$, Schmidmaier $\mathrm{G}^{1}$, Kunz $\mathrm{P}^{4}$

Institut 1 Zentrum für Orthopädie, Unfallchirurgie und Paraplegiologie, HTRG - Heidelberg Trauma Research Group, Universitätsklinikum Heidelberg;

2 Institut für diagnostische und interventionelle Radiologie,

Universitätsmedizin Rostock; 3 Ethianum Klinik Heidelberg; 4 Klinik für Schulter- und Ellenbogenchirurgie, Katholisches Klinikum Mainz DOI 10.1055/s-0038-1670395

Einführung Die Indikation zur Rekonstruktion einer Supraspinatus (SSP)-Sehnenläsion erfolgt wesentlich auf Grundlage der Verfettung des SSP-Muskelbauches sowie der Sehnenretraktion in der Magnetresonanztomografie (MRT). Diese morphologischen Parameter erlauben jedoch nur eingeschränkt Rückschluss auf das funktionelle Ergebnis sowie eine mögliche Reruptur nach Sehnennaht. Bis heute fehlen funktionelle, präoperative Surrogatmarker zur Unterstützung der Therapieentscheidung bei Patienten mit SSP-Sehnenläsion. 
Der kontrastverstärkte Ultraschall (CEUS) ermöglicht eine funktionelle, dynamische Evaluation der SSP-Muskelperfusion und -vitälität. Ziel der Studie war die Evaluation der präoperativen SSP-Perfusion und deren Zusammenhang mit der postoperativen Schulterfunktion sowie der Rerupturrate der SSP-Sehne.

Material und Methoden 72 Patienten mit isolierter SSP-Läsion wurden prospektiv in die Studie eingeschlossen. Unmittelbar vor sowie sechs Monate nach der Operation wurden klinische Funktionsscores erhoben sowie ein MRT angefertigt. Zudem erfolgte jeweils ein CEUS des SSP- sowie des Trapeziusmuskels der operierten sowie der gesunden Gegenseite. Mittels einer spezifischen Quantifizierungssoftware $\left(V^{2}\right.$ ebox ${ }^{\circledR}$ ) wurden Perfusionsparameter wie Peak Enhancement (PE), Wash-in Perfusion Index (WiPI) und Rise Time (RT) bestimmt. Präoperative Perfusionsparameter (Ratio SSP/Trapezius) wurden mit postoperativen Funktionsscores sowie der postoperativen morphologischen Sehnenintegrität (MRT und Sono) korreliert.

Resultate 55 Patienten konnten nachuntersucht werden. Patienten mit geringer präoperativer Perfusionsratio zwischen SSP und Trapezius wiesen sechs Monate postoperativ eine signifikant schlechtere klinische Funktion $(p=0,002)$ sowie eine signifikant höhere Rerupturrate $(p=0,001)$ auf als solche mit hoher Perfusionratio zwischen SSP und Trapezius. Alle Patienten mit einer präoperativen SSP/Trapezius-Perfusionsratio innerhalb des ersten Tertils zeigten sechs Monate postoperativ eine Reruptur.

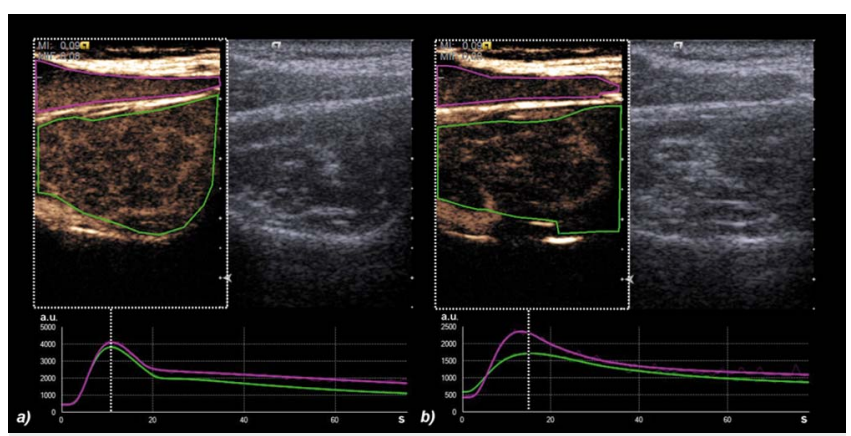

Abb. 1

Schlussfolgerung Die Perfusionsratio von SSP/Trapezius im CEUS korreliert mit der postoperativen Funktion und erlaubt die Prädiktion einer Reruptur nach Sehnenrekonstruktion bei Patienten mit SSP-Sehnenläsion. Als Prädiktor für funktionelles Ergebnis und Reruptur könnte die präoperative CEUS- Quantifizierung ein wertvoller Baustein für die Therapieentscheidung bei Patienten mit SSP-Sehnenläsionen darstellen.

\section{01.02 Prospective Evaluation of Changes in Elastic Properties of Ruptured Achilles Tendons by Shear Wave Elastography}

Authors Henssler $\mathrm{L}^{1}$, Miksch $\mathrm{M}^{1}$, Weber $\mathrm{J}^{1}$, da Silva $\mathrm{NP}^{2}$, Jung $\mathrm{EM}^{2}$,

Docheva $D^{1}$, Nerlich $M^{1}$, Pfeifer $C^{1}$, Frankewycz $B^{1,3}$

Institute 1 Department of Trauma Surgery; 2 Department of Radiology;

3 Sibley School of Mechanical and Aerospace Engineering, Cornell University, New York/1 - 2 Regensburg University Medical Center

DOI 10.1055/s-0038-1670396

Purpose Biomechanical properties of tendon change after rupture. Shear wave elastography (SWE) allows evaluating biomechanical properties ('elastografic stiffness') of soft tissue. The main objective of this study was to show the development of changes in elastic properties of the Achilles tendon (AT) in the early healing phase after rupture. Further objectives were to examine a correlation between the patients' subjective outcome and changes in the elastic properties and to compare non-operatively vs. operatively treated tendons.

Methods SWE was performed on ATs of patients who had ruptured their AT and received operative $(\mathrm{O})$ or non-operative $(\mathrm{N})$ treatment at stated intervals $(1,3,6,9$ and 12 weeks after injury). Patients were included who presented at our emergency department with an isolated partial or total rupture of the AT. Exclusion criteria were bi-lateral injury in their history, arthrodesis of one of the upper ankle joints, neuropathic or malignant diseases, age $<18$ years. Both AT (injured and healthy) of each patient were scanned in the distal, middle and proximal portion as well as in the rupture area (of the injured tendon respectively) using Virtual TouchTM IQ sonography (Siemens Acuson S3000, 4-9 MHz linear probe). For evaluation of the patients' outcome two established functional scores were used. Statistical analysis was performed by using linear regression analysis for the different time points and paired t-test for independent samples.

Results 12 patients were included in the study by the time of abstract submission [9 $(\mathrm{N}), 3(\mathrm{O})$ ]. Results indicate a positive linear regression between shear wave velocity at the rupture site and time after injury [R2 $=0.697$, $\mathrm{p}<0.001]$. A significant difference in stiffness was found between $\mathrm{O}$ and $\mathrm{N}$ after 3 weeks at the rupture site $[p=0.025]$. 6 weeks after injury the rupture site gains values equally to the contralateral tendon. No significant correlation was found between SWE and functional outcome.

Conclusion Quantitative SWE is capable of displaying changes in elastic properties during tendon healing. Furthermore, a noticeable increase of elastografic stiffness especially between 3 and 6 weeks after injury was shown after an initial absence of elastografic stiffness in the first weeks after injury. Further analyses need to be done in order to correlate ultrasound findings with clinical scores and biomechanical parameters. To assess the utility of SWE for monitoring purposes more studies are necessary.

\section{Rupture site}

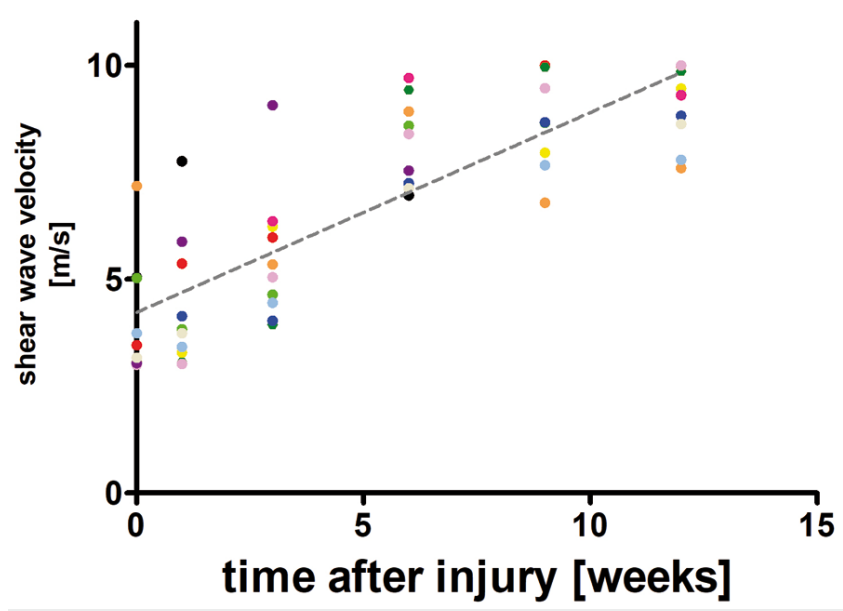

Fig. 1 Positive linear regression between shear wave velocity at the rupture site and time after injury [R2 $=0.697, p<0.001]$

\section{01.03 Kontrastmittelverstärkter Ultraschall (CEUS) zur Beurteilung der tibialen Knochenperfusion bei physiologischer und gestörter Frakturheilung mit Pseudarthrosenbildung}

Autoren Fischer $\mathrm{C}^{1}$, Haug $\mathrm{T}^{1}$, Weber $\mathrm{MA}^{2}$, Kauczor $\mathrm{HU}^{3}$, Bruckner $\mathrm{T}^{4}$, Schmidmaier $\mathrm{G}^{1}$

Institut 1 Zentrum für Orthopädie, Unfallchirurgie und Paraplegiologie, HTRG - Heidelberg Trauma Research Group; 2 Institut für diagnostische und interventionelle Radiologie, Universitätsmedizin Rostock; 3 Diagnostische und interventionelle Radiologie; 4 Medizinische Biometrie und Informatik, Universität Heidelberg/ 1,3 Universitätsklinikum Heidelberg DOI 10.1055/s-0038-1670397

Einführung Diese Studie untersuchte den Nutzen des CEUS für die Beurteilung verschiedener Perfusionsmuster nach Tibiafrakturen, die zur Ausheilung oder Pseudarthrosenbildung führen können. 
Material und Methoden Von 2014 bis 2017 wurde bei 107 Patienten mit Tibiafraktur oder Tibia-Pseudarthrose eine CEUS-Untersuchung durchgeführt. Die CEUS-Untersuchung fand zum regulären 26-Wochen Nachuntersuchungstermin statt, bei Patienten mit Pseudarthrose unmittelbar vor der Revisionsoperation. Es wurden Zeit-Intensitäts-Kurven der Kontrastmittelanflutung im Frakturspalt erstellt und quantitative Parameter wie "Wash-in Rate“ (WiR), „Peak Enhancement“ (PE) sowie „Wash-in Perfusion Index“ (WiPI) berechnet. Resultate 34 Patienten wurden eingeschlossen, davon 14 verheilte Frakturen, 12 aseptische Pseudarthrosen und 8 Infektpseudarthrosen. Die Perfusionsparameter WiR, PE and WiPI zeigten bei aseptischen Pseudarthrosen signifikant niedrigere Werte als bei verheilten Frakturen ( $p=0,009 ; 0,009 ; 0,012$ ). Im Gegensatz dazu waren WiR, PE and WiPI bei Infektpseudarthrosen höher als bei physiologisch verheilten Frakturen ( $p=0,034 ; 0,056 ; 0,029)$.
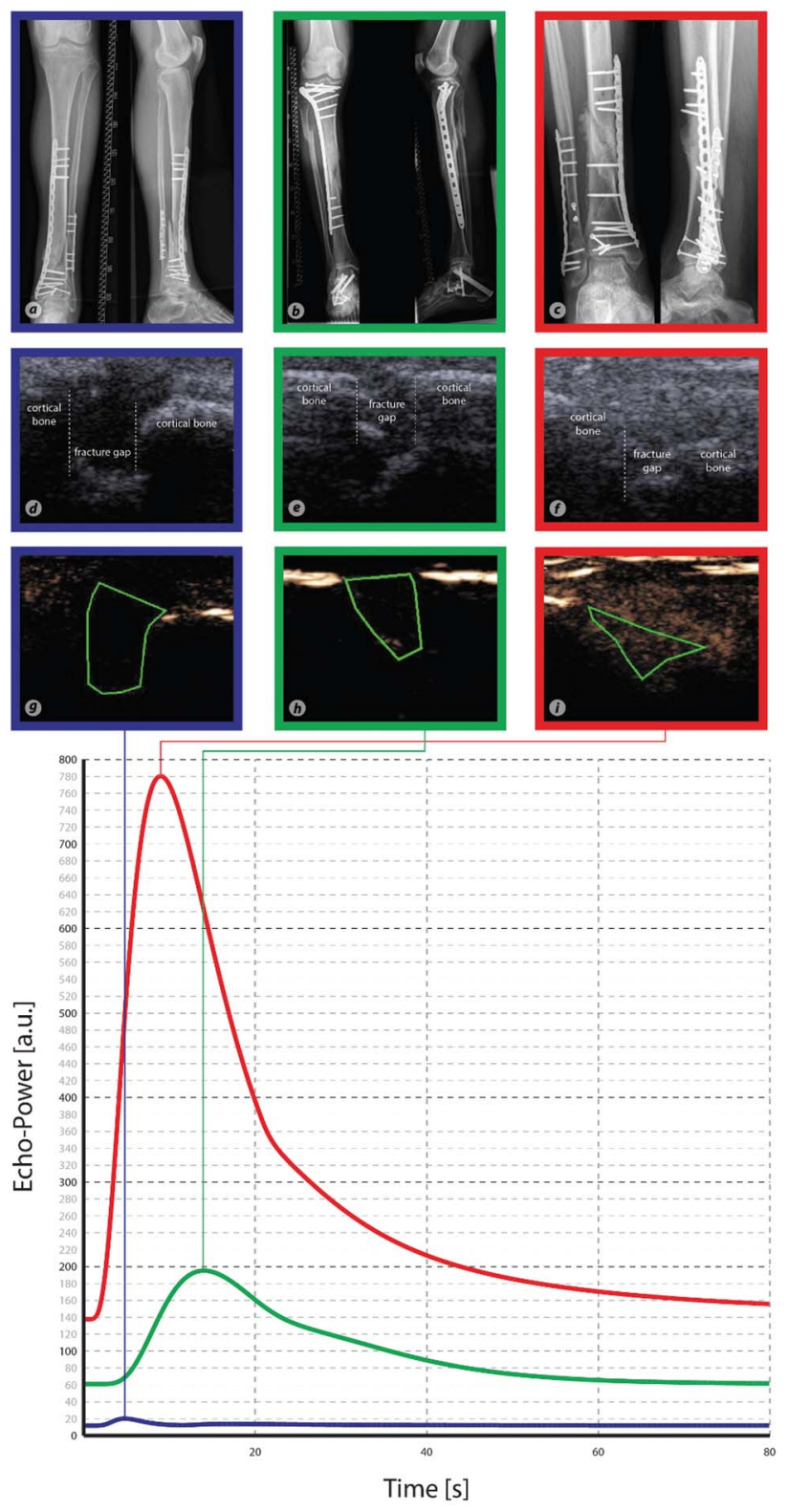

Abb. 1

Schlussfolgerung CEUS ist eine geeignete Methode zur Beurteilung der ossären Perfusion nach Fraktur. Im CEUS zeigen sich Perfusionsunterschiede von Tibiafrakturen mit regulärem Heilungsverlauf und solchen, die eine Pseudarthrose ausgebildet haben. Abweichungen von der physiologischen ossären Per- fusion nach Tibiafraktur scheinen mit der Ausbildung einer Pseudarthrose zusammenzuhängen.

\section{01.04 Kontrastverstärkter Ultraschall (CEUS) und Elastografie zur Beurteilung der Deltoideus Integrität nach inverser Schulterprothese}

Autoren Fischer $C^{1}$, Krammer $D^{1}$, Hug $A^{2}$, Weber $M A^{3}$, Kauczor $\mathrm{HU}^{4}$, Krix $\mathrm{M}^{3}$, Bruckner $\mathrm{T}^{5}$, Kunz $\mathrm{P}^{1}$, Schmidmaier $\mathrm{G}^{1}$, Zeifang $\mathrm{F}^{1}$

Institut 1 Zentrum für Orthopädie, Unfallchirurgie und Paraplegiologie, HTRG - Heidelberg Trauma Research Group; 2 Klinik für Paraplegiologie; 3 Institut für diagnostische und interventionelle Radiologie, Universitätsmedizin,

Rostock; 4 Diagnostische und interventionelle Radiologie; 5 Medizinische Biometrie und Informatik, Universität Heidelberg/ 1,2,4 Universitätsklinikum Heidelberg

DOI 10.1055/s-0038-1670398

Einführung Das Operationsergebnis nach Implantation einer inversen Schulterprothese hängt maßgeblich vom Zustand des Musculus deltoideus ab, welcher in dieser Studie mit neuen Ultraschallmodalitäten und Elektromyografie (EMG) untersucht wurde. Kontrastverstärkter dynamischer Ultraschall (contrast-enhanced ultrasound - CEUS) und Elastografie (acoustic radiation force impulse - ARFI) wurden eingesetzt um die Perfusion und Elastizität des Deltamuskels zu erfassen. Diese wurden mit funktionellen und klinischen Ergebnissen verglichen, um so ein umfassenderes Bild der Integrität des Deltamuskels zu erhalten.

Methoden 64 Patienten (mittleres Alter: 72,9 Jahre), welche zwischen 2004 und 2013 eine inverse Schulterprothese erhielten, wurden beidseits standardisiert mit Ultraschall und EMG untersucht. Als funktionelle Score wurden der Constant Score und der American Shoulder and Elbow Surgeons Standardized Shoulder Assessment Form score erhoben. Die CEUS-Perfusionsparameter Wash-in Perfusion Index (WiPI), Time to Peak (TTP) und Rise Time (RT) der operierten Seite und der Gegenseite wurden miteinander verglichen, ebenso wie die Deltaperfusion von Patienten mit überdurchschnittlichem und unterdurchschnittlichem funktionellem Ergebnis. Die Elastizität des Deltamuskels wurde durch ARFI erhoben.

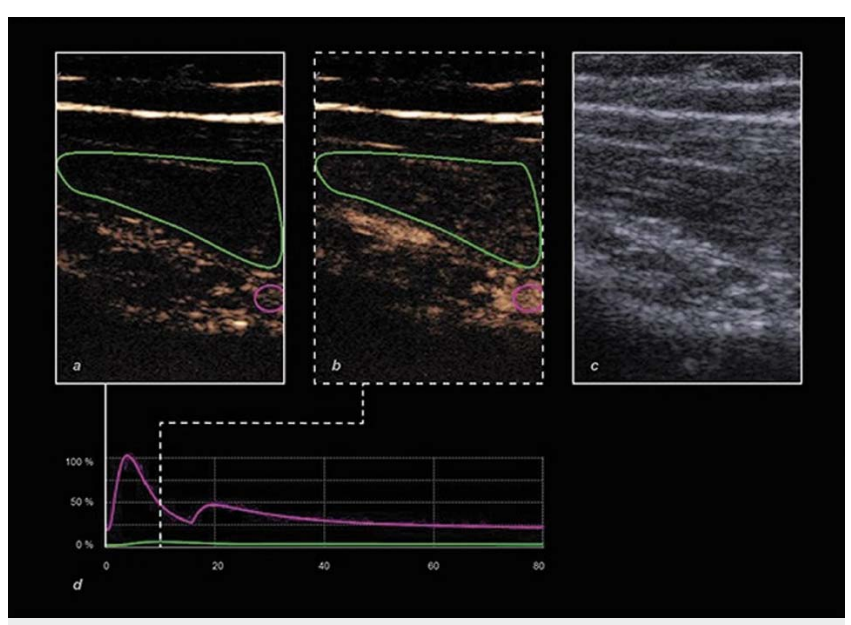

Abb. 1

Resultate Nach Versorgung mit einer inversen Prothese zeigte sich die Durchblutung des Deltamuskels (WiPI: $\Delta=-12 \% \pm 22 \%, p=0,001$ ) und die Funktion der Schulter (Constant Score: $\Delta=-14 \pm 24, p=0,0001$ ) beeinträchtigt im Vergleich zur Gegenseite. Dieses Perfusionsdefizit stand in Zusammenhang mit einem eingeschränkten Bewegungsausmaß (TTP und Anteversion: $r=-0,29$, $p=0,022$ ). Bei Patienten mit überdurchschnittlichem funktionellem Resultat zeigte sich eine höhere Perfusion (RT: $\Delta=-33 \% \pm 13 \%, \mathrm{p}=0,038$ ). Eine tendenziell höhere Gewebssteifigkeit konnte auf der betroffenen Seite erhoben werden (ARFI: $\Delta=0,2 \pm 0,9 \mathrm{~m} / \mathrm{s}, \quad p=0,0545$ ). Mittels EMG konnten funk- 
tionsrelevante Läsionen des Nervus axillaris ausgeschlossen werden. Die Ergebnisse waren unabhängig vom Zeitintervall nach OP.

Schlussfolgerung CEUS zeigte eine verminderte durchschnittliche Durchblutung des Deltamuskels der operierten Seite. Geringere Perfusion korrelierte mit einer schlechteren Schulterbeweglichkeit. CEUS könnte in Zukunft als Surrogatparameter für die muskuläre Integrität dienen und funktionelle Einschränkungen der Schulter nach inverser Prothese prognostizieren.

\section{01.05 Inter-Rater Reliability of an Ultrasound Protocol to Evaluate the Anterolateral Ligament of the Knee}

Authors Kandel $\mathrm{M}^{1,2}$, Cattrysse $\mathrm{E}^{3}$, De Maeseneer $\mathrm{M}^{4}$, Lenchik $\mathrm{L}^{5}$, Paantjens $\mathrm{MA}^{2,6}$, Leeuw $\mathrm{M}^{2,6}$

Institute 1 Physial Therapy Practice M. Kandel, Oberriet, Switzerland; 2 SOMT University, Amersfoort, The Netherlands; 3 Department of Experimental Anatomy, VUB Brussels, Belgium; 4 Department of Radiology, UZ Brussel, Belgium; 5 Radiologic Sciences-Radiology, Wake Forest Baptist Medical Center, North Carolina, USA; 6 Ministerie van Defensie, The Netherlands DOI 10.1055/s-0038-1670399

Objectives The aim of this study was to conduct a valid and reliable musculoskeletal ultrasound (MSK US) protocol to evaluate the anterolateral ligament (ALL) of the knee in clinical practice. Imaging assessments to evaluate the integrity of the ALL are clinically relevant in patients with suspected tear of the anterior cruciate ligament (ACL) and clinical signs of anterolateral instability. MSK US is a non-invasive, cost-effective, static, and dynamic method to visualize the ALL in real time. There is, however, no consensus how to reliably visualize the ALL with MSK US in clinical practice.

Methods A Thiel technique cadaveric specimen was used to validate the optimal scanning position and create an MSK US protocol to evaluate the ALL of the knee. Three musculoskeletal sonographers acquired short- and long-axis images of 34 knees from 18 healthy participants. Besides clinical evaluation, ALL length, -thickness, -width, and distance between ALL insertion and the tibia plateau were measured. To evaluate reliability the Intraclass Correlation Coefficient (ICC) was used.

Results The inter-rater reliability for ALL thickness was poor, ICC 0.35 (95\%Cl: $-0.06-0.63)$. The inter-rater reliability for ALL length and width was good, ICC $0.80(95 \% \mathrm{Cl} 0.64-0.89)$, resp. ICC $0.88(95 \% \mathrm{Cl} 0.79-0.94)$ and the interrater reliability for the distance between ALL insertion and tibia plateau was excellent, ICC 0.96 ( $95 \% \mathrm{Cl} 0.93-0.98)$.

Conclusions The protocol presented in this study is a valid and reliable tool to evaluate the anterolateral ligament of the knee. There is an excellent reliability for the distal part of the ALL. As injuries almost always occur in this part of the ligament, this protocol may become a useful tool to evaluate the ALL in patients with suspected $\mathrm{ACL}$ tears in clinical practice.

Keywords anterolateral ligament; musculoskeletal ultrasound; knee instability; anterior cruciate ligament; diagnostic imaging.

\section{01.06 Delta-split vs. deltoideopektoraler Zugang zur Plattenosteosynthese des proximalen Humerus - Evaluation mittels CEUS}

\footnotetext{
Autoren Fischer $\mathrm{C}^{1}$, Frank $\mathrm{M}^{1}$, Kunz $\mathrm{P}^{2}$, Tanner $\mathrm{M}^{1}$, Weber $\mathrm{MA}^{3}$, Mogghadam $A^{4}$, Schmidmaier $G^{1}$, Hug $A^{5}$

Institut 1 Zentrum für Orthopädie, Unfallchirurgie und Paraplegiologie;

2 Zentrum für Orthopädie und Unfallchirurgie, Katholisches Klinikum Mainz;

3 Diagnostische und Interventionelle Radiologie; 4 Zentrum für

Unfallchirurgie, Orthopädie und Sportmedizin, Aschaffenburg-Alzenau;

5 Zentrum für Paraplegiologie/ 1,3,5 Universitätsklinikum Heidelberg DOI 10.1055/s-0038-1670400
}

Einleitung Die Wahl der minimalinvasiven Delta-Split-Methode (MIPO) anstatt der konventionell offenen Plattenosteosynthese mittels deltoideopektoralem Zugang (ORIF) zur Versorgung proximaler Humerusfrakturen wird in der Lite- ratur kontrovers diskutiert. Dabei werden einer weitgehenden Gewebeschonung des MIPO-Zugangs Läsionen des N. axillaris und des M. deltoideus gegenübergestellt. In dieser Studie wurde die Vitalität des M. deltoideus nach MIPO vs. ORIF durch Messung der muskulären Mikroperfusion mittels kontrastverstärktem Ultraschall (CEUS) verglichen und mit der Schulterfunktion sowie der $\mathrm{N}$. axillaris Integrität korreliert.

Material und Methoden Es wurden 50 Patienten (30 ORIF vs. 20 MIPO), die zwischen 2011 und 2014 eine Plattenosteosynthese des proximalen Humerus erhielten, postoperativ untersucht. CEUS und Dopplersonografie wurden nach spezifischer Beübung des M. deltoideus beidseits durchgeführt um die Ergebnisse der operierten Schulter intraindividuell mit der gesunden Gegenseite zu vergleichen. Die klinische Schulterfunktion, Patientenzufriedenheit sowie das psychosoziale Ergebnis wurden mittels etablierter Scores evaluiert (Constant, DASH, Simple Shoulder Test, ASES, SF-12). Der M. deltoideus wurde elektromyografisch auf eine Läsion des N. axillaris untersucht.

Resultate Keine der Untersuchungsmodalitäten wies signifikante Unterschiede zwischen den beiden Operationszugängen auf. Die Mikroperfusion des M. deltoideus im CEUS war in beiden Gruppen gleich ausgeprägt, jedoch jeweils signifikant schwächer als auf der gesunden Gegenseite. Diese Resultate entsprachen den Ergebnissen der funktionellen Schulterbewertung (Constantund ASES-Score $p \leq 0,001$ für ORIF und MIPO). Keiner der versorgten Patienten wies EMG-relevante Axillarisschäden auf. Auch in Bezug auf Schmerz, Bewegungen des alltäglichen Lebens, Bewegungsumfang oder Kraft wurde kein Unterschied zwischen ORIF und MIPO festgestellt.

Schlussfolgerung In dieser Studie konnte mittels CEUS gezeigt werden, dass die Wahl des Operationszugangs (ORIF vs. MIPO) keinen Einfluss auf die Perfusion und Funktion des M. deltoideus hatte. Das funktionelle, sonografische und neurologische Ergebnis war in beiden Patientengruppen gleich. Die Entscheidung des operativen Zugangs zur plattenosteosynthetischen Versorgung von proximalen Humerusfrakturen sollte somit in erster Linie von der Patientenkonstitution, der Frakturmorphologie sowie der Erfahrung und Präferenz des Operateurs abhängig gemacht werden.

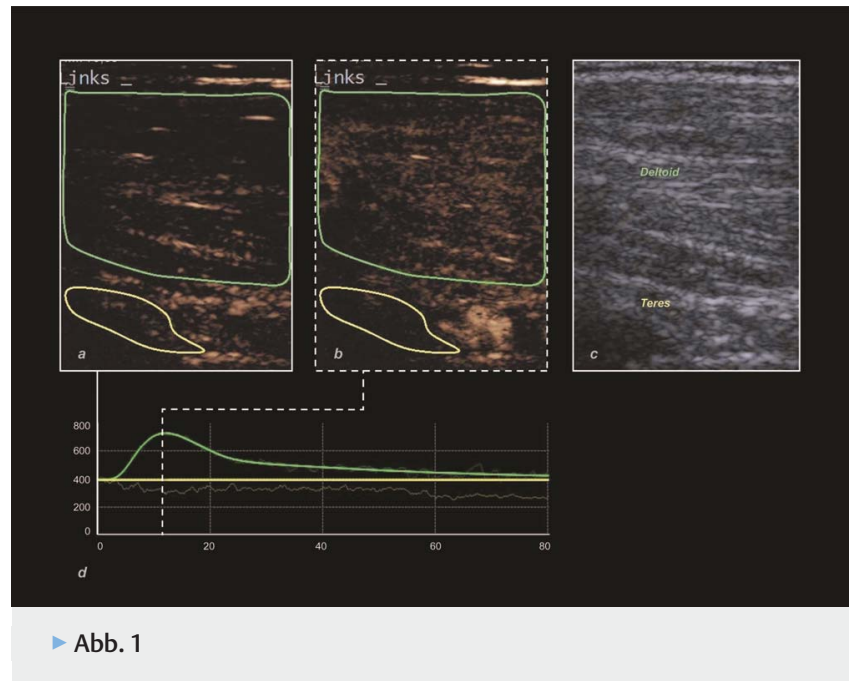

\section{01.07 Hohe Intra- und Interobserver Reliabilität kontrastverstärkter Ultraschallergebnisse (CEUS) im Assessment der Deltamuskelperfusion an gesunden Probanden im Untersucher- und Gerätevergleich}

\footnotetext{
Autoren Kiesl $\mathrm{S}^{1}$, Weber $\mathrm{MA}^{2}$, Kunz $\mathrm{P}^{3}$, Fischer $\mathrm{C}^{4}$

Institut 1 Zentrum für Orthopädie, Unfallchirurgie und Paraplegiologie, HTRG - Heidelberg Trauma Research Group; 2 Institut für diagnostische und interventionelle Radiologie, Universitätsmedizin Rostock; 3 Diagnostische und
} 
interventionelle Radiologie; 4 Medizinische Biometrie und Informatik, Universität Heidelberg/1,3 Universitätsklinikum Heidelberg DOI 10.1055/s-0038-1670401

Einführung Überprüfung der Reproduzierbarkeit muskuloskelettaler kontrastverstärkter Ultraschallergebnisse (CEUS) von zwei verschiedenen Untersuchern an zwei High-End-Geräten unterschiedlicher Hersteller als Basis für multizentrische Studien.

Material und Methoden Es wurden 21 gesunde Probanden ohne jegliche Schulterprobleme in der Vorgeschichte (9 Frauen, 12 Männer; mittleres Alter 26,5 $\pm 5,6$ Jahre) rekrutiert. Die konventionelle Ultraschalluntersuchung und CEUS Quantifizierung des Deltamuskels wurden jeweils identisch und standardisiert von zwei Untersuchern an zwei High-End-Geräten (Siemens ACUSON S3000, Erlangen, Germany und Philips Epiq 7, Hamburg, Germany) durchgeführt. Das dreiteilige Studienprotokoll beinhaltete 166 Ultraschalluntersuchungen, um jeweils die Intra- und Interobserver-Reproduzierbarkeit und die Vergleichbarkeit der zwei Ultraschallsysteme zu überprüfen. Vor jeder CEUS-Untersuchung beübten die Probanden den Deltamuskel standardmäßig. Nach der Bolusinjektion von 2,4 ml SonoVue Kontrastmittel, begann die Aufnahme eines 90-sekündigen Videoclips, um die Deltamuskelperfusion zu visualisieren. Alle Videoclips wurden mit der VueBox Software (Bracco Imaging, Mailand, Italien) quantifiziert und der Wash-in Perfusion Index (WiPI) Perfusionsparameter wurde generiert und statistisch ausgewertet. Hierfür wurden die Spearman Korrelation (r), t-Tests und lineare Regressionsmodelle (R) mithilfe von IBM SPSS 25,0 in Kooperation mit dem Heidelberger Institut für Medizinische Biometrie berechnet.

Resultate Es wurde eine hohe Intraobserver-Reproduzierbarkeit der Deltaperfusionsmessergebnisse erreicht, wenn derselbe Untersucher die Sonografie durchführte $(r=0,91 ; R=0,94)$. Die Interobserver-Reproduzierbarkeit der Deltaperfusionsmessergebnisse bei zwei verschiedenen Untersuchern war ebenfalls hoch ( $r=0,78 ; R=0,77)$. Der Vergleich der zwei High-End-Geräte ergab eine niedrigere, aber stets hohe Übereinstimmung $(r=0,71 ; R=0,74)$. Alle drei Untersuchungsschritte ergaben keine signifikanten Unterschiede im tTest $(p=0,56 ; 0,58 ; 0,30)$.

Schlussfolgerung Die hohe Intra- und Interobserver Reproduzierbarkeit der Messergebnisse der Deltaperfusion stellen eine Basis für zukünftige klinische Studien mit muskuloskelettalem CEUS dar, solange das Studienprotokoll befolgt wird. Multizentrische, Geräte-unabhängige Studien könnten folglich in der Lage sein, inter-individuelle Unterschiede der muskuloskelettalen CEUS Messergebnisse zu vergleichen und zu untersuchen.

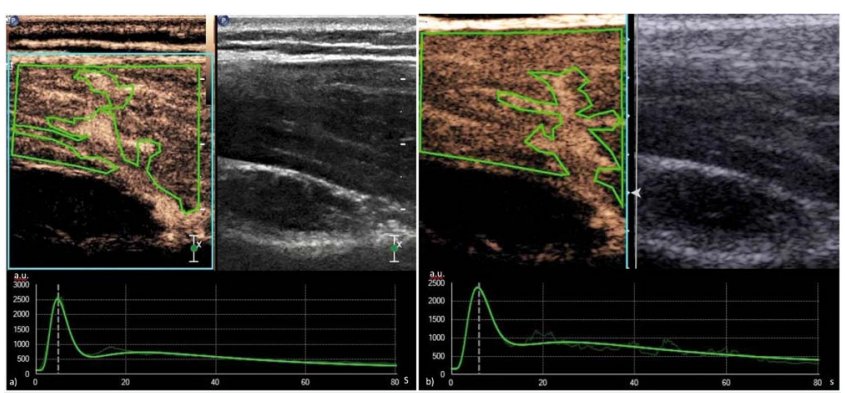

Abb. 1
Wi-Vo 02 Gynäkologie/Geburtshilfe I

Do. 15.11. 08:30 - 10:00 Boston 3

\section{02.01 Gehirnentwicklung bei Feten von diabetischen und gesunden Müttern im Vergleich}

Autoren Hammer $\mathrm{K}^{1}$, Gründahl FR², Braun J', Oelmeier de Murcia $\mathrm{K}^{1}$, Köster $\mathrm{HA}^{1}$, Möllers $\mathrm{M}^{1}$, Steinhard ${ }^{3}$, Klockenbusch $\mathrm{W}^{1}$, Schmitz $\mathrm{R}^{1}$ Institut 1 Abteilung für Gynäkologie und Geburtshilfe, Universitätsklinikum Münster; 2 Abteilung für Gynäkologie und Geburtshilfe, St. Franziskus-

Hospital Münster; 3 Department für fetale Kardiologie, Herz- und Diabeteszentrum Nordrhein-Westfalen, Bad Oeynhausen DOI 10.1055/s-0038-1670402

Ziel Ziel der Studie war es, Gehirnstrukturen bei Feten von diabetischen und gesunden Schwangeren im zweiten und dritten Trimester zu vergleichen.

Methoden und Material In einer retrospektiven Untersuchung wurden der Kopfumfang, der Kleinhirndurchmesser, die Größe der Zisterna magna, des Cavum septi pellucidi (CSP) und des Seitenventrikels in archivierten Ultraschallbildern von Feten zwischen der 20. und 41. Schwangerschaftswoche gemessen. Die Werte von 231 Feten diabetischer Mütter wurden mit den Werten von 231 Feten gesunder Schwangerer des entsprechenden Gestationsalters verglichen. Die Diabetesgruppe wurde hierbei in drei Untergruppen unterteilt: vorbestehender maternaler Diabetes, diätetisch eingestellter Schwangerschaftsdiabetes und insulinabhängiger Schwangerschaftsdiabetes. Ergebnisse Die durchschnittliche Weite des CSP und des Seitenventrikels war in der Diabetesgruppe größer als in der Kontrollgruppe (für beide Parameter $\mathrm{P}<0,001)$. Für die anderen Parameter konnte kein Unterschied festgestellt werden. Die Ergebnisse waren in den drei Subgruppen einheitlich.

Schlussfolgerung Maternaler Diabetes scheint mit strukturellen Veränderungen in der fetalen Gehirnentwicklung assoziiert zu sein. Unsere Studie zeigte erstmalig ein erweitertes CSP und einen erweiterten Seitenventrikel bei Feten von diabetischen Schwangeren. Diese Parameter könnten zukünftig als Screening-Marker für einen Gestationsdiabetes dienen.

\section{02.02 Evaluierung des unteren Gesichtswinkels (IFA), Maxilla-Mandibula-Nasen-Winkels (MMN) und der Prefrontal Space Ratio (PFSR) bei euploiden Feten im Vergleich zu Feten mit Trisomie 21 im ersten Trimenon}

\section{Autoren Tekesin I', Graupner O}

Institut 1 Pränatalmedizin Stuttgart; 2 Frauenklinik und Poliklinik, Klinikum rechts der Isar, Technische Universität München

DOI 10.1055/s-0038-1670403

Einführung Untersuchung der Reliabilität und Erstellung von Referenzkurven für den unteren Gesichtswinkel (,inferior facial angel“, IFA), Maxilla-Mandibula-Nasen-Winkel (MMN) und für die Prefrontal Space Ratio (PFSR) im Rahmen des Ersttrimesterscreenings. Vergleich von IFA, MMN und PFSR zwischen euploiden Feten und Feten mit Trisomie 21.

Material und Methoden In einer retrospektiven Studie wurden 200 euploide Feten und 74 Feten mit Trisomie 21 zwischen 11+0 und 13+6 SSW eingeschlossen. In gespeicherten zwei-dimensionalen mittsagitalen Profilbildern wurden IFA, MMN und PFSR bei euploiden Feten durch zwei Untersucher gemessen. Zur Untersuchung der Reliabilität der Messungen wurden die Limits of Agreement (LoA) nach Bland-Altman sowie Intraclass Correlation Coefficients (ICC) berechnet. Zum Vergleich der euploiden Feten und der Feten mit Trisomie 21 wurden Kovarianzanalysen mit der Scheitel-Steiß-Länge (SSL) als Kovariate durchgeführt.

Resultate Die ICCs zur Bestimmung der Intra-Observer-Reliabilität lagen für beide Untersucher für alle drei Maße über 0,75 (=excellent). Für die InterObserver-Reliabilität wurden folgende ICCs berechnet: IFA 0,74, MMN 0,77, PFSR 0,65. LoA der doppelten Messungen des erfahreneren Untersuchers wa- 
ren: IFA $\left[-5,4^{\circ} ; 7,1^{\circ}\right]$, MMN $\left[-2,4^{\circ} ; 1,8^{\circ}\right]$, PFSR $[-0,37 ; 0,40]$. Bei euploiden Feten waren der mittlere IFA $76,5^{\circ} \pm 6,3$, der mittlere MMN $15,4^{\circ} \pm 2,8$ und die mittlere PFSR $0,76 \pm 0,4$. Bei Feten mit Trisomie 21 waren die Mittelwerte $81,1^{\circ} \pm 8,5,16,8^{\circ} \pm 6,7$ und $0,60 \pm 0,8$. In der Kovarianzanalyse zeigte sich bei beiden Winkeln und der PFSR der Einfluss von SSL und Trisomie 21 als signifikant $(p<0,05)$.

Schlussfolgerung Die Bestimmung von IFA, MMN und PFSR sind im Rahmen des Ersttrimesterscreenings möglich. IFA und MMN waren bei Feten mit Trisomie 21 erhöht und die PFSR erniedrigt.

\section{02.03 Vesiko-amniale Shuntanlage im ersten Trimenon bei Megazystis- erste Erfahrungen mit einem neuen Shuntsystem}

\author{
Autoren Strizek $B^{1}$, Geipel $A^{1}$, Gembruch $U^{1}$, Berg $C^{1,2}$ \\ Institut 1 Abteilung für Geburtshilfe und Pränatalmedizin, \\ Universitätsfrauenklinik Bonn; 2 Pränatale Medizin, Universitätsfrauenklinik \\ Köln \\ DOI 10.1055/s-0038-1670404
}

Einführung Eine fetale Megazystis geht mit einer hohen pulmonalen Mortalität und Niereninsuffizienz bei den Überlebenden einher. Eine vesiko-amnialen Shuntanlage (VAS) wurde bisher meist im zweiten Trimenon durchgeführt. Seit 2014 gibt es in Deutschland ein neues intrauterines Shuntsystem zur VAS, das über eine $18 \mathrm{G}$ Punktionskanüle appliziert werden kann.

Ziel der Studie war die Machbarkeit einer VAS im ersten Trimenon, Komplikations- und Dislokationsraten sowie neonatales Outcome zu untersuchen.

Material und Methoden Retrospektive Studie aller Fälle einer VAS vor $14+0$ SSW von 2014-2017 mit dem Somatex IUS (Intrauterine Shunt, Somatex Medical Technologies, Germany). Allen Patientinnen mit einer fetalen Megazystis von $>15 \mathrm{~mm}$ wurde nach detaillierter Sonografie und interdisziplinärer Aufklärung eine VAS angeboten.

Resultate Es wurden 11 VAS im Durchschnitt mit $13+0$ SSW $(12+3-13+6)$ bei 8 männlichen und 3 weiblichen Feten durchgeführt. Die durchschnittliche Blasenlänge war 30,0 mm (21-50 mm). Alle Punktionen waren ohne vorherige Amnionauffüllung möglich. Der Karyotyp war in allen Fällen unauffällig. Es traten zwei IUFTs in der 13. und 18. SSW auf. 3 Paare beendeten die Schwangerschaft im zweiten bzw. dritten Trimenon bei schwerwiegenden Begleitfehlbildungen (Spina bifida, Hydrocephalus, Hirnblutung). In 3 Fällen kam es im Verlauf zu einer Shuntdislokation nach intraabdominal (mit 25, 29 und 30 SSW), in diesen Fällen erfolgte eine zweite abdomino-amniale Shuntanlage. Sechs $(54,5 \%)$ Kinder wurden lebend geboren mit durchschnittlich 33,5 (31 39) SSW. Es trat kein perinataler Todesfall auf. Bei zwei Feten bestand pränatal der Verdacht auf eine Kloakenfehlbildung, die sich nach der Geburt bestätigte. Die weitern 4 waren männliche Feten mit posteriorer Urethralklappe. Die Lungen- und Nierenfunktion bei Geburt war bei allen sechs Neonaten unauffällig.

Schlussfolgerung Lebendgeborene Kinder mit einer frühen Diagnose einer Megazystis profitieren möglicherweise von einer VAS im ersten Trimenon hinsichtlich einer Senkung der pulmonalen Mortalität und Verbesserung der renalen Funktion bei Geburt. Allerdings ist die Rate an komplexen Begleitfehlbildungen des uro-genitalen und gastrointestinalen Systems hoch.

\section{02.04 Analyse der No Call Results bei NIPTS (Panorama)}

Autoren Götte $\mathrm{M}^{1}$, Bianchi $\mathrm{M}^{1}$, lacoi $\mathrm{A}^{1}$, Enzensberger $\mathrm{C}^{1}$, Axt-Fliedner $\mathrm{R}^{1}$ Institut 1 Zentrum für Frauenheilkunde und Geburtshilfe, Universitätsklinikum Gießen \& Marburg, Abteilung für Pränatalmedizin, Gießen DOI 10.1055/s-0038-1670405

Einführung Das Screening auf Chromosomenstörungen durch NIPT's (Nicht invasive Pränataltests) ist heutzutage ein wichtiger Bestandteil des klinischen Alltags. Die Tests weisen eine hohe Testgüte auf. Bei einigen Patientinnen kann jedoch aus unterschiedlichen Gründen keine Auswertung erfolgen. Ziel dieser Arbeit ist eine Analyse aller No-Call-Ergebnisse aus unserer Klinik und die Detektion möglicher Gründe für diese „Testversager“.

Material \& Methoden Retrospektive Analyse aller NIPT's ( $n=387$ ) aus unserer Abteilung für Pränataldiagnostik am UKGM in Gießen. Der bei uns verwendete NIPT (Panoramatest, Natera) basiert auf der SNP-Methode (single-nucleotidepolymorphism), durch die fetale und maternale cfDNA unterschieden und gezielt die fetale cfDNA sequenziert und analysiert werden kann.

Resultate Im Zeitraum von 09/2916 bis 03/2018 gab es in unserer Abteilung insgesamt n= 387 NIPT's. Die No Call Rate aller NIPT's beträgt $n=20(5,17 \%)$, bei insgesamt 17 Kasuistiken, von denen 13 Fälle zwischen der 11+0-13+6 SSW waren. In 12/17 Fällen erfolgte die Re-Testung, bei der in 3 Fällen ein erneuter No Call resultierte, in sieben Fällen einen unauffälligen Befund und in einem Fall ein High Risk ergaben. In fünf Fällen wurde auf einen Re-NIPT (Panorama) verzichtet. Die Gründe für ein No Result im NIPT sind zum Beispiel eine zu niedrige fetal fraction, ein auffälliger Chromosomenbefund (z. B. Trisomie 21 , Triploidie) oder eine nicht adäquät durchführbare DNA-Sequenzierung.

Schlussfolgerung Die Testmöglichkeiten der verschiedenen NIPT's werden stetig weiterentwickelt. Auch die Testgüten sind, je nach NIPT und Labormethode, größtenteils sehr gut. Die Gründe für eine nicht auswertbare Probe sind mannigfaltig und zeigen Grenzen der Testmöglichkeiten auf.

\section{02.05 Untersuchung des Strain und der Dyssynchronie bei unauffälligen Feten mittels Speckle Tracking Echokardiografie - Vergleich von drei unterschiedlichen Ultraschallsonden}

Autoren Schmitz $\mathrm{R}^{1}$, Rolf $\mathrm{D}^{1}$, Schmidt $\mathrm{R}^{2}$, Möllers $\mathrm{M}^{1}$, Oelmeier de Murcia $\mathrm{K}^{1}$, Braun J $]^{1}$, Klockenbusch $W^{1}$, Hammer $K^{1}$

Institut 1 Klinik für Frauenheilkunde und Geburtshilfe, Bereich Geburtshilfe und Pränatalmedizin; 2 Institut für Biometrie und Klinische Forschung/1 - 2 Universitätsklinik Münster

DOI 10.1055/s-0038-1670406

Einführung In dieser Studie wird der Einfluss unterschiedlicher Ultraschallsonden auf die Messung des segmentalen myokardialen Strains des linken (LV-S) und rechten Ventrikels (RV-S) und der longitudinalen Dyssynchronie beider Ventrikel (2C-DYS) mittels Speckle Tracking Echokardiografie (STE) untersucht.

Material und Methoden Es wurden prospektiv bei 53 unauffälligen Feten STE Datensätze im Vierkammerblick mit drei unterschiedlichen Ultraschallsonden ( $5 \mathrm{MHz}$ Sector Array (S5 - 1), $8 \mathrm{MHz}$ Sector Array $(\mathrm{S} 8-3)$ und $5 \mathrm{MHz}$ xMatrix Array (X5 - 1); EPIQ 7 Ultraschall System, Philips Medical Systems, Andover, MA, USA) aufgenommen. Die Datensätze wurden anschließend offline mittels STE Analysesoftware (QLab 10.4, Philips Medical Systems) ausgewertet. Zusätzlich untersuchten wir den Einfluss der fetalen Herzfrequenzvariabilität. Resultate Die Messung des myokardialen Strains und der Dyssynchronie war mit den drei unterschiedlichen Sonden bei allen 53 eingeschlossenen Fällen möglich. Es zeigte sich kein Einfluss der verwendeten Sonden oder der Herzfrequenzvariabilität auf die Messergebnisse von LV-S, RV-S und 2C-DYS.

Schlussfolgerung Die verwendeten Ultraschallsonden zeigen keinen Einfluss auf die Ergebnisse der myokardialen Strain- und Dyssynchroniemessung. In weiteren Studien sollte der Nutzen einer STE Untersuchung für die pränatale Überwachung geprüft werden.

\section{02.06 Increased nuchal translucency and pregnancy outcome: a retrospective study}

Authors Tekesin $\mathrm{I}^{1}$ Institute 1 Pränatalmedizin Stuttgart DOI 10.1055/s-0038-1670407

Purpose To describe pregnancy outcome for fetuses with increased nuchal translucency (NT) in relation to the degree of increased NT. 
Methods In this retrospective study we analysed the data of pregnant women who were referred to our prenatal unit for first trimester screening for fetal aneuploidy. For fetuses with an NT $\geq 95$ th percentile, the frequencies of chromosomal abnormality, fetal malformation, intrauterine death (IUD) and termination of pregnancy (TOP) were determined in relation to the degree of NT thickness.

Results 7,352 pregnancies were analysed. An increased NT was detected in 397 (5.4\%) of the cases. The percentage of a favourable pregnancy outcome decreased from $77.8 \%$ in the lowest NT group (95th percentile-3.4 mm) to $5 \%$ in the highest NT group (NT $\geq 6.5 \mathrm{~mm}$ ). The frequency of chromosomal abnormalities rose markedly with increasing NT from $18.1 \%$ (lowest NT group) to $70 \%$ (highest NT group). In euploid fetuses, cardiac defects were the most common structural abnormalities, but additionally a wide range of structural and genetic defects was observed. Half of the cases with major abnormalities were observed in the highest NT group. In euploid fetuses in the lowest NT categories (NT up to $5.4 \mathrm{~mm}$ ), $80-95 \%$ of the pregnancies resulted in the delivery of a healthy child.

Conclusions Adverse pregnancy outcome rose with increasing NT. If karyotype was normal and the degree of NT thickness was low, the chance of a favourable outcome was still high.

\section{02.07 Einführung eines Studententutorbasierten geburtshilflichen und gynäkologischen Ultraschallbasiskurs in der Medizinlehre am UKS}

Autoren Hamza $A^{1}$, Radosa $]^{1}$, Takacs $Z^{1}$, Solomayer EF ${ }^{1}$, Ströder $R^{1}$, JuhaszBoess $\mathrm{I}^{1}$, Meyberg-Solomayer $\mathrm{B}^{1}$

Institut 1 Klinik für Frauenheilkunde, Geburtshilfe und Reproduktionsmedizin DOI 10.1055/s-0038-1670408

Hintergrund Die Einführung von Ultraschall in der Medizinlehre wird in den letzten Jahren stark gefördert. Auch die Anwendung von Studententutoren in der Lehre wird zunehmend unterstützt. Allerdings ist der Einsatz von Studententutoren im Bereich von Frauenheilkunde wenig untersucht.

Methoden Nach einer 3 wöchigen Ausbildung der Tutoren werden sie im Rahmen des Blockpraktikums für die praktische Ultraschallübung eingesetzt. Die Ziele und Inhalte der Ultraschallkurse wurden standardisiert. Die Studententutoren übernahmen daraufhin sowohl die theoretische als auch die praktische Einführung von Ultraschall in der Frauenheilkunde. Dies erfolgte unter der Aufsicht der zuständigen Oberärzte. Die Blockpraktikumstudenten erhielten ein Antetestat vor und ein Posttestat nach dem Kurs. Im Anschluss des Kurses erfolgte dessen Evaluation durch die Studenten mittels eines standardisierten Fragenbogens.

Resultate 111 Studenten haben an diesem Kurs teilgenommen. Der Kurs wurde von 3 Studententutoren unter ärztlicher Aufsicht geleitet. Sowohl im theoretischen als auch im praktsichen Teil des Ante- und Posttestates zeigten sich statistisch signifikante Notenverbesserungen (50 vs. $85 \%, p<0,05$ ). Im Fragebogen spiegelte sich eine große Zufriedenheit und subjektives Empfinden einer Verbesserung der Lehre und Praxis wider. Die Studenten haben die Tutoren sehr positiv bewertet.

Schlussfolgerung Die Studenten profitieren von studententutorenbasierte Ultraschallkurse in der Frauenheilkunde. Es besteht sowohl eine messbare Leistungsverbesserung als auch eine allgemeine Akzeptanz der Studententutoren seitens ihrer Kommilitonen.
Wi-Vo 03 Gynäkologie/Geburtshilfe II

Do. 15.11. 10:30 - 12:00 Shanghai 3

\section{03.01 Hygienische Aufbereitung von Ultraschallsonden mit einem Desinfektionstuchsystem in Anlehnung an den 4-Felder-Test und DIN EN ISO 17664}

Autoren Pahl $\mathrm{S}^{1}$, Steinmann $\mathrm{J}^{1}$, Henningsen $\mathrm{L}^{1}$, Becker $\mathrm{B}^{1}$, Bischoff $\mathrm{B}^{1}$, Brill FHH ${ }^{1}$, Klock JH $\mathrm{H}^{1}$, Gabriel $\mathrm{H}^{1}$

Institut 1 Dr. Brill \& Partner GmbH, Institut für Hygiene und Mikrobiologie, Hamburg

DOI 10.1055/s-0038-1670409

Einführung Ultraschallsonden zur trans-vaginalen Anwendung müssen nach dem Einsatz beim Patienten aufbereitet werden, um eine Infektionsübertragung zu verhindern. Üblicherweise erfolgt die Aufbereitung direkt nach der Anwendung und Entfernung des Kondoms manuell mit einem getränkten Desinfektionstuch. Unklar ist, ob es sich bei dieser Anwendung um eine Flächen- oder eine Instrumentendesinfektion handelt. Die Anwendung spricht eher für eine Flächendesinfektion, jedoch ist fraglich, ob die üblichen Prüfverfahren für Flächendesinfektionsmittel z.B. nach DIN EN 13697 auf Edelstahl, DGHM 2001, Methode 14 auf OP-Fliesen oder dem 4-Felder-Test auf PVC, eine valide Wirksamkeitsbewertung erlaubt. Daher sollte ein Prüfverfahren entwickelt werden, welches die praktische Anwendung für ein spezielles Produkt simuliert und einer praxisnahen Wirksamkeitsüberprüfung im Sinne einer Medizinprodukteaufbereitung nach der DIN EN ISO 17664 gerecht wird.

Material und Methoden Die geprüften Ultraschallsonden wurden an relevanten Stellen mit praxisrelevanten Prüforganismen z.B. Staphylococcus aureus, Escherichia coli, Candida albicans oder dem Polyomavirus SV 40 als Surrogat für humane Papillomaviren kontaminiert. Die Applikation des Desinfektionsmittels erfolgte mittels Tuch in einem standarisierten Verfahren. Nach der Desinfektion wurden die Prüforganismen von den zuvor kontaminierten Stellen zurückgewonnen, um die Desinfektionsleistung zu bestimmen. Außerdem wurde in Anlehnung an den 4-Felder-Test die Verbreitung der Prüforganismen an 3 weitere zuvor nicht kontaminierten Stellen der Ultraschallsonden überprüft.

Resultate Die Ergebnisse zeigen, dass das Prüfverfahren geeignet ist, um die Wirksamkeit zur manuellen Desinfektion von Ultraschallsonden zu überprüfen. Das eingesetzte Desinfektionsverfahren zeigte eine ausreichende Wirksamkeit. Eine Verbreitung der Prüforganismen war nicht nachweisbar.

Schlussfolgerung Es scheint sinnvoll in Zukunft spezielle Prüfverfahren einzusetzen, um die Wirksamkeit von innovativen Desinfektionsmitteln für spezielle Anwendungsgebiete im Sinne eine Phase 2, Stufe 2 oder Phase 3 Prüfung zu bewerten und somit den eine größere Anwendungssicherheit bei der Aufbereitung von Ultraschallsonden zu erreichen. Im Sinne einer sicheren Wirksamkeitsbewertung sind hierfür flexible Prüfansätze zu wählen, die den klinischen Einsatz der Produkte simulieren.

\section{03.02 Estimating risk of malignancy in adnexal masses with ultrasound: a retrospective diagnostic accuracy study}

Authors Reina $\mathrm{H}^{1}$, Vigo $\mathrm{F}^{1}$, Butenschoen $\mathrm{A}^{2}$, Buechel $]^{2}$, Schoetzau $A^{1}$, Heinzelmann-Schwarz $V^{1}$, Manegold-Brauer $G^{2}$

Institute 1 Gynecology; 2 Department for gynecologic and prenatal

ultrasound/1 - 2 University Women's Hospital Basel

DOI 10.1055/s-0038-1670410

Introduction The aim of this study is to evaluate the performance of ultrasound with pattern recognition by experts, Risk of Malignancy Index (RMI) and IOTA ADNEX in the differentiation between benign and malignant adnexal masses. 
Material and Methods This is a retrospective diagnostic accuracy study, based on data prospectively collected from patients with adnexal masses (Viewpoint data base), who underwent transvaginal and/or transabdominal examination by experienced examiners in our department between December 2016 and December 2017. The risk of malignancy, defined as invasive or borderline tumors, was determined by pattern recognition and the use of two prediction models: the ADNEX model and the Risk of Malignancy Index (RMI, cut off 200). Histological findings were the clinical reference standard. Results In the studied period, we recorded adnexal findings in 426 consecutive examinations. Of these, 122 had surgery and were included in the analysis. $60 \%$ of patients were premenopausal and $40 \%$ were postmenopausal. Seventy-seven percent of the masses were benign, $6 \%$ borderline-tumors, $16 \%$ invasive cancers. Expert pattern recognition and RMI had sensitivities of $87 \%$ and $80 \%$ at a specificity of $93 \%$ in differentiating malignant from benign tumors. The ADNEX model showed a sensitivity of $92 \%$ at a specificity of $86 \%$. Conclusions Although pattern recognition by experts seems to have the best discriminating power at our center, RMI and IOTA ADNEX help in preoperative planning and are a valuable tool for triage for referral and in teaching settings.

\section{03.03 Ultrasound-guided core needle biopsy (CNB) of pelvic masses in gynecology}

Authors Butenschön $A^{1}$, Reina $H^{1}$, Büchel $J^{1}$, Schönberger $H^{1}$, HeinzelmannSchwarz $V^{2}$, Manegold-Brauer $\mathrm{G}^{1}$

Institute 1 Division of Prenatal and Gynecologic Ultrasound; 2 Division of Gynecology and Gynecological Oncology/1-2 Department of Obstetrics and Gynecology, Basel University Hospital

DOI 10.1055/s-0038-1670411

Introduction Due to advances in treatment options for advanced ovarian or peritoneal malignancies, there is a need for histologic sampling prior to neoadjuvant chemotherapy for cytoreduction. A biopsy may also be indicated in patients with a history of gynecologic malignancy and a suspected disease recurrence. The aim of our study was to retrospectively evaluate the indications, feasibility, diagnostic accuracy and safety of CNB performed in our gynecologic cancer center.

Material and Methods We reviewed the medical records of eight patients who underwent transvaginal or transabdominal US-guided core biopsy between May 2017 and April 2018. All biopsies were performed with an automatic biopsy gun (BARD ${ }^{\circledR}$ ) after a detailed gynecologic ultrasound and informed consent. For the transvaginal approach, an attached needle guide and 18 Gauge $/ 25 \mathrm{~cm}$ needles was used. Transabdominal biopsies were performed under local anesthesia with $14-16$ Gauge/ $15-20 \mathrm{~cm}$ needles. We evaluated the indications, diagnostic accuracy and complication rate of the procedures.

Results Six transvaginal and two transabdominal CNBs were performed during the study period. Indications for CNB were patients with advanced ovarian, primary inoperable malignancies $(n=3)$, inoperable patients due to comorbidities $(n=2)$ and patients with suspicion of metastases or recurrence of gynecologic malignancies $(n=3)$. Representative samples were obtained in all cases. All procedures were tolerated with minimal discomfort on an outpatient basis. Of the 8 lesions, 7 were confirmed to be either benign $(n=2)$ or malignant $(n=5)$. One lesion was not histopathologically classified after biopsy. A CT scan guided biopsy showed adiponecrosis. No complications occurred during or after CNB procedures.

Conclusion Transvaginal and transabdominal ultrasound guided biopsy seems to be a feasible and safe procedure for histopathologic diagnosis of pelvic masses.

\section{03.04 Beurteilung des intrauterinen Verlaufs, assoziierter Anomalien und des postnatalen Outcomes von Feten mit Pulmonalatresie mit Ventrikelseptumdefekt (PAVSD)}

Autoren Gottschalk I ${ }^{1}$

Institut 1 Abteilung für Pränatale Medizin und Gynäkologische Sonografie, Universitätsfrauenklinik Köln

DOI 10.1055/s-0038-1670412

Einführung Beurteilung des intrauterinen Verlaufs, assoziierter Anomalien und des postnatalen Outcomes von Feten mit Pulmonalatresie mit Ventrikelseptumdefekt (PAVSD).

Methoden Retrospektive Auswertung aller über einen Zeitraum von 10 Jahren in den Universitätsfrauenkliniken Köln und Bonn sowie bei pränatal.de diagnostizierten Feten mit PAVSD und einem postnatalen Follow-up von mindestens 6,5 Jahren.

Resultate 50 PAVSD wurden pränatal diagnostiziert. 44\% der Feten hatten isolierte PAVSD, $4 \%$ hatten assoziierte kardiale und 10\% extra-kardiale Anomalien, $38 \%$ hatten chromosomale Anomalien und 4 nicht-chromosomale Syndrome.

Von den 32 Lebendgeborenen hatten 57\% der Kinder einen reversen Fluss über einen offenen Ductus arteriosus, $25 \%$ hatten major aortopulmonale Kollateralarterien (MAPCAs) mit Ductusagenesie und 18\% hatten einen double supply. 17 (34\%) Schwangerschaften wurden abgebrochen, 1 Fet (2\%) syndromaler Fet verstarb intrauterin, 1 Kind mit Trisomie $18(2 \%)$ in der Neonatalzeit, 6 Kinder (12\%) im Säuglingsalter während oder nach kardio-chirurgischer Korrektur mit Ausnahme eines syndromalen Fet nach rein palliativer postpataler Versorgung. 25 von 30 (83,3\%) Lebendgeborenen mit intentionto-treat lebten beim letzten Follow-Up. Das durchschnittliche Follow-Up unter den Überlebenden betrug 10,0 Jahre (range 6,5 - 15,1). 56\% der Kinder erhielten eine zweizeitige Korrektur, $44 \%$ wurden einzeitig operiert. Nach Ausschluss der Kinder mit chromosomalen oder syndromalen Anomalien waren 89\% der Kinder nach kardiochirurgischer Korrektur gesund ohne Einschränkungen, $11 \%$ leicht beeinträchtigt. Die Inzidenz von MAPCAs unterschied sich nicht signifikant zwischen Verstorbenen und Überlebenden $(p=0,360)$, zwischen einzeitig und zweizeitig operierten $(p=0,656)$ und zwischen gesunden und beeinträchtigten Kindern $(p=0,319)$. Nach zweizeitiger Korrektur benötigten $93 \%$ der Kinder Re-Interventionen aufgrund von rekurrierenden ShuntStenosen, nach einzeitiger Korrektur nur 36\% der Kinder.

Schlussfolgerung Die Langzeit-Prognose der PAVSD ohne assoziierte chromosomale oder syndromale Anomalien ist gut, die überwiegende Mehrzahl der Kinder überlebt ohne Einschränkungen. Die post-operative Gesundheit ist unabhängig von der Art der OP gut. MAPCAs beeinflussen weder die Prognose noch den postoperativen Gesundheitszustand. Lediglich die Inzidenz und Anzahl wiederholter Interventionen durch rekurrierende Stenosen war nach zweizeitiger operativer Korrektur signifikant häufiger nötig als nach einzeitiger Korrektur.

\section{03.05 Prenatal diagnosis and postnatal outcome of fetuses with double outlet right ventricle (DORV) in a single center}

\section{Authors Gottschalk I}

Institute 1 Abteilung für Pränatalmedizin und Gynäkologische Sonografie, Universitätsfrauenklinik Köln

DOI 10.1055/s-0038-1670413

Purpose To assess the spectrum of associated anomalies, the intrauterine course, the postnatal outcome and management of fetuses with DORV.

Methods All cases of DORV diagnosed prenatally over a period of 8 years were retrospectively collected in a single tertiary referral center in Germany. All additional prenatal findings were assessed and correlated with the outcome. The morphological variants of DORV and the accuracy of prenatal diagnosis was assessed. 
Results 46 cases of DORV were diagnosed prenatally. Mean gestational age at first diagnosis was $21+4$ weeks (range, $13-37$ ). 1 case had TGA with VSD postnatally and was excluded from the outcome. All 45 fetuses with DORV $(100 \%)$ had major additional cardiac anomalies, 30 (66.7\%) had extra-cardiac anomalies and 13 (28.9\%) had chromosomal or syndromal anomalies. Due to their complex additional anomalies, 5 (11.1\%) of our 45 fetuses were highly suspicious for non-chromosomal syndromes, although molecular diagnosis could not be provided. There were 17 terminations of pregnancy (37.8\%), 2 (4.4\%) intrauterine and 7 (15.6\%) postnatal deaths. 19/22 (86.4\%) live born children with an intention to treat were alive at last follow up. Mean follow up among survivors was 32 months (range, 2 - 72). 20/22 children underwent postnatal sugery, 8 (40.0\%) achieved biventricular repair, 12 (60.0\%) univentricular palliation and 2 recently born children are waiting for surgery. After surgery, 14/17 (82.4\%) children were healthy without any limitations. Correct prenatal diagnosis of DORV was made in $26 / 27$ (96.3\%). If the relation of great arteries, position of VSD and additional cardiac and extra-cardiac anomalies are taken into account, the prenatal diagnosis was correct in 24 / $27(88.9 \%)$ cases.

Conclusion DORV is a rare and often complex cardiac anomaly that can be diagnosed prenatally with high precision. DORV is frequently associated with major additional anomalies, leading to a high intrauterine and postnatal loss rate due to intrauterine terminations or declined postnatal therapy. Without these anomalies, prognosis is good, although $60 \%$ of children will achieve single ventricle palliation.

\section{03.06 Messung der fetalen Arteria cerebri media - Bedeutung der Doppler Presets}

Autoren Schmitz $\mathrm{R}^{1}$, Wibbeke $\mathrm{D}^{1}$, Hammer $\mathrm{K}^{1}$, Möllers $\mathrm{M}^{1}$, Köster $\mathrm{HA}^{1}$, Falkenberg $\mathrm{MK}^{1}$, Borowski $\mathrm{M}^{2}$, Klockenbusch $\mathrm{K}^{1}$, Oelmeier de Murcia $\mathrm{K}^{1}$ Institut 1 Klinik für Frauenheilkunde und Geburtshilfe, Bereich Geburtshilfe und Pränatalmedizin; 2 Institut für Biometrie und Klinische Forschung/1-2 Universitätsklinik Münster

DOI 10.1055/s-0038-1670414

Einführung Die dopplersonografische Messung der Arteria cerebri media (ACM) spielt eine zentrale Rolle in der Überwachung von Hochrisikoschwangerschaften. Ziel der Studie war es, die Bedeutung von Doppler Presets für die Messung der maximalen Blutflussgeschwindigkeit (Vmax) und des Pulsatilitätsindex (PI) zu untersuchen.

Material und Methoden PI und Vmax der ACM wurden prospektiv an 350 gesunden Feten zwischen der 19. und 42. Schwangerschaftswoche dopplersonografisch untersucht. Die erste Messung der ACM (MCA.S) wurde mit der konventionellen Einstellung durchgeführt. Die zweite Messung (MCA. O) wurde mit einer optimierten Einstellung und einer maximal erreichbaren Frame Rate gemessen. Für die MCA.O Messung wurde der Scanbereich des BMode Bildes und das Farbdopplerfenster so schmal wie möglich angepasst. Zusätzlich wurde die ACM im High Definition Zoom dargestellt. Die daraus resultierenden Messungen wurden mit den bisher geltenden Referenzwerten verglichen.

Resultate Vmax, PI als auch die Framerate der MCA.O Messeinstellung waren höher als die Messwerte der MCA.S Messeinstellung ( $P<0,001$ für alle Parameter). Für beide Preseteinstellungen zeigten sich die Messwerte erhöht im Vergleich zu den geltenden Referenzwerten.

Schlussfolgerung Die Messung der ACM mit optimierter Doppler Preseteinstellung zeigt höhere Messwerte der Vmax und des PI verglichen mit der herkömmlichen Messmethodik. Zudem unterscheiden sich die Mediankurven von den bisherigen Referenzkurven. Die die Standards für die Durchführung der ACM-Messung und die Referenzwerte sollten aktualisiert werden.

\section{03.07 Kongenitale porto-systemische Shunts - postnatale Persistenz und Verlauf nach pränataler Diagnose}

Autoren Strizek $B^{1}$, Gottschalk $I^{2}$, Geipel $A^{1}$, Müller $A^{3}$, Gembruch $U^{1}$, Berg $C^{1,2}$

Institut 1 Abteilung für Geburtshilfe und Pränatalmedizin,

Universitätsfrauenklinik Bonn; 2 Pränatale Medizin, Universitätsfrauenklinik

Köln; 3 Neonatologie, Universitäts-Kinderklinik Bonn

DOI 10.1055/s-0038-1670415

Einführung Die pränatale Diagnose kongenitaler Fehlbildungen des Portalsystems ist selten. Aufgrund der großen Variabilität des hepatischen Venensystems und der ausgeprägten postnatalen Strömungsveränderungen ist der Verlauf v.a. im Hinblick auf die postnatale Ausbildung des Portalsystems oft schwierig vorherzusagen. Kongenitale portosystemische Shunts (PSS) können zu schwerwiegenden Komplikationen postnatal (Hyperammonämie, Encephalopathie und Rechtsherzbelastung) führen.

Material und Methoden Retrospektive Analyse aller pränatal diagnostizierten Fälle in zwei Perinatalzentren von 2009 - 2016 im Hinblick auf assoziierte Fehlbildungen, den intrauterinen Verlauf, die postnatale PSS-Persistenzrate sowie Therapie. Ein PSS wurde als die Persistenz einer Shuntverbindung des Portalsystems zum Zeitpunkt der Entlassung aus der Geburtsklinik definiert.

Resultate Von 69 Fällen mit der pränatalen Diagnose eines PSS trat dieser bei 23 Feten (33\%) isoliert auf, bei allen anderen lagen chromosomale, syndomale oder andere isolierte Fehlbildungen vor. In 8 Fällen wurde eine VACTERL-Assoziation beschrieben. In 3 Fällen fand sich ein normaler Ductus venosus, bei allen anderen lag auch eine Ductus venosus-Agenesie (DVA) vor. 36 Kinder überlebten, es gab 4 IUFT, 11 postnatale Todesfälle und 18 Schwangerschaftsabbrüche aufgrund assoziierter Fehlbildungen. Ein Kind mit einer totalen portalen Agenesie verstarb im Rahmen einer Herz-Operation, alle weiteren präund postnatalen Todesfälle traten bei Kindern mit assoziierten Fehlbildungen oder Erkrankungen auf. Bei den isolierten Fällen verschloss sich in $61 \%$ die Shuntverbindung unmittelbar nach der Geburt. Von insg. 9 PSS verschlossen sich 2 spontan und 2 wurden interventionell in den ersten 2 Lebensjahren verschlossen. 4 Kinder weisen einen PSS ohne hämodynamische Auswirkungen auf und bei einem Kind besteht eine Abernathy 2-Malformation.

Schlussfolgerung Alle Neugeborenen mit pränatalem Verdacht auf einen PSS sollten regelmäßige sonografische Kontrollen erhalten. Der isolierte portosystemische Shunt hat unabhängig vom Vorliegen einer DV-Agenesie eine hohe spontane Verschlussrate. Interventionen sind nur in einer Minderheit der Fälle notwendig. Eine totale portale Agenesie ist selbst bei DVA mit Leberbypass sehr selten.

Wi-Vo 04 Technik/Qualität/Sicherheit

Do. 15.11. 10:30 - 12:00 Boston 3

\section{04.01 KoMBUS - MR-compatible ultrasound for improved biopsy needle guidance}

Authors Fournelle $\mathrm{M}^{1}$, Hewener $\mathrm{H}^{1}$, Speicher $\mathrm{D}^{1}$, Günther $\mathrm{M}^{2}$, Buecker $\mathrm{A}^{3}$, Tretbar $S^{1}$

Institute 1 Fraunhofer IBMT, Sankt Ingbert; 2 Fraunhofer MEVIS, Bremen; 3 Universitätsklinikum des Saarlandes, Homburg

DOI 10.1055/s-0038-1670416

Biopsies have become an essential tool for minimally invasive diagnostics and are part of the daily routine of interventional radiologists. Depending on the target organ that has to be sampled, different imaging modalities such as CT, ultrasound or MRI can be used. In particular, when it comes to detection of small tumors having a low contrast in ultrasound or CT, MRI is the method of choice. The risk for being off-target leading to a potentially false-negative diagnosis is reduced due to the better contrast on MR images. However, 
MRI still has drawbacks when compared to ultrasound with respect to cost issues, patient comfort and time-resolution.

The goal of KoMBUS therefore is to develop MR-compatible ultrasound hardware allowing to replace MRI by ultrasound to the greatest possible extent in the context of biopsy guidance. In this concept, a diagnostic MRI scan will be performed prior to the biopsy with a simultaneous 3D US scan of the ROI. The latter will be used to autonomously follow the tissue motion and deformation (e.g. as a result of breathing) based on the tracking of defined tissue landmarks. The fusion of the initial MR data and the real-time tracking information derived from US data allows to generate synthetic MR data sets, which reflect the current anatomical situation. If the ultrasound probe is connected to a mechanical needle guiding system, our therapy planning software module will calculate the accurate needle path for a defined target and a corresponding breathing phase. The concept will allow precise biopsies while only needing one initial MRI scan, which reduces the patient discomfort associated with repetitive MR-scans during the biopsies and saves MR time.

In view of the clinical validation of this concept, an MR compatible ultrasound platform consisting of a 256 channel system, different MR-compatible probes and an MR-compatible touchscreen has been developed. For tracking of the organ deformation, an $11 \times 11$ elements matrix array probe for acquisition of volume data that will be fed in our motion tracking algorithms, has been developed. The development and characterization of our hard- and software will be presented as well as first measurements on phantoms. Furthermore, a clinical validation of the concept is in preparation and the first tests for compliance with the medical device directive have been performed.

\section{04.02 Sono-Teleconsulting}

Autoren Ortmann $S^{1}$, Haddadi-Esfahani $A^{1}$, Frauendorf $V^{2}$, Nürnberg $D^{2}$ Institut 1 IHP, Frankfurt (Oder); 2 Brandenburg Medical School Theodor Fontane, Neuruppin DOI 10.1055/s-0038-1670417

Einführung Im Zuge des technologischen Fortschritts sind mittlerweile auch komplexere Ultraschallgeräte als portable Varianten verfügbar, womit sich Point-of-Care-Ultraschall (POCUS) Anwendungen in der Akut- und Flächenversorgung umsetzen lassen. Dabei bremst aktuell nicht ein technischer sondern personeller Engpass an hochqualifiziertem Personal diese Entwicklungen aus. Das hier vorgestellte Konzept des Sono-Teleconsulting erlaubt die Verwendung eines mobilen POCUS Gerätes in der Flächenversorgung mit zusätzlicher Live-Konferenzschaltung zu einem Ultraschall-Experten, der in seiner Klinik/ Ambulanz verbleibt.

Material und Methoden Ziel Sono-Teleconsulting ist es, nicht nur die reinen Ultraschallbilder an einen Experten zu übermitteln, sondern simultan auch einen visuellen Eindruck über den Patienten, die Handhabung des Ultraschallgerätes, den durchgeführten Schnitt sowie eine Sprachverbindung in einer Live-Konferenz zwischen dem vor-Ort Behandler und dem zugeschalteten Experte zu ermöglichen. Dazu werden die Bilder aus dem Ultrasound Gerät mit parallelen Bilddaten einer weiteren Kamera synchronisiert, zusammengeführt und während der Ultraschalluntersuchung live übermittelt.

Resultate Eine erste Anwendung wurde mit dem Ultraschallgerät des amerikanischen Herstellers Interson erfolgreich umgesetzt. Diese Beispielanwendung erlaubt dem Remote Experten zusätzlich sogar die Steuerung/Justierung des Ultraschallgerätes beim Behandler vor Ort. Derzeit wird das Konzept durch die Umsetzung einer Streaming-Schnittstelle erweitert, so dass auch Bilddaten verschiedener anderer Hersteller/Geräte drahtlos in das gleiche Nutzerinterface integriert werden können. Für die Remoteanbindung werden derzeit mind. 2 Mbit Übertragungsgeschwindigkeit benötigt um eine minimale Bildwiederholrate von 20 Frames pro Sekunde zu erreichen. Die entspricht etwa $1 / 3$ der Bitrate einer $3 G$ Anbindung mittels HSDPA Standard.

Schlussfolgerung Unser Konzept zeigt, dass bereits bei 30\% Auslastung einer 3G Mobilfunkanbindung eine Remote-Ultraschallkonferenz zwischen dem ambulanten Behandler vor Ort und dem entfernt supervisierenden Experten technisch umsetzbar ist.

\section{04.03 Kurz- und langfristige Lerneffekte eines universitären modularen Ultraschallausbildungskonzeptes}

Autoren Holzmann $C^{1}$, Vennemann $S^{2}$, Marten-Mittag $B^{3}$, Vo Cong $\mathrm{M}^{1}$, Berberat $\mathrm{P}^{2}$, Stock $\mathrm{K}^{1}$

Institut 1 Nephrologischer Ultraschall, Abteilung für Nephrologie; 2 TUM Medical Education Centre, Fakultät für Medizin der Technischen Universität München; 3 Klinik und Poliklinik für Psychosomatische Medizin und Psychotherapie/1,3 Klinikum rechts der Isar der Technischen Universität München

\section{DOI 10.1055/s-0038-1670418}

Einführung Zurzeit gibt es im Medizinstudium Deutschland keine verpflichtende, einheitliche Ultraschallausbildung. Ultraschall ist jedoch eine der am häufigsten genutzten Bildgebungsmodalitäten in Deutschland. Vor dem Hintergrund der hohen Untersucherabhängigkeit erscheint ein gutes Ultraschalltraining, dessen Grundstein im Studium gelegt wird, überaus wichtig. Das Lehrkonzept an der XXX besteht aus einem verpflichtenden theoretischen Seminar und einem freiwilligen praktischen Tutorium mit Peer-Teaching-Ansatz. Zur Beurteilung des Konzepts wurden die Auswirkungen auf Wissen, Fähigkeiten und die Haltung der Studenten zur Methode Ultraschall untersucht.

Material und Methoden Die Evaluierung erfolgte zu den Zeitpunkten T0 (direkt vor der Intervention), T1 (direkt nach der Intervention) und T2 (4-6 Monate nach der Intervention). Es wurden Fragebögen, Multiple-Choice-Fragen und praktische Leistungstests wurden verwendet.

Resultate Das praktische Tutorial wurde von den Studenten mit einer mittleren Note von 1,1 (SD 0,3) und das theoretische Seminar mit 1,5 (SD 0,7) im Mittel bewertet. In den praktischen Fertigkeiten zeigte sich bei den prozeduralen Fähigkeiten (Vorbereitung und Interaktion, Orientierung im Bild, Umgang mit dem Ultraschallgerät) ein langfristiger Lerneffekt mit im Mittel 6,2 aus 14 Punkten (T0; SD 1,5), 11,7 (T1, SD 1,2, p<0,001) und 10,5 (T2, SD 1,4, $\mathrm{p}<0,001)$. Die sehr vom dargestellten Organ abhängige Bildqualität zeigte nur eine kurzfristige Verbesserung: im Mittel 6,8 aus 15 (SD 2,3, T0), 9,3 (SD 1,9, T1, p<0,001), 7,2 (SD 1,9, T2). Der Wissenstest mit MultipleChoice-Fragen nach dem theoretischen Seminar ergab einen langfristigen Lerneffekt: im Mittel 4,8 aus 10 ( $S D \pm 2, T 0$ ), 8,4 ( $S D \pm 1,2, T 1, p<0,001$ ), $7,4(S D \pm 1,5, T 2, p<0,001)$. Die Mehrheit der Studenten bewertete die Methode Ultraschall als dem CT oder MRT nicht oder kaum unterlegen. Praktisches Tutorium: T0 57\%, T1 67\%, T2 74\%, theoretisches Seminar: T0 70\%, T1 $72 \%$, T2 $77 \%$.

Schlussfolgerung Das Lehrkonzept der XXX zeigt Auswirkungen auf Wissen, praktische Fähigkeiten und Haltung. Der Peer-Teaching-Ansatz mit sehr gut ausgebildeten studentischen Instruktoren und sehr kleinen Lerngruppen ist in Zeiten knapper Ressourcen ein sinnvolles Instrument, jedoch sollten die Trainingsmöglichkeiten erweitert werden, um die Bildqualität langfristig zu verbessern. Mit einem theoretischen Seminar kann eine solide Wissensbasis geschaffen und die Haltung zur Methode Ultraschall verbessern.

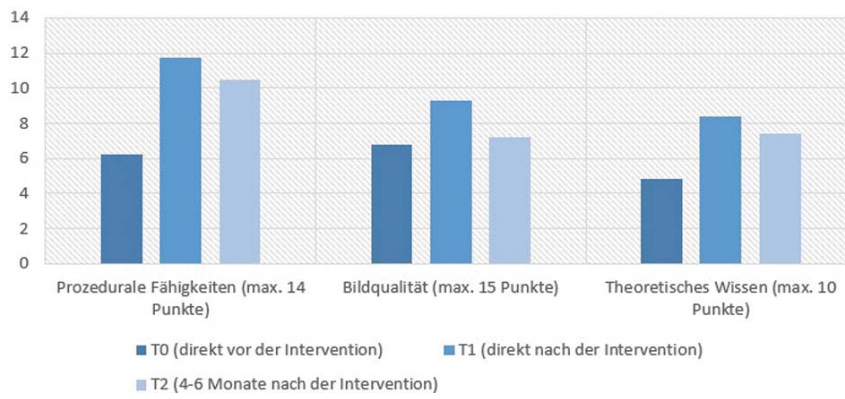

Abb. 1 Praktische und theoretische Lerneffekte des modularen Ultraschalltrainingskonzeptes 


\section{04.04 Mehrwert von EUS gesteuerten Fein-Nadel-Biopsien (FNB) gegenüber FN-Aspiration (FNA) von unklaren Läsionen im Gastrointestinal (GI)-Trakt}

Autoren Sivanathan $\mathrm{V}^{1}$, Schad $\mathrm{A}^{2}$, Thomaidis $\mathrm{T}^{1}$, Rahman $\mathrm{KF}^{1}$, Thieringer $\mathrm{F}^{1}$, Nguyen-Tat $\mathrm{M}^{1}$, Marquardt $\mathrm{JU}^{1}$, Roth $\mathrm{W}^{2}$, Galle $\mathrm{PR}^{1}$, Neumann $\mathrm{H}^{1}$, Möhler $\mathrm{M}^{1}$ Institut 1 I. Medizinischen Klinik und Poliklinik; 2 Institut für Pathologie der Johannes Gutenberg-Universität

DOI 10.1055/s-0038-1670419

Einleitung Im GI-Trakt sind für die gute Diagnostik und Therapieplanung von unklaren Läsionen/Tumoren die Charakterisierung mit Immunhistochemie (IC) und Molekularpathologie (MP) sinnvoll. Die Ergebnisqualität der EUS gesteuerten Biopsie (FNB) versus (vs.) der FN-Aspiration (FNA) für die IC und MPAufarbeitung war bisher unklar.

Methode Von 06/2017-04/2018 sammelten wir prospektiv, die an unserem Zentrum durchgeführten EUS-FNB (Acquire ${ }^{\mathrm{TM}}$ FNB Device, $22 \mathrm{G}$ ) und -FNA (EchoTip ${ }^{\circledR}$ Ultra, 22 \& $19 \mathrm{G}$ ) zur Abklärung von unklaren oder auffälligen Läsionen im oberen Gl-Trakt und werteten sie mit der Pathologie retrospektiv aus. Die EUS-Proben wurden sofort vor Ort in flüssigem Formalin fixiert.

Ergebnisse 51 Patienten (Pat.) (23 Frauen, 28 Männer; median 63 Jahre) wurden eingeschlossen. Die mit FNA und -FNB erhobenen Befunde waren: Unspezifisch ( $n=11 / 22 \%$ - untersuchte Organe (u.O.): 2xLymphknoten (LK), 3xMagen/Ösophagus, 6xPankreas), Pankreatitis (7/14\%), Pankreaskarzinom (7/ $14 \%)$, unauffälliges Lymphgewebe (6/12\%), dysplastische Zellen ohne sicheren Karzinomnachweis (3/6\% - u.O.: 1xGallengang, 1xLK, 1xPankreas), normales Pankreasgewebe (3/6\%), NET des Pankreas (2/4\%) und Magen-GIST (2/4\%). Einen wegweisenden Befund ergab der histopathologische Befund mit 32 FNB in 18 (56\%) und mit 25 FNA in 14 (56\%) Pat.. Die zielführende Differenzierung (Diff.) gelang bei 15 (29\%) erst durch zusätzliche IC. $67 \%$ dieser Proben wurden mit FNB gewonnen. Nur so gelang die Unterscheidung von Pankreas- vs. Bronchial-Karzinom (Сa.), Pankreas-Ca. ohne Mikrosatelitten-Instabilität (MSI) zum Ausschluss eines HNPCC, Pankreas- vs. Kolorektales-Ca., Urothel- vs. Gallengangs-Ca., Klärung eines NET (G1), eine Metastase (LK-Met.) eines GI-Ca., LK-Met. eines Kleinzelligen-Bronchial-Ca., Nebennieren-Met. von Ösophagus-Ca., Pankreatitis ohne IgG4-AIP, Pankreaszyste: Met. eines Ovarial-Ca., Pankreatitis nach Ausschluss NET und GIST. Nur bei 3 FNB, aber nicht FNA-Proben, konnte durch Genpanel-Analysen die Diff. eines GIST und bei einem Pankreas-Ca. die für die HNPCC typischen MSI-Loci bestimmt werden. Einzige Komplikation nach EUS-Punktionen waren 2 (4\%) leichte und konservativ behandelte Blutungen.

Schlussfolgerung Die EUS-FNB verbesserte in 2/3 der Fälle im Vergleich zur FNA (1/3) die Diagnostik durch zusätzliche IC und MP. Diese bessere Charakterisierung für Läsionen/Tumoren mit unbekanntem Primarius ermöglichte eine individuell angepasste Therapieplanung.

\section{04.05 Three different ways of training ultrasound student-tutors yield significant and comparable gains in tutee's scanning-skills - Weiterentwicklung curricularer Ultraschallkurs Innere Medizin um Notfallsonografie}

Authors Celebi $N^{1}$, Griewatz $]^{2}$, Riessen $\mathrm{R}^{3}$, Malek $\mathrm{N}^{4}$, Schmidt $\mathrm{T}^{4}$, Fröhlich $\mathrm{E}^{4}$ Institute 1 PHV dialysis center Waiblingen; 2 Competence Centre for University Teaching in Medicine, Baden-Wuerttemberg; 3 Department of Internal Medicine VIII (Intensive Care Unit); 4 Department of Internal Medicine I (Gastroenterology, Hepatology, Infectious Diseases)/3-4 University Hospital Tübingen

DOI 10.1055/s-0038-1670420

Introduction Many medical universities rely these days on trained student tutors to enable faculty-wide undergraduate ultrasound training. However, there is neither consensus on an optimal method nor any developed and agreed standard in the training of these student tutors. Usually internships and courses are employed which both have a specific set of advantages and disadvantages. We conducted a prospective quasi-randomized study to assess the effects of three types of tutor training on the resulting improvement in scanning skills of their tutees.

Methods Three batches of student tutors were trained by a course only ( $C$ group), by an internship only (I-group) or by a course and an internship (Clgroup). The respective gains in ultrasound scanning skills of the tutees were measured prospectively. A total of 75 (60.5\%) of the 124 th year medical students who attended the mandatory ultrasound course completed both pre- and post-exams on a voluntary basis. Within a limit of eight minutes and three images, they were asked to depict and label a maximum of 14 anatomical structures. Two blinded raters independently awarded two points for each label with an identifiable structure and one point for each label with a possibly identifiable structure.

Results In all three groups, the tutees improved significantly by more than doubling their pre-score results and comparably (Gains: C-group $9.19 \pm 5.73$ points, $\mathrm{p}<0.0001$, I-group $9.77 \pm 4.81$ points, $\mathrm{p}<0.0001$, Cl-group 8.97 \pm 5.49 points, $p<0.0001$ ).

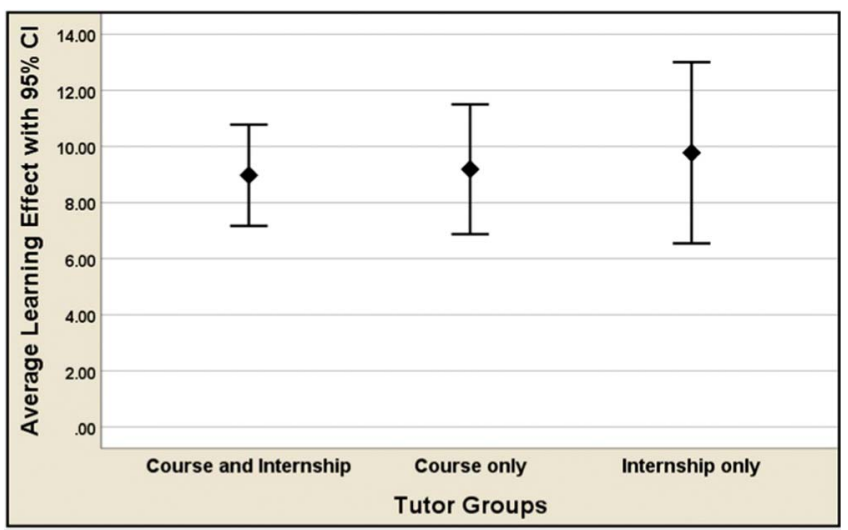

Dig. 1 Mean Tutees' Improvements in Scanning Skills OSCE Scores (with $95 \% \mathrm{Cl}$ ) by Tutor Training Groups ( $p<0.001$ for each group)

Conclusion Student tutors, who were trained with a course or an internship or a course and an internship could teach scanning skills to 5th year medical students very effectively and with similar success.

Wi-Vo 05 Kopf/Hals/Thorax

Do. 15.11. 14:00 - 15:30 Shanghai 1

\section{05.01 Elastografie bei der Differenzierung von Lymphknotenerkrankungen im Kopf-Hals-Bereich}

Autoren Mansour $\mathrm{N}^{1}$, Hofauer $\mathrm{B}^{1}$, Knopf $\mathrm{A}^{1}$

Institut 1 Klinikum rechts der Isar der Technischen Universität München,

Klinik und Poliklinik für Hals-, Nasen- und Ohrenheilkunde

DOI 10.1055/s-0038-1670421

Einführung Lymphknotenerkrankungen im Kopf-Hals-Bereich bedürfen unterschiedlicher Therapien, die sich grundsätzlich voneinander unterscheiden. Daher ist die präoperative Antizipation der zu erwartenden Entität von klinischer Bedeutung. Die hochauflösende Sonografie ist im diagnostischen Algorithmus Methode der Wahl. Wir evaluieren den Stellenwert der Elastografie in der Differenzierung von Lymphknotenerkrankungen.

Material und Methoden Es wurden 29 Patienten mit klinisch und sonografisch auffälligen Lymphknoten eingeschlossen (Acuson S2000, Siemens Medical Solutions USA, Inc., CA, USA). Es erfolgte eine retrospektive Analyse der BBild-Kriterien und FKDS. Zusätzlich wurden elastografische Verfahren angewendet und ihr Nutzen zur weiteren Differenzierung beurteilt. 
Resultate Es wurden sechs gesunde Lymphknoten (LK), dreizehn Lymphome, sieben Plattenepithelkarzinommetastasen und drei Lymphadenitiden eingeschlossen. Die gesunden LK waren in der Kompressionselastografie (SE) tendenziell eher weich, während die Plattenepithelkarzinommetastasen eher hart, die entzündlich veränderten LK und Lymphome weich bis indifferent waren. Doch die Unterschiede erreichten statisch nicht das Signifikanzniveau $(p=0,063)$. In der Scherwellenelastografie (SWE) gab es 13 nicht-messbare LK, davon waren 6 Plattenepithelkarzinommetastasen, 4 Lymphome und alle Lymphadenitiden. Es zeigte sich kein signifikanter Unterschied zwischen den Scherwellengeschwindigkeiten der gesunden LK und den Lymphomen $(p=0,083)$.

Schlussfolgerung Die elastografischen Ergebnisse zeigen zu große Überlappungen zwischen den verschiedenen Lymphknotenerkrankungen, so dass die Elastografie noch kein Routineverfahren in der Differenzierung von Lymphknotenerkrankungen darstellt.

\section{05.02 Sonografische Evaluation anatomischer Landmarken bei Patienten mit obstruktiver Schlafapnoe}

Autoren Hofauer $B^{1}$, Karl J $J^{1}$, Mansour $N^{1}$, Heiser $C^{1}$, Knopf $A^{1}$

Institut 1 Klinik und Poliklinik für Hals-Nasen-Ohrenheilkunde, Klinikum rechts der Isar der Technischen Universität München

DOI 10.1055/s-0038-1670422

Einleitung Die obstruktive Schlafapnoe (OSA) stellt bei einer weltweit steigenden Prävalenz die häufigste schlafbezogene Atmungsstörung dar. Neben der Tagesschläfrigkeit als Hauptsymptom geht die OSA mit einem erhöhten Risiko für kardiovaskuläre Erkrankungen einher. Die nächtliche CPAP-Therapie (continuous positive airway pressure) ist die Standard-Therapie dieser Patienten, jedoch existiert eine Vielzahl sowohl konservativer als auch chirurgischer Therapiealternativen. Ziel dieser Studie ist die Evaluation anatomischer Landmarken mittels Sonografie bei Patienten mit OSA und die Korrelation mit dem Schweregrad der Erkrankung.

Material und Methoden Patienten mit einer leicht-, mittel- und schwergradigen OSA wurden in die Untersuchungen eingeschlossen. Die Diagnose wurde im Rahmen einer Polygrafie bzw. Polysomnografie über das Schlaflabor der Klinik und Poliklinik für Hals-Nasen-Ohrenheilkunde gestellt. Als Kontrollgruppe dienten Patienten mit primärem Schnarchen ohne Obstruktionen und gesunde Probanden. Neben klinischen Parametern wurden verschiedene Landmarken sonografisch evaluiert (u.a. Distanz Aa. linguales, horizontale und vertikale Zungendicke, Dicke der Pharynxwand).

Resultate In jede der fünf Gruppen (leicht-, mittel- und schwergradige OSA, primäres Schnarchen, gesunde Probanden) konnten 25 Patienten eingeschlossen werden. Die Auswertung der klinischen Parameter ergab signifikante Unterschiede hinsichtlich des Body Mass Index $(p<0,05)$ und des Halsumfanges $(p<0,05)$. Die Auswertung der sonografischen Parameter zeigte signifikant höhere Werte mit zunehmenden Schweregrad der OSA hinsichtlich Distanz zwischen den Aa. linguales $(p<0,05)$ sowie der horizontalen und vertikalen Dicke der Zunge $(p<0,05)$. Diese Messwerte zeigten ebenfalls signifikante Korrelationen mit der Anzahl der nächtlichen Apnoen und Hypopnoen.

Schlussfolgerung Mithilfe der sonografischen Beurteilung von für die obstruktive Schlafapnoe relevanter Landmarken konnten Korrelationen zwischen anatomischen Messwerten und dem Schweregrad der obstruktiven Schlafapnoe festgestellt werden. In weiteren Untersuchungen sollen diese Ergebnisse einen weiteren Einblick in die Pathogenese der obstruktiven Schlafapnoe geben und eine zielgerichtetere Therapie ermöglichen.

\section{05.03 Transoraler Ultraschall in der Diagnose von obstruktiven Erkrankungen der Speicheldrüsen}

Autoren Schapher $\mathrm{M}^{1}$, Goncalves $\mathrm{M}^{1}$, Mantsopoulos $\mathrm{K}^{1}$, Iro $\mathrm{H}^{1}$, Koch $\mathrm{M}^{1}$ Institut 1 Hals-Nasen-Ohrenklinik, Kopf- und Halschirurgie, Universität Erlangen-Nürnberg DOI 10.1055/s-0038-1670423

Einführung Wir beschreiben die Anwendung des transoralen Ultraschalls im Fachbereich der Hals-Nasen-Ohrenheilkunde zur Diagnostik und Differenzierung von obstruktiven Speicheldrüsenerkrankungen. Hervorgehoben werden sollen hierbei die Verfahren und Ergebnisse bei Sialolithiasis, Stenosen, Megaducts der Glandula parotidea und Raumforderungen im Bereich des Mundbodens. Die neuen Ergebnisse des transoralen Ultraschalls werden mit der bewährten transzervikalen bzw. transfazialen Ultraschalldiagnostik verglichen. Material und Methoden 71 Patienten, die sich aufgrund von obstruktiven Speicheldrüsen-erkrankungen (Glandula parotidea, Glandula submandibularis, Glandula sublingualis) in unserer Abteilung von Juli 2017 bis April 2018 vorstellten, wurden zusätzlich zu den diagnostischen Routinemaßnahmen und transzervikalem bzw. transfazialem Ultraschall mithilfe eines transoralen Ultraschallkopfes und einem high-end-Ultraschallgerät untersucht. Die Diagnosen aller an dieser Studie beteiligten Patienten wurden durch transzervikalen UItraschall, Speichelgangsendoskopie, chirurgische Darstellung oder durch pathohistologische Aufarbeitung bestätigt.

Ergebnisse In allen dargestellten Fragestellungen lieferte das transorale Verfahren detailliertere Abbildungen als der bewährte transzervikale oder transfaziale Ultraschall und in vielen Fällen therapieentscheidende zusätzliche Informationen, welche so bislang sonografisch nicht oder nur bedingt gewonnen werden konnten. Bei Sialolithiasispatienten können bestimmte Steinlokalisationen deutlich präziser oder überhaupt erst sonografisch diagnostiziert und selbst kleinste Konkremente sowie solche in unvorteilhaften Lagen erfasst werden. Bei anderen Fragestellungen werden anatomische Feinheiten detaillierter abgebildet, was die Effizienz des chirurgischen Vorgehens steigert. Im Falle von Megaducts der Ohrspeicheldrüse gelingt die präzise Darstellung der Feinarchitektur, was zusätzliche Erkenntnisse bei diesem Krankheitsbild ermöglicht.

Schlussfolgerung Die zusätzliche Anwendung eines transoralen Ultraschallverfahrens erhöht bei den beschriebenen Krankheitsbildern die diagnostische Genauigkeit deutlich, umgeht einige methodisch bedingte Nachteile der transzervikalen Anwendung und kann für die Diagnosesicherung obstruktiver Speicheldrüsenerkrankungen therapieentscheidend und von großer Hilfe sein. In Abhängigkeit von der Fragestellung ist der transorale Ultraschall dem transzervikalen bzw. transfazialen Verfahren überlegen.

\section{05.04 Die ARFI ${ }^{\odot}$-Technologie in der Diagnose und Verlaufskontrolle der glandulären Beteilung nach Kopf-Hals-Karzinom-Therapie}

Autoren Knopf $A^{1}$, Roth $A^{1}$, Schuhkraft J ${ }^{1}$, Hofauer $B^{1}$, Heiser $C^{1}$

Institut 1 Hals-Nasen-Ohren-Klinik und Poliklinik, Klinikum rechts der Isar, Technische Universität München

DOI 10.1055/s-0038-1670424

Hintergrund Die jeweiligen Therapieformen von Kopf-Hals-Karzinomen (HNSCC) führen in unterschiedlichem Ausmaß zu einer Schädigung der Speicheldrüsen, wobei bereits Strahlendosen von 20 Gy zu irreversiblem Schäden am Drüsengewebe führen.

Ziel Aktuelle Untersuchungen suggerieren, dass scherwellenelastografische Ultraschalltechniken bestens geeignet sind, um homogene Schäden an parenchymatösen Organen zu diagnostizieren und zu bewerten. Das Ziel dieser Untersuchung war es, den Wert der acoustic radiation force impulse (ARFI) imaging Technologie als Werkzeug für Diagnostik und Kontrolle der Speicheldrüsenaffektion nach HNSCC Therapie zu bewerten.

Material und Methoden Neunundsechzig HNSCC Patienten wurden in diese Untersuchung eingeschlossen. Alle Patienten hatten ihre Krebstherapie been- 
det und nahmen an der regulären Nachsorge teil. ARFI-Werte der ipsi- und kontralateralen Gll. parotes et submandibulares wurden nach einem standardisierten Protokoll erhoben und mit denen einer gesunden Kontrollgruppe verglichen. Nach 2 Monaten Behandlung mit einem liposomalen Speichelersatzmittel wurde eine erneute ARFI-Untersuchung durchgeführt.

Ergebnisse und Schlussfolgerung Ipsi- und kontralaterale Speicheldrüsen zeigen nach HNSCC Therapie signifikant erhöhte Scherwellenwerte; hierbei vor allem die ipsilaterale Glandula parotis. Hier lässt sich auch eine Hierachie der Schädigung OP < OP + CRT < pCRT mittels ARFI nachvollziehen. Durch liposomale Therapie kann bei den Drüsen und insbesondere bei der ipsilateralen Glandula parotis eine Besserung erzielt werden, wenngleich kein gesundes Niveau erreicht wird.

\section{05.05 Hochauflösende Sonografie des extratemporalen $\mathrm{N}$. facialis}

Autoren Wegscheider $\mathrm{H}^{1}$, Volk $\mathrm{GF}^{2}$, Moriggl $\mathrm{B}^{1}$, Guntinas-Lichius $\mathrm{O}^{2}$ Institut 1 Division of Clinical and Functional Anatomy, Department of Anatomy, Histology and Embryology, Medical University of Innsbruck (MUI), Innsbruck; 2 Department of Otorhinolaryngology, Facial-Nerve-Center Jena, Jena University Hospital, Jena

DOI 10.1055/s-0038-1670425

Einleitung Die technischen Fortschritte in der Sonografie ermöglichen inzwischen auch die Visualisierung von kleinsten peripheren Nerven. Die Darstellung von Teilen des Nervus facialis (NF) ist schon mehrfach versucht worden, bisher aber nicht vollständig wissenschaftlich belegt. Diese Studie beschreibt die erste systematische Auswertung des gesamten extratemporalen Verlaufs des NF.

Methoden Acht Halbköpfe von 4 Körperspendern wurden mittels Hochfrequenzultraschall am Punkt ihrer optimalen Visualisierbarkeit mit zwei linearen $(13 \mathrm{MHz}$ und $22 \mathrm{MHz})$ und einem konvexen Schallkopf $(6,6 \mathrm{MHz})$ untersucht, um den extratemporalen Verlauf des NF nachzuweisen: der Hauptstamm nach Austritt aus dem Foramen stylomastoideum, der Plexus parotideus in der Glandula parotis, sowie die peripheren Endäste in den M. orbicularis oculi und den M. zygomaticus major. Anschließend erfolgte eine Ultraschall-gezielte Injektion einer geeigneten (rasch aushärtenden), selbst hergestellten Farblösung $(0,01 \mathrm{ml})$ an die entsprechenden Anteile/Äste des NF. Zur Erfolgskontrolle wurden die Nerven abschließend präparatorisch freigelegt. Ergänzend stellten wir die Gabelung des NF („Dipus“) an 4 Seiten von 2 gesunden Probanden dar.

Ergebnisse Der Hauptstamm des NF wurde in 6/8 Fällen direkt gefärbt, in den restliche 2 Fällen lag das Farbdepot unmittelbar am Nerven. Der Plexus Parotideus konnte in 8/8 Fällen direkt gefärbt werden. Die Äste, die den Musculus orbicularis oculi innervieren, wurden in $7 / 7$ Fällen getroffen und die Äste zum M. zygomaticus major in 6/7 Halbköpfen (nachdem eine Halbseite wegen unzureichender Bildqualität ausgeschlossen wurde). Die Gabelung des NF konnte oberflächlich der Vena retromandibularis an beiden gesunden Probanden beidseits klar dargestellt werden.

Schlussfolgerungen Durch hochauflösende Sonografie können die verschiedenen Teile des extratemporalen Verlaufes des NF, einschließlich des Hauptstamms direkt nach seinem Austritt aus dem Foramen stylomastoideum, genau und reproduzierbar identifiziert werden. Die hochauflösende Sonografie des NF stellt unserer Meinung nach eine vielversprechende Methode für die klinische Anwendung (z. B. in der Gesichtschirurgie oder vor Operationen der Gl. parotis) dar.

\section{05.06 Darstellung von mediastinalen Raumforderungen (MRF) in der B-Bild- (US)- und kontrastmittelunterstützten Sonografie (CEUS)}

Autoren Trenker $\mathrm{C}^{1}$, Dohse $\mathrm{M}^{1}$, Holland $\mathrm{A}^{2}$, Schulte $\mathrm{F}^{3}$, Görg $\mathrm{C}^{3}$ Institut 1 Klinik für Hämatologie; 2 Klinik für Pneumologie; 3 Interdisziplinäres Ultraschallzentrum/1 - 3 Zentrum für Innere Medizin,
Universitätsklinikum Gießen und Marburg, Standort Marburg und Philipps-

Universität Marburg

DOI 10.1055/s-0038-1670426

Fragestellung Darstellung von mediastinalen Raumforderungen (MRF) in der B-Bild- (US)- und kontrastmittelunterstützten Sonografie (CEUS).

Patienten/Methoden Retrospektive Analyse von $n=58$ Patienten mit MRF, welche im Zeitraum von Oktober 2005 bis Februar 2018 mittels US und CEUS untersucht wurden. Eine histologische Diagnosesicherung erfolgte in allen Fällen ( $n=44(75,9 \%)$ direkt aus der MRF, $n=14(24,1 \%)$ an anderen Manifestationsorten bei metastasieren Tumorleiden oder Systemerkrankung). In $n=28$ $(51,9 \%)$ konnte die histologische Sicherung mittels sonografisch gesteuerter Stanzbiopsie erfolgen.

Ergebnisse $N=54(93,1 \%)$ der MRF waren maligne (m) (Lymphom $n=36^{*}$, Metastase $n=11$, Thymom $n=2$, Teratom $n=2$, Sarkom $n=2$, Seminom $n=1)$. In $n=4(6,9 \%)$ Fällen lag eine benigne (b) Histologie vor (Schilddrüsengewebe $n=2$, Thymusrest $n=1$, Ganglioneurinom $n=1)$. Im US waren $n=53$ $(91,4 \% ;(48=\mathrm{m}, 5=$ b)) echoarm, $\mathrm{n}=5(8,6 \% ;(5=\mathrm{m}, 0=\mathrm{b}))$ echoreich. In der CEUS zeigten die Läsionen ein $n=35$ arterielles Isoenhancement $(60,3 \%$; $(33=\mathrm{m}, 2=\mathrm{b})$. Ein arterielles Hypoenhancement hatten $\mathrm{n}=21 \quad(36,2 \%$; $(20=m, 1=b))$, kein Enhancement zeigten $n=2(3,5 \% ; \quad(1=m, 1=b)$ der MRF. In der Parenchymphase konnte ein Isoenhancement in $n=1 \quad(1,7 \%$; $(1=m, 0=b))$ Fällen, ein Hypoenhancement in $n=54$ (93,1\%; ( 51 = maligne, $3=$ benigne $)$ ) und ein fehlendes Enhancement bei $n=3 \operatorname{MRF}(5,2 \% ;(2=m$, $1=$ b) beobachtet werden.

Schlussfolgerung Die Beurteilung und histologische Sicherung MRF ist sonografisch häufig möglich. MRF waren überwiegend maligner Genese, wobei die malignen Lymphome die häufigste Entität stellten. B-Bildsonografisch dominiert eine echoarme Darstellung der MRF in 91,4\%. In der CEUS zeigen die mesenterialen Tumorformationen ein variables arterielles Enhancement, gefolgt von einem parenchymalem Hypoenhancement.

${ }^{*} \mathrm{~N}=19$ der Lymphome wurden im Rahmen eines Abstracts auf dem Ultraschall Dreiländertreffen 2008 präsentiert: A Holland 1, A Peil-Grun 2, C Görg 2; Ultraschall in Med 2008; 29 - V162 DOI: 10.1055/s-0028-1085896: Sonomorphologie maligner mediastinaler Lymphome im B-Bild und in der kontrastunterstützten Sonografie.

\section{05.07 Klinische Relevanz der quantitativen Muskelsonografie bei Patienten mit Fazialisparese}

Autoren Misikire $\mathrm{W}^{1}$, Puls $\mathrm{W}^{1}$, Thielker J ${ }^{1}$, Jarvis $\mathrm{JC}^{2}$, Guntinas-Lichius $\mathrm{O}^{1}$, Korten $\mathrm{S}^{1}$, Volk GF

Institut 1 Klinik und Poliklinik für Hals-, Nasen- und Ohrenheilkunde und Fazialis-Nerv-Zentrum Jena, Universitätsklinikum Jena; 2 School of Sport and Exercise Sciences, Liverpool John Moores University

DOI 10.1055/s-0038-1670427

Einführung Bei der Fazialisparese, der häufigsten Hirnnervenläsion, ist der Status der mimischen Muskulatur oft therapieentscheidend. Sonografisch können Veränderungen schon länger dank standardisierter Untersuchungsund Auswertungsprotokolle in Kohorten nachgewiesen werden, die Anwendung auf individueller Ebene zur Verlaufsdokumentation und um Therapieentscheidungen zu unterstützen, ist aber noch unüblich.

Methoden Die Aufnahmen der mimischen Muskeln wurden nach dem Sonografieprotokoll (Alfen et al. 2013, Volk et al. 2012) angefertigt und mit den entsprechenden Referenzwerten der Muskelgrößen (Volk et al. 2014) verglichen. Es wurde das Philipps Ultraschallgerät HD11 XE mit zwei unterschiedlichen Linearschallköpfen (12 und 15Mhz) verwendet. Von 7 mimischen Muskeln (M. frontalis, M. orbicularis oculi, M. zygomaticus major, M. orbicularis oris, M. depressor anguli oris, M. depressor labii, M. mentalis) und zwei Kaumuskeln als Referenz (M. temporalis und M. masseter) wurden UltraschallVideoaufnahme in Ruhe und in willkürlicher Kontraktion aufgenommen. Es erfolgt die Bestimmung der zwei Zustände durch den Untersucher. Nach einem detaillierten Segmentierungsprotokoll, in dem auf jeden einzelnen Mus- 
kel eingegangen wird, erfolgt die Quantifizierung der Muskelquerschnittsflächen durch ein MATLAB-Programm. Für jeden manuell eingezeichneten ROI werden automatisch die Fläche, der Längs- und Querdurchmesser und die Verteilung der Grauwerte ermittelt.

Resultat Dank der standardisierten Aufnahmen und des verbesserten Segmentierungsprotokolls ist die Quantifizierung der mimischen Muskulatur inzwischen so präzise, dass der Zeitverlauf individueller Patienten dargestellt werden kann. An mehreren Beispielen zeigen wir die Muskelatrophie nach Denervierung und typische Muskelveränderungen bei Reinnervation sowie unter Elektrotherapie. Das Ansprechen der Therapie und die Therapieentscheidungen können so auf individueller Ebene objektiviert werden.

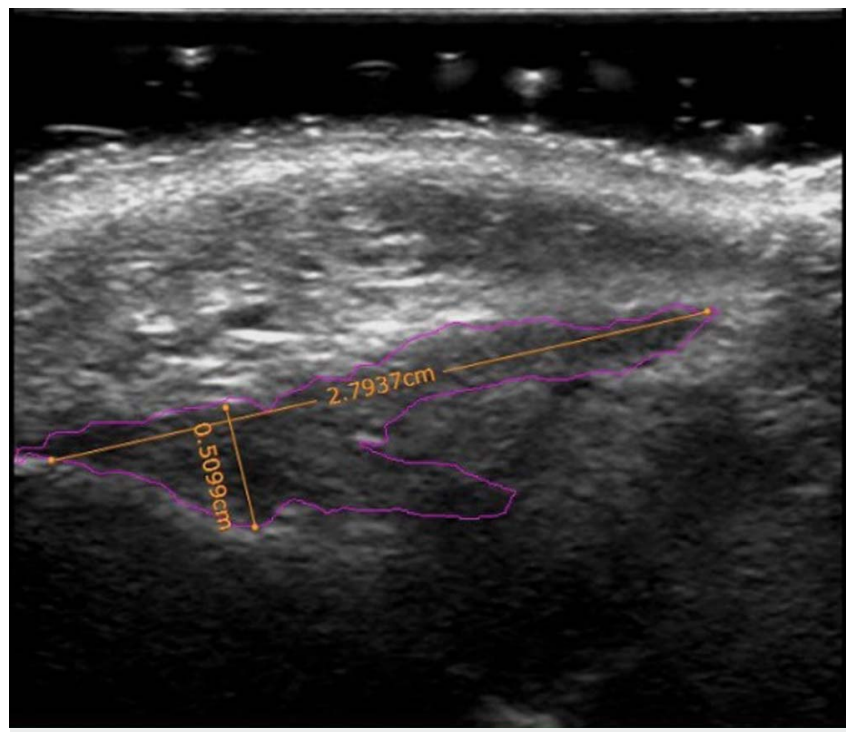

Abb. 1

Schlussfolgerung Durch die weiter verbesserte Vermessung und Auswertung können erstmals individuelle Atrophieverläufe präzise beschrieben werden. Darauf fußend können individuelle Therapieentscheidungen getroffen werden wie beispielsweise die Fortsetzung physikalischer Maßnahmen bei Nachweis des Therapieerfolges oder die Möglichkeit einer chirurgischen Reinnervation ohne zusätzlichen Muskeltransfer. So ist eine chirurgische Reinnervation nur sinnvoll, wenn ausreichend Muskelmasse vorhanden ist.

\section{Wi-Vo 06 Gynäkologie/Geburtshilfe III}

Do. 15.11. 14:00 - 15:30 Boston 3

\section{06.01 Accuracy of prenatal diagnosis of X-linked hypohidrotic ectodermal dysplasia by tooth germ sonography}

Authors Faschingbauer $\mathrm{F}^{1}$, Wohlfart $\mathrm{S}^{1}$, Goecke $\mathrm{T}^{2}$, Köninger $\mathrm{A}^{3}$, Stepan $\mathrm{H}^{4}$, Gallinat $\mathrm{R}^{5}$, Morris $\mathrm{S}^{6}$, Bücher $\mathrm{K}^{7}$, Clarke $A^{8}$, Beckmann $M W^{1}$, Schneider $\mathrm{H}^{1}$, Hammersen J

Institute 1 Center for Ectodermal Dysplasias, University Hospital Erlangen, Department of Prenatal Diagnostics and molecular Pediatrics, Erlangen, Germany; 2 Department of Gynecology and Obstetrics, RWTH Aachen, Germany; 3 Department of Gynecology and Obstetrics, University of Duisburg-Essen, Essen, Germany; 4 Department of Obstetrics, University Hospital Leipzig, Leipzig, Germany; 5 Frauenärztliche Gemeinschaftspraxis Günzburg-Ichenhausen, Günzburg, Germany; 6 Cardiff and Vale University Health Board, University Hospital of Wales, Cardiff, U.K.; 7 Department of Conservative Dentistry, Ludwig-Maximilians University Munich, Munich,
Germany; 8 Institute of Medical Genetics, School of Medicine, Cardiff University, Cardiff, U.K.

DOI 10.1055/s-0038-1670428

Objective X-linked hypohidrotic ectodermal dysplasia (XLHED), a developmental disorder characterized by malformation of hair, teeth, and sweat glands, results from defective ectodysplasin A1 (EDA1) caused by EDA mutations. Inability to sweat, the major problem of XLHED which can lead to lifethreatening hyperthermia, has been shown to be amenable to intrauterine therapy with recombinant EDA1. The aim of this retrospective study was to evaluate the diagnostic accuracy of tooth germ sonography to identify affected fetuses in pregnant women with EDA mutations.

Methods Tooth germ sonography was performed in 38 cases at 10 study sites between gestational weeks 18 and 28. XLHED was diagnosed if fewer than six tooth germs were detected in mandible and/or maxilla. In all subjects, diagnoses were verified postnatally by EDA sequencing and/or clinical findings (standardized clinical assessments of hair, sweating, and dentition; orthopantomograms). Estimated weights of 12 affected male fetuses and postnatal weight gain of 12 boys with XLHED were assessed using appropriate growth charts.

Results In 19 of 38 sonografic examinations of 23 male and 13 female fetuses, a prenatal diagnosis of XLHED was made. The diagnosis proved to be correct in 37 cases; one affected male fetus was missed. Specificity and positive predictive value were both $100 \%$. Tooth counting by clinical assessment corresponded well with radiografic findings. We observed no weight deficits of subjects with XLHED in utero but occasionally during infancy. Conclusions Tooth germ sonography is highly specific and reliable in establishing a prenatal diagnosis of XLHED.

\section{06.02 Adrenal Gland size in growth restricted fetuses}

Authors Oelmeier de Murcia $\mathrm{K}^{1}$, Heese $\mathrm{S}^{1}$, Hammer $\mathrm{K}^{1}$, Möllers $\mathrm{M}^{1}$, Köster $H^{1}$, Falkenberg $M^{1}$, Eveslage $M^{1}$, Braun $]^{1}$, Klockenbusch $W^{1}$, Schmitz $\mathrm{R}^{1}$

Institute 1 Geburtshilfe, Universitätsklinik Münster

DOI 10.1055/s-0038-1670429

Objective To compare the adrenal gland size of growth restricted (FGR) and normal control fetuses.

Study Design In this prospective study the adrenal gland size of 63 FGR fetuses and 343 normal controls was measured between 20 and 41 weeks of gestation. The total width and the medulla width were measured in a new standardized transversal plane. The cortex width and a calculated ratio of the total and medulla width (adrenal gland ratio) were compared between both groups.

Results The mean cortex width and the adrenal gland ratio in FGR fetuses were higher in comparison to the controls ( $<<0.001 ; p=0.036$; respectively). The cortex width correlated positively with the gestational age (control group: $\mathrm{p}<0.001$; FGR group: $\mathrm{p}=0.089$ ) whilst the adrenal gland ratio showed no association with the gestational age (control group: $p=0.153$; FGR group: $\mathrm{p}=0.314)$.

Conclusion The adrenal gland cortex width and the adrenal gland ratio were increased in FGR fetuses compared to normal fetuses.

\section{06.03 Myokardiale Funktion bei Feten mit Lower Urinary Tract Obstruction}

Autoren Graupner $\mathrm{O}^{1}$, Enzensberger $\mathrm{C}^{2}$, Götte $\mathrm{M}^{2}$, Wolter $\mathrm{A}^{2}$, Müller $\mathrm{V}^{2}$, Weber $\mathrm{S}^{3}$, Axt-Fliedner $\mathrm{R}^{2}$

Institut 1 Frauenklinik und Poliklinik, Klinikum rechts der Isar, Technische Universität München; 2 Klinik für Frauenheilkunde und Geburtshilfe, Abteilung für Pränatalmedizin, Universitätsklinikum Gießen; 3 Klinik für 
Kindernephrologie und Transplantationsnephrologie, Universitätsklinikum Marburg

DOI 10.1055/s-0038-1670430

Einführung Bei Feten mit Lower Urinary Tract Obstruction (LUTO) kommt es aufgrund von Nierenparenchym-Veränderungen zur Aktivierung des ReninAngiotensin-Aldosteron (RAAS) Systems mit potentiellem kardialen Remodeling. Dies kann Auswirkungen auf die myokardiale Funktion (MF) des Feten haben. Ziel der Studie ist es, mögliche Unterschiede in der MF bei LUTO-Feten im Vergleich zu gesunden Kontrollen während der Schwangerschaft aufzuzeigen.

Material und Methoden Es handelt sich um eine retrospektive Studie bestehend aus 23 LUTO-Feten und 37 gesunden Kontrollfeten. Das Schwangerschaftsalter war in beiden Gruppen gleichmäßig verteilt. Zum Zeitpunkt der Untersuchung hatten alle LUTO-Feten eine intrauterine Therapie erhalten. Die systolische Trikuspidal- (TAPSE) und Mitralklappenringauslenkung (MAPSE) wurde mittels M-Mode bestimmt. Die Messung der myokardialen Geschwindigkeiten und Zeitintervalle erfolgte mithilfe des gepulsten Gewebedopplers (PW-TDI). Die E'/A'-Ratio als Parameter der ventrikulären Relaxationsfähigkeit sowie der Myokardiale Performance Index (MPI') wurden für beide Ventrikel bestimmt. Zudem wurden laborchemische Parameter der fetalen Urinanalyse erfasst und mit den myokardialen Funktionsparametern korreliert.

Resultate Bei den LUTO-Feten zeigte sich eine signifikant niedrigere MAPSE $(4,2 \mathrm{~mm}$ vs. $5,5 \mathrm{~mm}, \mathrm{p}=0,002)$. Auch die TAPSE zeigte sich bei LUTO-Feten erniedrigt, allerdings ohne statistische Signifikanz $(5,9 \mathrm{~mm}$ vs. $6,6 \mathrm{~mm}$, $p=0,116)$. Für die rechtsventrikuläre (RV) E'/A'-Ratio ergaben sich signifikant niedrigere Werte im LUTO-Kollektiv verglichen mit den gesunden Kontrollen $(0,56$ vs. $0,62, p=0,018)$. Ebenso konnte ein signifikant niedriger RV MPI' bei den LUTO-Feten beobachtet werden (0,52 vs. 0,61, $p=0,013)$. Die Korrelation der MF Indizes mit den Parametern der fetalen Urinanalyse zeigte eine signifikant positive Korrelation zwischen der RV E'/A'-Ratio und $\beta$-2-Mikroglobulin $(r=0,56, p=0,01)$ sowie $\alpha-1$-Mikroglobulin $(r=0,65, p=0,005)$.

Schlussfolgerung Es konnten Unterschiede in der MF bei LUTO-Feten im Vergleich zu gesunden Kontrollen nachgewiesen werden. Dies kann als Zeichen eines intrauterin stattfindenden kardialen Remodeling verstanden werden. Weiterhin konnte ein Zusammenhang zwischen der ventrikulären Relaxation und der renalen Proteinsekretion beobachtet werden. Eine Bestätigung dieser ersten Resultate sollte anhand von prospektiven Studien mit größeren Fallzahlen erfolgen.

\section{06.04 Outcome von 1376 Feten mit pränataldiagnostisch (zu) kurzem Femur}

Autoren Friebe-Hoffmann $U^{1}$, Dobravsky $L^{1}$, Friedl TWP ${ }^{1}$, Janni $W^{1}$, Knippel $\mathrm{A}^{2}$, Siegmann $\mathrm{HJ}^{2}$, Kozlowski $\mathrm{P}^{2}$

Institut 1 Universitätsfrauenklinik, Ulm; 2 praenatal.de, Düsseldorf DOI 10.1055/s-0038-1670431

Einführung Der (zu) kurze fetale Femur in der Pränataldiagnostik (PND) kann Indiz sein für eine intrauterine Wachstumsretardierung, ein genetisch bedingt kleines Kind, für assoziierte fetale Fehlbildungen sowie ein adverses fetales Outcome.

Patienten/Methoden 1376 zwischen 1997 - 2014 pränatal untersuchten Feten, deren Femura sich im Zweitsemesterscreening (18+0 bis $21+6$ SSW) unterhalb der 5. Längenperzentile befanden (Verburg et al., Ultrasound Obstet Gynecol 2008; 31: 388 -396) wurden einer beschreibenden retrospektiven Single-Center-Analyse hinsichtlich Fehlbildungsraten, Schwangerschaftsverlauf und perinatalem Outcome unterzogen.

Resultate Bei insgesamt 822 (59,7\%) Feten lag ein isolierter kurzer Femur im Ultraschallbefund vor, während bei 554 (40,3\%) der Feten pränataldiagnostisch weitere Auffälligkeiten festgestellt wurden. 323 (58,3\%) der 554 Feten wiesen schwerwiegende Fehlbildungen in einem oder mehreren Bereichen auf: 153 (27,6\%) Gefäß- und/oder Herzauffälligkeiten, 35 (6,3\%) Gastrointestinaltrakt- oder Thoraxabnormalitäten, 30 (5,4\%) Teratome/Zysten, 27 (4,9\%)
Gehirn- oder Kopfentwicklungsauffälligkeiten, 5 (0,9\%) Nierenanomalien, 67 (12,1\%) Chromosomenaberrationen, und 118 (21,3\%) weitere Auffälligkeiten. Eine Trisomie 13, 18 bzw. 21 fand sich in 2, 13 bzw. 27 der Fälle. Feten mit kurzem Femur und assoziierten Anomalien wiesen eine deutlich niedrigere Lebendgeburtenrate auf als Feten mit einem isolierten kurzen Femur (78,5\% vs. 97,9\%, p<0,001); außerdem zeigte sich in ersterem Kollektiv eine höhere Rate an Frühgeburten $(33,5 \%$ vs. $8,1 \%, p<0,001)$ und small for gestational age (SGA) Babys (53,0\% vs. 25,5\%, $p<0,001)$.

Schlussfolgerung Die Diagnose des kurzen Femurs im Rahmen der biometrischen Messung sollte ein erweitertes Organscreening nach sich ziehen und bei assoziierten Auffälligkeiten eine invasive Diagnostik angeboten werden.

\section{06.05 Der sFlt-1/PIGF-Quotient als Prädiktor für das Auftreten von Präeklampsie-assoziierten Komplikationen}

Autoren Kreße-Chludek $\mathrm{P}^{1}$, Pullankavumkal J ${ }^{2}$, Klöckner $\mathrm{N}^{2}$, Henrich $\mathrm{W}^{1}$, Verlohren $\mathrm{S}^{1}$

Institut 1 Klinik für Geburtsmedizin; 2 Klinik für Gynäkologie/1 - 2 Charité Universitätsmedizin Berlin

DOI 10.1055/s-0038-1670432

Einführung Ziel dieser Untersuchung ist die Evaluation der Vorhersagekraft eines erhöhten sFlt-1/PIGF-Quotienten nach 34+0 SSW für das Auftreten Präeklampsie-bedingter Komplikationen mit besonderem Blick auf Small for Gestational Age (SGA) Neugeborene.

Methoden 176 Schwangere (erste sFlt-1/PIGF Messung bei klinischem Verdacht auf Präeklampsie nach 336/7 SSW) wurden retrospektiv untersucht. Als Präeklampsie-assoziierte mütterliche und fetale Komplikationen lagen in dieser Kohorte das Auftreten hypertensiver Schwangerschaftserkrankungen (HPD), vorzeitige Plazentalösung, Infant Respiratory Distress Syndrome (IRDS) und SGA Neugeborene vor. SGA wurde definiert als Geburtsgewicht unterhalb der 10. Perzentile. Der Zusammenhang des sFlt-1/PIGF-Quotienten und aufgetretenen Komplikationen, bzw. SGA gesondert, wurde mittels Mann-Whitney-U-Test und binär-logistische Regressionsanalyse überprüft. Ein Trennwert zur Vorhersage von maternalen und fetalen Komplikationen wurde mittels ROC-Kurvenanalyse und Berechnung des Youden-Index ermittelt.

Resultate Bei 100 Schwangeren (56,8\%) trat eine HPD auf, in 2 Fällen $(1,1 \%)$ kam es zu einer vorzeitigen Plazentalösung, 14 Säuglinge (8\%) litten am IRDS und 28 (15,9\%) waren SGA. Der mediane sFlt-1/PIGF-Quotient 77,0 (IQR 27,0 - 161,5) in der Gruppe mit Komplikationen war gegenüber dem Quotient der Gruppe ohne Komplikationen mit 16,0 (IQR 6,0-58,0) signifikant erhöht $(p<0,001)$. Ebenso war der mediane Quotient beim Auftreten eines SGA signifikant höher (SGA 118,5 (IQR 58,8-231,5) vs. kein SGA 39,0 (10,391,8); $p<0,001)$. Mittels Binär-logistischer Regressionsanalyse konnte der sFlt-1/PIGF-Quotient als signifikanter Prädiktor auftretender Komplikationen und eines SGAs identifiziert werden (Komplikationen: $r=1,019$; 95\% Konfidenzintervall (KI): 1,011-1,028; $\mathrm{p}<0,001$; SGA: $r=1,004 ; 95 \% \mathrm{KI}: 1,001-$ 1,007; $p=0,003)$. ROC-Kurven-Analyse und Berechnung des Youden-Index ergaben einen optimalen Trennwert zur Vorhersage von Komplikationen von 84,5 (Area under the curve 0,76; $p<0,001$; Sensitivität 47,9\%; Spezifität 94,99\%).

Schlussfolgerung Wir konnten einen signifikanten Zusammenhang zwischen einem erhöhten sFlt-1/PIGF-Quotient und dem Auftreten Präeklampsie-bedingter Komplikationen, insbesondere SGA, feststellen. Der Trennwert 84,5 erwies sich, vor allem aufgrund seiner hohen Spezifität, als sinnvoller prädiktiver Marker zum Ausschluss Präeklampsie-bedingter Komplikationen in der Spätschwangerschaft. 


\section{06.06 Cerebro-plazentare Ratio und das Risiko für operative Entbindung am Termin}

Autoren Ortiz $\mathrm{JU}^{1}$, Ostermayer $\mathrm{E}^{1}$, Graupner $\mathrm{O}^{1}$, Kuschel $\mathrm{B}^{1}$, Lobmaier SM ${ }^{1}$ Institut 1 Sektion Geburtshilfe und Perinatalmedizin, Technische Universität München

DOI 10.1055/s-0038-1670433

Einführung Das Hauptziel dieser Studie war die Auswertung der Assoziation zwischen der fetalen cerebro-plazentaren Ratio (CPR) und der Notwendigkeit einer operativen Entbindung (Vakuum, Forzeps, sekundäre bzw. Notsectio) wegen intrapartalem fetalen Distress. Als sekundäres Ziel wurde die Aussagekraft der CPR analysiert.

Material und Methoden Dies war eine retrospektive Studie von Schwangeren mit Einlingsschwangerschaften, die zwischen Januar 2010 und Dezember 2017 und zwischen $37+0$ und 41+6 SSW in unserem Klinikum entbunden haben. Die Dopplermessungen wurden innerhalb von 14 Tagen vor der Entbindung durchgeführt. Die CPR wurde als Quotient zwischen den Pulsatilitätsindizes der Arteria cerebri media und Umbilikalarterie berechnet. Die Schwangerschaften wurden nach dem Neugeborenengewicht (small-for-gestational age $(S G A)<10$. Perzentile $(P)$, appropriate-for-gestational age (AGA) 10. -90. P.) und der CPR (<10. P., $\geq 10$. P.) in vier Gruppen eingeteilt. Als intrapartaler fetaler Distress wurde ein persistierendes pathologisches CTG nach den FIGO-Kriterien definiert.

Resultate 3187 Patientinnen erfüllten die Kriterien und wurden wie folgt eingeteilt: SGA mit CPR < 10. P. $(n=130)$, SGA mit CPR $\geq 10$. P. $(n=348)$, AGA mit CPR < 10. P. $(n=356)$ und AGA mit CPR $\geq 10$. P. $(n=2353)$. Der Anteil an operativen Entbindungen aufgrund eines intrapartalen fetalen Distresses war signifikant höher bei Feten mit niedriger CPR sowohl im SGA- $(29,2 \%$ vs. $18,4 \%, p=0,010)$ als auch im AGA-Kollektiv $(18,3 \%$ vs. $12 \%, p=0,001)$. Die Aussagekraft der CPR < 10. P. bzgl. fetalen Distresses war niedrig in beiden Gruppen mit Detektionsraten von 19\% (AGA) bzw. 37\% (SGA), falsch-positivRaten von 12\% (AGA) bzw. 24\% (SGA) und positivem Vorhersagewert von 18\% (AGA) bzw. 29\% (SGA).

Schlussfolgerung Feten mit niedriger CPR haben ein höheres Risiko für operative Entbindungen am Termin wegen fetalem Distress unabhängig vom Gewicht. Allerdings ist die Vorhersagekapazität der CPR niedrig.

\section{06.07 A systolic shoulder in fetal middle cerebral artery Doppler waveforms may indicate fetal pulse wave reflection and transmission to cerebral circulation - a contribution to fetal circulatory physiology}

Authors Gonser $\mathrm{M}^{1}$, Tavares de Sousa $\mathrm{M}^{2}$, Klee $\mathrm{A}^{1}$, Hecher $\mathrm{K}^{2}$ Institute 1 Department of Obstetrics and Prenatal Medicine, Helios-HSK Kliniken, Wiesbaden; 2 Department of Obstetrics and Fetal Medicine, University Medical Center Hamburg-Eppendorf (UKE)

DOI 10.1055/s-0038-1670434

Introduction A systolic shoulder may appear in fetal middle cerebral artery (MCA) Doppler waveforms during the basically monotone decelerative phase. This waveform feature is observed occasionally, also in published MCA Doppler waveforms. Recently we found that a 2nd mid-systolic peak in MCA Doppler in fetuses with severe anemia may indicate increased pulse wave (PW) reflection in the lower body and secondary transmission to cerebral circulation. We assume that a systolic shoulder may represent a minor form of a 2 nd midsystolic peak in less reflective conditions.

Patients and Methods Arterial PW reflection seems to originate mainly from the pelvic region (Nichols 2011) with retrograde transmission to cerebral circulation and transient flow augmentation on arrival at the Doppler sampling site (Heffernan 2013, Liang 2015). In this model the time interval dt between onset of MCA Doppler waveform and onset of the systolic shoulder corresponds to the 2-way travel time (2wTT) of the PW (Fig. 1), travelling with velocity $c$ along the distance $L$ to the pelvic region and back:
$2 \mathrm{wTT}=2 \mathrm{~L} / \mathrm{c}$ (Nichols 2011), $\mathrm{L}=$ length (aorta + common iliac artery). To validate this model for fetal application we performed a preliminary search in our perinatal databases for fetuses, were MCA Doppler was performed for placental insufficiency. When a MCA shoulder was present, we compared observed intervals dt with predicted 2wTT, based on human fetal data on anatomical distance L (Szpinda 2008) and PW velocity c (Struijk 2011).

Results We identified 20 fetuses with IUGR and/or increased uteroplacental resistance and a systolic shoulder in MCA waveforms with adequate Doppler quality for dt measurements. Mean (SD) GA was 31 (3) wks and mean systolic shoulder timing dt was 96 (15) ms. Human fetal data adjusted to 31 wks GA yield: $P W$ travel distance $L=11.4 \mathrm{~cm}$, PW velocity $\mathrm{c}=271 \mathrm{~cm} / \mathrm{s}$, and thus we obtain $2 \mathrm{wTT}=2 \mathrm{~L} / \mathrm{c}=84 \mathrm{~ms}$, indicating fairly good agreement between model prediction and observation within the range of temporal resolution of Doppler devices.

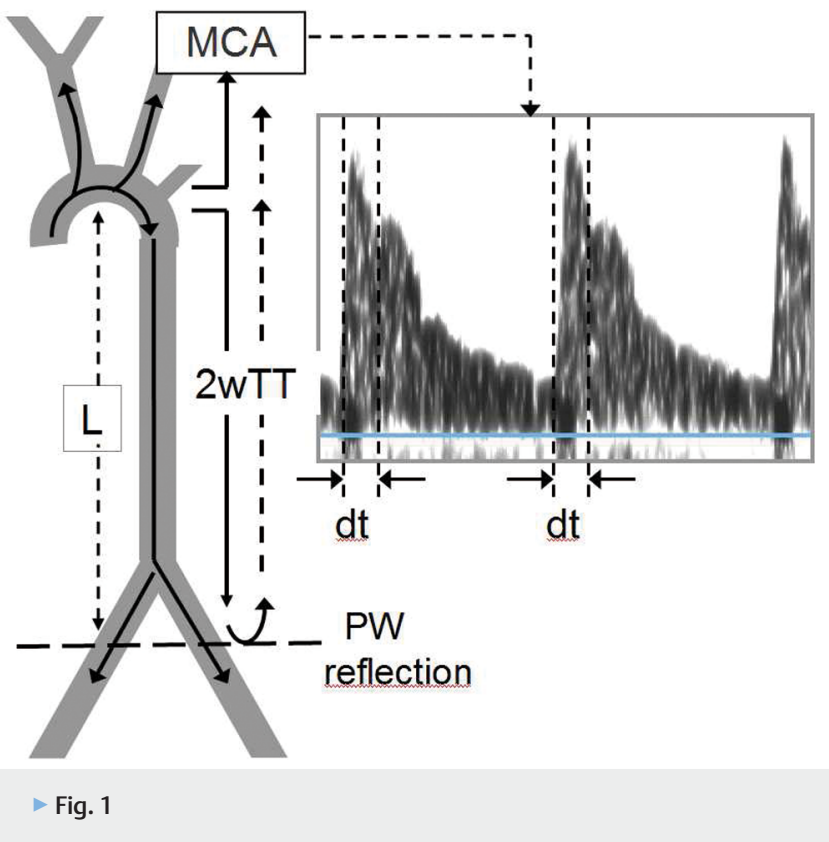

Conclusion The appearance of a systolic shoulder in fetal MCA Doppler shows evidence of PW reflection in the lower body of the human fetus. According to the fetal circulatory repertoir up to circulatory centralization, changing vascular tone and peripheral vasoconstriction are supposed to modulate PW reflection with varying impact on MCA systolic waveform, ranging from a hardly dicernible systolic shoulder to a 2nd systolic peak, when PW reflection is considerably increased.

Wi-Vo 07 Kontrastmittel

Fr. 16.11. 08:30 - 10:00 Shanghai 2

V 07.01 Comparison of the diagnostic accuracy of contrast - enhanced ultrasound (CEUS) within 5 minutes and $C T / M R I$ for diagnosing vital tumor tissue after RFA/TACE in HCC and liver metastasis in a tertiary Swiss GI center

Authors Sawatzki $\mathrm{M}^{1}$, Hechelhammer $\mathrm{L}^{2}$, Semela $\mathrm{D}^{1}$, Brand $\mathrm{S}^{1}$ Institute 1 Klinik für Gastroenterologie/Hepatologie; 2 Klinik für Radiologie/

1 - 2 Kantonsspital St. Gallen

DOI 10.1055/s-0038-1670435

Background CEUS is accurate for follow-up after radiofrequency ablation (RFA) and transarterial chemoembolization (TACE) in HCC or liver metastasis. However, data are missing for CEUS in comparison with CT/MRI in clinical routine. Follow-up imaging according to modified RECIST is performed only 
by CT and MRI. Our aim was to assess the response of malignant focal liver lesions (FLL) after RFA or TACE by CEUS in comparison to CT/MRI in every day practice.

Methods The study was performed from $1 / 2011$ to $11 / 2013$ by three experienced gastroenterologists (at least level II of training according to EFSUMB) after RFA and/or TACE in comparison to CT/MRI (with contrast application). All patients were examined according the international guidelines with intravenous sulphur hexafluoride microbubbles as contrast agent. Representative still images and video clips were recorded over a time interval of 5 minutes. Intratumoral arterial enhancement in any of the three imaging modalities (CEUS, CT, MRI) was defined as presence of vital tumor tissue, while a wash-out phenomen reinforced the diagnosis of tumor tissue in CEUS. Standard of reference (SOR) was defined as concordant results of CEUS and CT/ MRI at follow-up.

Results In all 20 patients ( $\mathrm{HCC} n=17$; cholangiocarcinoma $n=1$; liver metastasis of colorectal carcinoma $n=2$ with age range: $43-86$ years), no complications occurred after CEUS. CEUS was compared with CT/MRI in 39 examinations after 30 interventions (RFA $n=13$; TACE $n=17$ ). The median follow-up period was 6.7 months. Imaging was performed after one month and thereafter every three months following RFA and/or TACE. In seven different cases, CEUS and CT/MRI could not demonstrate vital tumor tissue after RFA/TACE. $\mathrm{CT} / \mathrm{MRI}$ missed vital tumor tissue in 4 cases (10\%) found in CEUS, while CEUS alone did not diagnose vital tumor tissue in 3 cases (8\%). One false-positive MRI finding was detected by CEUS as reactive hypervascularisation and confirmed by MRI-follow-up. Sensitivity and specificity with predictive values are shown in table 1.

Tab. 1 Comparison of the diagnostic accuracy of CEUS and CT/MRI in 20 patients with a total of $n=39$ CEUS investigations in comparison to $n=39$ CT/MRI (mean follow-up period: 6.7 months)

\begin{tabular}{l|l|l|l|l|l|} 
& sensitivity & $\mathrm{npV}$ & specificity & ppV & accuracy \\
\hline CEUS & $80 \%$ & $88 \%$ & $100 \%$ & $100 \%$ & $92 \%$ \\
\hline CT/MRI & $75 \%$ & $85 \%$ & $96 \%$ & $92 \%$ & $87.5 \%$ \\
\hline
\end{tabular}

Conclusions In this study, CEUS had no side effects and demonstrated a high accuracy of $90 \%$ to diagnose vital tumor tissue tumor response after RFA/ TACE. The diagnostic accuracy of CEUS was not inferior to CT/MRI. Combination of CEUS with CT/MRI could avoid $20 \%$ of false negative results, thereby increasing the accuracy vital tumor detection. We discuss alternating CEUS with CT/MRI for the consecutive 3 month follow-up periods suggested by the modified RECIST.

\section{07.02 Interobserver-Variabilität von Kontrastmittelultraschall-(CEUS-) basierten standardisierten Diagnosealgorithmen für das hepatozelluläre Karzinom (HCC) bei Hochrisikopatienten}

Autoren Schellhaas $B^{1}$, Pfeifer $L^{1}$, Kielisch $C^{1}$, Goertz RS $^{1}$, Neurath $M F^{1}$, Strobel $D^{1}$

Institut 1 Medizinische Klinik 1, Universitätsklinikum Erlangen

DOI 10.1055/s-0038-1670436

Einleitung Das hepatozelluläre Karzinom (HCC) kann bei Hochrisikopatienten nicht-invasiv diagnostiziert werden, wenn ein typisches Kontrastmittel-Verhalten in kontrastverstärkter Bildgebung (Computertompgrafie, Magnetresonanztomografie oder Kontrastmittelultraschall =CEUS) vorliegt. Hepatozelluläre Karzinome stellen jedoch eine sehr heterogene Tumorentität dar; das „typische“ Kontrastmittelverhalten (arterielles Hyperenhancement, gefolgt von Hypoenhancement in der portalvenösen Phase oder Spätphase) findet sich nicht bei allen HCCs. Zur Verbesserung der diagnostischen Treffsicherheit und Standardisierung in der Befundung von CEUS bei HCC-Risikopatienten wurden deshalb CEUS-basierte Algorithmen entwickelt [Schellhaas B. et al. Ultraschall Med. 2016 Dec;37(6):627 - 634; Schellhaas B et al. Eur J Gastroenterol Hepatol. 2017 Sep;29(9):1036 - 1044; Piscaglia F. et al. Ultraschall Med.
2017 Mar 22]. Diese CEUS-basierten Diagnosealgorithmen sind bislang noch nicht klinisch validiert. Das Ziel der vorliegenden Pilotstudie war daher die Untersuchung der Interobserver-Variabilität zweier Kontrastmittelultraschall(CEUS-)-basierter Diagnosealgorithmen für die Diagnostik des hepatozellulären Karzinoms (HCC) bei Hochrisikopatienten im direkten Vergleich.

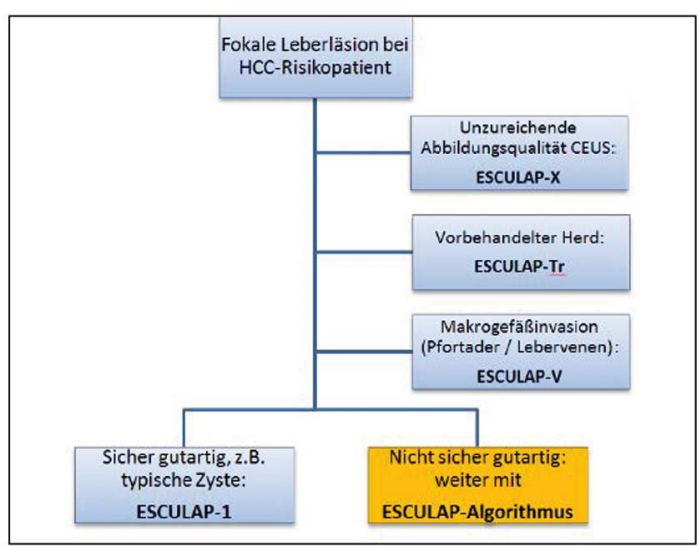

\begin{tabular}{|c|c|c|c|c|c|}
\hline \multicolumn{6}{|c|}{ ESCULAP-Algorithmus } \\
\hline & & \multicolumn{2}{|c|}{$\begin{array}{c}\text { Arterielle Phase: } \\
\text { Iso-/ Hypoenhancement }\end{array}$} & \multicolumn{2}{|c|}{$\begin{array}{l}\text { Arterielle Phase: } \\
\text { Hyperenhancement }{ }^{\# \#}\end{array}$} \\
\hline Durchmesser [mm] & & $<20$ & $\geq 20$ & $<20$ & $\geq 20$ \\
\hline \multirow{4}{*}{$\begin{array}{l}\text { Zusatzkriterien (ZK): } \\
\text { - } \text { Wash-out portalvenös / } \\
\text { Spätphase } \\
\text { - Subtotale Infiltration } \\
\text { eines Leberlappens } \\
\approx 2 \mathrm{ZK}\end{array}$} & $02 \mathrm{~K}$ & ESCULAP-2" & ESCULAP-3 & ESCULAP-3 & ESCULAP-4 \\
\hline & $1 \mathrm{ZK}$ & ESCULAP-3 & ESCULAP-4 & ESCULAP-4 & ESCULAP-4 \\
\hline & $\geq 2 \mathrm{zK}$ & & ESCULAP-4 & & ESCULAP-4 \\
\hline & \multicolumn{5}{|c|}{$\begin{array}{l}\text { "ESCULAP-1 für typische Zyste } \\
\text { "ESCULAP-C, wenn beide ICC-Kriterien erfüllt sind: } \\
\text { ICC-Kriterien: } \\
\text { - Frühes Wash-out < } 60 \text { Sekunden } \\
\text { " "rim-like" arterielles Hyperenhancement }\end{array}$} \\
\hline
\end{tabular}

Abb. 1

Methoden Fokale Leberläsionen bei 55 Hochrisikopatienten wurden unabhängig voneinander von drei verblindeten Untersuchern anhand beider CEUSAlgorithmen klassifiziert (ESCULAP = Erlanger Synopsis of Contrast-enhancedUltrasound for Liver lesion Assessment in Patients at Risk, Abb. 1; ACR-CEUSLI-RADS ${ }^{\circledR}$ v.2016 = American College of Radiology CEUS-Liver Imaging Reporting and Data System). Die Kategorisierung erfolgte nach Größe und Ultraschallkontrastmittelverhalten in arterieller, portalvenöser und Spätphase. Die Interobserver-Variabilität für Kontrastmittelverhalten und Kategorisierung sowie die diagnostische Genauigkeit für die definitive HCC-Diagnose wurden für beide Algorithmen verglichen. Histologie und CE-MRT dienten als Referenzstandard.

Ergebnisse 55 Patienten wurden eingeschlossen (männlich/weiblich =44/11; 90,9\% Leberzirrhose). Histologische Befunde waren in 39/55 Läsionen verfügbar (70,9\%). Der Referenzstandard ergab 48 HCCs, 2 ICCs, und 5 Non-HCCnon-ICC-Läsionen. Die Interobserver-Übereinstimmung war mäßig bis beachtlich für arterielles Hyperenhancement ( $(=0,53-0,67)$, und ausreichend bis mäßig für Washout $(\kappa=0,33-0,53)$. Die Interobserver-Variabilität war beachtlich für die ESCULAP-Kategorie $(\kappa=0,64-0,68)$, und ausreichend für die CEUSLI-RADS. 


\section{07.03 Interobserver- und Verfahrens-Übereinstimmung für standardisierte Diagnose-Algorithmen für das Heptozelluläre Karzinom (HCC) bei Hochrisikopatienten - CEUS-LI-RADS $^{\odot}$ versus MRT-LI-RADS ${ }^{\odot}$}

Autoren Schellhaas $B^{1}$, Hammon $M^{2}$, Strobel $D^{1}$, Pfeifer $L^{1}$, Kielisch $C^{1}$, Goertz $\mathrm{RS}^{1}$, Cavallaro $\mathrm{A}^{2}$, Janka $\mathrm{R}^{2}$, Neurath $\mathrm{MF}^{1}$, Uder $\mathrm{M}^{2}$, Seuss $\mathrm{H}^{2}$ Institut 1 Medizinische Klinik 1; 2 Radiologisches Institut/1-2

Universitätsklinikum Erlangen

DOI 10.1055/s-0038-1670437

Einleitung Standardisierte Befundungsalgorithmen wie LI-RADS (Liver Imaging Reporting and Data System) für CT und MRT sollen die diagnostische Treffsicherheit und Standardisierung in der Befundung HCC-suspekter Leberrundherde verbessern. Neu sind analoge Algorithmen für die Kontrastmittelsonografie (CEUS). Diese CEUS-basierten Diagnosealgorithmen sind bislang noch nicht klinisch validiert. Ziel der vorliegenden Pilotstudie war die Untersuchung der Interobserver-Variabilität des neu entwickelten CEUSbasierten Diagnosealgorithmus CEUS-LI-RADS im direkten Vergleich mit dem bereits etablierten MRT-LI-RADS.

Methoden CEUS- und MRT-Befunde fokaler Leberläsionen bei 50 HCC-Risikopatienten (mittleres Alter: 66,2 $\pm 11,8 ; 78 \%$ männlich) wurden retrospektiv durch jeweils zwei unabhängige, verblindete Observer mittels CEUS-LI-RADS und MRT-LI-RADS befundet. Die Interobserver-Übereinstimmung wurde nach Cohen's Kappa errechnet.

Ergebnisse Histologische Befunde waren für 36/50 Läsionen verfügbar (72\%). Es fanden sich 43 HCCs, 2 intrahepatische cholangiozelluläre Karzinome (ICCs), und 5 benigne Läsionen. Arterielles Hyperenhancement fand sich bei $37 / 50 / 38 / 50$ Läsionen $=74 \% / 78 \%$ (Observer $1 /$ Observer 2 ) für CEUS und $46 / 50 / 44 / 50$ Läsionen $=92 \% / 88 \%$ (Observer $1 /$ Observer 2 ) im MRT. Ein Auswaschen fand sich für CEUS seltener als im MRT (34/50/20/50 Läsionen $=68 \% / 40 \%$ versus $31 / 50 / 31 / 50$ Läsionen $=62 \% / 62 \%$ ). Das Interobserver-Agreement war mäßig für arterielles Hyperenhancement $(\kappa=0,511 / 0,565$ [CEUS/MRI]) und „Auswaschen“ ( $k=0,490 / 0,582$ [CEUS/ MRI]), ausreichend für die CEUS-LI-RADS-Kategorie $(\mathrm{k}=0,309)$, und beachtlich für die MRT-LI-RADS-Kategorie ( $k=0,609)$. Das Agreement zwischen beiden Verfahren war ausreichend für arterielles Hyperenhancement $(k=0,329)$ und gering bis ausreichend für „Auswaschen“ $(\mathrm{k}=0,202)$ und die LI-RADS-Kategorie $(\mathrm{k}=0,218)$.

Schlussfolgerungen Die Interobserver-Übereinstimmung ist für MRT-LI-RADS besser als für CEUS-LI-RADS. Hauptursache hierfür ist die geringere Übereinstimmung für "Auswaschen“ in CEUS, da HCCS mit alleinigem Hyperenhancement mit CEUS-LI-RADS als LR-4 klassifiziert werden.

\section{07.04 Contrast-enhanced ultrasound (CEUS) quantifies the perfusion within tibial non-unions and predicts the outcome of revision surgery}

Authors Krammer $\mathrm{D}^{1}$, Schmidmaier $\mathrm{G}^{1}$, Weber $\mathrm{MA}^{2,3}$, Doll $\rfloor^{1}$, Rehnitz $\mathrm{C}^{3}$, Fischer $C^{1}$

Institute 1 Center for Orthopedics, Trauma Surgery and Spinal Cord Injury; 2 Institute of Diagnostic and Interventional Radiology University Medical Center Rostock; 3 Diagnostic and Interventional Radiology/1,3 Heidelberg University Hospital

DOI 10.1055/s-0038-1670438

Introduction Vascularity is one of the determining factors of successful bone regeneration. This prospective study focused on quantifying the microperfusion of tibial non-unions with contrast-enhanced ultrasound (CEUS) and comparing it to the outcome.

Materials and Methods 36 patients with tibial fracture non-unions received a CEUS examination 12 weeks after revision surgery and the osseous microper- fusion was quantified. The osseous consolidation at a maximum of 24 months was assessed with standard $\mathrm{x}$-rays and computed tomography.

Results 28 out of 36 non-unions (77.8\%) showed consolidation, while 8 patients required further revision surgery. CEUS demonstrated significantly higher perfusion in consolidated vs. persistent non-unions for all quantification parameters (e.g.: Wash-in Perfusion Index (WiPI) p=0.036). The Receiver Operating Characteristics (ROC) analysis revealed a sensitivity/specificity of $82.1 \% / 75.0 \%$ with a WiPl cut-off at 19.9 a.u. for diagnosing persisting nonunions.

Discussion More than one year ahead of the final radiologic diagnostic examination, CEUS could predict eventual consolidation based on the osseous perfusion as soon as 12 weeks postoperatively. This information can be crucial for the decision making process of re-revision at an early stage.

\section{07.05 Perfusionsbeurteilung bei Nierentransplantaten: Machen digitale Dopplerverfahren den Kontrastmittelultraschall (CEUS) überflüssig?}

Autoren Putz FJ ${ }^{1}$, Banas $\mathrm{MC}^{1}$, Stroszczynski $\mathrm{C}^{2}$, Banas $\mathrm{B}^{1}$, Jung EM² Institut 1 Abteilung für Nephrologie; 2 Interdisziplinäres Ultraschallzentrum - Institut für Röntgendiagnostik/1 - 2 Universitätsklinikum Regensburg DOI 10.1055/s-0038-1670439

Hintergrund Zur Beurteilung von Nierentransplantaten ist der Ultraschall die Methode der Wahl in der Routinekontrolle und bei vermuteten Problemen. Neue, weniger winkelabhängige digitale Blutflussdarstellungen (z. B. B-Flow) benötigen kein Kontrastmittel und zeigen Blutgefäße in einer exzellenten Qualität. Allerdings sind diese nur bei oberflächlicher Organlage einsetzbar. Der Kontrastmittelultraschall (CEUS) ist allein in der Lage die Mikrozirkulation auf kapillarer Ebene dynamisch zu erfassen. Ziel der Studie ist die Anwendbarkeit und Aussagekraft unterschiedlicher Ultraschallmethoden bei Nierentransplantaten einzuschätzen und die Bildqualität unter Altagsbedingungen zu beurteilen.

Material und Methoden 50 Transplantatnieren von 41 Patienten wurden von einem erfahrenem Ultraschaller an einem high-end Ultraschallgerät mit BMode, Farbdoppler (FKDS), B-Flow, Kontrastmittel-verstärktem Ultraschall (CEUS) und Kontrastmittel-verstärktem B-Flow (ceB-Flow) untersucht. Der Kontrastmittelultraschall erfolgte in der low-MI-Technik nach Applikation von 0,5-1,5 ml Sulfurhexafluorid-Mikrobläschen. Zwei erfahrene Ultraschaller bewerteten klinische Aussagekraft, die Blutflussdarstellung, die Bildqualität und die maximale Eindringtiefe anhand von Bewertungsskalen (1 nicht aussagekräftig -5 exzellent).

Ergebnisse Im Vergleich zu den anderen Untersuchungen hatte CEUS die größte Aussagekraft bei sehr guter Bildqualität und die Fragestellungen konnten zu $100 \%$ beurteilt werden. ceB-Flow, FKDS und B-Flow waren im Vergleich zu CEUS schlechter. Die höchste Eindringtiefe war bei CEUS $(8,6 \pm 1,6 \mathrm{~cm})$, gefolgt von ceB-Flow $(7,0 \pm 1,7 \mathrm{~cm})$ und FKDS $(6,8 \pm 1,6 \mathrm{~cm})$. FKDS hat im Vergleich zu B-Flow und CEUS Einschränkungen bei der Darstellung langsamer Blutflussgeschwindigkeiten. B-Flow erlaubt lediglich die Beurteilung von oberflächlichen Organregionen $(5,0 \pm 1,1 \mathrm{~cm})$ und ist für Bildartefakte sehr anfällig. Durch Kontrastmittelgabe konnte die Eindringtiefe des B-Flow signifikant verbessert werden $(5,0 \pm 1,1 \mathrm{~cm}$ vs. $7,0 \pm 1,7 \mathrm{~cm})$.

Zusammenfassung Digitale Flussverfahren eignen sich insbesondere zur Beurteilung von oberflächlichen Veränderungen und können bei vaskulären Problemen der großen Gefäße wesentliche Zusatzinformationen liefern. Nur CEUS allein hat durch die Darstellung der Mikrozirkulation auf kapillarer Ebene die höchste klinische Aussagekraft. Für die vollständige Beurteilung des Blutflusses von Nierentransplantaten ist die Kombination aller Ultraschall-Verfahren erforderlich. 


\section{07.06 Histologisch gesicherte embolische Pleuraläsionen in der kontrastmittelunterstützten Sonografie (CEUS) bei klinischem Verdacht, jedoch fehlendem Nachweis einer zentralen LAE in der computertomografischen Angiografie (CTA) des Thorax- eine Fallserie}

Autoren Trenker $C^{1}$, Neesse $A^{2}$, Görg $C^{3}$

Institut 1 Zentrum für Innere Medizin, Klinik für Hämatologie, Onkologie und Immunologie; 2 Klinik für Gastroenterologie und gastrointestinale Onkologie, Universitätsklinikum Göttingen; 3 Zentrum für Innere Medizin,

Interdisziplinäres Ultraschallzentrum/1,3 Universitätsklinikum Gießen und Marburg, Standort Marburg und Philipps-Universität Marburg DOI 10.1055/s-0038-1670440

Fragestellung Darstellung von Patienten mit klinischem Verdacht einer Lungenarterienembolie (LAE) und histologisch gesicherter peripherer Pleuraläsionen in der B-Bild- und kontrastmittelunterstützten Sonografie (CEUS), bei fehlendem Embolienachweis in der computertomografischen Angiografie (CTA) des Thorax.

Patienten/Methoden Bei klinischem Verdacht einer LAE erfolge zwischen Januar 2017 und Januar 2018 bei $n=6$ Patienten mit B-Bild sonografischem Nachweis peripherer Pleuradefekte der Ausschluss einer LAE mittels CTA. In allen Fällen wurde eine CEUS der Pleuraläsionen durchgeführt, wobei ein fehlendes oder inhomogenes Enhancement verdächtig für eine embolische Genese galt. Alle Defekte wurden histologisch gesichert. Die Datenauswertung erfolgte retrospektiv.

Ergebnisse Nach dem Wellscore hatten $n=4$ Patienten (66,7\%) ein mittleres, $\mathrm{n}=2(33,3 \%)$ ein hohes Risikoprofil für das Vorliegen einer LAE. Im B-Bild stellten sich alle Läsionen mit einer durchschnittlichen Größe von $2,4 \mathrm{~cm}$ dar, wovon $n=5$ echoarm und $n=1$ echokomplex waren. Die Form war keilförmig ( $n=5)$ oder rund $n=1$, die Anzahl solitär $(n=4)$ oder multiple $(n=2)$. In der CEUS zeigten die Läsionen ein fehlendes $(n=3)$ oder ein inhomogenes $(n=3)$ Enhancement mit Arealen fehlender Kontrastmittelanreicherung. Die Histologie zeigte reine Lungeninfarkte $(n=5)$, sowie in einem Fall einen Lungeninfarkt mit zusätzlichem Nachweis von Tumorzellen.

Schlussfolgerung Bei klinischem Verdacht auf eine LAE schließt der fehlende Nachweis einer zentralen LAE im CTA eine embolische Pleuritis nicht aus. Die kontrastmittelunterstüzte Sonografie erweitert das diagnostische Potential der CTA bei V.a. LAE.

\section{07.07 HIREC und B-Flow CEUS - Kontrastverfahren zur Optimierung der intrahepatischen Gefäßdarstellung}

Autoren Weskott $\mathrm{HP}^{1}$

Institut 1 Ultraschall Ambulanz, Klinikum Siloah, Klinikum Region Hannover DOI 10.1055/s-0038-1670441

Einführung Evaluation der klinischen Einsatzbereiche für die CEUS Untersuchung im HIREC und B-Flow Verfahren (BFC).

Material und Methoden Mittels Low MI Coded Harmonic Imaging werden bei hoher frame rate und einer dem B-Mode ähnlichen Ortsauflösung die arteriellen und portalvenösen Lebergefäße in einem sweep in der arteriellen Phase dargestellt. Das B-Flow Verfahren ist ein Subtraktionsverfahren zur Blutflussdarstellung. Im Kontrastmittelmode wird die akustische Sendeleistung auf 8 $10 \%$ reduziert. Stationäre Echos werden so eliminiert, die Gewebeunterdrückung ist vollständig, womit eine Skelettierung der Gefäßanatomie erreicht wird. Gerät: LOGIQ E9, C1 - 9, C1 - 5 (GE Healthcare, Solingen, Deutschland). Dosis: 1,4mL Sonovue Bolus. Es wurden etwa 10 Patienten mit Portalvenenverschlüssen und 50 Patienten mit Leberzirrhosen ausgewertet.

Resultate Beide CEUS Modi eignen sich für die Darstellung der intraheptischen Gefäßanatomie, bei intrahepatischen Shunts, der Gefäßarchitektur von Leberläsionen, des parabiliären Saintsche und Petrensche Plexus nach
Portalvenenthrombose sowie der Darstellung der intrahepatischen Arterien und Kollateralen bei Leberzirrhose. Mittels HIREC und BFC konnten die Kollateralen vom Leberhilus bis weit in die Leber hinein verfolgt werden (s. Abb. 1). Arterielle und extra-intrahepatischen Portalvenenstenosen können mit ihren Kollateralen dargestellt werden. Alle Leberzirrhosen wiesen eine vorzeitige Parenchymkontrastierung bei vorzeitiger Kontrastierung der Lebervenen auf. Ein pathologisches Gefäßbild der Leberarterien trat vor allem bei postentzündlichen Zirrhosen, PSC und Lebertumoren auf. In 5 Patienten fanden sich arterielle Stenosen.

Schlussfolgerung Mit dem HIREC und BFC stehen kontrastverstärkte Verfahren zur Verfügung, die sensitiv und mit hoher Ortsauflösung Gefäßpathologien der Leber darstellen können. Sie tragen zu einem besseren Verständnis der Leberperfusion und seiner Kontrastkinetik bei.

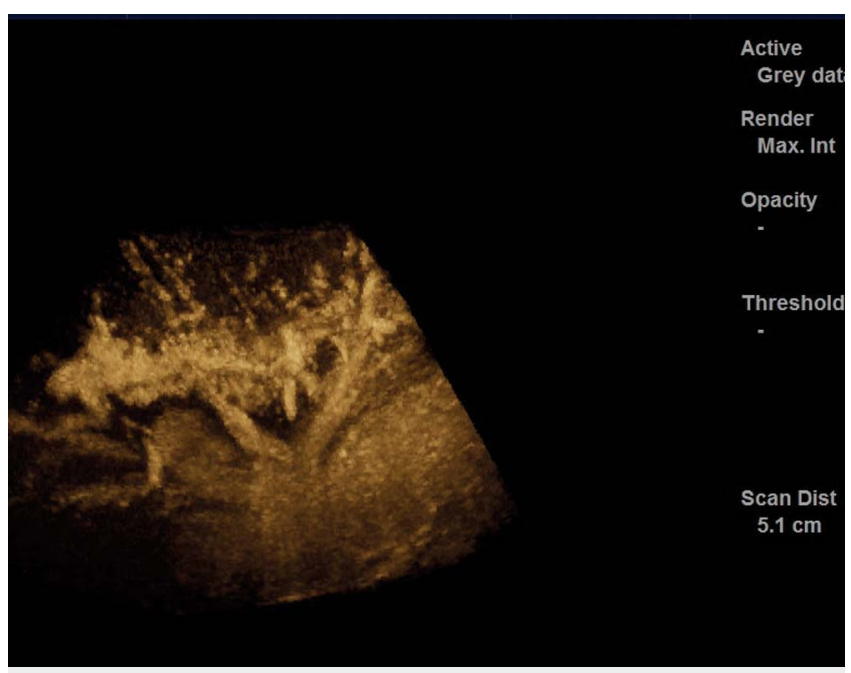

Abb. 1

Wi-Vo 08 Gynäkologie/Geburtshilfe IV

Fr. 16.11. 08:30 - 10:00 Shanghai 3

\section{08.01 Klinischer Verlauf von pränatal diagnostizierten Rhabdomyomen}

Autoren Altmann $\mathrm{J}^{1}$, Kiver $\mathrm{V}^{1}$, Henrich $\mathrm{W}^{1}$, Weichert $\mathrm{A}^{1}$

Institut 1 Klinik für Geburtshilfe, Charité - Universitätsmedizin, Berlin DOI 10.1055/s-0038-1670442

Zielsetzung Es wurden 47 Fälle von Feten mit pränatal vermuteten Rhabdomyomen untersucht und ihr klinischer Verlauf sowie die diesbezügliche Literatur ausgewertet.

Methoden Es wurden klinikeigene Daten von 47 Feten retrospektiv analysiert. Ergebnisse In 5 Fällen wurde, nach genetischer Sicherung einer Tuberösen Sklerose (TSC), die Schwangerschaftsbeendigung gewählt. In 29 Fällen waren postnatale Daten zum Verlauf, maximal bis zum 11. Lebensjahr, verfügbar. In $77 \%$ handelte es sich um multiple Rhabdomyome. Nur 4\% zeigten sich pränatal mit Perikarderguss and 6\% mit einer Extrasystolie, wovon eine mit Digitalis behandelt wurde. Postnatal litten $28 \%$ an Herzrhythmusstörungen wie supra-, oder ventrikulären Tachykardien, WPW-Syndrom oder einem AV-Block. Ein Kind erhielt aufgrund eines 2,8 × 1,6 cm großen Rhabdomyoms, welches den rechtsventrikulären Einflusstrakt verlegte, eine Fontan-Operation mit Glenn-Anastomose. Ein Kind mit einer kardialen Ejektionsfraktion von 15\% starb nach 2 Stunden Reanimation in der Folge einer plötzlich beim Essen aufgetretenen Asystolie. 53\% der Kinder litten an Epilepsie, von Absencen, über West-Syndrom bis hin zu generalisierten tonisch-klonischen Anfällen mit Frequenzen bis zu 40-mal pro Tag. 3 Kinder erhielten eine neurochirurgische Resektion des epileptogenen Fokus. In 32\% wurde die Diagnose 
einer TSC genetisch gesichert. In einem Fall handelt es sich um eine TSC mit einem Lesh-Nyhan-Syndrom. In den anderen Fällen mit deutlichen klinischen Zeichen einer TSC wurde die Diagnose klinisch gestellt. In einem Fall wurde postnatal ein Beckwith-Wiedemann-Syndrom als ursächliche Krankheit identifiziert.

Zusammenfassung Rhabdomyome sind sehr seltene, benigne Tumore. Es besteht eine Assoziation mit einer Tuberösen Sklerose. In der Mehrzahl der Fälle sind Rhabdomyome nicht hämodynamisch relevant und bleiben postnatal konstant in ihrer Größe. Die Lebensqualität der Betroffenen wird vor allem durch die Epilepsie und psychomotorische Retardierung beeinträchtigt.

\section{08.02 Delta MCA-PSV MoM in detecting TAPS}

Authors Fonseca $\mathrm{A}^{1}$, Hecher $\mathrm{K}^{1}$

Institute 1 Geburtshilfe und Pränatalmedizin, Universitätsklinikum HamburgEppendorf

DOI 10.1055/s-0038-1670443

Objectives To evaluate the relationship between inter-twin differences in hemoglobin concentrations at birth and fetal inter-twin differences in middle cerebral artery peak systolic velocities (MCA-PSV) in monochorionic diamniotic twins (MCDA).

Methods This was a retrospective cohort study of MCDA twin pregnancies delivered between January 2012 and January 2018. All pregnancies with measurements of MCA-PSV within seven days of delivery and where neonatal $\mathrm{Hb}$ concentrations were available were included. We investigated the correlation between fetal inter-twin differences of MCA-PSV expressed in multiples of the median (MoM) and neonatal hemoglobin differences.

Results A total of 154 out of $256 \mathrm{MC}$ twin pregnancies fulfilled the inclusion criteria. The fetal inter-twin MCA-PSV MoM differences correlated positively with the neonatal inter-twin hemoglobin differences $(r=0.79$; $P<0.001)$. The 90 th centile for hemoglobin differences was $7.25 \mathrm{~g} / \mathrm{dl}$. There were 15 (9.7\%) cases with a $\mathrm{Hb}$ difference $\geq 7.25 \mathrm{~g} / \mathrm{dl}$ at birth. Receiver-operating characteristic (ROC) curve was constructed and showed a high accuracy of fetal intertwin MCA-PSV MoM differences for the prediction of neonatal inter-twin $\mathrm{Hb}$ difference $\geq 7.25 \mathrm{~g} / \mathrm{dl}$ at birth (area under the curve (AUC), 0.976; 95\% Cl, 0.935 -0.993; $P=0.012$ ). The optimal cut off for MCA-PSV MoM difference was 0.373 with a sensitivity of $93 \%$ and a specificity of $96 \%$. The false positive and false negative rates were $4.3 \%(6 / 139)$ and $6.7 \%(1 / 15)$, respectively. Conclusion Our findings show that fetal inter-twin MCA-PSV MoM difference is a good predictor of neonatal inter-twin hemoglobin difference.

\section{08.03 Pränatale Diagnose struktureller Fehlbildungen bei euploiden Feten in Abhängigkeit vom ETS}

Autoren Eggensberger $\mathrm{T}^{1}$, Ponnath $\mathrm{M}^{2}$, Hatzipanagiotou $\mathrm{M}^{1}$, Huber $\mathrm{D}^{1}$, Inwald $\mathrm{EC}^{1}$, Ortmann $\mathrm{O}^{1}$, Germer $\mathrm{U}^{1}$

Institut 1 Caritas-Krankenhaus St. Josef, Klinik für Frauenheilkunde und Geburtshilfe, Regensburg; 2 Praxis für Frauenheilkunde und

Schwangerenvorsorge, Straubing

DOI 10.1055/s-0038-1670444

Einführung Ziel der Studie ist den Einfluss des Ersttrimesterscreenings (ETS) auf den Detektionszeitraum und das Spektrum struktureller Fehlbildungen bei euploiden Feten zu untersuchen.

Material und Methoden Eingeschlossen in die retrospektive Studie wurden Schwangere mit euploiden Einlingsfeten, die zwischen 2004-13 eine detaillierte Ultraschalluntersuchung erhielten. Erfragt wurden die Durchführung und die Ergebnisse eines vorausgehenden ETS, das durch niedergelassen Gynäkologen ohne Spezialisierung in Pränatalmedizin erfolgt war. Dabei wurden 4 Gruppen gebildet: Gruppe A: Feten ohne ETS; Gruppe B: Feten mit vorausgehendem ETS mit den Subgruppen B1 (unauffälliges ETS) und B2 (auffälliges ETS). Analysiert wurden Detektionszeitraum und die Spektren struktureller Fehlbildungen in den jeweiligen Gruppen.
Resultate 9284 Feten wurden in die Studie eingeschlossen, darunter 513 $(5,5 \%)$ Feten mit strukturellen Fehlbildungen. Diese betrafen am häufigsten das Herzkreislaufsystem ( $n=105,20,4 \%$ ) sowie das Urogenitalsystem ( $n=94$, $18,3 \%$ ). Die Fehlbildungsraten in den Gruppen A, B, B1 und B2 waren 5,4\% $(n=374), 6,0 \%(n=139), 5,5 \%(n=108)$, und 8,7\% $(n=31)$. Die Fehlbildungsraten waren in den Gruppen A und B und B1 ähnlich, wohingegen die Fehlbildungsrate nach auffälligem ETS im Vergleich zu den anderen signifikant höher $(p=0,007)$ war. Betreffend das Spektrum der Fehlbildungen wiesen diese Feten signifikant häufiger eine Fehlbildung des Herz-Kreislauf-Systems auf $(p=0,003)$. Fehlbildungen in Gruppe B wurden signifikant häufiger vor der 18. SSW entdeckt als in Gruppe $A(p=0,016)$. Die Entdeckungsrate der Fehlbildungen vor der 22. SSW in Gruppe B2 lag mit 90,3\% (28/31) über der in Gruppe A und B1 mit 52,1\% (195/374) und 57,4\% (62/108). In Gruppe B wurden $23,7 \%$ (9/38) der kardiovaskulären Fehlbildungen vor der 18. SSW und nur 4,4\% (3/67) in der Gruppe A detektiert.

Schlussfolgerung Auch wenn das ETS auf die Entdeckung von Trisomien ausgerichtet ist, beeinflusst es die pränatale Entdeckung struktureller Fehlbildungen bei euploiden Feten.

\section{08.04 Erhöhte NT bei SSL $\leq 45 \mathrm{~mm}$, Prä- und Postnatales Outcome}

Autoren Kreiselmaier $\mathrm{P}^{1}$, Krapp $\mathrm{M}^{1}$, Ritgen $\mathrm{J}^{2}$, Stressig $\mathrm{R}^{2}$, Papadopoulos $\mathrm{M}^{2}$, Degenhardt j ${ }^{2}$

Institut 1 Zentrum für Pränatalmedizin, amedes experts Hamburg;

2 Praenatal plus, Praxis für Praenatale Medizin und Genetik, Köln

DOI 10.1055/s-0038-1670445

Einführung Die Nackentransparenzmessung bei fetaler Scheitelsteißlänge (SSL) von $45 \mathrm{~mm}$ bis $84 \mathrm{~mm}$ zur Risikoberechnung für das Vorliegen einer Chromosomenstörung ist ein etabliertes Verfahren. Weil in den deutschen Mutterschaftsrichtlinien das 1. Screening zwischen der 9. Und 11. Schwangerschaftswoche (SSW) stattfindet, werden immer wieder Feten mit einer erhöhte Nackentransparenz (NT) und einer SSL $\leq 45 \mathrm{~mm}$ entdeckt. In dieser Situation kann keine Risikoberechnung erfolgen. In dieser retrospektiven Studie haben wir das Outcome bei erhöhter NT und einer SSL zwischen $35 \mathrm{~mm}$ und $45 \mathrm{~mm}$ ausgewertet.

Material und Methoden Im Zeitraum von Februar 2008 bis Februar 2018 wurden in unseren Sprechstunden 150 Einlingsschwangerschaften mit einer SSL zwischen $35 \mathrm{~mm}$ und $45 \mathrm{~mm}$ und einer NT oberhalb der 95. Perzentile für eine SSL von $45 \mathrm{~mm}$ untersucht. Die Messungen der SSL und der NT wurde nach den Vorgaben der FMF London von zertifizierten Untersuchern durchgeführt. Bei allen Feten erfolgte, soweit aufgrund des Gestationsalters möglich, eine frühe Fehlbildungsdiagnostik. Mithilfe der Regelanamnese und der SSLMessung wurde die Schwangerschaftswoche bestimmt. Die Datenerhebung erfolgte über eine retrospektive Abfrage unserer computergestützten Datenbanken. Es wurde das prä- und postnatale Outcome untersucht. Die Informationen dazu wurden entweder aus der computergestützten Datenbank oder durch direkten Anruf bei den Patientinnen ermittelt.

Resultate 150 Einlingsschwangerschaften mit erhöhter NT (2,4-9,1 mm) wurden zwischen 2008 und 2018 untersucht. Die mittlere SSL betrug 40,4 mm (23,2 - $45 \mathrm{~mm})$, bei einem mittleren mütterlichen Alter bei Erstdiagnose von 32 Jahren (17 - 43 Jahre). Bei 51/150 (34\%) Schwangerschaften wurde ein gesunder Einling geboren. Bei 106/150 (71\%) wurde eine invasive Diagnostik durchgeführt, die bei 48 Feten (45\%) einen aufälligen Karyotyp ergab (u.a. 7xTrisomie 21, 23x Trisomie 18, 2x Trisomie 13 und $10 \times 45, \mathrm{XO}$ ). Beim Vergleich der NT von Feten mit auffälligem vs. unauffälligem Karyotyp (5,1 vs. $4,2 \mathrm{~mm}$ ) fand sich eine signifikant höhere NT bei auffälligem Karyotyp $(p=0,004)$. Beim Vergleich der NT von Feten mit und ohne Fehlbildungen (5,0 vs. $4,1 \mathrm{~mm}$ ) zeigte sich ebenfalls ein signifikanter Unterschied $(p<0,01)$.

Schlussfolgerung Bei Feten mit SSL $\leq 45 \mathrm{~mm}$ und erhöhter NT von $\geq 5 \mathrm{~mm}$ besteht eine relativ hohe Wahrscheinlichkeit für ein ungünstiges Outcome im Hinblick auf Chromosomenstörung und/oder Fehlbildung. 


\section{08.05 Kongenital korrigierte Transposition der großen Arterien - Diagnose, Assoziationen und Outcome in 69 pränatal diagnostizierten Fällen}

Autoren Enzensberger $C^{1}$, Kurkevych $A^{2}$, Slodki $M^{3}$, Respondek-Liberska $M^{3}$, Zych-Krekora $\mathrm{K}^{3}$, Stressig $\mathrm{R}^{4}$, Ritgen $\mathrm{J}^{4}$, Rizzo $\mathrm{G}^{5}$, Krapp $\mathrm{M}^{6}$, Mielke $\mathrm{G}^{7}$, Bosselmann $\mathrm{S}^{7}$, Wolter $\mathrm{A}^{1}$, Goette $\mathrm{M}^{1}$, Axt-Fliedner $\mathrm{R}^{1}$; International Prenatal Institut 1 Department of Obstetrics and Gynecology, Division of Prenatal Medicine, University Hospital Gießen and Marburg, Campus Gießen, JustusLiebig University, Gießen; 2 Fetal Cardiology Unit, Ukrainian Children's Cardiac Center, Kyiv; 3 Department of Prenatal Cardiology, Polish Mother's Memorial Hospital Research Institute, Lodz; 4 Prenatalplus.de, Köln; 5 Department Obstetrics and Gynecology, Università di Roma Tor Vergata, Rome; 6 Prenatal Medicine, Amedes Fertility Centre Hamburg, Hamburg, Germany;

7 Praenatalzentrum Stuttgart, Stuttgart

DOI 10.1055/s-0038-1670446

Einführung Die kongenital korrigierte Transposition der großen Arterien (ccTGA) ist ein seltener angeborener Herzfehler. Er ist charakterisiert durch atrioventrikuläre und ventrikuloarterielle Diskordanz. Das Ziel der Studie war die Beschreibung der Anatomie, assoziierter Anomalien und das Outcome pränatal diagnostizierter ccTGA Fälle.

Material und Methoden Retrospektive Beobachtungsstudie über ccTGA Fälle, welche an sieben Studienzentren von 2002 - 2017 pränatal diagnostiziert wurden. Bei allen Feten wurde eine Echokardiografie sowie ein detaillierter Fehlbildungsausschluss durchgeführt. Postnatal wurde die Diagnose entweder durch Autopsie oder durch einen Kinderkardiologen bestätigt. Follow-up Daten wurden durch Patientenunterlagen erhoben.

Resultate Insgesamt wurden 69 Feten pränatal diagnostiziert. Das mittlere Gestationsalter betrug $26 \pm 6$ Wochen. Das mittlere Gestationsalter bei Geburt lag bei $39 \pm 2$ Wochen mit einem mittleren Geburtsgewicht von $3281 \mathrm{~g}$ $\pm 516 \mathrm{~g}$. Eine isolierte ccTGA kam in $9 / 69$ (13\%) Feten vor, zusätzliche intrakardiale Anomalien wurden in 60/69 (87\%) Feten diagnostiziert. Extrakardiale Anomalien wurden in 10/69 (14,5\%) Fällen detektiert. Ein Schwangerschaftsabbruch wurde bei 5/69 (7,2\%) Feten durchgeführt, 56/69 (81,2\%) Feten wurden lebend geboren, eine Schwangerschaft $(1,4 \%)$ ist derzeit noch anhaltend. Elf Feten sind lost for follow up (15,9\%). Bei 4/56 (7,1\%) Neugeborenen kam es zum Versterben während der Neonatalzeit, ein Kind verstarb im Kindesalter (1,8\%). Eine Information über die postnatale Diagnose war in 57 Fällen vorhanden. In 53/57 (93\%) Fällen konnte die pränatale Diagnosis bestätigt werden, in 4 (7\%) der Fälle wich diese von der pränatalen Diagnose ab. Schlussfolgerung In den meisten Fällen ist die ccTGA keine isolierte Pathologie. Obwohl extrakardiale Anomalien selten sind, treten intracardiale Anomalien häufig auf. Pränatal kann die Diagnose mit hoher Sicherheit im zweiten Trimenon gestellt werden. Für die elterliche Beratung ist wichtig, dass das neonatale Outcome der betroffenen Kinder sehr gut ist.

\section{08.06 Inanspruchnahme von NT-Screening, NIP-Test und invasiver Diagnostik im Risikokollektiv in den Jahren 2015 bis 2017}

Autoren Hatzipanagiotou $\mathrm{M}^{1}$, Eggensberger $\mathrm{T}^{1}$, Huber $\mathrm{D}^{1}$, Inwald $\mathrm{E}^{1}$, Ortmann $\mathrm{O}^{1}$, Germer $\mathrm{U}^{1}$

Institut 1 Universitätsfrauenklinik Regensburg

DOI 10.1055/s-0038-1670447

Ziel Das Nackentransparenzscreening (NTS) und der Nicht-invasive Pränataltest (NIPT) sind flächendeckend verfügbare Methoden zur Identifikation von Feten mit einem erhöhten Risiko für Trisomien. Jedoch ist unbekannt in welchem Umfang die o.g. Tests von Schwangeren beansprucht werden und ob sich dieser den letzten Jahren verändert hat. Deshalb wurde die Häufigkeit des NT-Screenings und des NIP-Tests im Risikokollektiv in den Jahren 2015 bis 2017 verglichen.

Methode Befragt und untersucht wurden Einlingsschwangere, die von 2015 bis 2017 in der $19+0$ bis zur 22+0 SSW zur detaillierten Ultraschallun- tersuchung aufgrund diverser Risiken zugewiesen wurden. Die vorherige Inanspruchnahme des NT-Screenings, NIP-Tests bzw. der invasiven Diagnostik wurde in Abhängigkeit vom Untersuchungszeitpunkt erfasst.

Ergebnisse 4829 Schwangere wurden in die Studie eingeschlossen. Dabei wurden in den Jahren 2015, 2016 und 2017 jeweils 1609 (33,3\%), 1543 (32,6\%) und 1677 (34,7\%) Schwangere untersucht. Die Häufigkeiten der Inanspruchnahme der jeweiligen Screening-Methoden sind der folgenden Tabelle zu entnehmen.

\begin{tabular}{|l|l|l|l|l|l|}
\hline Jahr & $\mathbf{n}$ & ETS & NIPT & Beides & Inv. Diagnostik \\
\hline 2015 & $1609(33,3 \%)$ & $563(35,0 \%)$ & $50(3,1 \%)$ & $2(0,1 \%)$ & $110(6,8 \%)$ \\
\hline 2016 & $1543(32,6 \%)$ & $559(36,2 \%)$ & $101(6,5 \%)$ & $10(0,6 \%)$ & $103(6,7 \%)$ \\
\hline 2017 & $1677(34,7 \%)$ & $582(34,7 \%)$ & $139(8,3 \%)$ & $26(1,6 \%)$ & $120(7,2 \%)$ \\
\hline & & $p=0,635$ & $p<0,001$ & & \\
\hline
\end{tabular}

Schlussfolgerung Im Risikokollektiv ließen im Jahr 2015 38,1\% und im Jahr 2017 bereits 43,0\% einen Screeningtest für Trisomien durchführen. Dabei bleibt der Anteil der Schwangeren, die ein NT-Screening oder ETS durchführen lassen konstant bei durchschnittlich 35,3\%. Risikoschwangere entscheiden sich immer noch viermal häufiger für das NTS als für NIPT, jedoch stieg der Anteil der Schwangeren, die einen NIP-Test durchführen ließen, von 3,1\% im Jahr 2015 auf 8,3\% im Jahr 2017 ( $p<0,001$ ). Nur durchschnittlich 6,9\% der Schwangeren des Risikokollektivs lassen vor der Organdiagnostik im zweiten Trimenon eine invasive Diagnostik durchführen, dies bleibt über die Jahre unverändert.

\section{08.07 Zervixlänge vor Fetoskopie bei feto-fetalem Transfusionssyndrom und perinatales Outcome}

Autoren Ortiz $\mathrm{JU}^{1,2}$, Eixarch $\mathrm{E}^{2}$, Micheletti $\mathrm{T}^{2}$, Lobmaier $\mathrm{SM}^{1,2}$, Bennasar $\mathrm{M}^{2}$, Martinez JM², Gratacós $\mathrm{E}^{2}$

Institut 1 Sektion Geburtshilfe und Perinatalmedizin, Technische Universität München; 2 BCNatal - Barcelona Center for Maternal-Fetal and Neonatal

Medicine (Hospital Clínic and Hospital Sant Joan de Deu), IDIBAPS, University of Barcelona, and Centre for Biomedical Research on Rare Diseases (CIBER-ER), Barcelona

DOI 10.1055/s-0038-1670448

Einführung Das Hauptziel dieser Studie war die Auswertung der Assoziation zwischen verschiedenen Zervixlängen-Cut-offs vor einer Fetoskopie bei fetofetalem Transfusionssyndrom (FFTS) und dem perinatalen Outcome. Als sekundäres Ziel wurde die Auswirkung einer Cerclage auf das perinatale Outcome analysiert.

Material und Methoden Eine retrospektive Studie wurde durchgeführt. Monochorial-diamniote Zwillingsschwangerschaften mit FFTS und Zervixmessung innerhalb von $24 \mathrm{~h}$ vor der Fetoskopie wurden eingeschlossen. Die Patientinnen wurden nach der präoperativen Zervixlänge in drei Gruppen eingeteilt ( $\leq 15 \mathrm{~mm}, 16-25 \mathrm{~mm}$ und $>25 \mathrm{~mm}$ ). Das perinatale Outcome wurde dokumentiert und verglichen. Eine Cerclage wurde nur in der Gruppe von Patientinnen mit Zervixlänge $\leq 15 \mathrm{~mm}$ in Erwägung gezogen.

Resultate 330 Patientinnen erfüllten die Kriterien und wurden nach der Zervixlänge wie folgt eingeteilt: $\leq 15 \mathrm{~mm}(\mathrm{n}=30), 16-25 \mathrm{~mm}(\mathrm{n}=29)$ und $>25 \mathrm{~mm}(\mathrm{n}=271)$. Die mediane Zeitspanne zwischen Fetoskopie und Geburt war jeweils 4,6, 11,6 und 13 Wochen $(p<0,001)$. Das mediane Gestationsalter bei der Geburt war 29,3, 34,6 und 34,0 Wochen ( $p<0,001)$. Der Anteil an Geburten $<32+0$ SSW war 79\%, 20\% und 31\% ( $<<0,001)$. Die neonatale Überlebensrate von mindestens einem Zwilling war 68\%, 88\% und 93\% $(p=0,004)$. Es gab keinen Unterschied in Bezug auf Abort und vorzeitigen Blasensprung <32+0 SSW. In der Gruppe mit Zervixlänge $\leq 15 \mathrm{~mm}(\mathrm{n}=30$ ) erhalten 9 Patientinnen eine Cerclage und 21 ein konservatives Management. Das perinatale Outcome war zwischen diesen Subgruppen ähnlich.

Schlussfolgerung Eine extrem verkürzte Zervix $(\leq 15 \mathrm{~mm})$ vor einer Fetoskopie bei FFTS verschlechtert das perinatale Outcome. Allerdings ist eine Fetalchirurgie selbst in dieser Gruppe von Patientinnen vertretbar, da in ca. 2/3 der Fälle mindestens ein Zwilling überlebt. In unserem Kollektiv verbesserte eine Cerclage das Outcome nicht. 
Wi-Vo 09 Pädiatrie/Urologie/Nephrologie

Fr. 16.11. 08:30 - 10:00 Lima

\section{09.01 Resistance Index (RI) in Colour Coded Duplex Sonography (CCDS) - Easy Use and Reliable Results in the Determination of Activity in Infantile Hemangiomas}

\author{
Authors Urban $\mathrm{P}^{1}$ \\ Institute 1 Zentrum Lasermedizin, Evangelische Elisabeth Klinik Berlin \\ DOI 10.1055/s-0038-1670449
}

Purpose Propranolol and laser treatment are helpful tools to solve problems in infantile hemangiomas. The challenge however is to estimate activity i.e. to select those hemangiomas that require treatment. As hemangiomas run through defined stages of development we were looking for an objective criterion to discriminate active from regressive tumours.

Methods 350 consecutive hemangiomas ( 243 female, 107 male; age 1 to 95 months; localization capillitium to foot) were classified corresponding to a clinical and CCDS stage from I to $\mathrm{V}$, we had described in a survey some years ago. In each of these hemangiomas we identified the RI as mean value of 3 measurements in different arteries. Then we related our measurements with the corresponding stages. Finally we performed an ANOVA (analysis of variance) procedure to prove significance.

Results We identified 27 hemangiomas in stage II, 156 in stage III, 155 in stage IV and 12 in stage V. The mean of all RI in stage II was 0,6714 (variance 0,0021 ), in stage III was 0,6047 (variance 0,0025 ), in stage IV was 0,5104 (variance 0,0025 ) and in stage $V$ was 0,4656 (variance 0,0053 ). ANOVA showed that there was highly significant difference ( $p$ value $3,89793 \mathrm{E}-61$ ) of RI between the stages; this could be ensured by the additional post hoc test according to Bonferroni.

Conclusion During the natural development of infantile hemangiomas the flow conditions are changing. This can be measured easily by CCDS and expressed as RI. During the physiological ageing progress in infantile hemangiomas the RI constantly decreases, and shows characteristic figures for each stage of development. As these figures differ significantly it is possible to stage hemangiomas by an objective measurement. This could be important for selecting those hemangiomas with need for treatment and vice versa helping to avoid needless therapy.

\section{09.02 Lungensonografie bei kleinen Frühgeborenen}

Autoren Schwarz $\mathrm{S}^{1}$, Brevis $\mathrm{F}^{1}$, Feldkamp $\mathrm{A}^{1}$

Institut 1 Klinik für Kinder- und Jugendmedizin, Sana Kliniken Duisburg DOI 10.1055/s-0038-1670450

Einführung Das Atemnotsyndrom und die Bronchopulmonale Dysplasie gehören zu den häufigsten Erkrankungen kleiner Frühgeborener. Welche sonografischen Auffälligkeiten bei dieser Patientengruppe festzustellen sind, haben wir bei 23 Patienten analysiert.

Material und Methoden Von November 2017 bis April 2018 wurden bei 23 Frühgeborenen (23+0 bis 29+4 SSW, GG $290 \mathrm{~g}$ bis $1240 \mathrm{~g}$ ) zwischen dem 1. Lebenstag und der 36+0 SSW Ultraschalluntersuchungen der Lunge durchgeführt. Verwendet wurde dabei das Ultraschallgerät GE LOGIQ S8 mit hochauflösenden Linearschallköpfen (ML6-15/L8 - 18). Untersucht wurde senkrecht zum Thorax, möglichst in allen Quadranten.

Resultate Auffälligkeiten der Pleuralinie (verdickt, grobkörnig, irregulär, fehlend) sowie ein interstitielles Syndrom unterschiedlicher Ausprägung fanden sich bei den meisten Untersuchungen. Bei keinem Patienten ohne Sauerstoffbedarf sahen wir allerdings Konsolidierungen oder eine weiße Lunge bds. Alle Frühgeborenen mit einem Sauerstoffbedarf $\geq 30 \%$ zeigten entweder Konsolidierungen oder eine weiße Lunge bds. Dabei fanden sich Konsolidierungen bei $97,5 \%$ und eine weiße Lunge bds. bei 37,5\%. Bei 35\% der Untersuchungen dieser Patientengruppe sahen wir sowohl eine weiße Lunge bds. als auch Konsolidierungen.

Schlussfolgerung In unserem Patientenkollektiv sahen wir bei allen Frühgeborenen mit einem Sauerstoffbedarf $\geq 30 \%$ Konsolidierungen oder eine weiße Lunge bds. Die weiße Lunge gilt als sonografisches Zeichen für ein interstitielles Lungenödem und Hauptmerkmal des Atemnotsyndroms. Konsolidierungen sind das sonografische Korrelat für Belüftungsstörungen wie Atelektasen, welche ein Kennzeichen einer sich entwickelnden Bronchopulmonalen Dysplasie darstellen. Beide Befunde fanden sich sowohl in den ersten Lebenstagen als auch in den folgenden Lebenswochen bei Frühgeborenen mit anhaltend hohem Sauerstoffbedarf. Bestand im Verlauf kein Sauerstoffbedarf mehr, konnten diese Befunde nicht mehr erhoben werden. Das Ausmaß der sonografischen Auffälligkeiten korrelierte dabei gut mit dem Grad der respiratorischen Insuffizienz. Die Lungensonografie bietet somit die Möglichkeit bettseitig und ohne Strahlenbelastung die beiden Komponenten interstitielles Lungenödem und Belüftungsstörungen zu diagnostizieren, ihre Ausprägung zu bewerten und entsprechende Therapieversuche einzuleiten. Dabei sollte immer eine möglichst vollständige Untersuchung der Lunge von allen Seiten angestrebt werden.

\section{09.03 Beziehungen zwischen der sonografisch ermittelten Parenchymfläche und der nuklearmedizinisch bestimmten Funktion durch Mag3-Szintigrafie}

Autoren Weitzel $D^{1}$, Maier $R^{2}$, Müller $C^{2}$, Landvogt $C^{2}$, Hohenfellner $K^{3}$ Institut 1 1)Pädiatrie, Deutsche Klinik für Diagnostik Wiesbaden; 2 Nuklearmedizinische Praxis in der Deutschen Klinik für Diagnostik;

3 Kindernephrologie, Rosenheim

DOI 10.1055/s-0038-1670451

Einleitung Mitentscheidend für die Indikation zu kinderurologischen Operation sind Funktions-Untersuchungen der Nieren. Ausgehend von der Hypothese, dass normales Wachstum mit einer normalen Funktion einhergeht, prüften wir, inwieweit die sonografisch bestimmbare Parenchymfläche Rückschlüsse auf die Funktion ermöglicht.

Methode Untersucht wurden 201 Patienten, darunter 122 Erstuntersuchungen und 79 Verlaufsuntersuchungen. In einem Zeitraum von $+/-3$ Monaten wurden diese Patienten sonografisch und nuklearmedizinisch untersucht. Wir prüften, a) in wieweit der Funktions-Anteil je Niere innerhalb oder außerhalb des Konfidenzbereichs der Parenchymfläche lag und b) inwieweit der Funktionsanteil mit dem Parenchymflächen-Anteil korreliert.

Ergebnisse Bei 71 Nieren mit einem Funktions-Anteil von kleiner $40 \%$ lagen bei 40 Nieren (56\%) die Parenchymflächen unterhalb des 95\%-Konfidenzbereiches. Bei 73 Nieren mit einem Funktionsanteil oberhalb von $60 \%$ war hingegen nur in 19\% (14 von 73) die Parenchymfläche oberhalb des 95\%-Konfidenzbereiches auf. Die Parenchymflächen-Anteile korrelieren eng mit den Funktions-Anteilen $(R=0,794)$. Abb. 1. Regression des Linken Parenchymflächen-Anteils mit dem linken Funktions-Anteil der Niere. Bei Vorliegen von Harnwegserweiterungen war der Korrelationskoeffizient zwischen Parenchymflächen-Anteil und Funktions-Anteil $(R=0,86)$ deutlich höher als bei der Indikation Harnwegsinfektion $(R=0,789)$. Die grafische Darstellung der Veränderung der Parenchymflächen im Verlauf unterstützt die Hypothese, dass normales Parenchym-Flächenwachstum mit einer normalen Funktion einhergeht und ein unterhalb des Konfidenzbereiches liegendes Wachstum mit einer eingeschränkten Funktion verbunden ist.

Schlussfolgerungen Die Bestimmungen der Nierenfläche und des Sinus renalis ermöglichen eine sichere Quantifizierung des Nierenparenchyms. Sie spiegeln das Wachstum des Nierenparenchyms wider und erlauben dadurch Rückschüsse auf die Funktion. Messungen der Nierenflächen sollten daher integraler Bestandteil der Verlaufskontrolle bei kinderurologischer Patienten sein. 


\section{09.04 Kontrastverstärkter Ultraschall (Contrast Enhanced UltraSound = CEUS) bei intravenöser Anwendung in der Pädiatrie - wertvolle und sichere Ergänzung der pädiatrischen Bildgebung}

Autoren Lorenz $N^{1}$, Müller $G^{1}$, Haufe $T^{2}$, Tietze $U^{2}$, Steffgen $L^{3}$, Heubner $G^{1}$ Institut 1 Perinatalzentrum Dresden; 2 Kinderradiologie; 3 TrainingsZentrum Ultraschall-Diagnostik, Wachholder, Mainleus/1 - 2 Städtisches Klinikum Dresden

DOI 10.1055/s-0038-1670452

Einleitung In der Europäischen Union hat die European Medicines Agency Ultraschallkontrastmittel zur intravenösen Anwendung kontrastverstärkter UItraschall-Untersuchungen (CEUS) im Kindes- und Jugendalter bisher nicht zugelassen. Das Positionspapier der EFSUMB (European Federation of Societies for Ultrasound in Medicine and Biology) von 2016 gibt jedoch den in der pädiatrischen Ultraschalldiagnostik Tätigen eine wertvolle Hilfestellung, CEUS als in der Erwachsenenmedizin etablierte Methode „off-label“ zur Diagnosestellung in der Pädiatrie einzusetzen.

Methode Darstellung der Ergebnisse aller an der Kinderklinik Dresden-Neustadt seit 2016 durchgeführten intravenösen CEUS-Untersuchungen. Indikationsstellung und Durchführung entsprechend dem oben aufgeführten EFSUMB-Positionspapier. In jedem einzelnen Fall erfolgte eine eingehende individuelle Aufklärung der Eltern und ggf. Jugendlichen mit Darstellung von Indikation, des off-label-Charakters der Untersuchung, den Alternativen zu CEUS und dem Nebenwirkungsprofil des verwendeten Ultraschallkontrastmittels Sonovue ${ }^{\circledR}$. Zudem erhielten alle Eltern ein selbstentwickeltes, standardisiertes Aufklärungsformular und erklärten ihr schriftliches Einverständnis. Alle Patienten wurden mit liegendem venösen Zugang eine Stunde nachbeobachtet und, wenn aus Altersgründen möglich, nach unerwünschten Wirkungen befragt.

Ergebnisse Seit der Einführung der Untersuchung 2016 wurden 49 Untersuchungen bei 35 Kindern und Jugendlichen im Alter von 1 Tag bis 17 Jahren mit intravenöser Gabe von Sonovue ${ }^{\circledR}$ durchgeführt (Anwendungen/Anzahl: Leber 13, Milz 5, Nieren 13, Hoden 8, Darm 2, Lymphknoten 5, Lunge, Trauma je 1). Lediglich ein Elternteil einer Jugendlichen lehnte die CEUS-Untersuchung ab und wählte stattdessen ein MRT (intraabdomineller Abszess). Bei keinem der Patienten wurden unerwünschte Wirkungen beobachtet.

Diskussion CEUS war in unserem Setting eine sichere und von Eltern und ggf. Patienten akzeptierte Methode. Kontrastmittelgestützte Ultraschalluntersuchungen sind im Kindes- und Jugendalter rasch und ggf. bettseitig durchführbar und erlauben eine valide bildgebende Diagnostik bei Raumforderungen der Leber, bei Ausschluss/Bestätigung u. a. einer Hodentorsion, bzw. einer tubulointerstitiellen Nephritis oder eines Nierenabszesses. Neben der Zeitersparnis konnten kostenintensive MRT vermieden werden. Die insgesamt seltene Anwendung von CEUS in der Pädiatrie erfordert den intensiven kollegialen Austausch und vertiefende wissenschaftliche Diskussion.

\section{09.05 Verbesserung des Blutflusses in Dialysefiltern: Was können moderne Ultraschallverfahren leisten?}

Autoren Putz $\mathrm{FJ}^{1}$, Banas $\mathrm{B}^{1}$, Jung $\mathrm{EM}^{2}$

Institut 1 Abteilung für Nephrologie; 2 Interdisziplinäres Ultraschallzentrum, Institut für Röntgendiagnostik/1 - 2 Universitätsklinikum Regensburg DOI 10.1055/s-0038-1670453

Hintergrund Die Qualität der Hämodialyse ist von vielen verschiedenen Faktoren abhängig, aber wird zu großem Maß von der Güte des Dialysefilters bestimmt. Moderne Dialysefilter bestehen aus einem umspülten Kapillarbündel, welches niedermolekulare Stoffe aus dem Blut durch Diffusion und Filtration entfernt. Neben der Verbesserung der Membraneigenschaften stellt die Optimierung des Blut- und Dialysatflusses einen wesentlichen Parameter zur Verbesserung der Dialyseeffektivität dar. Ziel dieser Arbeit ist es, die Funktion von Dialysefiltern mit den Methoden moderner Ultraschalldiagnostik zu überprüfen.

Material \& Methoden In dieser Machbarkeitsstudie wurden 10 Dialysefilter, 5 fabrikneue Filter und 5 gebrauchte Filter nach Dialysebehandlung mit modernen Ultraschallverfahren untersucht. Die Dialysefilter waren in den Kreislauf einer Dialysemaschine integriert und wurden mit isotonischer Kochsalz-Lösung bei üblichen Flussparametern perfundiert. Die Untersuchung erfolgte online mit einem high-end Ultraschallgerät (LOGIQ E9, GE, USA). Nach initialer Optimierung der Bildeinstellungen, wurden die Dialysatoren im B-Bild, Farbdoppler, digitalen Doppler-Verfahren und mit Kontrastmittelultraschall dynamisch untersucht. Als Ultraschallkontrastmittel wurde eine verdünnte Lösung von Schwefelhexafluorid-Mikrobläschen (SonoVue ${ }^{\circledR}$, Bracco, Italien) verwendet. Die Auswertung des Bildmaterials erfolgte mithilfe der integrierten Gerätesoftware.

Ergebnisse Im konventionellen B-Bild, dem Farbdoppler und modernen digitalen Dopplermethoden konnte kein Flussprofil des Dialysefilters dargestellt werden und eine Unterscheidung zwischen fabrikneuen und gebrauchten Dialysatoren gelang nicht. Lediglich die dynamische Untersuchung durch Kontrastmittelultraschall konnte das charakteristische Flussprofil bei den Dialysatoren beschreiben. Hier zeigten sich signifikant unterschiedliche Flussmuster zwischen beiden Gruppen. Das Kontrastmittel verblieb streng intrakapillär, es konnte kein Übertritt in die Dialysatflüssigkeit beobachtet werden, so dass es sich um ein gutes Modell zur Beschreibung des regionalen Blutflusses handelt.

Zusammenfassung Durch moderne Ultraschallmethoden, insbesondere durch die Möglichkeiten des Kontrastmittelultraschalls ist es möglich die regionale Flussverteilung im Dialysefilter online zu beurteilen. Diese neue Methode kann Informationen zur Verbesserung in der Architektur von Dialysefiltern dienen, sowie Strategien zur Antikoagulation überprüfen.

\section{09.06 Imaging of kidney cysts and cystic kidney diseases in children. Consensus paper by an ad hoc committee}

Authors Gimpel $\mathrm{C}^{1}$, Avni $\mathrm{EF}^{2}$, Breysem $\mathrm{L}^{3}$, Burgmaier $\mathrm{K}^{4}$, Caroli $\mathrm{A}^{5}$, Cetiner $\mathrm{M}^{6}$, Haffner $\mathrm{D}^{7}$, Hartung $E A^{8}$, Franke $D^{7}$, König J $\mathrm{J}^{9}$, Liebau $M C^{10}$, Mekahli $D^{11}$, Ong $A C M^{12}$, Pape $L^{7}$, Titieni $A^{9}$, Torra $R^{13}$, Winyard PJD $D^{14}$ Institute 1 Department of General Pediatrics, Adolescent Medicine and Neonatology, Center for Pediatrics, Medical Center - University of Freiburg; 2 Department of Pediatric Radiology, Jeanne de Flandre Mother and Child Hospital, University of Lille; 3 Department of Pediatric Radiology; 4 Department of Pediatrics; 5 Department of Bioengineering, IRCCS Mario Negri Institute for Pharmacological Research, Bergamo; 6 Department of Pediatrics II, University Hospital Essen; 7 Department of Pediatric Kidney, Liver and Metabolic Diseases, Hannover Medical School; 8 Division of Nephrology, Children's Hospital of Philadelphia, University of Pennsylvania, Philadelphia; 9 Department of General Pediatrics, University Children's Hospital, Münster; 10 Department of Pediatrics and Center for Molecular Medicine; 11 Department of Pediatric Nephrology; 12 Academic Nephrology Unit, Department of Infection Immunity \& Cardiovascular Disease, University of Sheffield; 13 Department of Nephrology, Fundacio Puigvert, Autonomous University Barcelona; 14 University College London Great Ormond Street Institute of Child Health; 15 Division of Pediatric Nephrology, Center for Pediatrics and Adolescent Medicine, Heidelberg University Hospital/3,11 University Hospital of Leuven/4,10 University Hospital of Cologne

DOI 10.1055/s-0038-1670454

Introduction Cystic nephropathies in childhood can present with focal pathology (simple and complex kidney cysts), affecting a whole kidney (e.g. multicystic dysplastic kidney, cystic dysplasia), or both kidneys (e.g. autosomal recessive and dominant polycystic kidney disease). In contrast to adults genetic diseases (e. g. HNF1b disease, tuberous sclerosis complex, Bardet-Biedl 
syndrome and other ciliopathies) are much more frequent than acquired cystic kidney disease (mainly after organ transplantation).

Purpose The consensus statement provides clinical guidance on imaging kidney cysts children.

Methods An ad hoc committee with international experts from pediatric nephrology, pediatric radiology, pediatric ultrasound and adult nephrology prepared systematic literature reviews and formulated recommendations at a consensus meeting. The final statement was endorsed by the European Society of Paediatric Radiology (ESPR), the European Federation of Societies for Ultrasound in Medicine and Biology (EFSUMB), the European Society of Pediatric Nephrology (ESPN) and the European Reference Network for Rare Kidney diseases (ERKNet).

Recommendations Ultrasound is the method of choice for diagnosing most pediatric cystic nephropathies, with selected indications for MRI and contrastenhanced ultrasound. CT should be avoided. Diagnostic features are pathognomonic for some diseases, but especially ciliopathies show unspecific findings. Transabdominal ultrasound of the genital tract for associated anomalies is warranted in suspicion of HNF1b disease. In ARPKD and other ciliopathies liver ultrasound should be performed at diagnosis and for repeated screening of portal hypertension. Ultrasound is usually sufficient for follow-up kidney imaging, but MRI can be valuable for clinical trials in ADPKD or older children with TSC and both cysts and angiomyolipomas.

Wi-Vo 10 Abdomen

Fr. 16.11. 10:30 - 12:00 Shanghai 2

\section{10.01 Einfluss der Nahrungsaufnahme auf die Lebersteifigkeit mittels 2D-SWE an 100 gesunden Probanden}

Autoren Petzold $G^{1}$, Porsche $M^{1}$, Ellenrieder $V^{1}$, Kunsch $S^{1}$, Neesse $A^{1}$ Institut 1 Klinik für Gastroenterologie und gastrointestinale Onkologie, Universitätsmedizin Göttingen DOI 10.1055/s-0038-1670455

Einleitung Die Lebersteifigkeit (LS) zeigt eine gute Korrelation dem Fibrosegrad der Leber. Bei der Interpretation der Messwerte sind jedoch verschiedene Einflussfaktoren zu beachten. Einer davon ist die Nahrungsaufnahme. Das Ziel der Studie ist es, den Einfluss der Nahrungsaufnahme auf die LS, gemessen mittels 2D-Scherwellen Elastografie (Logiq E9, GE), bei gesunden Probanden zu untersuchen und die Messwerte mit der Pfortaderflussgeschwindigkeit (PFG) und klinischen Parametern zu korrelieren.

Material und Methoden 100 gesunden Probanden wurden prospektiv eingeschlossen. Die Probanden waren mindestens $3 \mathrm{~h}$ vor der Messung von $\mathrm{LS}$ und PFG nüchtern. Danach nahmen die Probanden eine standardisierte Flüssigmahlzeit $(400 \mathrm{ml}$, Energiegehalt $800 \mathrm{kcal}$ ) unter Aufsicht ein. Innerhalb von 30 bis 40 Minuten nach der Nahrungsaufnahme erfolgte eine erneute Messung von LS und PFG. Unterschiede der LS und PFG präprandial und postprandial sowie der Einfluss klinischer Parameter wurden analysiert. Alle Messungen wurden von demselben Untersucher durchgeführt.

Resultate Die Erfolgsrate der 200 LS-Messungen lag bei 100\%. Die Nahrungsaufnahme führte zu signifikant höheren LS-Werten im Vergleich zu den präprandialen Messungen $(5,74( \pm 0,94) \mathrm{kPa}$ vs. 4,80 $( \pm 0,94) \mathrm{kPa}, \mathrm{p}<0,001)$. Die Dauer der Nüchternperiode ( $3-5$ Stunden oder $>6 \mathrm{~h}$ ) hatte keinen signifikanten Einfluss auf die präprandialen LS-Werte. Diese waren bei Männern $(n=48$; $5,09( \pm 0,95) \mathrm{kPa})$ signifikant höher als bei Frauen $(n=52 ; 4,53( \pm 0,85) \mathrm{kPa}$; $p=0,002)$. Des Weiteren zeigte die präprandiale LS eine negative Korrelation mit dem absoluten Anstieg der LS nach Nahrungsaufnahme $(r=-0,392)$. Die präprandiale PFG $(22,3( \pm 3,4) \mathrm{cm} / \mathrm{s})$ war signifikant niedriger als postprandial $(36,0( \pm 5,3) \mathrm{cm} / \mathrm{s}, \mathrm{p}<0,001)$. Jedoch zeigte sich keine Korrelation zwischen dem Anstieg der LS und dem Anstieg der PFG postprandial $(r=0,09)$.

Schlussfolgerung Die Nahrungsaufnahme hat einen signifikanten Einfluss auf die LS. Dadurch besteht ein Risiko von bis zu $11 \%$, gesunde Probanden als leberkrank mit signifikanter Fibrose $(F \geq 2)$ falsch zu klassifizieren. Wir empfehlen, die Messung der LS unter Fastenbedingungen (mindestens 3 Stunden) vorzunehmen.

\section{10.02 Nicht-invasives Fettleberscreening in einer kardiovaskulären Risikokohorte}

Autoren Beer $\mathrm{S}^{1,2}$, Babel $J^{1}$, Neef $\mathrm{M}^{3}$, Keim $\mathrm{V}^{1}$, Wiegand $\mathrm{J}^{1}$, Karlas $\mathrm{T}^{1}$ Institut 1 Klinik und Poliklinik für Gastroenterologie und Rheumatologie; 2 Integriertes Forschungs- und Behandlungszentrum für

Adipositaserkrankungen Leipzig; 3 Klinik und Poliklinik für Kardiologie/ 1,3

Universitätsklinikum Leipzig

DOI 10.1055/s-0038-1670456

Eine Assoziation von kardiovaskulären Erkrankungen, insbesondere der koronaren Herzerkrankung (KHK), und Fettlebererkrankungen (FLD) ist bekannt. Ob ein Screening auf eine FLD zur Risikobeurteilung bezüglich einer KHK sinnvoll ist oder ob für besondere Risikokohorten ein FLD-Screening durchgeführt werden soll, ist bislang nicht ausreichend definiert. In dieser Studie erfolgte an einer für KHK und Komorbiditäten definierten Kohorte eine erweitertes FLD-Screening, um dessen Stellenwert zu prüfen.

Bei 183 Patienten (M:122, W:61), die für eine Koronarangiografie (KA) vorgesehen waren, wurde prospektiv eine Sonografie, eine Lebersteifigkeitsmessung durch transiente Elastografie (TE) und Leberfettquantifizierung (controlled-attenuation-parameter, CAP), sowie eine umfangreiche Charakterisierung zu Komorbiditäten, Lebensgewohnheiten und Laborparametern durchgeführt. Die Patienten waren im Mittel $66 \pm 9,8$ Jahre alt und hatten einem BMI von $29,0 \pm 4,2 \mathrm{~kg} / \mathrm{m}^{2}$. 97 Patienten wiesen eine interventionspflichtige KHK auf und 80 zeigten eine unauffällige KA. Entgegen der bekannten Assoziation zeigte sich kein signifikanter Unterschied zwischen Patienten mit und ohne KHK bezüglich des CAP ( 271 vs. $269 \mathrm{~dB} / \mathrm{m}$ ) oder TE (5,4 vs. 4,9 kPa; $p=0,089)$, jedoch ein Trend für erhöhte TE bei KHK. Betrachtet man nur Patienten, welche erstmalig eine KA erhalten findet sich ein signifikanter Unterschied der TE zwischen Patienten mit KHK und ohne KHK (5,4 vs. 4,6 kPa; $p=0,015)$. In der gesamten Kohorte ist ein erhöhter Alkoholkonsum $(n=57)$ von mindestens $175 \mathrm{~g} /$ Woche $(25 \mathrm{~g} / \mathrm{d})$ mit einer erhöhten TE gegenüber der Gruppe der absoluten Nicht-Trinker $(n=40)$ assoziiert $(5,7$ vs. 4,4; $p=0,027)$. Der CAP zeigt sich hierbei nur tendenziell erhöht ( 279 vs. $263 \mathrm{~dB} / \mathrm{m} ; \mathrm{p}=0,16$ ). Betrachtet man alle Diabetiker ( $n=53,29 \%$ ), so zeigen sich signifikante Unterschiede der TE (6,2 vs. 4,7 kPa; $p=0,013)$, sowie des CAP (281 vs. $265 \mathrm{~dB}$ / $m ; p=0,027)$ gegenüber den Nicht-Diabetikern. Dies ist nach Ausschluss von erhöhtem Alkoholkonsum (>210 bzw. $140 \mathrm{~g} /$ Woche; $\mathrm{n}=61$ ) allerdings nicht mehr signifikant (TE 5,2 vs. 4,8 kPa; $\mathrm{p}=0,63$; CAP 277 vs. $271 \mathrm{~dB} / \mathrm{m} ; \mathrm{p}=0,24$ ). Patienten mit KHK-Verdacht haben eine hohe Prävelanz der FLD definiert durch erhöhte CAP. Das Ausmaß der nicht-invasiv ermittelten Leberverfettung ist jedoch nicht mit der KHK assoziiert. Die TE korreliert bei Erstdiagnostik mit dem KHK-Risiko. Neben Diabetes ist vor allem ein regelmäßiger Alkoholkonsum mit erhöhter TE assoziiert. Diese Risikopatienten können von einer erweiterten FLD Diagnostik profitieren.

\section{10.03 Wertigkeit der Sonografie in der Diagnostik der Leistenhernie - eine retrospektive Follow-up-Studie}

Autoren Maisenbacher $\mathrm{T}^{1}$, Kratzer $\mathrm{W}^{1}$, Formentini $\mathrm{A}^{2}$, Schmidberger $\mathrm{J}^{1}$, Hänle $M M^{1}$, Henne-Bruns $D^{2}$, Gräter $T^{3}$, Hillenbrand $A^{2}$

Institut 1 Klinik für Innere Medizin I; 2 Klinik für Allgemein- und

Viszeralchirurgie; 3 Klinik für diagnostische und interventionelle Radiologie/

1 - 3 Universitätsklinikum Ulm

DOI 10.1055/s-0038-1670457

Einführung Leistenhernien werden primär klinisch diagnostiziert. Eine genaue Bildgebung erscheint notwendig, wenn die klinische Untersuchung kein eindeutiges Ergebnis liefert. Ziel dieser Studie war es, die Wertigkeit der Sonografie in der Diagnostik von Leistenhernien zu bestimmen und deren Einfluss 
in der Entscheidungs-findung für oder gegen eine Operation herauszuarbeiten.

Material und Methode Es handelte sich bei dieser Studie um eine retrospektive Studie, die als Single-Center-Studie von Januar 2012 bis Dezember 2016 durchgeführt wurde. Untersucht wurden 326 Patienten, die zur differentialdiagnostischen Abklärung eine Ultraschalluntersuchung der Leiste erhalten hatten. Neben Operationsbefunden als Goldstandard wurden Angaben aus einem Follow-up und alternative sonografische Diagnosen als Referenz betrachtet, um den Stellenwert der Sonografie bei negativen Ultraschallbefunden möglichst genau einschätzen zu können.

Resultate Die Sonografie war bei 248 Patienten positiv und bei 78 Patienten negativ. 201 Patienten wurden operiert und weitere 40 Patienten konnten durch einen Fragebogen und die alternativen sonografischen Diagnosen validiert werden. Die Korrelation mit diesen Referenzen ergab eine Sensitivität von $97 \%$, eine Spezifität von $77 \%$, einen positiv prädiktiven Wert von $95 \%$ und einen negativ prädiktiven Wert von $87 \%$.

Schlussfolgerungen Die Sonografie stellt eine sehr genaue Methode für die Evaluierung von Leistenhernien dar. Mit einer hohen Sensitivität eignet sie sich bei einem negativen Ultraschallbefund insbesondere gut zum Ausschluss einer Leistenhernie. Eine zusätzlich zur klinischen Untersuchung durchgeführte Sonografie kann daher die korrekte Indikation und Entscheidung für eine chirurgische Intervention vereinfachen.

\section{10.04 Vergleich der Scherwellen-Elastografie- Messung der Leber zwischen Siemens Acuson S3000, GE LOGIQ E9, Philips EPIQ7 and Toshiba Aplio 500 (software version 5.0 and 6.0)}

Autoren Gress VS ${ }^{1}$, Glawion EN ${ }^{1}$, Schmidberger J', Kratzer W' Institut 1 Klinik für Innere Medizin I, Universitätsklinikum Ulm DOI 10.1055/s-0038-1670458

Einführung Vergleich der ARFI-basierten Elastografieverfahren der Sonografie-geräte GE LOGIQ E9, Philips EPIQ 7 und Toshiba Aplio 500 (Softwareversion 5.0 und 6.0) gegen das Referenzgerät Siemens Acuson S3000, an Lebergesunden, unter Berücksichtigung verschiedener Einflussfaktoren.

Material und Methoden Die Studie bestand aus zwei Folgestudien: In der ersten Studie ( $n=205)$ wurden die 2D-SWE Geräte GE und Toshiba 5.0 sowie das pSWE Gerät von Philips gegen das Siemens-Referenzgerät getestet. In einer zweiten Studie $(n=113)$ wurde an einem weiteren Kollektiv Toshiba 6.0 mit Siemens verglichen. Insgesamt erfüllten 318 (M:F=122:196) von 433 Studienteilnehmern die Einschlusskriterien. Die Elastografie-Messungen wurden im rechten Leberlappen in 3,5 und $7 \mathrm{~cm}$ Tiefe durchgeführt. Für Siemens und Philips wurde pro Tiefe je fünf, für GE und Toshiba jeweils eine Messung durchgeführt.

Resultate Die stärkste Korrelation der Scherwellengeschwindigkeiten zwischen Siemens und Vergleichsgeräten konnte für Philips $(r=0,58$, $p<0,0001)$ und $G E(r=0,63, p<0,0001)$ in $5 \mathrm{~cm}$ Messtiefe erfasst werden. Bei Toshiba (Softwareversion 6.0) fand sich die stärkste Korrelation in $3 \mathrm{~cm}$ Messtiefe $(r=0,57, p<0,0001)$. Die Toshiba-Softwareversion 5.0 lieferte keine zufriedenstellenden Ergebnisse. Gerätespezifische Unterschiede der Messwerte zeigen sich für die Einflussfaktoren Geschlecht und BMI $(p<0,05)$. Das Alter der Probanden zeigte keinen Einfluss auf die Messwerte.

Schlussfolgerungen Alle untersuchten Geräte, mit Ausnahme der ToshibaSoftwareversion 5.0, können für die Messtiefen von 3 und $5 \mathrm{~cm}$ uneingeschränkt für vergleichende Ultraschallelastografie-Untersuchungen an Lebergesunden empfohlen werden.

\section{10.05 Bildgebende Nachsorge nach endoskopischer Vollwandresektion von Adenomen am Appendixabgang}

\author{
Autoren Grotz $\mathrm{A}^{1}$, Riecken $\mathrm{B}^{1}$, Caca $\mathrm{K}^{1}$, Klinger $\mathrm{C}^{1}$ \\ Institut 1 Medizinische Klinik I, Klinikum Ludwigsburg \\ DOI 10.1055/s-0038-1670459
}

Einführung Die endoskopische Vollwandresektion („endoscopic full thickness resection“, EFTR) ist eine neue Technik zur minimal-invasiven Behandlung komplizierter Kolonadenome (z.B. Lokalisation im Bereich des Appendixabgangs), welche bisher häufig eine operative Behandlung erforderten. Neben dem Adenom wird meist auch die Appendix partiell oder komplett reseziert (Abb. 1). Die Datenlage zur Sicherheit der Methode und zum Stellenwert des Ultraschalls in der Nachsorge ist bisher unzureichend.

Material und Methoden Von 09/2013 bis 02/2018 wurden am Klinikum Ludwigsburg 33 Patienten mit Adenomen am Appendixabgang mittels EFTR behandelt. Alle Patienten erhielten periinterventionell eine Antibiotikaprophylaxe für 3-5 Tage. Es erfolgte eine retrospektive Auswertung der Krankenakten unter Fokussierung auf Komplikationen und die bildgebende Nachsorge.

Resultate Im Verlauf der Nachbeobachtung (median 6,2 Monate; IQR 3-12 Monate) erhielten 21 Patienten (63,9\%) eine Ultraschalluntersuchung und 13 Patienten $(39,4 \%)$ eine Computertomografie. 7 asymptomatische Patienten erhielten keine bildgebende Nachsorge. Bei 18 Patienten (54,5\%) traten postinterventionell Beschwerden auf. Unterschieden wurden schwerwiegende (Major-) und weniger schwerwiegende (Minor-) Komplikationen. Diese sind in Tabelle 1 zusammengefasst. Zusätzlich zeigten sich in der bildgebenden Nachsorge asymptomatische Befunde wie Pseudopolypen durch die Darmwandduplikatur oder eine Mukozele im verbliebenen Appendixstumpf.

\begin{tabular}{|c|c|c|c|}
\hline & Komplikation & Häufigkeit & Therapie \\
\hline \multirow[t]{4}{*}{ Minor } & Nachblutung & $12 \%(4 / 33)$ & Endoskopie $n=3$, konservativ $n=1$ \\
\hline & Mikroperforation (freie Luft) & $3 \%(1 / 33)$ & konservativ \\
\hline & Appendizitis & $12 \%(4 / 33)$ & konservativ \\
\hline & Postpolyektomiesyndrom & $12 \%(4 / 33)$ & konservativ \\
\hline \multirow[t]{4}{*}{ Major } & Appendizitis & $9 \%(3 / 33)$ & $\mathrm{OP}$ \\
\hline & Mediainfarkt rechts & $3 \%(1 / 33)$ & \\
\hline & Bridenileus & $3 \%(1 / 33)$ & 8 Monate nach Intervention, OP \\
\hline & Mesenteritis & $3 \%(1 / 33)$ & 2 Monate nach Intervention, OP \\
\hline
\end{tabular}

Schlussfolgerung Durch die EFTR kann den meisten Patienten mit Polypen am Appendixabgang eine operative Resektion erspart werden. Bei postinterventionellen Beschwerden ist der Ultraschall das diagnostische Mittel erster Wahl. Die Kenntnis der neuen Resektionstechnik inklusiver der damit einhergehenden Komplikationen ist notwendig, um Befunde richtig zu interpretieren und gegebenenfalls eine weitergehende Untersuchung oder Therapie zu veranlassen.

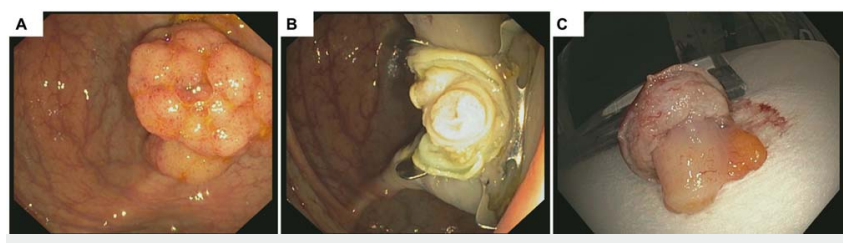

Abb. 1 


\section{10.06 Vergleich der Lebersteifigkeit im rechten und linken Leberlappen, gemessen mittels 2D-Scherwellen-Elastografie (2D-SWE), bei gesunden Probanden und Patienten mit chronischer Lebererkrankung}

\author{
Autoren Petzold $\mathrm{C}^{1}$, Grieme $\mathrm{B}^{1}$, Ellenrieder $\mathrm{V}^{1}$, Neesse $\mathrm{A}^{1}$, Kunsch $\mathrm{S}^{1}$ \\ Institut 1 Klinik für Gastroenterologie und gastrointestinale Onkologie, \\ Universitätsmedizin Göttingen \\ DOI 10.1055/s-0038-1670460
}

Einführung Die 2D-SWE ist eine etablierte Methode zur Messung der Lebersteifigkeit (LSM), die eine gute Korrelation mit dem histologischen Fibrosegrad zeigt. Empfohlen wird dabei die LSM im rechten Leberlappen von intercostal. Bei einigen Patienten ist jedoch die LSM im rechten Leberlappen aufgrund anatomischer Gegebenheiten technisch nicht möglich. Die in der Literatur definierten cut-off-Werte haben für die Messung im linken LL jedoch keine Gültigkeit. Ziel dieser Studie ist es, zu untersuchen, ob die LSM im linken Leberlappen eine gleichwertige Alternative darstellt.

Material und Methoden Es wurden gesunde Probanden ( $G P, n=36)$ und Patienten mit chronischer Lebererkrankung ( $C L D, n=36)$ prospektiv eingeschlossen. 58,3\% der Teilnehmer waren männlich. Bei allen Personen erfolgte die LSM mittels 2D-SWE (Logiq E9, GE) im rechten Leberlappen von intercostal (rLL) gemäß den Leitlinienempfehlungen sowie die Messung im linken Leberlappen von subcostal zum einen in Atemmittellage (ILLaml) und zum anderen in Inspiration (ILLins). Zusätzlich wurden der Haut-Leberkapsel-Abstand (HLA) gemessen und klinische und laborchemische Parameter erhoben.

Resultate LSM im rLL war in $87,5 \%$ der Fälle erfolgreich, im linken Leberlappen in 76,4\% (ILLaml 48,6\%, ILLins 61,1\%). Dieser Unterschied war nicht signifikant, es zeigte sich jedoch ein statistischer Trend $(p=0,08)$. Der HLA war links signifikant höher als rechts $(20,73( \pm 6,68) \mathrm{mm}$ vs. $17,11( \pm 4,20) \mathrm{mm}$; $p<0,001)$. Bei einem $H L A \geq 25$ mm waren nur $40 \%$ der Messungen erfolgreich. Bei $6,9 \%$ der Teilnehmer war die LSM nur im linken LL, jedoch nicht im rLL möglich. Sowohl in der GP-Gruppe als auch in der CLD-Gruppe waren die LSWerte im rLL signifikant niedriger als im ILLaml $(5,23( \pm 0,90) \mathrm{kPa}$ vs. 5,86 $( \pm 0,96) \mathrm{kPa} \quad(p=0,01) \quad$ bzw. $8,08 \quad( \pm 4,01) \mathrm{kPa} \quad$ vs. $13,78 \quad( \pm 8,71) \mathrm{kPa}$ $(p=0,003))$. In beiden Gruppen wurde zudem ein statistischer Trend für höhere LS-Werte in ILLins als in ILLaml beobachtet $(p=0,09$ bzw. $p=0,07)$. Bei Messung im linken LL werden unter Berücksichtigung von publizierten cut-offWerten für die Fibrosegrade 20\% der gesunden Probanden und 76,9\% der Patienten der CLD Gruppe ohne Zirrhose fälschlicherweise zu hoch klassifiziert.

Schlussfolgerung Durch Anwendung der LSM auch im linken Leberlappen kann die Anzahl technisch erfolgreicher Lebersteifigkeitsmessungen erhöht werden. Von einer unkritischen Anwendung in der klinischen Praxis wird aufgrund der Überschätzungen des Fibrosegrades jedoch dringend abgeraten.

\section{10.07 Quantitative evaluation of bowel perfusion in patients with food allergy using Dynamic Contrast-Enhanced Ultrasound (DCE-US) in comparison to healthy controls and patients with crohns disease}

Authors Wildner $D^{1}$, Raithel $\mathrm{M}^{1}$, Strobel $D^{1}$, Görtz RS ${ }^{1}$, Neurath MF ${ }^{1}$, Pfeifer $\mathrm{L}^{1}$

Institute 1 Medizinische Klinik 1, Universitätsklnikikum Erlangen, FriedrichAlexander-Universität Erlangen-Nürnberg

\section{DOI 10.1055/s-0038-1670461}

Introduction Abdominal imaging in patients with functional gastrointestinal disorders like food allergy or irritable bowel syndrome is challenging and little data is available in this field. The aim of this study was an evaluation of the bowel wall perfusion with quantitative contrast-enhanced ultrasound (DCE-US) in patients with proven food allergy under provocation diet compared to healthy controls and patients with active crohn's disease.

Material and Methods Patients with food allergy (PFA), healthy subjects (HS) and patients with active crohn's disease (PCD) scheduled for therapy escalation were included. We performed DCE-US examinations of the terminal ileum in PFA and HS after hypoallergic diet (potatoe-rise-diet - PRD) and intake of food with high allergenicity and in PCD. The following DCE-US paramters were calculated and compared: Peak Enhancement (PE), Wash-in Area Under the Curve (WiAUC), Rise Time (RT), Mean Transit Time (MTT), Time-To-Peak (TTP), Wash-in-Rate (WiR), Wash-in Perfusion Index (WiPI), Wash-out Area under the Curve (WoAUC), Wash-in Wash-out Area under the Curve (WiWoAUC), Fall Time (FT) and Wash-out Rate (WoR).

Results 37 individuals were included (14 PFA, 8 HS, 15 PCD). There was no statistical significant difference between PFA and HS, neither under PRD, nor by hyperallergic food. Amplitude related DCE-US parameters, representing the regional blood volume (PE, WiAUC, WiR, WiPi, WoR) were significantly increased in PCD compared to PFA (e.g. PE: $p=0.02$ ) and PCD compared to HS (e.g. PE: $p<0.01$ ) under hyperallergic food, indicating mural hyperemia in PCD. Time related parameters representing blood flow (RT, TTP, mTTI, FT) were consistently insignificant.

Conclusion Transmural hyperemia can be visualized by DCE-US in PCD, but not in patients with PFA and HS.

\section{Wi-Vo 11 Gefäßdiagnostik}

Fr. 16.11. 10:20 - 12:00 Shanghai 3

\section{11.01 4D/3D Farb-Doppler Sonografie der Arteria carotis interna: Validität des lokalen versus distalen Stenosegrades}

Autoren Macharzina $R R^{1}$, Kocher $S^{1}$, Messé $S R^{2}$, Hoffmann $F^{1}$, Rastan $A^{1}$, Fan $\mathrm{N}^{1}$, Vach $\mathrm{W}^{3}$, Rutkowski $\mathrm{T}^{1}$, Reuter $\mathrm{B}^{1}$, Neumann $\mathrm{FJ}{ }^{1}$, Zeller $\mathrm{T}^{1}$ Institut 1 Department of Cardiology and Angiology II, University Heart Center Freiburg-Bad Krozingen; 2 Department of Neurology, University of Pennsylvania, Philadelphia; 3 University of Freiburg, Center for Medical Biometry and Medical Informatics

DOI 10.1055/s-0038-1670462

Hintergrund Die Genauigkeit der Arteria carotis interna Stenose (ACIS) hängt von der jeweiligen Messmethode ab und beinflusst therapeutische Entscheidungen und Studienergebnisse. Die NASCET Messmethode bezieht das minimale Lumen auf den distalen Innendiameter und die ECST-Methode auf den lokalen Aussendiameter. Die direkte morphometrische Stenosemessung mit dem vierdimensional-assistierten dreidimensionalen farb-Doppler Ultraschall (4D/3DC-US) zeigte eine gute Validität für die etablierte NASCET-Methode. Die NASCET-Definition birgt klinisch relevante Nachteile. Unser Ziel war die Validität der ECST- im Vergleich zur NASCET-Methode prospektiv zu untersuchen, und die Genauigkeit im Vergleich zur klinisch etablierten Doppler-Sonografie (DUS) zu bestimmen.

Methoden In unserem Gefäßzentrum wurden 93 Patienten mit 122 ACIS mit der 4D/3DC-US Technologie untersucht. Hauptausschluskriterien waren unzulängliche Bildqualität, vorhergehende Revaskularisierung und Kontraindikationen zur Katheterangiografie. Die Studienuntersuchungen erhielten 80 Patienten mit der 4D/3DC-US (10 MHz) jeweils untereinander und zur nachfolgenden Katheterangiografie verblindet. Multiplanares off-line rendering wurde für die \%-NASCET und \%-ECST Stenose separat durchgeführt und veschiedenen Stenosegrade bestimmt.

Ergebnisse Die Spearman Korrelationskoeffizienten der Stenosegrade in der 4D/3DC-US zur Angiografie unterschieden sich nicht signifikant voneinander (NASCET 0,84, ECST 0,77, p=0,155). Die Standardabweichung der Differenzen der Stenosegrade zwischen der 4D/3DC-US und der Angiografie (SDD) war für die NASCET- $(8,1 \%)$ und die ECST-Methode $(9,1 \%)$ ähnlich gut $(p=0,241)$. Die Intermethoden SDD der ECST Methode war zu der Inter- 
observer SDD 52 konsekutiver Stenosen gemäss NASCET Definition nicht-inferior $(p=0,065)$. Ebenso zeigten sich keine Unterschiede der mit gleicher Methode gemessenen interobserver SDDs zwischen den angiografischen und den 4D/3DC-US Beobachtern ( $p \geq 0,076$ ). Binär zeigte die ECST- versus NASCET-Stenosegradbestimmung im Vergleich zur Angiografie einen nur tendenziell geringeren Kappa Wert $(0,61$ vs. 0,75$)$ bei ebenso nicht signifikant niedrigerer Sensitivität und Spezifität $\geq 85 \%(p=0,096)$. Die Genauigkeit der binären Stenosegraduierung mit der 4D/3DC-US unterschied sich nicht relevant von der DUS.

Zusammenfassung Die Anwendung der neuen 4D/3DC-US auf die ECST Methode ist im Vergleich zur NASCET-Definition eine akkurate, gut reproduzierbare Alternative und eröffnet Perspektiven für die klinische Anwendung.

\section{11.02 Clinical significance of serial duplex ultrasound in cervical artery dissection patients}

Authors Traenka $C^{1}$, Streifler $]^{2}$, Lyrer PA ${ }^{1}$, Engelter $\mathrm{ST}^{1,3}$

Institute 1 Department of Neurology and Stroke Center, University Hospital Basel and University of Basel, Basel; 2 Department of Neurology, Rabin Medical Center, Petah Tikva; the Sackler Faculty of Medicine,Tel Aviv

University; 3 Neurorehabilitation Unit, University of Basel and University Center for Medicine of Aging, Felix Platter Hospital, Basel

DOI 10.1055/s-0038-1670463

Objective To assess the frequency of primary and delayed occlusion, recanalization of the dissected artery as well as recurrent artery dissection as detected in serial color-coded duplex ultrasound (DUS) in routine clinical care and follow-up of Cervical Artery Dissection (CeAD) patients.

Methods Retrospective review of serial DUS exams performed at baseline and follow-up during clinical routine in consecutive CeAD patients at the Stroke Center Basel, Switzerland. Examinations were reviewed by a single rater - not involved in clinical care of the patients - for the presence of (i) mural hematoma, (ii) intimal flap, (iii) hemodynamics - in particular occlusion - of the dissected artery at baseline and during routine follow-up. Findings were correlated to clinical events.

Results In 96 CeAD patients ( $n=42$ female (41.6\%) 508 DUS examinations were performed and reviewed in this study. Median age at baseline was 46 years (IQR 36-53). Median time from baseline DUS to last DUS follow-up exam was 12 months (IQR 7 - 23)). In baseline DUS exams, mural hematoma and intimal flap were seen in 41 (38.3\%) and 11 (10.3\%) of arteries, respectively. Occlusion of the dissected artery was seen in 27 (25.2\%) in baseline DUS. At follow-up 1, partial or complete recanalization of the occluded artery was seen in 15 (of 27 (55.5\%)) cases of which three were clinically symptomatic with new ischemic or local symptoms. Secondary occlusion occurred in 3 (of $96(3.1 \%)$ ) CeADs, all of which were clinically asymptomatic and were detected during routine, pre-scheduled follow-up. Five (5.2\%) patients had recurrent dissection, all of which were clinically symptomatic.

Conclusion Color-coded duplex ultrasound is a useful, widely available tool to monitor CeAD patients in routine clinical care and follow-up. Hemodynamic changes as wells as the occurrence of new dissections - either clinically symptomatic or asymptomatic - can be detected by DUS, providing clinically relevant information, which may guide individual treatment decisions.

\section{11.03 Intraoperative Kontrastmittelsonografie zur Detektion von Endoleaks nach endovaskulärer Aortenaneurysmaausschaltung}

\footnotetext{
Autoren Keschenau $\mathrm{PR}^{1}$, Jacobs MJ ${ }^{1}$, Kalder J

Institut 1 Universitätsklinikum Aachen

DOI 10.1055/s-0038-1670464

Einführung Die Kontrastmittelsonografie (CEUS) gehört aufgrund verschiedener Vorteile (fehlende Nephrotoxizität, lod- und Strahlenbelastung) zu den Routineverfahren in der Verlaufskontrolle nach endovaskulärer Aortenaneurysmaausschaltung (EVAR). Ziel dieser Pilotstudie war die Evaluation der intra-
}

operativen CEUS (iCEUS) zur Detektion von Endoleaks unmittelbar nach Implantation aortaler Endoprothesen.

Material und Methoden Es wurden 13 Patienten prospektiv eingeschlossen, die zwischen Oktober 2016 und November 2017 eine EVAR mit primärer Komplettierung der Prozedur erhielten. Bei 2 Patienten wurden infrarenale Endoprothesen (Medtronic Inc, Santa Rosa Calif) implantiert, die übrigen Patienten erhielten fenestrierte/gebranchte Endoprothesen der Firma Cook (Australia Pty. Ltd., Brisbane; $n=5$ ) oder Vaskutek (Glasgow, Scotland, Großbritannien; $n=6$ ). In einem Fall wurde zusätzlich beidseits eine lliac side branch Prothese (Fa. Cook) implantiert. Bei allen Patienten wurde im unmittelbaren Anschluss an die Abschlussangiografie eine Kontrastmittelsonografie mittels eines Toshiba TUS-X200 (Toshiba Medical Systems, Zoetermeer, The Netherlands) Ultraschallgeräts durchgeführt. Hierzu wurden je $2 \mathrm{ml}$ Kontrastmittel (SonoVue ${ }^{\circledR}$, Bracco, Milano, Italien) gefolgt von einem $10 \mathrm{ml}$ Bolus ( $\mathrm{NaCl} 0,9 \%$ injiziert. Alle Patienten erhielten vor Entlassung aus der stationären Behandlung eine CT-Angiografie (CTA), die mit den intraoperativen Ergebnissen verglichen wurde.

Ergebnisse In der Abschlussangiografie wurden insgesamt 5 Typ 2 Endoleaks nachgewiesen, wobei in 4 Fällen der Ursprung nicht sicher zu beurteilen war. Die CEUS wies diese 5 Endoleaks ebenfalls nach, sowie zusätzlich 4 weitere Typ 2 Endoleaks, welche nicht in der Angiografie zur Darstellung gekommen waren. Bei allen Endoleaks konnte der Ursprung mittels CEUS nachgewiesen werden: A. mesenterica inferior (AMI; $n=7$ ), Lumbalarterie $(L A ; n=1)$, Kommunikation AMI/LA $(n=1)$. In der CTA vor Entlassung persistierten nur 2 der intraoperativ festgestellten Endoleaks, von denen eines initial nur mittels CEUS detektiert worden war. In einem Fall mit intraoperativ unauffälliger Angiografie und CEUS zeigte die CTA ein neu aufgetretenes Typ 3 Endoleak.

Schlussfolgerung Die iCEUS scheint eine höhere Sensitivität als die Angiografie zur Detektion von Endoleaks unmittelbar nach EVAR zu haben. Zudem ist mittels CEUS eine zuverlässige und präzise Angabe des Endoleakursprunges möglich. Die meisten intraoperativen Typ 2 Endoleaks verschließen sich spontan während des stationären Aufenthaltes.

\section{11.04 Fokussierte sonografische Differenzialdiagnose der unklaren Leistenschwellung}

Autoren Nöldeke $S^{1}$

Institut 1 Klinik für Gefäßchirurgie, Klinikum Garmisch-Partenkirchen DOI 10.1055/s-0038-1670465

Problemstellung Die Abklärung von unklaren Leistenschwellungen ist eine häufige Fragestellung in einer gefäßchirurgischen Ambulanz. Die möglichen Differenzialdiagnosen reichen vom einfachen Aneurysma spurium über komplexe Aneurysmata mit oder ohne vaskuläre Vor-Therapie, Patienten mit Gefäßverletzungen bis hin zu nicht primär vaskulären Ursachen wie Leistenhernie, Lymphknotenpathologien oder echte tumoröse Raumforderungen.

Material und Methode Von 2008 bis 2018 wurden 149 Patienten, die mit der Diagnose „unklare Leistenschwellung“ vorstellig wurden, an einem High-endGerät untersucht und jeweils eine klare diagnostische Zuordnung getroffen. Ergebnisse Folgende Ursachen konnten festgestellt werden: Aneurysma spurium nach Intervention oder Operation (65), primäres arterielles Aneurysma (13), sekundäres arterielles Aneurysma (26), venöses Aneurysma (3), Gefäßverletzung (5), Serom oder Lymphozele (15), Infekte nach Gefäßoperationen (8), Hüftgelenkszyste (3), Leistenhernie (5), Lymphknotenschwellungen (3) und echte Weichteiltumore (3). In lediglich fast 9 Prozent) musste eine weitere Diagnostik (CT) erfolgen. Dies waren erstens die Patienten mit einer infizierten Gefäßprothese, wo das Ausmaß der abdominellen Beteiligung präoperativ geklärt werden musste. Die zweite Gruppe waren die Patienten mit V.a. Weichteiltumor in der Leiste, wo sich retrospektiv ein Sarkom (unbekannt) und 2 Lymphome (eine Grunderkrankung unbekannt) ergaben.

Diskussion Die Bedeutung der Sonografie für Abklärungen von unklaren Schwellungen der Leistenregion ist unbestritten und belegt. Allerdings zeigen die Ergebnisse aus unserer Gefäßambulanz, dass hier keineswegs fachspezi- 
fisch „gesucht“ werden darf sondern auch relevante Krankheitsbilder anderer Fachdisziplinen sowohl gekannt als auch erkannt werden müssen. Die Entscheidung, ob ein rein vaskuläres Problem vorliegt ließ sich mit Duplex-, Farbduplex- und B-Flow-Techniken immer zweifelsfrei klären. Ebenso war die Frage einer sofortigen Intervention z. B. bei einem Aneurysma spurium ohne weitere Untersuchungen zweifelsfrei zu klären. Eine weitergehende Schnittbilddiagnostik war nur in wenigen Fällen (8,7 Prozent) erforderlich, was die Bedeutung einer fokussierten Sonografie der Leistenregion unterstreicht. Im Rahmen von Ultraschall-Kursen und -weiterbildungen sollte das Thema wegen der Häufigkeit und der zu beachtenden Differenzialdiagnostik gesondert betrachtet und auch anhand von Videofiles unterrichtet werden.

\section{11.05 Evaluation der Flusscharakteristik in der abdominellen Aorta und Nierenarterien mittels einer plane wave Technik}

Autoren Weskott $\mathrm{HP}^{1}$

Institut 1 Ultraschall Ambulanz Klinikum Siloah, KRH Hannover DOI 10.1055/s-0038-1670466

Einführung Mittels der Plane Wave Technik lassen sich Frame rates von 600 700fps bei wechselnder Angulierung der Schallwellenfront in Form von farbigen Pfeilen darstellen.

Material und Methoden. Gerät FKDS und Vektorflussanalyse (V-Flow) mit der L9 $3 \mathrm{U}$ Linearsonde (Mindray, Resona 7). Untersucht wurde die Aorta und rechte Nierenarterie bei 15 schlanken meist jungen Patienten mit benignen hepatischen Läsionen in der Spätphase nach SonoVue Bolusgabe von 1,4 ml. Die akustische Sendeleistung betrug 10\%.

Resultat In der Aorta wird der spirale Flusscharakter mit hoher Orts- und Richtungserkennung dargestellt. Die Flussrichtung in der Aorta ändert sich in Abhängigkeit vom kardialen Zyklus über eine relativ uniforme Flussrichtung in der frühen Systole gefolgt von einem spiralen Flussmuster über die ganze Länge der Aorta. In der frühen bis mittleren Systole fließt das Blut in Höhe der Nierenarterienabgänge häufig ventralwärts. Daraus folgt eine kurze midsystolische Wirbelbildung am Abgang der rechten Nierenarterie, die bei 10/15 Patienten dokumentiert werden wurde. Bei 5 Patienten konnte kein abgangsnaher Wirbel dargestellt werden, was in 3/5 an einer nicht gut angepassten Kontrastmittelkonzentration oder an einer zu tiefen Lage der NA lag. Schlussfolgerung Ein Spiralfluss als physiologische Flussmuster konnte mittels plane wave imaging Technik mit hoher Orts- und Zeitauflösung in der Aorta sowie erstmals auch eine Wirbelbildung am Abgang der rechten Nierenarterie nachgewiesen werden.

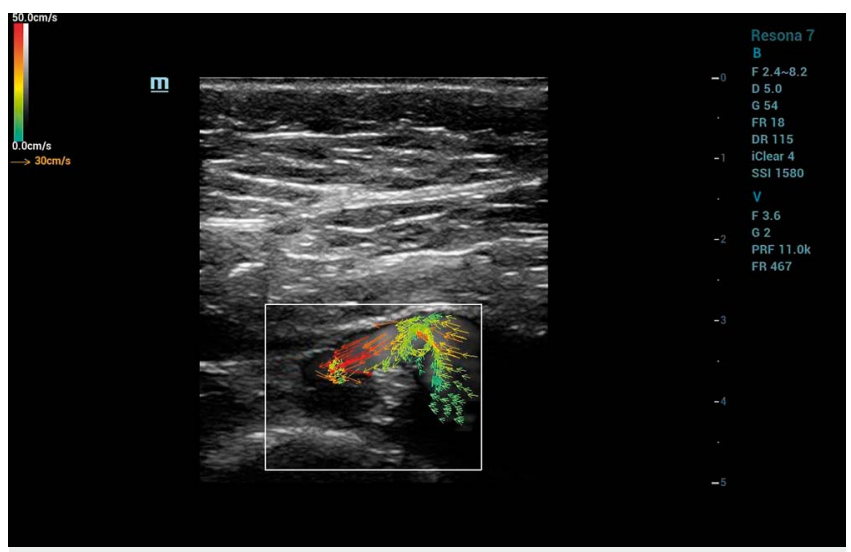

Abb. 1

\section{11.06 Ressourcen sparendes effektives Ausbildungskonzept am Beispiel der TVT-Diagnostik}

Autoren Krishnabhakdi S

Institut 1 Gefäßchirurgie, Klinikum Herford

DOI 10.1055/s-0038-1670467

Einführung Die Sonografie ist ein klinisch apparatives Verfahren, dass dem jungen Mediziner den Einstieg in die klinische Medizin erleichtern kann. Vor dem Hintergrund knapper werdender Ressourcen wird ein zeitsparendes Ausbildungskonzept am Beispiel der Kompressionssonografie (Thrombosediagnostik) vorgestellt.

Material und Methoden 2 Untersucher, 1 DEGUM-Ausbilder pro Gerät. Linearscan, 5-7,5 MHz. Gesunde und Thrombose (TVT) Patienten mit BMI von maximal 30, TVT Alter unter 2 Wochen. 14 Medizinstudenten (>8. Klinisches Semester) wurden in einem Pilotprojekt nach einem Stufenkonzept ausgebildet: Physik auf 4 Folien interaktiv - Übung Schallkopforientierung - 3x Kompressionssonografie am Bein (Leiste, Oberschenkel, Kniekehle) - 3x Kompressionssonografie am Bein ( $V v$. gastrocn, m. solei, tibialis anterior, posterior und fibularis) - 3x TVT Patienten (femoropopliteal). Nach einem Durchgang des Stufenkonzepts wurde der Lernerfolg an 10 TVT Patienten überprüft. Resultate

1. TVT sicher diagnostiziert: VFC 14/14, VFS 13/14, V pop 14/14, VV. gastrocnemiae 10/14, V m. solei 4/14, VTP 10/14, V fib 7/14, VTA 8/14.

2. Die Lernmotivation und Zufriedenheit konnte durch den umsetzbaren Lernerfolg auf verschiedenen Ebenen gesteigert werden.

3. Ausbildern entstanden Freiräume für andere Tätigkeiten.

Schlussfolgerung Die Ergebnisse weisen darauf hin, dass insbesondere in der femoropoplitealen Etage eine gestraffte, zielorientierte Ausbildung bereits in einem frühen Stadium der Ausbildung ausreichen könnte, um bei nicht adipösen Patienten zuverlässig eine TVT -Diagnostik durchzuführen. Die Ergebnisse werden mit der Literatur zu sonografischen Ausbildungskonzepten auch anderer Entitäten verglichen. Die Auswirkungen auf frei werdende Ressourcen und das Bewerbungsverhalten werden beschrieben.

\section{11.07 Fokussierte sonografische Differenzialdiagnostik der Kniekehlenschwellung}

\section{Autoren Nöldeke $S^{1}$}

Institut 1 Klinik für Gefäßchirurgie, Klinikum Garmisch-Partenkirchen DOI 10.1055/s-0038-1670468

Problemstellung Die Abklärung von poplitealen Schwellungen ist ein nicht seltenes Problem in einer gefäßchirurgischen Ambulanz. Die möglichen Differenzialdiagnosen reichen von der einfachen über die komplexe popliteale Gelenkzyste, das primäre Popliteaaneurysma, postoperative liquide Raumforderungen oder Blutungen, iatrogene oder traumatische Verletzungen bis hin zu seltenen Ursachen tropischer Genese.

Material und Methode Von 2008 bis 2018 wurden alle 94 Patienten, die mit der Diagnose „Kniekehlenschwellung“ vorstellig wurden, an jeweils einem High-end-Gerät (GE Logiq E9) untersucht und jeweils eine klare diagnostische Zuordnung getroffen. Eingeschlossen waren unter Mitbetreuung einer großen Unfallchirurgie auch (Poly-)Trauma-Patienten und Patienten einer high-volume-Endoprothetik.

Ergebnisse Die Sonografie der Poplitealregion bietet vom Zugang her keine Probleme, allerdings müssen je nach Eindringtiefe u.U. nicht nur Linear- sondern auch niederfrequente Curved-Schallköpfe eingesetzt werden. Folgende Ursachen für eine primär unklare Kniekehlenschwellung fanden sich: Am häufigsten waren Gelenkzysten (34), gefolgt von echten Poplitea-Aneurysmata (26). Bei einem hohen Anteil an Patienten nach Endoprothese oder Arthroskopie fanden sich eine 15 Patienten mit einer Einblutung. Weitere Diagnosen waren venöse Ektasien bzw. variköse Konvolute (8), Gefäßverletzungen (3), Serome oder Lymphozelen (2), Spontanblutungen in Gelenk oder Muskulatur (5). Dass man auch den Blick auf andere Fachgebiete wenden muss, zeigte 
eine Patientin, die mit geschwollenem Knie von einer Reise zurückkehrte. Hier fand sich eine kutane Wurmerkrankung als seltene Ursache.

Diskussion Die Bedeutung der Sonografie für Abklärungen von unklaren Schwellungen der Kniekehlenregion ist belegt, allerdings meist nur fachspezifisch untersucht. Allerdings zeigen die Ergebnisse aus unserer Gefäßambulanz, dass hier keineswegs fachspezifisch "gesucht" werden darf sondern auch relevante Krankheitsbilder anderer Fachdisziplinen sowohl gekannt als auch erkannt werden müssen. Die Entscheidung, ob ein rein vaskuläres Problem vorliegt ließ sich mit Ultraschall immer zweifelsfrei klären, wobei unter Zuhilfenahme sensitiver Flussdetektion fokussiert differenzialdiagnostisch gesucht werden muss. Eine weitergehende Schnittbilddiagnostik war insgesamt nur selten erforderlich, was die Bedeutung einer fokussierten Sonografie der Poplitealregion unterstreicht.

\section{Wi-Vo 12 Gynäkologie/Geburtshilfe V}

Fr. 16.11. 13:00 - 14:30 Shanghai 2

\section{12.01 Wertigkeit von international standardisierten Wachstumskurven (INTERGROWTH-21st) bei Feten mit Hypoplastischen Linksherzsyndrom: Perinatale Biometrie und Dopplersonografie sowie kurzfristiges Outcome}

Autoren Graupner $\mathrm{O}^{1}$, Helfrich $\mathrm{F}^{1}$, Wacker-Gussmann $\mathrm{A}^{2}$, Ostermayer $\mathrm{E}^{1}$, Lobmaier $\mathrm{SM}^{1}$, Ortiz JU $\mathrm{U}^{1}$, Haller $\mathrm{B}^{3}$, Oberhoffer $\mathrm{R}^{4}$, Kuschel $\mathrm{B}^{1}$

Institut 1 Frauenklinik und Poliklinik, Klinikum rechts der Isar; 2 Klinik für Kinderkardiologie und angeborene Herzfehler, Deutsches Herzzentrum

München; 3 Institut für Medizinische Informatik, Statistik und Epidemiologie; 4 Zentrum für Präventive Pädiatrie/ 1 - 4 Technische Universität München DOI 10.1055/s-0038-1670469

Einführung Kinder mit Hypoplastischen Linksherzsyndrom (HLHS) weisen häufig ein niedrigeres Geburtsgewicht (GG) und einen niedrigeren postnatalen Kopfumfang (KU) auf. Beide Parameter sind bedeutsam für die Überlebensrate und das neurologische Outcome betroffener Kinder. Bislang publizierte Arbeiten beziehen sich auf eigene Wachstumskurven, sodass eine Vergleichbarkeit der Resultate erschwert ist. Ziel dieser Studie ist nun die Anwendung international standardisierter Wachstumskurven bei Feten mit HLHS.

Material und Methoden Es handelt sich um eine retrospektive monozentrische Studie. Mehrlingsschwangerschaften, Fälle mit Schwangerschaftsabbruch und/oder nachgewiesenen Chromosomenaberrationen wurden ausgeschlossen. Für die z-Score Berechnung der prä- (KU und fetales Schätzgewicht: EFW) und postnatalen (KU und GG) Biometrie-Werte wurde der INTERGROWTH-21st [1] Kalkulator verwendet. Ebenso wurden Daten zur fetalen Dopplersonografie (Pulsatilitätsindex der Umbilikalarterie: UA PI, PI der mittleren Zerebralarterie: ACM PI, cerebroplazentare Ratio: CPR) sowie zum kurzfristigen perinatalen Outcome erhoben.

Resultate Es konnten 49 HLHS-Feten (132 Untersuchungen) eingeschlossen werden. Das mittlere Schwangerschaftsalter zum Zeitpunkt der Untersuchung betrug $35 \pm 3,4$ SSW. Für den pränatalen KU ergab sich ein z-Score von -0,391 (95\%-KI: $-0,716$ bis $-0,066 ; p=0,021$ ) und für das EFW ein $z$-Score von $-0,023$ (95\%-KI: $-0,277$ bis 0,229; $p=0,853$ ). Das mittlere Schwangerschaftsalter zum Zeitpunkt der Geburt betrug 38,4 $\pm 1,0$ SSW mit einem durchschnittlichen GG von $3032 \pm 402 \mathrm{~g}$ ( $\mathrm{z}$-Score: $-0,209 \pm 0,861 ; \mathrm{p}=0,107)$ und einem durchschnittlichen $\mathrm{KU}$ von 33,6 $\pm 1,5 \mathrm{~cm}$ ( $z$-Score: 0,133 $\pm 1,103$; $\mathrm{p}=0,430$ ). Die 30 TagesÜberlebensrate betrug 73,3\% (33/45). Dabei zeigte sich kein signifikanter Unterschied in den z-Scores zwischen den verstorbenen (Gruppe 1) und überlebenden (Gruppe 2) HLHS-Kindern. Es konnte keine signifikante Korrelation zwischen dem UA PI, ACM PI sowie der CPR und dem GG bzw. postnatalen KU in beiden Gruppen beobachtet werden.

Schlussfolgerung Wir konnten mittels INTERGROWTH-21st zeigen, dass die Verteilung des EFW bei den beobachteten HLHS-Kindern nahe an der für ein Normalkollektiv erwarteten Verteilung liegt, der KU jedoch tendenziell gerin- ger ausfällt. Diese Beobachtung konnte postnatal nicht bestätigt werden. Eine Assoziation der biometrischen Maße mit der 30-Tagesüberlebensrate ließ sich nicht feststellen.

1. https://intergrowth21.tghn.org Stand: 17.05 .2018

\section{12.02 Vergleich von studententutorbasierte und ausschließlich ärztlich geleitete geburtshilfliche und gynäkologische Ultraschallbasiskurse in der Medizinlehre am UKS}

Autoren Hamza $A^{1}$, Radosa $J^{1}$, Takacs $Z^{1}$, Solomayer $\mathrm{EF}^{1}$, Ströder $\mathrm{R}^{1}$, JuhaszBoess $I^{1}$, Meyberg-Solomayer $\mathrm{B}^{1}$

Institut 1 Klinik für Frauenheilkunde, Geburtshilfe und

Reproduktionsmedizin, Universitätsklinikum des Saarlandes

DOI 10.1055/s-0038-1670470

Einführung Der Einsatz von Studententutoren in der Lehre wird lange diskutiert. Im Hinblick der Sicherheit der Lehre erfolgte im Bereich der Frauenheilkunde kein Vergleich zwischen eines ausschließlich ärztlich geleiteten und eines studententutorenbasierten Ultraschallkurses.

Material und Methoden Wir haben den Inhalt eines Ultraschallkurses im Bereich Frauenheilkunde standardisiert. Zwischen Oktober 2014 und Januar 2015 hat der ausschließlich ärztlich geleitete Ultraschallkurs stattgefunden. Daraufhin folgte der studententutorenbasierte Kurs zwischen April bis Juli 2017. Die Studenten haben in beiden Kursen während ihres Blockpraktikums in der Gynäkologie und Geburtshilfe einen identischen theoretischen und praktischen Ultraschallkurs mit Hands-on erhalten. Die theoretischen Kenntnisse und fetale morphologische Merkmale wurden vor und nach dem Kurs mittels denselben Mutliple-Choice-Fragen überprüft. Nach dem Kurs haben die Studenten den Kurs mittels einen identischen Evaluationsbogen ausgefültt ausgewertet.

Resultate 109 Studenten haben am ärztlich geleiteten Kurs teilgenommen. 111 Studenten haben an studententutorebasierten Kurs teilgenommen. Die Resultate der Posttestate wurden verglichen. Im theoretischen Teil der Prüfung haben die Blockstudenten aus dem Studententutorenkurs statistisch signifikant abgeschnitten ( $100 \%$ vs. $75, \mathrm{p}<0,05)$. Im Praktischen Anteil der Prüfung gab es keinen statistischen relevanten Unterschied (83\% vs. $83 \%$, n. s.). In der gesamten Prüfung haben die Blockstudenten aus dem Studententutorenkurs insgesamt besser abgeschnitten ( $90 \%$ vs. $80 \%, p<0,05)$. Aus dem Evaluationsbogen ergab sich eine allgemeine Zufriedenheit der Studenten, subjektiv die Einschätzung einer Kompetenzerweiterung und die Forderung nach mehr sonografischem Hands-on Training im Gebiet der Geburtshilfe und Gynäkologie sowie in anderen Bereichen der Medizin. Die Likert-Werte der Blockstudenten aus dem Studententutorenkurs waren statistisch signifikant besser.

Schlussfolgerung Sowohl objektiv im Rahmen der MCQ Prüfung als auch subjektiv im Rahmen des Evaluationsbogen zeigte der Einsatz der Studententutoren im Blockpraktikum eine qualitative Verbesserung der Lehre. Wir empfehlen daher den Einsatz der Studententutoren zu verstärken.

\section{12.03 Anwendbarkeit der FMF-Qualitätskriterien für das Ersttrimesterscreening in einem unselektierten Patientinnenkollektiv}

Autoren Ritgen J ${ }^{1}$, Stressig R ${ }^{1}$, Weichert J ${ }^{2}$, Hartge DR ${ }^{2}$, Degenhardt J ${ }^{1}$ Institut 1 Praenatalplus, Praxis für Praenatale Medizin und Genetik, Köln; 2 Abteilung für Pränatalmedizin, Klinik für Frauenheilkunde und Geburtshilfe, Universitätsklinikum Schleswig-Holstein, Lübeck

DOI 10.1055/s-0038-1670471

Einführung Im Rahmen der Ersttrimester-Diagnostik sind neben der Messung der fetalen Nackentransparenz (NT) eine zunehmende Zahl weiterer Qualitätsund Screeningparameter in der Untersuchungsroutine etabliert. Ziel der Untersuchung war es, die Anwendbarkeit streng nach Kriterien der FMF London 
in der klinischen Alltagssituation in einem unselektierten Screeningkollektiv zu evaluieren.

Material und Methoden In unseren Einrichtungen werden jährlich zusammen etwa 1800 Erstrimesterscreenings (ETS) durchgeführt. Eine Stichprobe aus dem Zeitraum Dezember 2017 bis Januar 2018 wurde retrospektiv untersucht. Dazu wurde aus jeder Untersuchung eine Abbildung ausgewählt, die die höchste Qualität nach den FMF-Zertifizierungsrichtlinien aufwies. Alle Untersuchungen wurden transabdominal von Untersuchern der DEGUM-Stufen II und III mit aktuellen High-End Ultraschallgeräten (GE Voluson E10, Toshiba Aplio 900, Philips Epiq 7) durchgeführt. Die Abbildungen wurden auf die Qualität folgender Parameter hin untersucht: Vergrößerung, neutrale Haltung des Feten, Caliberposition, thin nuchal membrane, Hirnstamm, 4. Ventrikel, Cisterna magna, Maxilla, Nasenbein, Thalamus sauber abgegrenzt, Jochbein nicht sichtbar und Insonationswinkel.

Resultate 248 ETS wurden im Untersuchungszeitraum durchgeführt. Der maternale BMI betrug im Mittel $25(17-42) \mathrm{kg} / \mathrm{m} 2$. Bei der NT-Messung wurde die Nackenhaut zu 100\% korrekt abgebildet, die Caliper-Position war in $93 \%$ adäquat („on-on“), die fetale Haltung in $85 \%$ neutral. Der Insonationswinkel war in $90 \%$ korrekt. Das Nasenbein war in $92 \%$ der Fälle sicher beurteilbar, die Maxilla in $83 \%$. Zur Beurteilung der Neuroanatomie wurden der IV. Ventrikel, die Cisterna magna, der Hirnstamm und die Thalamie dargestellt (85\%, 80\%, $81 \%, 73 \%$ ). In $80 \%$ war das Jochbein (korrekterweise) nicht abgebildet, FalxFlash war in $70 \%$ darstellbar.

Schlussfolgerung Bei idealen und guten Untersuchungsbedingungen lassen sich die FMF-Qualitätskriterien mit großer Sicherheit darstellen. Limitierender Faktor ist dabei v.a. der maternale BMI. Eine korrekte Evaluation der frühen fetalen Neurosonografie bildet im Rahmen der Untersuchung die technisch größte Herausforderung.

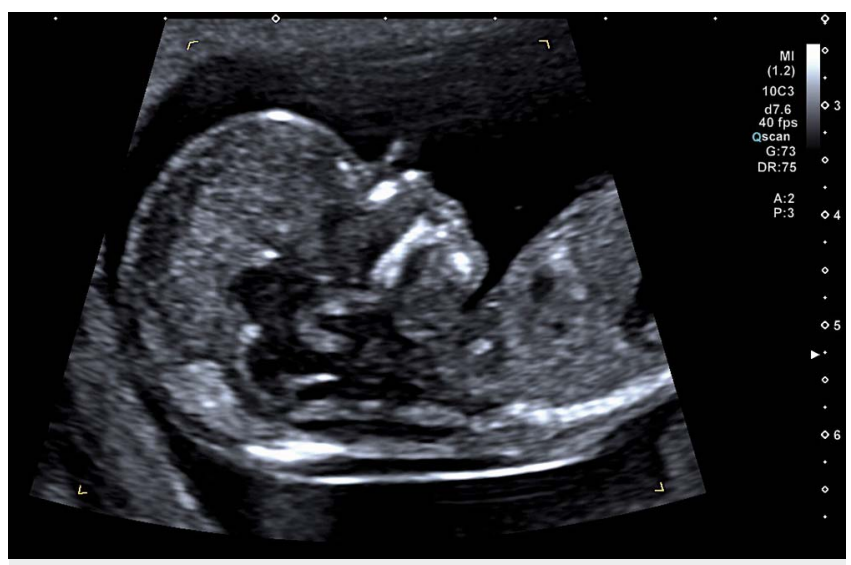

Abb. 1

\section{12.04 Evaluation der rechtsventrikulären myokardialen Kontraktilität bei Feten mit Hypoplastischem Linksherz mittels 2D Speckle Tracking}

Autoren Enzensberger $C^{1}$, Fischer $S^{1}$, Graupner $\mathrm{O}^{2}$, Götte $\mathrm{M}^{1}$, Wolter $\mathrm{A}^{1}$, Müller $\mathrm{V}^{1}$, Herrmann $\mathrm{J}^{3}$, Axt-Fliedner $\mathrm{R}^{1}$

Institut 1 Klinik für Geburtshilfe und Gynäkologie, Abteilung für Pränatalmedizin, Justus-Liebig Universität, Gießen; 2 Klinik für Geburtshilfe und Gynäkologie, Klinikum rechts der Isar, Technische Universität München; 3 Statistikberatung Gießen

DOI 10.1055/s-0038-1670472

Einführung Das Outcome von Patienten mit Hypoplastischem Linksherz (HLH) bezüglich Langzeit-Morbidität sowie -Mortalität wird erheblich durch die rechtsventrikuläre Funktion beeinflusst. Ziel dieser Studie war es, die Hy- pothese eines pränatalen Remodelings des rechten Ventrikels (RV) mit einer dopplerunabhängigen Methode wie dem Speckle Tracking zu bestätigen.

Material und Methoden Es wurde eine retrospektive Studie durchgeführt. Die fetale Echokardiografie wurde mit einem Toshiba Aplio 500 System generiert. Die Filmsequenzen wurden basierend auf einem Vierkammerblick des fetalen Herzens in der originalen Bildrate (acustic frame rate: AFR) gespeichert. Unter Verwendung der 2D Speckle Tracking Technik bestimmten wir die globalen longitudinalen Peak-Strain-Werte (LPSS) des RV und verglichen diese mit LPSS Werten des RV von gestational age matched Kontroll-Feten.

Resultate Eingeschlossen wurden 18 HLH Feten und 103 gesunden Kontrollen. Das Gestationsalter war in beiden Gruppen gleichermaßen verteilt. Die AFR betrug 118,3 \pm 20,9 Bilder pro Sekunde (bps) in der HLH Gruppe und 127 \pm 26 bps in der Kontrollgruppe. Die Feten mit HLH zeigten im Vergleich zu der Kontrollgruppe signifikant höhere globale LPSS Werte für den RV (-16,22\% vs. $-12,31 \%)$.

Schlussfolgerung Im Falle von HLH unterliegt der RV bereits pränatal einem Remodeling, welches zu einer Anpassung der myokardialen Funktion auf linksventrikuläre Bedingungen führt. In Zukunft sollen weitere Untersuchungen mittels Speckle Tracking das Wissen über die rechtsventrikuläre Kontraktilität bei HLH Feten erweitern.

\section{12.05 Unterschiede im Ductus venosus Fluss bei Feten mit Trisomie 21 mit und ohne kongenitale Herzfehler im Ersttrimesterscreening}

Autoren Wagner $\mathrm{P}^{1}$, Prodan $\mathrm{N}^{1}$, Abele $\mathrm{H}^{1}$, Hoopmann $\mathrm{M}^{1}$, Kagan $\mathrm{KO}^{1}$ Institut 1 Pränataldiagnostik, Universitätsfrauenklinik Tübingen DOI 10.1055/s-0038-1670473

Einleitung Ziel der Studie war es zu untersuchen, ob sich der Ductus venosus Fluss (DVF) bei Trisomie-21-Feten mit und ohne kongenitalen Herzfehler unterscheidet und ob dies die Risikoverteilung im Ersttrimesterscreening für Trisomie 21 (T21) beeinflusst.

Methodik Eingeschlossen in diese retrospektive Studie wurden Schwangere, die ein Ersttrimesterscreening an der Universitätsfrauenklinik durchführen ließen. Die Untersuchung beinhaltet dabei die Beurteilung der Scheitel-SteißLänge, der Nackentransparenz (NT), dem DVF und des Herzens. Es wurden drei Gruppen von Feten untersucht: euploide Feten mit unauffälliger NT und ohne Herzfehler sowie Feten mit T21 mit und ohne Herzfehler. Die Beurteilung des DVF beinhaltete den DV Pulsatility Index für Venen (DV PIV), die Richtung der a-Welle und verschiedene Ratios aus v- und a- sowie S- und DWelle (v/S, v/D, a/S, a/D, S/D).

Ergebnis Die Studienpopulation bestand aus 410 euploiden Feten und 136 Feten mit Trisomie 21, davon 51 mit und 85 ohne Herzfehler. In diesen 3 Gruppen lag ein „reverse flow“ der a-Welle in 3,2\%, 66,7\% und 57,6\% vor. Die DV-Ratios aus v- und a- sowie S- und D-Welle waren bei T21-Feten mit und ohne Herzfehler signifikant unterschiedlich zu der Gruppe mit euploiden Feten. Im Vergleich der beiden T21-Gruppen mit und ohne Herzfehler waren lediglich die a/S- und a/D-Ratios signifikant unterschiedlich. Die Risikoverteilung im Screening auf Trisomie 21 mit und ohne Herzfehler war dabei unverändert.

Schlussfolgerung Die Beurteilung des DVF ist nützlich im Screening auf T21 und auf Herzfehler. Der DV-Fluss von T21-Feten mit und ohne Herzefehler ist zwar ebenfalls unterschiedlich, aber diese Unterschiede sind zu gering, um klinische Unterscheidung zuzulassen. 


\section{12.06 Große AV-Malformation der Leber, prä- und postnataler Verlauf}

Autoren Takacs FZ1 ${ }^{1}$, Abdul-Khaliq $\mathrm{H}^{2}$, Hamza A ${ }^{1}$, Meyberg-Solomayer $\mathrm{G}^{1}$ Institut 1 Klinik für Frauenheilkunde, Geburtshilfe und

Reproduktionsmedizin; 2 Klinik für Pädiatrische Kardiologie/ 1 - 2

Universitätsklinikum des Saarlandes

DOI 10.1055/s-0038-1670474

Patienten und Methode Vorstellung einer 20-jährigen Erstgravida in der 23+0 SSW zur Feindiagnostik. Es zeigte sich eine $42 \times 41 \times 30 \mathrm{~mm}$ große arteriovenöse Malformation (AVM), die den gesamten linken Leberlappen ausgefüllt hat, mit konsekutiver Kardiomegalie (CTR 0,64) und milder Tricuspidalinsuffizienz. Der Zufluss erfolgte über die A. hepatica sinistra und V. umbilicalis, die venöse Drainage über eine massiv dilatierte Lebervene in den rechten Vorhof. Im Verlauf zeigte die AVM nur geringfügiges Wachstum, die CTR war konstant, ohne weitere Dekompensationszeichen. Außer einen leicht erhöhten umbilicalen Widerstand (RI: 0,77) war der fetale Flow normal. Die Entbindung erfolgte per primärer Sectio (3360 g, Apgar-Werte: 7/8/9, Nabelschnurarterien-pH: 7,328). Das Neugeborene zeigte eine respiratorische Insuffizienz mit Rechtsherzbelastung. Darauf erfolgte am 4. Lebenstag eine Herzkatheteruntersuchung bei der sich die ausgedehnte AV-Malformation der Leber bestätigte. Der Befund nahm die Segmente 2 und 3 fast komplett ein, mit einem Zufluss über den Truncus coeliacus und $\mathrm{A}$. hepatica sinistra und Abfluss über die mittlere und die linke Lebervene. Wegen Zeichen einer pulmonalen Hypertonie und high output Herzversagen wurde zweimal eine Embolisation mittels Onyx durchgeführt. Postinterventionell zeigte sich eine deutliche kardiale Erholung. Der Befund bildete sich bis zum 11. Lebensmonat fast komplett zurück, Herz- und Leberfunktion normalisierten sich ebenfalls. Das Kind zeigte im Verlauf eine normale Entwicklung.

Schlussfolgerung Die hepatische arteriovenöse Malformation (AVM) ist eine seltene vaskuläre Entwicklungsstörung, die durch eine direkte arterielle Verbindung zu einem fistulösen venösen Drainagesystem in der Leber gekennzeichnet ist. Der Zustand führt zu einem Shunt mit hohem Fluss und niedrigem Widerstand, der high output Herzversagen und Hydrops bereits vorgeburtlich verursachen kann. Eine pränatale Diagnose ist mithilfe der Dopplersonografie möglich. Zu den aktuellen Behandlungsoptionen gehören die Obliteration der arteriellen Versorgungsgefäße mit perkutaner Transkatheterembolisation, die in der frühen postnatalen Phase auch durchgeführt werden kann, oder die chirurgische Ligatur.

\section{12.07 Fetale Sinusthrombose - 2 Fallberichte}

Autoren Takacs FZ1 , Hamza $A^{1}$, Yilmaz $U^{2}$, Meyer $S^{3}$, Meyberg-Solomayer $G^{1}$ Institut 1 Klinik für Frauenheilkunde, Geburtshilfe und

Reproduktionsmedizin; 2 Klinik für Diagnostische und Interventionelle

Neuroradiologie; 3 Klinik für Allgemeine Pädiatrie und Neonatologie/ 1 - 3

Universitätsklinikum des Saarlandes

DOI 10.1055/s-0038-1670475

Patienten und Methode Fall 1 Überweisung einer 23-jährigen Erstgravida in der 26+0 SSW wegen zystischer Raumforderung (RF) der hinteren Schädelgrube. Es zeigte sich eine $57 \times 55 \times 61 \mathrm{~mm}$ echoarme, zystische RF mit mehreren echoreichen Anteilen (Koagel) ohne Perfusion, dessen Volumen ca. 47\% des intrazerebralen Volumens betrug. Außer einer milden Ventrikulomegalie und verdrängtem, aber morphologisch normal imponierendem Kleinhirn, fanden sich keine weitere Auffälligkeiten. Der Befund war größenkonstant bis zur 30. SSW, danach zeigte sich eine deutliche, fast komplette Regression und Abnahme der Ventrikulomegalie. Die Entbindung erfolgte per primärer Sectio. Der postnatale Ultraschall und MRT konnten initial noch eine Restthrombose zeigen, im Verlauf mit kompletter Remission. Die neuromotorische Entwicklung war ohne Auffälligkeiten. Eine Makrocephalie wird derzeit mittels seriellen Messungen beobachtet.

Fall 2 Eine weitere 23-jährige Erstgravida wurde in der 24+2 SSW wegen zerebraler Raumforderung zu uns überwiesen. Es fand sich eine echoarme, zystische RF von $34 \times 24 \times 33$ mm, mit einem echoreichen soliden Befund von $18 \times 17 \times 18 \mathrm{~mm}$ (Thrombus). Es konnten keine weitere fetale Auffälligkeiten festgestellt werden. Bis zur 30. SSW war der Befund konstant, später deutliche Regrendienz, so dass dieser in der 35. SSW weder im MRT noch im Ultraschall nachgewiesen werden konnte. Die Entbindung erfolgte per primärer Sectio. Postnatal zeigte die Gerinnungsdiagnostik eine homozygoter 1298 MTHFRMutation. Im MRT konnten Residuen der Thrombose nachgewiesen werden. Der klinische Verlauf war komplett unauffällig.

Schlussfolgerung Eine intrakranielle venöse Thrombose kann vorgeburtlich auftreten und ist durch Sonografie nachweisbar Serielle Ultraschalluntersuchungen und MRT können in der Beurteilung der Prognose helfen. In Zusammenklang mit der Literatur können wir bestätigen, dass eine partielle oder totale Regression bei isolierten Anomalien, sowie fehlender fetaler kardialer Dekompensation auf eine günstige Prognose schließen lassen. Ein Zusammenhang zwischen MTHFR-Mutation und fetaler/neonataler Sinusthrombose konnte bislang nicht festgestellt werden.

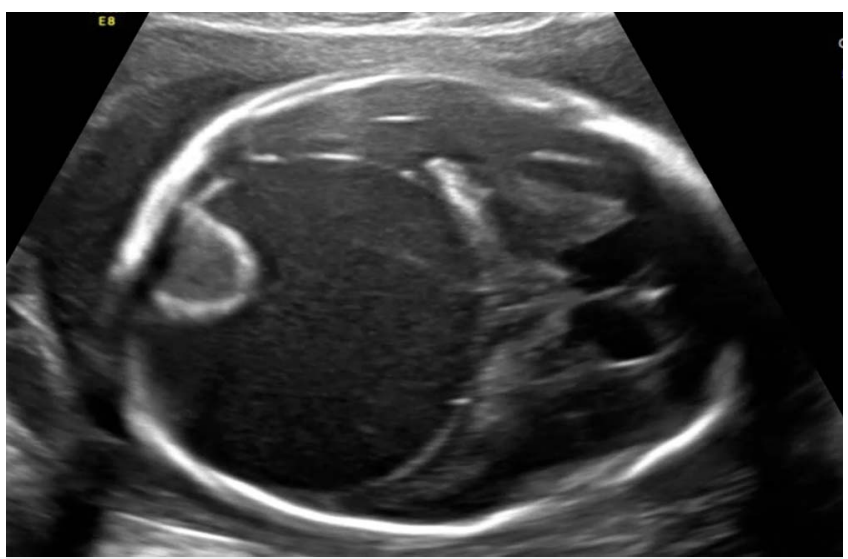

Abb. 1

Wi-Vo 13 Mamma

Fr. 16.11. 13:00 - 14:30 Shanghai 3

\section{13.01 Quantitativer multiparametrischer Ultraschall der Brust führt zu einer erhöhten diagnostischen Genauigkeit}

Autoren Kapetas $\mathrm{P}^{1}$, Clauser $\mathrm{P}^{1}$, Woitek $\mathrm{R}^{1}$, Wengert $\left.\mathrm{G}\right]^{1}$, Lazar $\mathrm{M}^{1}$, Bernathova $\mathrm{M}^{1}$, Pinker $\mathrm{K}^{1}$, Helbich $\mathrm{TH}^{1}$, Baltzer PAT ${ }^{1}$

Institut 1 Universitätsklinik für Radiologie und Nuklearmedizin, Medizinische Universität Wien

DOI 10.1055/s-0038-1670476

Einführung Ultraschall (US) der Brust weist eine hohe Sensitivität aber eine moderate Spezifität auf. Um diese zu erhöhen wurden unterschiedliche funktionelle US-Modalitäten entwickelt, welche zusätzlich ein Quantifizierungsvermögen anbieten. Ziel dieser Studie war die Auswertung des quantitativen multiparametrischen (mp) US der Brust mittels B-Mode, Elastografie, Doppler und kontrastmittelverstärktem US (KMUS) in verschiedenen Kombinationen von 2, 3 oder 4 Parametern für die Unterscheidung von gutartigen und bösartigen Läsionen sowie die Prüfung einer möglichen Variation je nach Erfahrungsniveau des Untersuchers.

Material und Methoden 124 Patientinnen mit jeweils einer sonografisch evidenten, biopsierten Brustläsion wurden im Rahmen dieser prospektiven, durch die lokale Ethikkommission bewilligten Studie mit B-Mode US, Elastografie (Virtual Touch IQ-VTIQ), Doppler und KMUS untersucht. Für jede Modalität wurden unterschiedliche quantitative Parameter erfasst: Scherwellengeschwindigkeit (SWV) für VTIQ, Pulsatilität- (PI) und Widerstandsindex (RI) für Doppler. Für KMUS wurden 11 unterschiedliche Parameter anhand einer de- 
dizierten Software gemessen. 4 Leser (2 erfahrene Brust-Radiologen und 2 Radiologie-Assistenzärzte in Ausbildung) werteten unabhängig voneinander die B-Mode Bilder jeder Läsion aus und vergaben jeweils einen BI-RADS-Score. Diese wurden dann mit den quantitativen Parametern kombiniert. Mittels ROC-Kurvenanalyse und deskriptiver Statistik wurden die unterschiedlichen quantitativen Parameter und die diagnostische Genauigkeit des mpUS bewertet. Histologie diente als Referenzstandard.

Resultate 59 Läsionen waren gutartig und 65 bösartig. SWV, RI und mean transit time waren die Parameter mit der höchsten Diskriminationskraft. MpUS mit drei Parametern (B-Mode, VTIQ und CEUS) wies die höchste diagnostische Genauigkeit auf, unabhängig vom Erfahrungsniveau der Leser (gemittelte AUC 0,812 vs. 0,683 für B-Mode; $p$-Wert 0,0001), während die Kombination aus BMode, VTIQ und Doppler US die zweitbeste war (gemittelte AUC 0,789; pWert 0,0001). Alle anderen Kombinationen (mit 2, 3 oder 4 Parametern) zeigten eine niedrigere AUC. MpUS mit B-Mode, VTIQ und CEUS konnte die Anzahl der falsch-positiven Biopsieempfehlungen signifikant reduzieren $(p<0,0001)$.

Schlussfolgerung Quantitativer mpUS der Brust mit drei Parametern (B-Mode, VTIQ-Elastografie und CEUS) verbessert die diagnostische Genauigkeit von B-Mode US signifikant, unabhängig vom Erfahrungsniveau des Untersuchers.

\section{13.02 Comparison of the diagnostic accuracy between radial and conventional meander-like breast ultrasound in a clinical setting}

Authors Brasier-Lutz $P^{1}$, Jäggi-Wickes $C^{1}$, Schaedelin $S^{2}$, Burian $R^{1}$, Schoenenberger $\mathrm{CA}^{3}$, Zanetti-Dällenbach $\mathrm{R}^{4}$

Institute 1 Gynecology and Obstetrics; 2 Statistics, Clinical Trial Unit; 3 Department of Chemistry, University of Basel; 4 Gynecology/Gynecologic Oncology, Claraspital Basel/1 - 2 University Hospital Basel

DOI 10.1055/s-0038-1670477

Introduction Radial breast ultrasound (rUS) is used in addition to conventional meander-like ultrasound (mUS) to examine intraductal pathologies but is seldom applied in routine breast ultrasound. Here we compare rUS and mUS with respect to the diagnostic accuracy and detection rate of malignancies. Materials and Methods This prospective, single-center, noninferiority study was conducted from August 2011 until August 2014. MUS and rUS were performed in women of an unselected, mixed collective. Consenting women received a bilateral whole rUS and mUS by two independent examiners using the same type of US machine and. RUS was performed with a $92 \mathrm{~mm}$, mUS with a $50 \mathrm{~mm}$ linear probe.

Statistics For both methods the sensitivity, specificity, PPV, NPV and accuracy were presented with $95 \%$ confidence interval. The histologic diagnosis served as gold standard. The difference between benign and malignant lesions was compared using t-test and chi-square tests. Examination time was compared between the mUS and rUS using a Wilcoxon signed rank test with continuity correction.

Results In 2131 eligible combined US examinations, in 148 patients, 168 (7.9\%) suspicious lesions were detected, biopsied and 36 (1.7\%) breast cancers diagnosed. The patients had a mean age of 47.12 years. Women diagnosed with breast cancer (57.82 years) were significantly $(p<0.001)$ older than women with benign lesions ( 44.05 years). Sensitivity was $88.9 \%$ for both methods, and specificity $86.4 \%$ for mUS and $89.4 \%$ for rUS. Accuracy, PPV and NPV were $86.9 \%, 64.0 \%$ and $96.6 \%$ for mUS and $89.3 \%$, $69.6 \%$ and $96.7 \%$ for rUS. Tow malignant breast lesions were characterized as BI-RADS 3 by both methods, corresponding to a false negative rate of 5.6\%. Two breast cancers were missed by one method but correctly identified by the other (cancer missed rate: $5.6 \%$ ). The false positive rate was $13.6 \%(\mathrm{n}: 18)$ for mUS and $10.6 \%$ (n: 14$)$ for rUS. In mUS one ( $0.8 \%)$, and in rUS 3 (2.3\%) benign lesions were not detected but documented by the other method. The mean examination time for rUS $(14.73 \mathrm{~min})$ was significantly $(p<0.01)$ shorter than for mUS (22.54 min).
Conclusion Radial breast ultrasound can safely be performed as an alternative method to conventional meander-like ultrasound in routine whole bilateral breast examination, since cancer detection rate, sensitivity, specificity, accuracy, cancer missed rate, and false negative rate of the two methods are similar. However, rUS took significantly less examination time.

\section{13.03 Comparison of patient perception and ultrasound scanning duration between radial and conventional meander-like breast ultrasound in a clinical setting}

Authors Brasier-Lutz $P^{1}$, Jäggi-Wickes $C^{1}$, Burian $R^{1}$, Schoenenberger $C A^{2}$, Schaedelin $\mathrm{S}^{3}$, Zanetti-Dällenbach $\mathrm{R}^{4}$

Institute 1 Gynecology and Obstetrics; 2 Department of Chemistry, University of Basel; 3 Statistics, Clinical Trial Unit; 4 Gynecology/Gynecologic Oncology, Claraspital Basel/1,3 University Hospital Basel

DOI 10.1055/s-0038-1670478

Introduction Breast ultrasound (US) plays a key role in the examination of breast abnormalities. To this day, US is usually performed in a meander-like manner (mUS). The alternative radial US technique (rUS) scans the breast in a circle around the mammilla and is mainly used for the examination of intraductal pathologies. We report a single-centre, prospective study evaluating patient preference for either US procedure.

Material and Methods MUS and rUS were performed using the same type of US machine, on the same day by two independent examiners. RUS was performed with a $92 \mathrm{~mm}$ and mUS with a $50 \mathrm{~mm}$ linear probe. Consenting patients stated comfort and preference in a visual analogue scale-based (VAS) questionnaire. VAS values of mUS and rUS were compared using Mann-Whitney $U$ test. Duration of rUS and mUS scanning was monitored and mean examination time was compared using Wilcoxon signed rank test. Patient family- and personal history ( $\mathrm{HF}$ and $\mathrm{PH}$ ) were investigated. The association between VAS-ratings and the examination time, the patient's age, the body mass index (BMI), the breast size and the presence or absence of a $\mathrm{FH}$ of ovarian-, endometrial- or breast cancer and of a $\mathrm{PH}$ of previous breast surgeries or biopsies was assessed in a multivariate linear model.

Results RUS was considered significantly more comfortable than mUS $(p<0.001)$. With a mean scanning time of 13 min for m-US and 7 min for $r$ US, rUS was significantly faster than mUS ( $p<0.01) .49 \%$ of the 473 patients filling in the VAS scale-based questionnaire preferred rUS, $44 \%$ had no preference, and only $6 \%$ stated a preference for mUS. The preference for rUS was associated with a significantly higher comfort during rUS $(p<0.001$, MannWhitney $U$ test) and the sensation of reduced pressure $(p<0.001$, Fisher's exact test). Younger women felt significantly less comfortable during mUS $(p=0.038)$ but not during rUS $(p=0.67)$ compared to older women. The Breast size, the women's BMI, a positive $\mathrm{FH}$ or $\mathrm{PH}$ and the duration of either US had no influence on a woman's comfort during either mUS or rUS. Conclusion Our data confirm that patients associate rUS with increased comfort. In particular, pressure reduction seemed to contribute to a preference for rUS. Replacing conventional mUS by rUS may help to improve patient comfort and thereby the compliance.

\section{13.04 Menstrual cycle-related changes in breast density using hand-held Speed-of-Sound Ultrasound}

Authors Ruby $L^{1}$, Sanabria $\left.S\right]^{2}$, Obrist $A^{1}$, Martini $K^{1}$, Forte $S^{3}$, Goksel $O^{2}$, Frauenfelder $T^{1}$, Kubik-Huch $\mathrm{R}^{3}$, Rominger $M B^{1}$

Institute 1 Institute of Diagnostic and Interventional Radiology, University

Hospital Zurich; 2 Computer-assisted Applications in Medicine, ETH Zurich;

3 Department of Radiology, Kantonsspital Baden

DOI 10.1055/s-0038-1670479

Purpose Hand-held speed-of-sound (SoS) ultrasound (US) is an imaging technology for breast density (BD) assessment without ionizing radiation or need for breast compression. The purpose of the study was to investigate changes 
in BD during the menstrual cycle and evaluate inter-breast and -segment changes as well as the reproducibility of the method.

Methods Twenty healthy women with a regular MC were scanned twice with a two-week period in between using a standard US machine and a reflector, which served as a timing reference. Three of these volunteers were scanned twice a week for a period of four weeks. According to their menstrual history, the women were allocated to either the follicular or luteal group. Average SoS $(\mathrm{m} / \mathrm{s})$ and $\Delta \mathrm{SoS}$ (variation SoS; $\mathrm{m} / \mathrm{s}$ ) were assessed in the retromamillary, inner and outer segments of both breasts to determine the breast density. Spearman's rank correlation coefficient rs was calculated to evaluate correlations between breast SoS $/ \Delta$ SoS and patient as well as measured characteristics. Interexaminer and interreader agreements were assessed with the interclass correlation coefficient (ICC). ANOVA and Tukey's test were used to assess differences within the breast as well as changes over the four-week period. Comparisons of the follicular and luteal phases, the acquisitions before and during compression as well as the left and right breast were performed with a student t-test.

Results SoS values showed an inverse correlation with BMI ( $\mathrm{rs}=-0.772$, $p<0.001)$, body weight $(r s=-0.718, p<0.001)$, age $(r s=-0.659, p<0.01)$ and bra cup size ( $r s=-0.494, p<0.05)$. For SoS, the interreader $(I C C=0.990)$ and interexaminer $(I C C=0.979)$ agreements were very high. SoS showed a positive correlation ( $r s=0.956, p<0.001$ ) between the left and right breasts without a statistically significant difference comparing the two acquisitions $(p=0.123)$. For SoS, the comparison before and during compression was not significant $(p=0.639)$ and positively correlated $(r s=0.885, p<0.001)$. There was no statistically significant difference between the SoS of the follicular and luteal phases (ANOVA $p<0.001$ ). SoS of the inner breast segments were significantly lower compared to the middle $(p<0.001)$ and outer segments $(p<0.001)$.

Conclusions This result implies that the diagnostic validity of the SoS-US is not significantly affected by the timing of the scan during the menstrual cycle. Interexaminer- and interreader accordance were high and measurements not influenced by probe pressure.

\section{13.05 Kann der Ultraschall die Sentinelbiopsie ersetzen? - Wie genau ist die Mammasonografie beim Mamma-Ca?}

Autoren Deeken $\mathrm{M}^{1}$, Radosa $\mathrm{J}^{3}$, Papadopoulou I ${ }^{1}$, Weimann $\mathrm{R}^{2}$, Solomayer $\mathrm{EF}^{3}$

Institut 1 Klinik für Frauenheilkunde, Knappschaftsklinikum Saar, Krankenhaus Püttlingen; 2 Institut für Pathologie, Klinikum Saarbrücken;

3 Klinik für Frauenheilkunde, Geburtshilfe und Reproduktionsmedizin,

Universitätsklinikum des Saarlandes

DOI 10.1055/s-0038-1670480

Ziel Wie genau ist die Mammasonografie bezüglich der Karzinomerkennung, der Stadieneinteilung, der Messgenauigkeit, der Diskriminierung benigne/maligne und dem axillären Staging? Kann in bestimmten Konstellationen auf eine axilläre Sentinelnodebiopsie (SLNB) verzichtet werden?

Material und Methode Bei insgesamt 867 Karzinomfällen wurden vom 1.1.2009 bis zum 31.12.2016 prospektiv, von 2007-2009 retrospektiv die sonografischen Messdaten mit dem histologischen Ergebnis verglichen. Es wurden Sensitivität, Tumorgröße und ab 20111080 Stanzbiopsien bezüglich der Verdachtsdiagnose benigne/maligne mit dem histologischen Ergebnis verglichen. Es wurden der axilläre Lymphknotenbefall, die Größe der axillären Lymphknotenmetastase und der Body mass Index (BMI) ermittelt.

Ergebnisse Die Sensitivität bezüglich der Karzinomerkennung lag bei $95 \%$ (686/721). Die Genauigkeit der sonografischen Stadieneinteilung war stadienabhängig: pTis 74\%, pT1 81\%, pT2 65\%, pT3 38\% und pT4 75\%. Im Stadium pTis, PT1 und PT2 lag die Rate insgesamt bei $74 \%$. Die durchschnittliche Messabweichung lag bei 7,1 mm: pT1 $5 \mathrm{~mm}$, pT2 $8 \mathrm{~mm}$ und pT3 $23 \mathrm{~mm}$. Die Dignitätseinschätzung war in $97,5 \%$ korrekt. Das axilläre Staging war bei den 534 pN0-Fällen in 91\% (482/534) richtig, in den Stadien pT1pN0 in 94\%
(222/236) und bei pT2 pN0 in 86\% (167/194). Die falsch negativ-Rate (FNR) lag bei im Stadium PT1 bei $5 \%$ und 14\% bei pT2.

Schlussfolgerung Die Sensitivität und Dignitätseinschätzung der Mammasonografie sind sehr gut. Die Genauigkeit der Messung und Stadieneinteilung nimmt mit zunehmendem Tumorstadium ab. Im Stadium cT1 und cT2 kann bei negativer axillärer Sonografie auf die SLNB verzichtet werden.

Schlüsselwörter: Mammasonografie, axilläres Staging, Tumorgröße, Dignität, Sentinelnodebiopsie. 


\section{Namenverzeichnis / Authors' Index}

A

Abdul-Khaliq H S42

Abele $\mathrm{H} \quad \mathrm{S} 41$

Al Naimi A $\mathbf{S 1 0}$

Altmann J S11, S30

Asbach JC S3

Avni EF S34

Axt-Fliedner R S17, S25, S32, S41

Babel J S35

Bahlmann F S10

Baltzer PAT $\mathrm{S42}$

Banas B S29, S34

Banas MC S29

Becker B S18

Becker WH S8

Beckmann MW S25

Beer S S35

Behnke-Mursch 」 S1

Bennasar M S32

Berberat P S21

Berg C S17, S20

Bernathova M S42

Beyer | $\mathbf{S 1 2}$

Bianchi M S17

Bischoff B $\quad 518$

Bollinger N S4

Borowski M S20

Bosselmann S S11, S32

Brand S S27

Brasier-Lutz P 543

Braun J S11, S16, S17, S25

Brevis F S33

Breysem L S34

Brill FHH S18

Bruckner T S13, S14

Büchel J S19

Bücher K S25

Buechel J S18

Buecker A S20

Bührer C S7

Bürger $T$ S3

Burgmaier K S34

Burian R S43

Burnand I S5

Butenschoen A S18

Butenschön A S19

C

Caca K S36

Caroli A S34

Cattrysse E S15

Cavallaro A S29

Celebi N S22

Cetiner M S34

Clarke A S25

Clauser P S42

$$
\text { D }
$$

da Silva NP S13

Dawczynski K S6, S10

De Maeseneer M S15

Debatin KM S3

Deeken M S44

Degenhardt J S31, S40

Delius M S8, S9

Dobravsky L S26

Docheva D S13

Dohse M S24

Doll J S29

Dombrowski F S7
Eckoldt F S10

Eggensberger T S31, S32

Eixarch E S32

Ellenrieder V S35, S37

Engelter ST S38

Enzensberger C S17, S25, S32, S41

Enzmann T S6

Erard V S5

Eveslage M S25

$$
\mathrm{F}
$$

Falkenberg M S25

Falkenberg MK S20

Fan N S37

Faschingbauer F S25

Feldkamp A S33

Firmann M S5

Fischer C S12, S13, S14, S15, S29

Fischer $S \quad S 41$

Fonseca A S31

Formentini A S35

Forte S S43

Fournelle M S20

Frank M S15

Franke D S34

Frankewycz B S13

Frauendorf V S5, S21

Frauenfelder $\mathrm{T}$ S43

Friebe-Hoffmann U S26

Friedl TWP S26

Fröber R S12

Froehlich C S7

Fröhlich E S22

G

Gabriel H S18

Galle PR S22

Gallinat R S25

Garten L S7

Geipel A S17, S20

Gembicki M S10

Gembruch U S17, S20

Germer U S31, S32

Gießelmann B S9

Gimpel C S34

Glawion EN S36

Goecke T S25

Goertz RS S28, S29

Goette M S32

Goksel O S43

Goncalves M S23

Gonser M S27

Görg C S24, S30

Görtz RS S37

Götte M S17, S25, S41

Gottschalk I S19, S20

Gratacós E S32

Gräter T S1, S35

Graupner O S16, S25, S27, S40, S41

Gress VS S36

Grieme B S37

Griewatz」 S22

Grotz A S36

Gründahl FR S16

Günther M S20

Guntinas-Lichius O S24

Guttmann M S7

Gysel W S6

$\mathrm{H}$

Haddadi-Esfahani A S21

Haffner D S34

Haller B 540

Hammer K S11, S16, S17, S20, S25
Hammersen 」 S25

Hammon M S29

Hamza A S18, S40, S42

Hänle MM S35

Hartge D S10

Hartge DR S40

Hartung EA S34

Hasbargen U S8, S9

Hatzipanagiotou M S31, S32

Haufe T S34

Haug T S13

Hechelhammer L S27

Hecher K S27, S31

Heese S S25

Heinzelmann-Schwarz V S18, S19

Heiser C S23

Helbich TH S42

Helfrich F $\mathrm{S40}$

Hempel M S8

Henne-Bruns D S35

Henningsen L S18

Henrich W S7, S11, S26, S30

Henssler L S13

Herrmann J S41

Herzig N S2

Heubner G S34

Hewener H S20

Hillenbrand A S35

Hofauer B S22, S23

Hoffmann F S37

Hohenfellner $\mathrm{K}$ S33

Holland A S24

Holzmann C S21

Hoopmann M S41

Horn D S11

Hübener C S8, S9

Huber D S31, S32

Huber-Katamay J S9

Hug A S14, S15

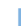

lacoi A $\mathrm{S} 17$

Inwald E S32

Inwald EC S31

Iro H S23

J

Jacobs MJ S38

läggi-Wickes C S43

Janka R S29

Janni W S26

Jarvis JC S24

Juhasz-Boess I S18, S40

Jung EM S13, S29, S34

K

Kagan KO S41

Kalder J S38

Kalle T von S11

Kandel M S15

Kapetas P S42

Karl J S23

Karlas T S35

Kauczor HU S13, S14

Keim V S35

Keschenau PR S38

Kielisch C S28, S29

Kiesl S S15

Kiver V S30

Klee A S27

Kleinhempel A S8

Klieser E S9

Klinger C S36

Klock JH S18

Klockenbusch K S20 
Klockenbusch W S11, S16, S17, S25

Klöckner N S26

Knippel A S26

Knopf A S22, S23

Koch M S23

Kocher S S37

König J S34

Köninger A S25

Korinth D S11

Korten S S24

Köster H S25

Köster HA S16, S20

Kozlowski P S26

Krammer D S14, S29

Krapp M S31, S32

Kratzer W S1, S35, S36

Kreiselmaier $P$ S31

Kreße-Chludek P S26

Krishnabhakdi S S2, S5, S39

Krix M S14

Kubik-Huch R S43

Kunsch S S35, S37

Kunz P S12, S14, S15

Kurkevych A S32

Kuschel B S27, S40

$$
\text { L }
$$

Landvogt C S33

Lazar M S42

Leeuw M S15

Lenchik L S15

Liebau MC S34

Lindner LH S9

Lobmaier SM S27, S32, S40

Lorenz N S34

Lorenz P S2

Lucovnik M S9

Lyrer PA S38

M

Macharzina RR S37

Mahner S S8, S9

Maier R S33

Maisenbacher T S35

Malek N S22

Manegold-Brauer G S18, S19

Mansour N S22, S23

Mantsopoulos K S23

Marquardt JU S22

Marten-Mittag B S21

Martinez JM S32

Martini K S43

Mekahli D S34

Mentzel H] S10

Mertens 」 S5

Messé SR S37

Meyberg-Solomayer B S18, S40

Meyberg-Solomayer G S42

Meyer S S42

Meyer T S7

Micheletti T S32

Mick P S12

Mielke G S11, S32

Miksch M S13

Misikire W S24

Moertl MG S9

Mogghadam A S15

Möhler M S22

Möllers M S11, S16, S17, S20, S25

Moriggl B S24

Morris S S25

Moussa A S3

Mühlbacher T S7

Müller A S20

Müller C S33

Müller G S34

Müller V S25, S41

Mursch K S1
Neef M $\$ 35$

Neesse A S30, S35, S37

Nerlich M S13

Neuenschwander D S6

Neumann FJ 537

Neumann H S22

Neurath MF S28, S29, S37

Nguyen-Tat M S22

Nöldeke S S38, S39

Nuernberg D S5

Nürnberg D S21

Oberhoffer R $\quad$ S40

Obrist A S43

Oelmeier de Murcia K S11, S16, S17, S20, S25

Ong ACM S34

Ortiz JU S27, S32, S40

Ortmann O S31, S32

Ortmann S S21

Ostermayer E S27, S40

Paantjens MA S15

Pahl S S18

Papadopoulos M S31

Papadopoulou I S44

Pape L S34

Parthe H S5

Petzold G S35, S37

Pfeifer C S13

Pfeifer L S28, S29, S37

Pinker K S42

Pitarch D S3

Ponnath M S31

Porsche M S35

Prodan N S41

Proquitte H S10

Proquittè $\mathrm{H}$ S6

Pullankavumkal J S26

Puls W S24

Putz FJ S29, S34

$\mathrm{R}$

Radosa J S18, S40, S44

Rahman KF S22

Raithel M S37

Rastan A S37

Rehn M S7

Rehnitz C $\mathbf{S 2 9}$

Reina H S18, S19

Respondek-Liberska M S32

Reuter B S37

Riecken B $\mathbf{S 3 6}$

Riessen R S22

Ritgen 」 S31, S32, S40

Rizzo G S32

Rjosk-Dendorfer D S9

Rogenhofer N S8

Rolf D S17

Rominger MB S43

Roth A S23

Roth W S22

Ruby L S43

Rüdiger J S4

Ruhland F 57

Rutkowski T S37

$$
\mathrm{s}
$$

Sanabria S] $\$ 43$

Sawatzki M S27

Schad A S22

Schaedelin S $\mathbf{S 4 3}$

Schapher M S23

Schellhaas B \$28, \$29

Schiemann U S5

Schleußner E S6, S10, S12
Schmailzl KJ S5

Schmid P S4

Schmidberger J S1, S35, S36

Schmidmaier G S12, S13, S14, S15, S29

Schmidt R S17

Schmidt T S22

Schmitz R S11, S16, S17, S20, S25

Schneider H S25

Schneider U S6, S10, S12

Schoenenberger CA S43

Schoetzau A S18

Schöll E S4

Schönberger H S19

Schreyer I S12

Schuhkraft J S23

Schulte F S24

Schwarz S S33

Seitz KH S2, S5

Semela D S27

Seuss H S29

Siegmann HJ S26

Sivanathan V S22

Slodki M S32

Solomayer EF S18, S40, S44

Spahn S S10

Speicher D S20

Staub D S2

Steffgen L S34

Stegemann E S3

Steinhard J S16

Steinmann 」 S18

Stepan H S25

Stock K S21

Streifler J S38

Stressig R S31, S32, S40

Strizek B S17, S20

Strobel D S28, S29, S37

Ströder R S18, S40

Stroszczynski C S29

Takacs FZ \$42

Takacs Z S18, S40

Tamm M S4

Tanner M S15

Tavares de Sousa M S27

Tekesin I S16, S17

Thielker \ S24

Thieringer F S22

Thierock M S5

Thomaidis T S22

Tietze U S34

Titieni A S34

Todorov S S5

Torra R S34

Traenka C S38

Trenker C S24, S30

Tretbar S S20

Trinks L S6

政

Uder M S29

Urban P S33

Vach W S37

vd Wense A S8

Vennemann S S21

Verlohren S S11, S26

Vigo $\mathrm{F} \mathrm{S} 18$

Vo Cong M S2

Vogeler $\mathrm{F} \quad \mathrm{S7}$

Voigt C S10, S12

Volk GF S24

W

Wacker-Gussmann A $\mathbf{S 4 0}$

Wagner P S41 
Wakker J S1

Weber J S13

Weber MA S12, S13, S14, S15, S29

Weber S S25

Wegscheider $\mathrm{H} \quad \mathrm{S} 24$

Weichert A S7, S30

Weichert 」 S10, S40

Weimann R S44

Weise A $\quad \mathrm{S} 12$

Weitzel D S33

Wengert G] S42
Wertaschnigg D S9

Weskott HP S30, S39

Wibbeke D S20

Wiegand 」 S35

Wildner D $\$ 37$

Winyard PJD S34

Woeckel A S7

Wohlfart S S25

Woitek R S42

Wolter A S25, S32, S41

Wowra T S3
Y

Yilmaz U S42

Z

Zanetti-Dällenbach R S43

Zeifang F S12, S14

Zeller T S37

Zych-Krekora K S32 